
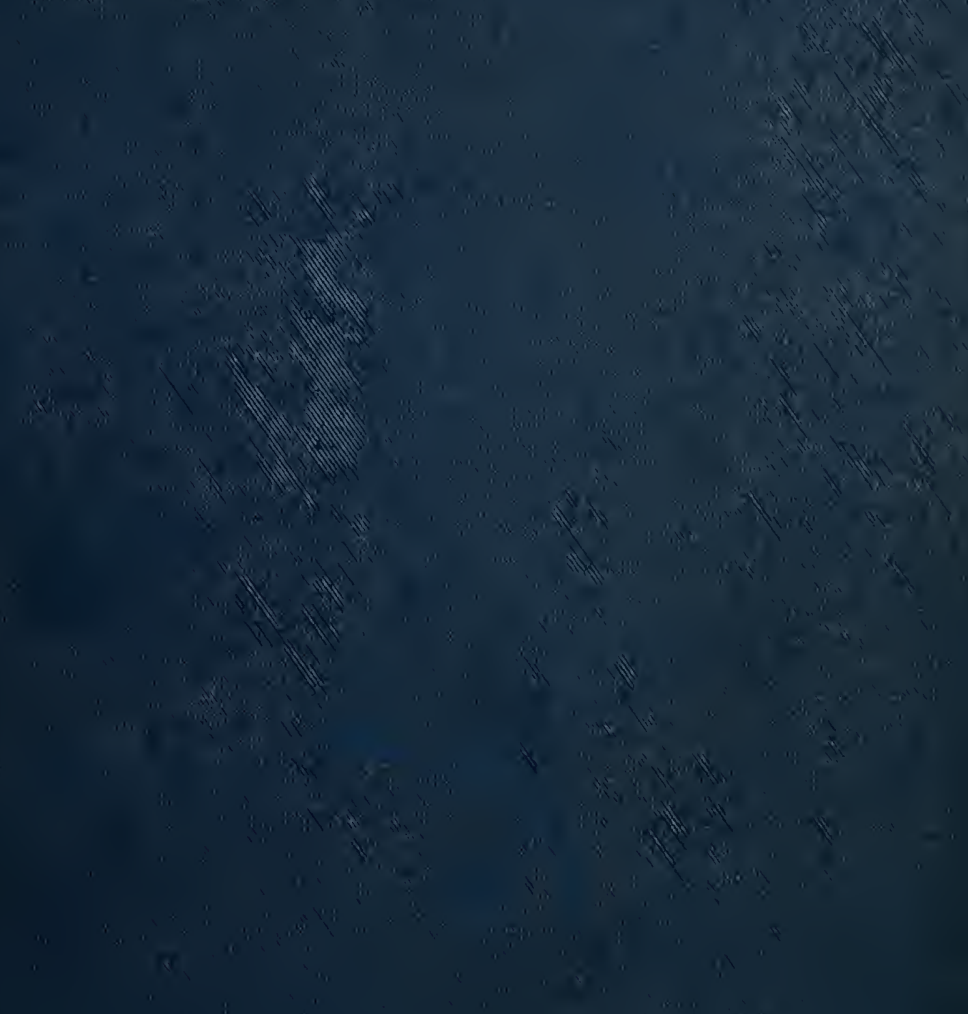

Nive

(1) II) 




\section{HYDROGEN ION CONCENTRATION}





\title{
HYDROGEN ION CONCENTRATION
}

\author{
ITS SIGNIFICANCE \\ IN THE BIOLOGICAL SCIENCES \\ AND METHODS FOR ITS \\ DETERMINATIONS
}

BY

LEONOR MICHAELIS, M.D.

Professor in the University of Berlin, Resident Lecturer in Research Medicine in the Johns Hopkins University

\section{VOLUME I \\ PRINCIPLES OF THE THEORY}

AUTHORIZED TRANSLATION FROM THE SECOND

REVISED AND ENLARGED GERMAN EDITION

\section{BY}

WILliaM A. PERLZWEIG, M.A., PH.D.

Associate in Medicine and Chemist to the

Medical Clinic in the Johns Hopkins

University and Hospital

BALTIMORE

THE WILLIAMS \& WILKINS COMPANY 
Copyright 1926

THE WILLIAMS \& WILKINS COMPANY

Made in United States of America

POBLISHED OCTOBER, 1926

\author{
Composed and Printed at the \\ WAVERLY PRESS \\ FOR
}

The Williams \& Wilkins Compant

BALTimore, MD. 


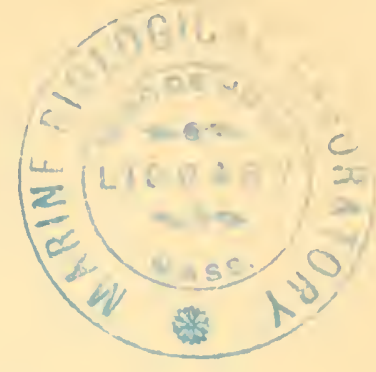

\section{AUTHOR'S PREFACE TO THE SECOND EDITION}

When the first edition of this monograph appeared, the circle of those interested in this field was as yet small, and it gives me pleasure to have contributed to the widening of this circle by means of my little book. Since then, in spite of the external difficulties of the years of war, such an enormous amount of work has been performed in this field, that it would be beyond the power of a single individual to render a complete account of all the details. For better or worse I am obliged to sacrifice completeness of the literature and to present the subject in text-book manner. The scope had to be considerably enlarged, so that the second edition is planned in several volumes of which the present first volume comprises the theoretical physico-chemical principles, while the following volumes will present the methodology and the colloid-chemical, physiological and medical applications. In this way a totally new book will appear which has outgrown the scope of its title, and which may be designated as a "second edition" only for the sake of continuity with its small predecessor.

The subject has been covered in the interval in several textbooks of physiology with various degrees of detail. The most notable monographic account is found in the book by W. M. Clark, The Determination of Hydrogen Ions, Baltimore, 1920. This book naturally has had a significant influence upon my second edition, which influence will be evident in the volume on methods.

The present first volume places the fundamental principles of theory upon a wider basis than was done in the corresponding section of the first edition. The cause for this lies in the first place in the broadened realm of pure physical chemistry, and secondly in the extraordinary growth of the multiplicity of applications of this branch to the other branches of science. Therefore I deemed it important to present to my biological readers the general theoretical principles upon a broad foundation, before the details of their application are dealt with.

LeONor Michaelis.

Berlin, Christmas, 1921. 



\section{AUTHOR'S PREFACE TO THE ENGLISH TRANSLATION}

It was not without hesitation that I yielded to the urging of my American friends and of the publishers in consenting to have this monograph appear in an English translation, for a number of significant advances have been made in this field since the appearance of the original German edition. I finally decided to make at least partial amends by pointing out in a number of addenda within the text the most significant of these recent advances in our science. It is hoped that these will suffice to call the reader's attention to them and to direct him to the original sources for further details and study. The subjects which are especially noted in these addenda are: the recent contributions to the activity theory of ionization by G. N. Lewis, Bjerrum, and Debye; the modification of the theory concerning the dissociation of the ampholytes by Bjerrum; the theory of oxidation-reduction potentials. The latter is sufficiently developed in the new text to give the reader a basis for understanding the application of this theory to the use of the quinhydrone electrode, and to prepare him for the more recent studies in this field, particularly those of W. M. Clark.

I wish to express my gratitude to Dr. W. A. Perlzweig for his painstaking and careful work on this translation.

L. Michaelis.

Baltimore, June 1926. 



\section{TRANSLATOR'S PREFACE}

Professor Michaelis's Wasserstoffionenkonzentration has become well enough known during the past twelve years to my English speaking fellow workers in the biological and related sciences to warrant further introduction or any further statement of the reasons motivating its translation into English, beyond the one of rendering this book more available and useful to them. I am particularly gratified in presenting this monograph with the additions covering the latest developments which were so kindly prepared by Professor Michaelis for this translation. I am greatly indebted to him for this and for his interest and valuable suggestions.

My further debt of gratitude is due to Dr. W. Mansfield Clark of the United States Hygienic Laboratory of Washington for his extremely valuable criticism of the first draft of the manuscript; to my colleague Dr. Arthur Grollman of The Johns Hopkins Medical School for correcting the manuscript; to Dr. Irving H. Page of the Cornell Medical College and to Dr. R. M. Ellsworth of The Johns Hopkins Hospital for their generous help with the proofs; and to my wife, Dr. Olga Marx Perlzweig for her most valuable aid in the furnishing of adequate English equivalents for the more intricate German constructions, and for constant help on all possible occasions. To The Williams \& Wilkins Company I express my appreciation of their generous coöperation in seeing this volume through the press in the most efficient manner.

\section{The Translator.}

Baltimore, June, 1926.

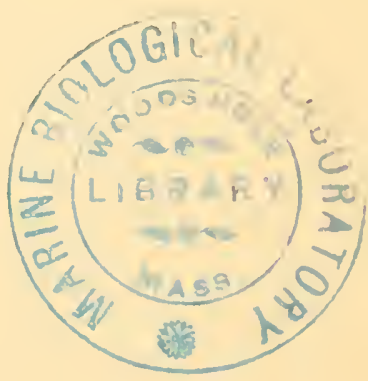





\title{
CONTENTS
}

\section{PART I. THE CHEMICAL EQUILIBRIUM OF THE IONS}

\author{
Chapter I
}

THE LAWS OF ELECTROLYTIC DISSOCIATION

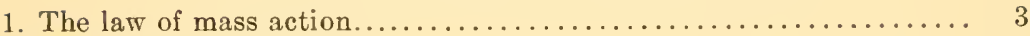

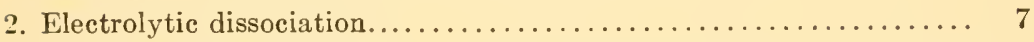

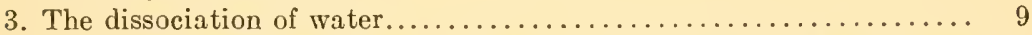

4. The properties of water and its ions. The definitions of acids, bases

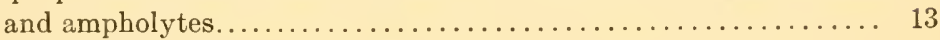

5. The influence of dissolved substances upon the dissociation constant

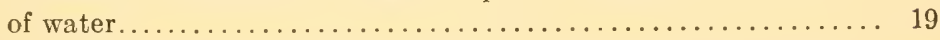

6. The influence of dissolved electrolytes upon the state of dissociation of

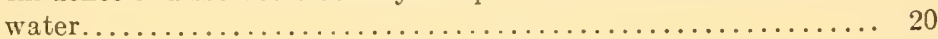

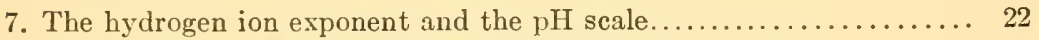

S. The numerical value of the dissociation constant of water......... 24

9. The general application of the mass law to the electrolytic dissociation

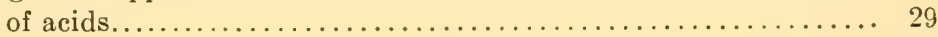

10. True and apparent dissociation constants of acids. Pseudoacids..... 32

11. The dissociation of bases. . . . . . . . . . . . . . . . . . . . . . 37

12. The hydrogen ion concentration in pure acid solutions. . . . . . . 39

13. Mixtures of weak acids and their alkaline salts: Regulators or buffers. 43

14. The two acids problem. . . . . . . . . . . . . . . . . . . . 46

15. The degree of dissociation and the dissociation residue of acids..... 48

16. The dissociation of polybasic acids.................... 55

17. The dissociation of amphoteric electrolytes................. 60

18. The determination of the isoelectric point............... 69

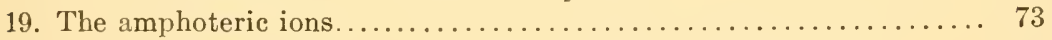

20. The hydrion concentration of a pure solution of ampholyte...... 74

21. The hydrion concentration and the solubility of weak acids...... 77

22. The hydrion concentration and the solubility of difficultly solubleacids. 82

23. The hydrion concentration and the solubility of difficultly soluble

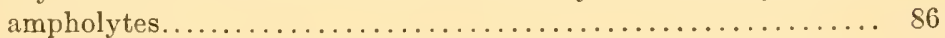

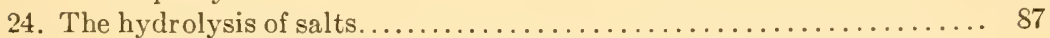

25. Notes on methods for the determination of dissociation constants and of the isoelectric point........................ 91

\section{Chapter II}

THE THEORY OF THE QUANTITATIVE DETERMINATION OF ACIDITY AND ALKALINITY

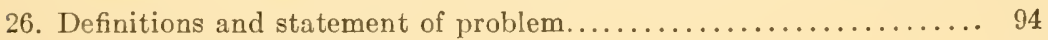

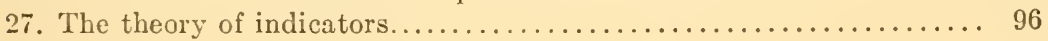


28. The determination of the hydrogen ion concentration, or the estima-

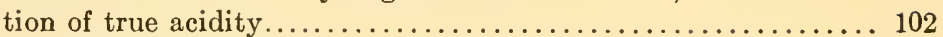

29. The resistance of solutions to change in reaction and buffer value... 104

\section{Chapter III}

THE DISSOCIATION OF STRONG ELECTROLYTES

30. The deviations from the mass law and their significance......... 110

31 . The influence of the ionic charge on the conductivity........... 117

32. The influence of the ionic charge on the lowering of the freezing point. . 119

33. The three deviation coefficients $f_{o}, f_{\mu}$ and $f_{a}$ (according to Bjerrum)... 121

34. Hydration of ions and its signifieance for the activity ........... 123

35 . The theory of activity and the hydrion concentration of buffers.... 127

36. The reduced constants of the physiologically important acids...... 129

37. Another exposition of the effect of strong electrolytes............ 131 Examples of the different methods of representation of the effect of strong electrolytes................................ 133

\section{Chapter IV}

THE STATE OF DISSOCIATION OF ACIDS AND BASES DURING ACTUAL SALT-FORMATION

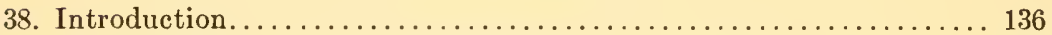

39. The formation of the ionic equilibrium with true salt-formation . . . . 137

40. The behavior of ampholytes in the process of true salt-formation..... 141

41. The influence of salt-formation upon the isoelectric point......... 144

42. An extension of the conception of the reduced dissociation constant... 145

Chapter V

ELECTROLYTIC DISSOCIATION IN NON-ACQUEOUS SOLUTIONS

43. Electrolytic dissociation.............................. 148

PART II. THE IONS, PARTICULARLY THE HYDROGEN IONS, AS SOURCES OF ELECTRIC POTENTIAL DIFFERENCES

44. Introduction.

\section{Chapter VI}

THE ELECTRODE POTENTIALS

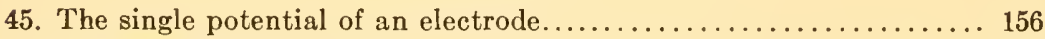

46. The concentration chains............................ 160

47. The numerical evaluation of Nernst's equation............... 161

48. Reversible electrodes for concentration chains................. 163 
49. The theory of gas chains.................................. 164

50. The electromotive series.............................. 167

50a. Oxidation-reduction potentials and the quinhydrone electrode... 169

Chapter ViI

DIFFUSION POTENTIALS (POTENTIAL DIFFERENCES AT LIQUID JUNCTIONS)

51. Diffusion potentials................................ 174

52. The physiological significance of diffusion potentials............ 182

\section{Chapter ViII}

\section{POTENTIALS AT PHASE BOUNDARIES}

53. The origin and calculation of phase boundary potentials......... 183

54. Phase boundary chains.................................... 191

55. The direction of the current in phase boundary chains.......... 195

56. The concentration effect.............................. 196

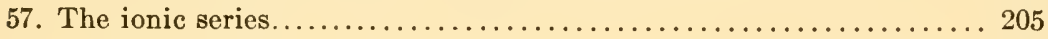

58. The electromotive series of the oils..................... 207

59. The physiological application of the theory of phase boundary

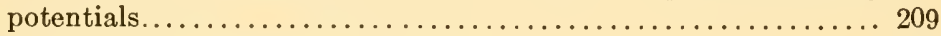

60. Potentials at precipitation membranes .................... 215

61. Polarization phenomena at phase boundaries................ 218

\section{Chapter IX}

\section{MEMBRANE POTENTIALS}

62. The membrane as the cause of a potential................. 220

63. The relation of membrane potentials to phase boundary potentials... 228

\section{Chapter X}

ADSORPTION POTENTIALS AND ELECTROKINETIC PHENOMENA

64. Definition of adsorption................................ 232

65 . The adsorption of electrolytes by charcoal.................. 234

66. The exceptional position of $\mathrm{H}-$ and $\mathrm{OH}$-ions ................ 236

67. Charcoal as an insoluble ampholyte; acidoids, basoids, ampholytoids, saloids....................................... 239

68. The equivalent adsorptions of the ions of a salt by charcoal; the neutralization effect of charcoal.......................... 242

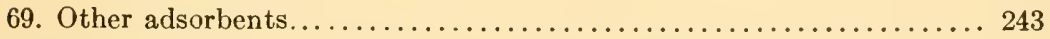

70. The formation of a charge upon phase boundary surfaces due to ad-

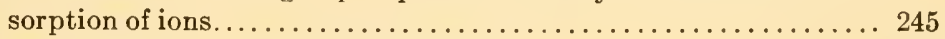

71. The earlier history of electroendosmosis................... 246

72. Helmholtz's theory of electroendosmosis................... 248

73. The theory of electrophoresis......................... 250 
74. The relation of electroendosmosis and electrophoresis to the ionic theory...................................... 254

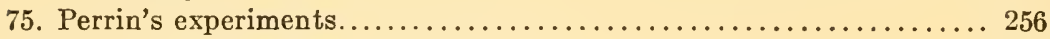

76. The isoelectric point of diaphragms..................... 259

77. Application of the acidoid theory to the sign of charge on diaphragms. . 261

78. A consideration of the other ions......................... 265

79. Summary of the theory of the formation of an electric charge on dia-

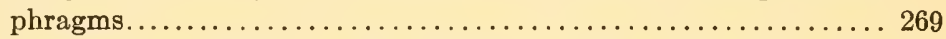

80. Changes in concentration, especially of the H-ions in electroendosmosis...................................... 271

81. The history of the theory of adsorption potentials............... 277

82. Hydrodynamic potentials............................ 279

83. The influence of electrokinetic potentials upon surface tension...... 284

84. A summary of electrokinetic phenomena.................... 284

85. The splitting of phase boundary potentials................. 285

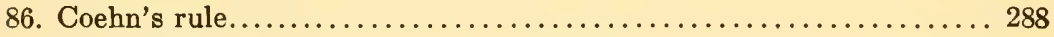

87. The significance of adsorption potentials in physiology and colloidal chemistry ...................................... 290

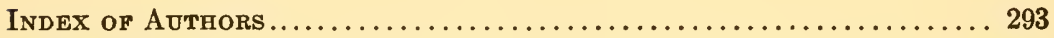

SUbJECT InDEX........................................ 297 


\section{PART I}

The Chemical Equilibrium of the Ions

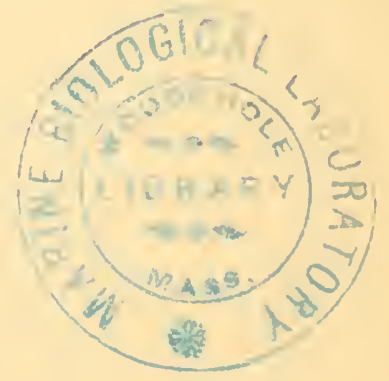





\section{CHAPTER I}

\section{The Laws of Electrolytic Dissociation}

\section{SUMMARY}

The equilibrium of chemical reactions is governed by the law of mass action. Only in the case of dilute solutions can this law be simply and at the same time accurately formulated. This law can also be applied to the dissociation of electrolytes and to the particular case of the dissociation of water. The properties of the ions of water lead to peculiarly specific results. All electrolytes which split off one of the two ions of water change the state of dissociation of water; these are the acids and bases. The concentration of $H$-or $O H$-ions in an aqueous solution determines its acid or alkaline character respectively. The strength of the acids and bases depends closely upon their dissociation constants. The method of calculating the H-ion concentration from the concentration and strength of acids and bases in solution is described, and the development of the theory of regulators or buffers is traced. Through the introduction of special functions of $I$-ion concentration, of the degree of dissociation and of the dissociation residue, the laws of dissociation are reduced to a practically usable form, leading to an explanation of the dissociation of amphoteric electrolytes and to a conception of the isoelectric point. A discussion of the dependence of the solubility of slightly soluble salts upon the Hion concentration and of the hydrolysis of salts is presented.

\section{The law of mass action}

The "law of mass action," as its name implies, is an expression of the effect of the masses of the substances involved in a chemical reaction upon this reaction. Its data relate to the establishment of a definitive state towards which the chemical system is tending, or, to the chemical equilibrium. This state of chemical equilibrium is characterized by the fact that the amounts of the various kinds of participating molecules remain unchanged with the time. It is not, however, a condition of chemical rest, but rather a stationary 
condition in which chemical changes actually occur, but in such manner that at any given instant as much of any given kind of molecule is being produced as is being destroyed. For the maintenance of this stationary condition no external work is required, and conversely no work can be gained from these constantly occurring processes within this stationary state. Furthermore, the algebraic sum of all the work which may be derived from the various component processes of this state, as well as the sum of work expended for these processes, is equal to zero. This differentiates the stationary state of chemical equilibrium from the so called dynamic equilibrium as represented in a living organism. The latter is also a chemical system which may frequently keep its chemical composition unaltered for a long period of time, but for the maintenance of this condition it requires a constant supply of energy.

The apparently resting condition of a living organism does not come within the scope of the above definition of the law of mass action for a closed system. Nevertheless this does not preclude the validity of the application of the mass law to many particular processes occurring in the living organism. Thus processes which progress with a great velocity, such as ionic interreactions, take place also in the living cell in such a manner that a true chemical equilibrium is established. Thus, when free carbonic acid reaches the alkaline blood, a part of it is at once bound, i.e., it produces ions and salts, and exactly the same condition of equilibrium is established, as if the blood were not a component of a living organism. On the other hand the slowly progressing reactions in an organism do not result in the true chemical equilibrium, but at best reach a dynamic equilibrium. For example, the true condition of equilibrium for the combustion of sugar in the blood with the aid of the oxidizing ferments and oxygen is the practically complete destruction of the sugar. This state of true equilibrium is, however, never reached during life, for before it is reached new sugar is brought in from the food or from the glycogen depots, so that the concentration of sugar in the blood is constantly maintained at a fairly fixed level.

In this book we shall deal with ionic reactions only. These occur so rapidly that they always reach true equilibrium. Given two different blood samples containing different concentrations of hydrogen ions, the difference in these two concentrations never signifies that the reaction between the hydrogen ions and the other 
ions has progressed to unequal extents. This reaction occurs with such prodigious velocity that we can have no conception of the intermediate stages; the cause lies in the fact that the two blood samples have actually a different chemical composition. The first chapter of this book deals with the laws of this truc ionic equilibrium, and its basis is, therefore, the law of mass action.

The meaning of the law of mass action is as follows:

1. A simple special case: When any molecular species A decomposes ("dissociates") into two molecular species $B_{1}$ and $B_{2}$, then the equilibrium, i.e., the apparent standstill of the reaction, is reached as soon as

$$
\frac{[\mathrm{A}]}{\left[\mathrm{B}_{1}\right] \cdot\left[\mathrm{B}_{2}\right]}=\mathrm{k}
$$

The brackets denote the molar concentrations of the inclosed substances. The constant $\mathrm{k}$ depends upon the nature of the reaction and the temperature. It is called the affinity constant of the reaction. Its reciprocal value

$$
\frac{\left[B_{1}\right]\left[B_{2}\right]}{[A]}
$$

is called the dissociation constant of the substance $\mathrm{A}$.

2. General case. When 1 molecule $A_{1}+1$ molecule $A_{2}+$ . . are transformed into 1 molecule $B_{1}+1$ molecule $B_{2}+$ . . . equilibrium is reached when

$$
\frac{\left[A_{1}\right] \cdot\left[A_{2}\right] \ldots}{\left[B_{1}\right] \cdot\left[B_{2}\right] \ldots}=k
$$

When two or more of the reacting molecular species are identical, the rule is applied in the same way as above; as for instance in the oxy-liydrogen gas reaction:

1 mol. $\mathrm{H}_{2}+1$ mol. $\mathrm{H}_{2}+1$ mol. $\mathrm{O}_{2} \rightleftharpoons 1$ mol. $\mathrm{H}_{2} \mathrm{O}+1$ mol. $\mathrm{H}_{2} \mathrm{O}$ hydrogen oxygen water vapor

equilibrium is attained when

$$
\frac{\left[\mathrm{H}_{2}\right] \cdot\left[\mathrm{H}_{2}\right] \cdot\left[\mathrm{O}_{2}\right]}{\left[\mathrm{H}_{2} \mathrm{O}\right] \cdot\left[\mathrm{H}_{2} \mathrm{O}\right]}=\mathrm{k}
$$


or abbreviated, the reaction

2 mol. $\mathrm{H}_{2}+1 \mathrm{~mol} . \mathrm{O}_{2} \rightleftharpoons 2 \mathrm{~mol} . \mathrm{H}_{2} \mathrm{O}$

is in equilibrium when

$$
\frac{\left[\mathrm{H}_{2}\right]^{2} \cdot\left[\mathrm{O}_{2}\right]}{\left[\mathrm{H}_{2} \mathrm{O}\right]^{2}}=\mathrm{k}
$$

This law of mass action holds good only under the assumption that all of the interreacting molecular complexes as well as those newly formed coexist in a homogeneous solution (or in gaseous state). If one of the substances involved in a reaction is partially precipitated (removed from solution) because of oversaturation, then the term "concentration" of this substance should comprise only the concentration of its part still remaining in solution.

Should approximate accuracy be accepted as satisfactory, then it could be stated that the law of mass action is confirmed by so large a number of cases that it is to be regarded as a general law also valid for those cases in which the theoretical proof of its correctness could be not obtained. But in attempt at a higher degree of accuracy it must be recognized that the law in the form stated above is only approximately correct. The smaller the concentration of the reacting substances the closer the approximation, and it is closest for ideal gases and for substances reacting in extremely dilute solutions. In fact, the strict thermodynamic test permits the application of the law to these latter extreme cases only. This test leads to a really general law which differs from the above stated law insofar as the term "concentration" is everywhere replaced by the term "pressure." It may either be gas pressure or osmotic pressure. Since, in the state of very great dilution, pressure and concentration are proportional to each other, it follows that under this condition one may simply substitute concentration for pressure. The thermodynamic form of the law is valid for any order of magnitude of pressure. But at high pressures concentration and pressure are no longer proportional. Hence the theoretical explanation of the fact that at the higher concentrations the law of mass action, expressed in terms of concentration, cannot be absolutely correct. In order to render it in an absolutely valid form it would be necessary to multiply every factor involving concentration by an appropriately chosen factor. This factor is generally less than 1. The values so corrected are called active masses. 
The question may be raised: within what limits of concentrations may the mass law be used without corrections? In solutions of non-electrolytes of 0.1 molar concentration the error is insignificant, while it is within allowable limits even in solutions of molar concentration. It has been recently found that these errors or deviations become much greater as soon as one deals with ionized solutions. Since relatively large deviations have been discovered between the ionic equilibrium and the mass law, this fact has to be taken into consideration.

As important as these corrections are whenever the determination of exact numerical figures is involved, nevertheless the fact cannot be over-emphasized that in most cases they are very small. It is, therefore, essentially justifiable to develop first the complicated system of ionic equilibrium on the basis of the simple law of mass action, and then subsequently to explain when and how the necessary corrections are to be applied.

\section{Electrolytic dissociation}

The publication in 1887 by Svante Arrhenius ${ }^{1}$ of the theory of the dissociation of the electrolytes marked the beginning of a new era in physical chemistry. A great mass of facts which had been awaiting a proper explanation became at once comprehensible. The astounding range of importance of this theory is best illustrated by the fact that the number of its consequences is still growing even at present, and all of this part of the book represents but one series of such consequences. The context of the Arrhenius hypothesis is briefly this: The conductivity of an electrolyte in solution does not depend, as it had been hitherto assumed, on its decomposition by the current into ions, but upon the spontaneous splitting of at least part of the electrolyte into ions, when it is brought into solution. These ions may be regarded as independent molecules; their interreactions obey the usual law of mass action, which was first elaborated by Guldberg and Waage and then chiefly by van't Hoff.

Until recently the origin of the opposite free electric charges in dissociated solutions was not clear. The following explanation had been offered²: Space was imagined to be filled with electrically

1 Svante Arrhenius, Zeitschr. f. physikal. Chem. 1, 631 (1887).

${ }^{2}$ W. Nernst, Theoretische Chemie (the older German editions). 
neutral elementary corpuscles of electricity, each of which in turn consisted of one negatively and one positively charged particles. These particles were supposed to serve as an inexhaustible source for electrical charges arising in dissociation. Thus in the dissociation of water an $\mathrm{H}$-atom removes from this reservoir of electricity a positive charge and the OH-residue removes a negative charge. This assumption has become superfluous with the introduction of the Rutherford-Bohr atomic model. The reservoir of these free charges is in the atoms themselves. Each atom consists of a positively charged nucleus, the charge consisting of a definite number of elementary charges. The nucleus is surrounded by negatively charged electrons. In an electrically neutral atom the number of electrons is equal to the number of positive charges upon the nucleus. Thus in the dissociation of an electrically neutral molecule of $\mathrm{H}_{2} \mathrm{O}$ into $\mathrm{H}^{+}$and $\mathrm{OH}^{-}$what happens is that the dissociated $\mathrm{OH}$ radical carries along with it one electron in excess of its electroneutral state, this extra electron having been derived from the $\mathrm{H}$-atom which now becomes the positively charged $\mathrm{H}$-ion, or $\mathrm{H}^{+}$.

The electrons which determine the state of an atom are held by the latter with varying degrees of firmness. If one of the electrons happens to be held in the atom by a loose bond, then it may be easily thrown off, as it were, and, as a result of this loss of a negative charge, the rest of the atom becomes a positively charged ion. This electron is designated as the valence-electron. If two such are present in an atom, it forms bivalent positive ions, etc. On the other hand, there are atoms in which the saturation with their full quota of electrons is not complete, when given in their usual electroneutral state. In these the positively charged atomic nucleus is capable of combining with one or more electrons, and thus to become a uni- or polyvalent negative ion. According to their capacity to give up or to take on electrons the positive and negative elements are differentiated. The Berzelius theory is thus, in a modified form, revived again. When a "positive" (Na) and "negative" (Cl) atom are found together, a competition arises between the two for the labile electron. A number of these pairs of atoms arrange themselves so that the electron leaves the sphere of attraction of the positive atom and is bound by the negative atom; another part becomes so arranged that the valence-electron becomes attached to the negative atom, but without becoming completely de- 
tached from the positive atom. The first arrangement represents the electrolytically dissociated portion, or the ions, while the second represents the undissociated molecules (of $\mathrm{NaCl}$ ). According to the predominance of the first or second mode of atomic arrangement stronger or weaker degrees of electrolytic dissociation are designated.

The condition of dissociation of an electrolyte is not a resting state of equilibrium; for one and the same molecule may in the course of time pass back and forth between the dissociated and undissociated state repeatedly. The "degree of dissociation" is a static quantity only in the sense that it represents the ratio of the dissociated portion to the total amount of electrolyte at a given instant. Since even in the most dilute solutions the number of individual molecules is enormous, the probability that a statistical table composed of figures estimated at different times would yield varying results is very slight. It is in this sense that in a solution of an electrolyte a dynamic equilibrium exists, just as it does in every solution in which an irreversible reaction has run its course to a state of equilibrium.

Radicals, such as $\mathrm{OH}, \mathrm{CN}$, etc., behave in the above respects exactly as the ions of the individual elements do.

Since the atom of hydrogen possesses but a single electron, it follows that the hydrogen ion consists of a bare atomic nucleus carrying a single positive charge.

The theory of the hydrogen-atomic model admits of the possibility of the H-atom taking up a second electron and thus becoming a negative ion. Indeed Moers ${ }^{3}$ (with Nernst) has recently demonstrated that the definite crystalline compound lithium hydride, $\mathrm{LiH}$, on electrolysis when in a molten state produces positive Li-ions and negative $\mathrm{H}$-ions; i.e., the hydrogen is evolved at the anode instead of at the cathode, as is usual. Here it behaves as a halogen, just as it is frequently simply substituted for a halogen in organic compounds. Otherwise it behaves as a metal. In aqueous solution negative $\mathrm{H}$-ions cannot occur.

\section{The dissociation of water}

Water belongs to that group of substances which undergo electrolytic dissociation. The laws of electrolysis have been worked out

${ }^{3}$ W. Nernst, Dtsch. Bunsen-Ges. 1920; K. Moers, Zeitschr. f. anorg. u. allg. Chem. 113, 179 (1920). 
best for aqueous solutions in which water is the solvent medium. Whenever any electrolyte is dissolved in water, the resulting solution contains not only the ions of this electrolyte, but also the ions yielded by the water, namely, $\mathrm{H}^{+}$and $\mathrm{OH}^{-}$ions. While the degree of the dissociation of water is indeed very slight, still, the purest water shows a definite capacity to conduct an electric current. This fact can only be explained on the basis of its electrolytic dissociation, be it ever so slight, if Arrhenius's theory is to be accepted as being correct. F. Kohlrausch and A. Heydeweyller ${ }^{4}$ obtained after repeated distillations of water under rigorous precautions a final value for the conductivity of water, which value could not be diminished or altered by further purifications. In this way they were the first to establish the numerical value for the dissociation of water. Subsequently many other methods were developed for the determination of the same value, which will be described later, and which corroborated Kohlrausch's findings.

The degree of dissociation of water is, as was already stated, very slight, i.e., the $\mathrm{H}^{+}$and $\mathrm{OH}^{-}$ions have a very great tendency to unite and to form $\mathrm{H}_{2} \mathrm{O}$. On the other hand, most electrolytes when dissolved in water dissociate into ions to a much greater extent, and some are even completely dissociated. This unique behavior of water has two noteworthy consequences:

1. If a strong electrolyte, such as $\mathrm{KCl}$ for example, which yields neither $\mathrm{H}^{+}$nor $\mathrm{OH}^{-}$ions, is dissolved in water, then the number of $\mathrm{H}^{+}$and $\mathrm{OH}^{-}$ions yielded by the water itself is so insignificant in comparison with that of the other ions present, that many of the properties of the given solution may be completely accounted for by the ionization of the dissolved electrolyte, as, for example, the conductivity and the depression of the freezing point. The dissociation of the water itself is not, however, influenced by a strong electrolyte dissolved in it.

2. But, if an electrolyte which itself yields either $\mathrm{H}^{+}$or $\mathrm{OH}^{-}$ ions be present in an aqueous solution, then the dissociation of the solvent, water, is fundamentally affected. These special electrolytes have such remarkable properties that they had been placed in a separate class long before the theoretical basis of their peculiar behavior was clearly understood. The electrolytes which give off

"F. Kohlrausch und A. Heydweyller, Zeitschr. f. physikal. Chem. 14, 317 (1894). 
$\mathrm{H}^{+}$ions are those that had been known as "acids" and those giving off $\mathrm{OH}^{-}$ions as "bases," while all the other electrolytes had been designated as "salts."

Thus the $\mathrm{H}^{+}$and $\mathrm{OH}^{-}$ions occupy a peculiar position among the other ions by virtue of their affinity for each other being so great that they are always but very little dissociated, and secondly, because they are the ions of the commonest solvent, water.

The chemical equation representing the dissociation of water is:

$$
\mathrm{H}_{2} \mathrm{O} \rightleftharpoons \mathrm{H}^{+}+\mathrm{OH}^{-}
$$

in which $\mathrm{H}^{+}$stands for the positively charged hydrogen-ion and $\mathrm{OH}^{-}$for the negatively charged hydroxyl-ion.

The other conceivable manner of dissociation of water according to the equation:

$$
\mathrm{H}_{2} \mathrm{O} \rightleftharpoons 2 \mathrm{H}^{+}+\mathrm{O}^{=}
$$

is, quantitatively speaking, so negligible that it is not in any way demonstrable. Since the $\mathrm{H}_{2} \mathrm{O}$ molecule is capable of yielding $\mathrm{H}^{+}$-ions, it may be regarded as that of a dibasic acid dissociating in two successive steps:

$$
\begin{aligned}
& \text { 1. } \mathrm{H}_{2} \mathrm{O} \rightleftharpoons \mathrm{H}^{+}+\mathrm{OH}^{-} \\
& \text {2. } \mathrm{OH}^{-} \rightleftarrows \mathrm{H}^{+}+\mathrm{O}^{=}
\end{aligned}
$$

But as a rule, as far as the present day methods are capable of detecting such changes, the dissociation process of the second step occurs to a very much smaller extent than that of the first step. Furthermore, since in the second step of the dissociation shown above in addition to $\mathrm{H}^{+}$-ions also $\mathrm{OH}^{-}$-ions are produced, water may also be considered as a base. The acidic and basic properties of water are as matter of fact very weak. In this respect water is entirely amphoteric, or, its "reaction" is completely neutral.

In terms of the law of mass action the reaction stated in equation (1) may be expressed as the following equilibrium relationship:

$$
\frac{\left[\mathrm{H}^{+}\right] \times\left[\mathrm{OH}^{-}\right]}{\left[\mathrm{H}_{2} \mathrm{O}\right]}=\mathrm{k}
$$

The brackets in the above equation indicate the concentration of the chemical "species" they inclose, and this concentration is always understood to be in terms of gram-molecules or in mols per 
liter. Since the degree of dissociation of water is extremely small, the concentration of the undissociated $\mathrm{H}_{2} \mathrm{O}$ molecules does not show any measurable decrease, and, therefore, the concentration of the undissociated water is, within the limits of experimental error, equal to the total concentration of the water, which is, of course, a constant quantity. Then by transposing the term $\left[\mathrm{H}_{2} \mathrm{O}\right]$ to the right side in equation (2) and designating $\left[\mathrm{H}_{2} \mathrm{O}\right] \mathrm{k}=\mathrm{k}_{\mathrm{w}}$ the above equilibrium expression becomes:

$$
\left[\mathrm{H}^{+}\right] \cdot\left[\mathrm{OH}^{-}\right]=\mathrm{k}_{\mathrm{w}}
$$

$\mathrm{k}_{\mathrm{w}}$ is the dissociation constant of water, and for every definite temperature it has a definite value.

The active masses indicated in the above formulation of the mass law may be considered as being equal to their respective concentrations only in very dilute solutions and in the ideal gaseous state. The active mass of water indicated in (2) as $\left[\mathrm{H}_{2} \mathrm{O}\right]$ is not easily defined. According to Nernst ${ }^{5}$ its value is to be assumed as one proportional to the vapor pressure of water. Thus, for instance, the values for $\left[\mathrm{H}_{2} \mathrm{O}\right]$ in pure water and in a $1 M$ sugar solution are not quite identical, that of the latter being 2 per cent less. The statement that the active mass of water in different solutions is proportional to their respective vapor pressures is valid only for solutions at the same temperature. The active mass of water in solutions not at the same temperature cannot be assumed as being simply proportional to the vapor pressures.

The dissociation constant of water is then a constant value for any given temperature. It varies with the temperature to a greater extent than the dissociation constants of most electrolytes. This depends on the fact that the value $\left[\mathrm{H}_{2} \mathrm{O}\right]$ varies greatly with the temperature. While it is true, as was stated above, that $\left[\mathrm{H}_{2} \mathrm{O}\right]$ is not to be assumed as being simply proportional to the vapor pressures at varying temperatures, still this value is doubtlessly in some, not as yet clearly understood, manner related to the vapor pressure. The wide variations of $\mathrm{k}_{\mathrm{w}}$ depends not so much upon any significant variations of the value $k$ itself in equation (2) as upon variations in the value of the factor $\left[\mathrm{H}_{2} \mathrm{O}\right]$. A satisfactory explanation of these relations has not as yet been derived either from thermodynamic data or those of the molecular theory. Closely

${ }^{6}$ W. Nernst. Theoretische Chemie, 7th ed. P. 680. 
related to the high degree of dependance of the dissociation constant upon the temperature is the relatively high heat of dissociation of water.

The dissociation constant of water $\mathrm{k}_{\mathrm{w}}$ at $22^{\circ} \mathrm{C}$. amounts to $1 \times$ $10^{-14}$, i.e., one ten-millionth squared. From this it follows that in pure water the $\mathrm{H}^{+}$- and $\mathrm{OH}^{-}$-ions are each present in a concentration of $1 \times 10^{-7}$, or, one ten-millionth gram-ions per liter (one ten-millionth normal in respect to each ion). If this figure seems to be extremely small, it is, nevertheless, a sharply defined value. If it be recalled that one gram-molecule of any substance contains $6.2 \times 10^{23}$ molecules (Loschmidt), then in pure water there must be present $6.2 \times 10^{23} \times 10^{-7}=6.2 \times 10^{16}$ of $\mathrm{H}^{+}$- also of $\mathrm{OH}^{-}$-ions per liter, or $6.2 \times 10^{10}=62$ billion per cubic millimeter.

One liter of water contains $1000 / 18=55.56$ mols of $\mathrm{H}_{2} \mathrm{O}$, or 55.56 $\times 6.2 \times 10^{23}=344 \times 10^{23}$ molecules of $\mathrm{H}_{2} \mathrm{O}$. The same liter on the other hand contains, as seen above, $6.2 \times 10^{16} \mathrm{H}^{+}$-ions. Therefore, out of every 555 million $\mathrm{H}_{2} \mathrm{O}$ molecules one is dissociated.

\section{The properties of water and of its ions. The definitions of acids, bases and ampholytes}

Water occupies a most unique position among liquid compounds. Leaving aside some of its peculiarities, such as its maximal density at $4^{\circ}$, its expansion on freezing, its very great surface tension, etc., only those of its properties will be dealt with here which affect its behavior as a solvent for electrolytes. These special properties are best explained on the basis of its exceptionally large dielectric constant. The significance of this important factor may be conveyed as follows: Two electrically and oppositely charged particles of which one carries a positive charge $e_{1}$ and the other a negative charge $e_{2}$ and separated by a distance $r$ are attracted towards each other with the force equal to $\frac{\mathrm{e}_{1} \times \mathrm{e}_{2}}{\mathrm{r}^{2}}$, when the two are in a vacuum (or with practically the same force in a gaseous medium). When, however, the same two particles are found in a solid or liquid medium, the force of attraction operating between them becomes smaller, and its formulation is now $\frac{e_{1} \times e_{2}}{r^{2} \times D}$. In this expression $D$ represents the dielectric constant of the medium. The greater the value of $\mathrm{D}$ of a medium the smaller is the electrostatic attraction 
between any two oppositely charged particles found in it. Water has almost the largest dielectric constant of all substances as shown in the table 1 (the figures given ${ }^{6}$ are mostly for 17 to $18^{\circ} \mathrm{C}$.).

Only polyhydric alcohols (glycerin) and perhaps the acid amides and the nitriles approach the magnitude of the dielectric constant of water, and formamide and hydrocyanic acid alone surpass it.

Because of this factor all electrostatic forces of attraction are greatly diminished in water and the dissociation of electrolytes dissolved in it is powerfully enhanced. This interdependence between the dielectric constant of the solvent and the degree of

\section{TABLE 1}

Table of dielectric constants

\begin{tabular}{|c|c|}
\hline Hydrogen-gas........... 1.000500 & Aniline....... \\
\hline Air. . . . . . . . . . . 1.000576 & Amyl alcohol.............. 16.0 \\
\hline exane.............. 1.88 & Diamond.............. 16.47 \\
\hline etroleum............. 2.0 & Ammonia.............. 22.0 \\
\hline ood, paper........... 2.0-7.0 & Ethyl alcohol...... \\
\hline enzene.............. 2.26 & Methyl alcohol... \\
\hline live oil............ 3.0 & Nitrobenzene..... \\
\hline Ice $\left(\right.$ at $\left.-18^{\circ}\right) \ldots \ldots \ldots \ldots .16$ & Acetonitrile....... \\
\hline romine............ 3.2 & Glycol.............. 41.2 \\
\hline ctyl alcohol........... 3.4 & Glycerol............... 56 \\
\hline Urea (solid)........... 3.5 & nide $($ fused $) \ldots \ldots \ldots \ldots 59.2$ \\
\hline Ethyl ether........... 4.3 & Glycollic acid nitrile....... 68 \\
\hline hloroform.......... 4.95 & Water................ 81.7 \\
\hline odium chloride (solid).... 6.12 & Formamide............ 94 \\
\hline cetic acid............6 6.3 & Hydrocyanic acid......... 95 \\
\hline
\end{tabular}

dissociation of solute electrolytes is universally applicable, and it was simultaneously discovered in 1893 by Nernst ${ }^{7}$ and J. J. Thomson. ${ }^{8}$

The quantitative relationship between the degree of dissociation of the solute and the dielectric constant D of the solvent was worked out by P. Walden. ${ }^{9}$ He compared the behavior of solutions of a

${ }^{5}$ The figures were taken chiefly from Landolbt-Börnstein, Physikalischchemische Tabellen. Berlin, 1912.

${ }^{7}$ W. Nernst, Göttinger Nachr. No. 12 (1893); Zeitschr. f. physikal. Chem. 13, 531 (1894).

8 J. J. Thomson, Phil. Mag. 36, 320 (1893).

${ }^{9}$ P. Walden, Zeitschr. f. physikal. Chem. 54, 228 (1905), also 94, 263 and 374 (1920). 
certain salt (tetraethyl ammonium iodide) in various solvents having the same degree of dissociation. A simple relationship between $\mathrm{D}$ and $\mathrm{c}$, the corresponding concentration, was obtained

$$
\frac{\mathrm{D}}{\sqrt[3]{\mathrm{c}}}=\text { constant }
$$

or, for an electrolyte possessing the same degree of dissociation in various solvents the corresponding "linear concentration" (i.e., the cube root of the concentration) is proportional to the dielectric constant of the solvent. It can be easily shown that the cube root of the concentration of the solute is inversely proportional to the average distance between any of its adjacent molecules.

The ions of water have also very special properties. According to the Rutherford-Bohr hypothesis of the atomic structure the $\mathrm{H}^{+}$ion consists of one positively charged atomic nucleus unaccompanied by any (negative) electron. It is the smallest mass aggregate bearing a positive charge. The smallest negatively charged body is the electron whose mass is only $1 / 1800$-th of a $\mathrm{H}^{+}$-ion, and an analogously small positively charged mass is unknown. In addition to being the smallest known positively charged particle, the $\mathrm{H}^{+}$-ion has the smallest atomic radius of any atomic structure. Furthermore, since it has no shell of electrons surrounding it, it can reach into closer proximity with negatively charged particles than any other positive ion. Also, since the force of attraction of opposite charges increases as the square of the distance, the $\mathrm{H}^{+}$-ion can be held more firmly by negative ions than any other monovalent positive ion.

The properties of the $\mathrm{OH}^{-}$-ion are not as easily explained on the basis of the atomic model. There is a possibility of presenting this entire question in a way which will obviate the necessity of endowing the $\mathrm{OH}^{-}$-ion with special and extreme properties, and which will permit the unique position of the $\mathrm{H}^{+}$-ion to suffice for the elucidation of the problem.

This particular way may be stated as follows: Of all electrolytes in aqueous solution the acids and bases are the least dissociated. There are a few exceptions to this rule, $\mathrm{HCl}, \mathrm{HNO}_{3}, \mathrm{H}_{2} \mathrm{SO}_{4}, \mathrm{NaOH}$, $\mathrm{KOH}, \mathrm{Ba}(\mathrm{OH})_{2}$ and some others, which are just as strongly dissociated as the salts. But most free acids and bases are weak electrolytes, while, as a general rule, the salts belong to the class of strong 
electrolytes, including also the salts of weak acids and bases, such as ammonium acetate and ammonium carbonate. ${ }^{10}$ In case of the acids the common cause for this behavior is explicable on the basis of the atomic model. The center of the minute $\mathrm{H}^{+}$-ion is able to attain closer proximity than any of the larger ions to the center of electrostatic force of the particles to which it is attracted. The force of attraction increasing greatly with diminishing distance, the $\mathrm{H}^{+}$-ion is held more firmly by the anions than any other cation. This conception does not appear to be capable of ready application to the bases. The $\mathrm{OH}^{-}$-ion is spatially more diffuse than the $\mathrm{H}^{+}$ion. It cannot be in any sense asserted, for instance, that the $\mathrm{OH}^{-}$-ion is held more firmly by cations than the $\mathrm{Cl}^{-}$-ion. The $\mathrm{NH}_{4}^{+}$-ion does not bind the $\mathrm{OH}^{-}$-ion any more firmly than it does the $\mathrm{Cl}^{-}$-ion; i.e., the molecular union $\mathrm{NH}_{4} \mathrm{OH}$ occurs just as sparingly as $\mathrm{NH}_{4} \mathrm{Cl}$ does. Not a single weak base exists in which, analogously to the weak acids, its basic nature is due to its slightly dissociable OH-groups. The countless organic compounds containing $\mathrm{OH}$-groups are, without any exception, ${ }^{11}$ non basic. The $\mathrm{N}$-free organic compounds which exhibit a basic character usually hold, by a double bond, an O-atom capable of forming an oxonium ion, in a way analogous to the formation of $\mathrm{NH}_{4}+$-ions from $\mathrm{NH}_{2}$ groups. The usual explanation of the basic nature of $\mathrm{NH}_{3}$ and of every amine is as follows:

The reaction $\mathrm{NH}_{3}++\mathrm{H}_{2} \mathrm{O} \rightarrow \mathrm{NH}_{4}++\mathrm{OH}^{-}$is assumed. The existence of the molecular entity $\mathrm{NH}_{4} \mathrm{OH}$ is problematic, and there is nothing compelling us to accept its existence; it is only accepted in order to justify the origin of the $\mathrm{NH}_{4}+$ ion. With at least equal justification its origin may be explained by the reaction; $\mathrm{NH}_{3}+$ $\mathrm{H}^{+} \rightarrow \mathrm{NH}_{4}{ }^{+}$, or, stating it in words, $\mathrm{NH}_{3}$ binds an $\mathrm{H}^{+}$-ion and thus becomes an $\mathrm{NH}_{4}+$ ion. Accordingly, a base may be defined as a molecular species, electro-neutral in itself, which on uniting with or binding an $\mathrm{H}^{+}$-ion becomes a positive ion. In complete analogy with the above an acid is defined as a molecular species, electro-neutral in itself, which by splitting off an $H$-ion becomes a negative ion.

The strong alkalies do not apparently conform to this definition of a base. But this view is only superficial. The dissociation of

10 That portion of these salts which is not hydrolyzed is strongly dissociated.

11 Except for the quartenary ammonium bases which are strong electrolytes and which behave in the same way as $\mathrm{NaOH}$, as detailed below. 
$\mathrm{NaOH}$ is usually written as: $\mathrm{NaOH} \rightarrow \mathrm{Na}^{+}+\mathrm{OH}^{-}$, and it seems indeed as if the H-ions play no part in it. But it is to be remembered that the symbol $\mathrm{Na}^{+}$does not represent the actual constitution of this ion. The ions are being constantly hydrated, and while the changing number of molecules of the water of hydration (see below) is not usually included in the formula for the $\mathrm{Na}^{+}$ion, yet a symbol including 1 molecule of $\mathrm{H}_{2} \mathrm{O}$ as $\left(\mathrm{NaH}_{2} \mathrm{O}\right)^{+}$is hardly less justifiable than the symbol $\mathrm{Na}^{+}$. In this method of representation the sodium ion arises from sodium hydroxide by the addition of a $\mathrm{H}^{+}$ion, and not by splitting of an $\mathrm{OH}^{-}$ion.

$$
\mathrm{NaOH}+\mathrm{H}^{+} \rightarrow\left(\mathrm{NaH}_{2} \mathrm{O}\right)^{+}
$$

Or, if it is not desirable to be restricted to the above formulation, the same result can be represented as:

$$
\mathrm{NaOH}+\mathrm{H}^{+} \rightarrow \mathrm{Na}^{+}+\mathrm{H}_{2} \mathrm{O}
$$

That is to say: one molecule of $\mathrm{NaOH}$ binds or otherwise employs one $\mathrm{H}^{+}$ion to form one $\mathrm{Na}^{+}$ion with one molecule of $\mathrm{H}_{2} \mathrm{O}$. In this way the strong bases can be brought within the scope of the above general definition of a base.

If it be desired to include the amphoteric electrolytes in this same general scheme, then they would be defined as molecular species which are capable of both binding and yielding $\mathrm{H}$-ions. If, for example, glycocoll be designated as $\mathrm{H}-\mathrm{R}-\mathrm{NH}_{2}$, its double dissociation will be expressed as:

$$
\begin{aligned}
& \text { 1. } \mathrm{H}-\mathrm{R}-\mathrm{NH}_{2}+\mathrm{H}^{+} \rightarrow\left(\mathrm{HRNH}_{3}\right)^{+} \text {and } \\
& \text { 2. } \mathrm{H}-\mathrm{R}-\mathrm{NH}_{2} \longrightarrow \mathrm{H}^{+}+\left(\mathrm{RNH}_{2}\right)^{-}
\end{aligned}
$$

Hitherto reaction 1 has been usually written as

$$
\mathrm{H}-\mathrm{R}-\mathrm{NH}_{2}+\mathrm{H}_{2} \mathrm{O} \rightarrow \mathrm{H}-\mathrm{R}-\mathrm{NH}_{3} \mathrm{OH} \rightarrow\left(\mathrm{H}-\mathrm{R}-\mathrm{NH}_{3}\right)^{+}+\mathrm{OH}^{-}
$$

but the actuality of the molecular complex $\mathrm{HRNH}_{3} \mathrm{OH}$ is entirely questionable. Therefore, there is the possibility of correlating all aspects of acidity and alkalinity with the $\mathrm{H}^{+}$ion without any reference to the $\mathrm{OH}^{-}$ion. But this is only a possibility and not an absolute necessity. Indeed, there are cases in which it is preferable and easier to explain the formation of an anion by the addition of 
an $\mathrm{OH}^{-}$ion rather than by the loss of an $\mathrm{H}^{+}$ion, as for instance in the formation of the bicarbonate ion:

$$
\mathrm{CO}_{2}+\mathrm{OH}^{-} \rightarrow \mathrm{HCO}_{3}^{-}
$$

As will be shown later, in the discussion of the adsorbability of the ions, the $\mathrm{H}^{+}$ion among the cations and the $\mathrm{OH}^{-}$ion among the anions are also unique because of their extraordinarily great adsorbability. Since the $\mathrm{OH}^{-}$ion occupies just as an exceptional position among the anions as the $\mathrm{H}^{+}$ion among the cations, it is well in discussing the question of acid and base formation not to press the $\mathrm{H}^{+}$ion to the foreground at the expense of the $\mathrm{OH}^{-}$ion. Briefly restated: 'The yielding of an $\mathrm{H}^{+}$ion is completely equivalent to the addition of an $\mathrm{OH}^{-}$ion and reversely. In many cases these two processes are so nearly identical that it is impossible to decide in favor of one or the other mode of reaction.

The following definitions may now be stated: an acid is a molecule, electrically neutral in itself which yields by dissociation $\mathrm{H}^{+}$ ions (or adds to itself $\mathrm{OH}^{-}$ions); a base is a molecule which yields $\mathrm{OH}^{-}$ions (or adds to itself $\mathrm{H}^{+}$ions); an amphoteric electrolyte or an ampholyte is a substance which unites in itself both of these properties.

In these definitions special emphasis is designedly laid upon the words "a molecule electrically neutral in itself." A negatively charged particle may bind $\mathrm{H}^{+}$ions and thereby neutralize its electric charge, e.g., $\mathrm{CH}_{3} \mathrm{COO}^{-}$uniting with $\mathrm{H}^{+}$to form $\mathrm{CH}_{3} \mathrm{COOH}$. Such a molecule which unites with a $\mathrm{H}^{+}$ion and is electrically neutralized is an acid ion or an anion. A molecule, which on uniting with a $\mathrm{H}^{+}$ion acquires a positive charge is a base $\left(\mathrm{NH}_{3}+\mathrm{H}^{+} \rightarrow\right.$ $\mathrm{NH}_{4}{ }^{+}$). A molecule, which binds a $\mathrm{OH}^{-}$ion with the resulting neutralization of its charge is a basic ion or a cation $\mathrm{Na}^{+}+$ $\left.\mathrm{OH}^{-}=\mathrm{NaOH}\right)$. A molecule which on binding $\mathrm{OH}^{-}$ions acquires a negative charge is an acid $\left(\mathrm{CO}_{2}+\mathrm{OH}^{-} \rightarrow \mathrm{HCO}_{3}^{-}\right.$and $\mathrm{SO}_{3}+$ $\mathrm{OH}^{-} \rightarrow \mathrm{HSO}_{4}^{-}$).

A molecule which already has a positive (or negative) charge which on binding an $\mathrm{H}^{+}$ion (or $\mathrm{OH}^{-}$) doubles its charge is a divalent cation (or anion), as illustrated by hydrazine for cations: $\mathrm{NH}_{2} \mathrm{NH}_{2}$ $+\mathrm{H}^{+} \rightarrow \mathrm{NH}_{2} \mathrm{NH}_{3}{ }^{+}$and further $\mathrm{NH}_{2} \mathrm{NH}_{3}{ }^{+}+\mathrm{H}^{+} \rightarrow \mathrm{NH}_{3} \mathrm{NH}_{3}{ }^{++}$, and by carbonic acid for anions: $\mathrm{CO}_{2}+\mathrm{OH}^{-} \rightarrow \mathrm{HCO}_{3}^{-}, \mathrm{HCO}_{3}^{-}+\mathrm{OH}^{-}$ $\rightarrow \mathrm{CO}_{3}=+\mathrm{H}_{2} \mathrm{O}$ (this last hydrated ion reaction can be stated as $\mathrm{HCO}_{3}-\rightarrow \mathrm{CO}_{3}=+\mathrm{H}^{+}$). As will be seen in the chapter on ionic 
adsorption, the above multiplicity of ways of writing reactions is not a futile exercise but quite a useful procedure.

5. The influence of dissolved substances upon the dissociation constant of water

It was stated above that every substance dissolved in water has an influence upon its dissociation constant as a result of changes in the pressure of the water vapor and in the mass of the undissociated water. This influence is however very slight. The vapor pressure of a unimolar solution of a nonelectrolyte (sugar, urea, etc.) is only two per cent less than that of pure water, in solutions of binary electrolytes this effect is approximately doubled. But molar solu-

TABLE 2

\begin{tabular}{c|c|l}
\hline Alcohol & Dielectric constant & $\sqrt{\mathrm{k}_{\mathrm{W}}}$ at $25^{\circ} \mathrm{C}$. \\
\hline per cent & & \\
0 & 84 & $4.6 \times 10^{-7}$ \\
7.4 & 78 & 4.1 \\
24.0 & 67 & 3.4 \\
41.8 & 56 & 2.7 \\
64.8 & 43 & 1.5 \\
86.6 & 32 & 0.73 \\
92.6 & 29 & 0.47 \\
97.4 & 27 & 0.28 \\
99.8 & 26 & 0.30 \\
\hline
\end{tabular}

tions are of such great concentration that they may be left out of consideration in this discussion. And even with such high concentrations the effect upon the value of $k_{w}$ is within the present limits of error involved in the determination of $\mathrm{k}_{\mathrm{w}}$.

Let us next consider the conditions under which a dissolved substance markedly affects the dielectric constant of water. The dielectric constant and $\mathrm{k}_{\mathrm{w}}$ diminish in value in aqueous solutions of alcohol with increasing concentrations of alcohol. But in this case also only the higher concentrations have a marked effect. The figures in table 2 were obtained by Löwenharz ${ }^{12}$ for aqueous alcoholic solutions at $25^{\circ} \mathrm{C}$.

12 Löwenherz, Zeitschr. f. physikal. Chem. 20, 283 (1896). 
(By $\mathrm{k}_{\mathrm{w}}$ the value of $\frac{\left[\mathrm{H}^{+}\right] \times\left[\mathrm{OH}^{-}\right]}{\left[\mathrm{H}_{2} \mathrm{O}\right]}$ is understood, where $\mathrm{H}_{2} \mathrm{O}$ is assumed to be proportional to the partial pressure of the watervapor in an aqueous alcololic solution.) In a unimolar alcoholic solution, which contains 4.6 per cent alcohol, the value of $\mathrm{k}_{\mathrm{w}}$ is not easily distinguishable from that of $\mathrm{k}_{\mathrm{w}}$ for pure water.

For all "watery" phases in living tissues the usual value of $k_{\mathrm{wr}}$ (that of pure water) is assumed. This value, however, may not be applied to such "lipoid" phases as cell membranes, nerve fibers, etc.

It has been stated recently by $\mathrm{R}$. Keller ${ }^{13}$ that colloidal aqueous solutions, especially those of proteins, have a distinctly smaller dissociation constant than pure water. The reason why this highly important problem has not been accurately solved long ago lies in the technical difficulties of determining the dielectric constant in such well conducting fluids as aqueous solutions. If Keller's statement is confirmed, then it will have an important bearing upon the calculation of dissociation constants and of ionic equilibria. The quantitative estimation of these values will not be considered for the time being.

\section{The influence of dissolved electrolytes upon the state of dis- sociation of water}

An electrolyte dissolved in water breaks up more or less completely into its ions. If one of these ionic species is the $\mathrm{H}$-ion, then the concentration of the hydrogen ions of the water is increased a certain number of times. But since equation (3) on page 12 must remain unchanged, it follows that the concentration of $\mathrm{OH}$-ions must decrease the same number of times. If the dissolved electrolyte yields to the aqueous solution $\mathrm{OH}$-ions then the exactly reverse effect occurs. An electrolyte which yields only ions other than $\mathrm{H}$ - or $\mathrm{OH}$-ions has no effect on the dissociation of the water. $\mathrm{H}$-ions are given off by acids and acid salts; $\mathrm{OH}$-ions are produced by bases and basic salts; electrolytes which yield neither of these are the true neutral salts, (as $\mathrm{NaCl}$, for example).

It follows, therefore, that while every aqueous solution must contain $\mathrm{H}^{+}$or $\mathrm{OH}^{-}$ions it may react either acid, neutral or alkaline. A neutral solution is characterized by the fact that it contains equal

${ }^{13}$ R. Keller, Kolloid-Zeitschr. 29, 193 (1921). 
numbers of $\mathrm{H}^{+}$and $\mathrm{OH}^{-}$ions, and at $22^{\circ} \mathrm{C}$. it is $10^{-7}$ normal. An acid solution contains more than $10^{-7} \mathrm{~N} \mathrm{H}^{+}$ions and less than $10^{-7} \mathrm{~N} \mathrm{OH}^{-}$ions, an alkaline solution represents the reverse condition. And yet the strongest of acid solutions is not absolutely free of $\mathrm{OH}^{-}$ions, nor is the strongest alkaline solution entirely free of $\mathrm{H}^{+}$ions. In order to define the state of acidity, neutrality or alkalinity of a fluid it is only necessary to state its $\left[\mathrm{H}^{+}\right]$or $\left[\mathrm{OH}^{-}\right]$. Since the results of such measurements are usually stated in terms of $\left[\mathrm{H}^{+}\right]$, this time-established convention will be retained henceforth

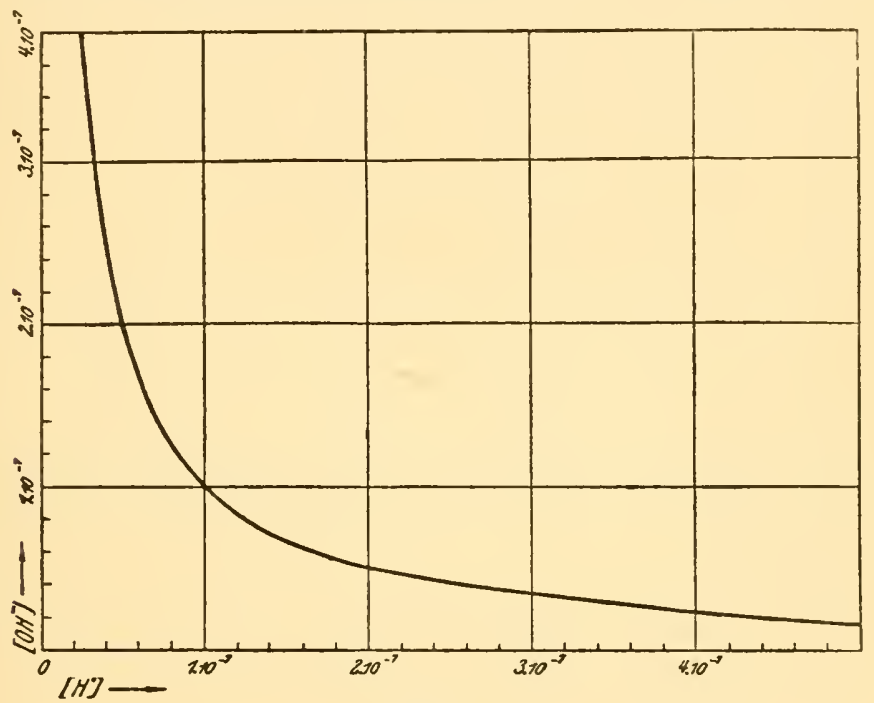

FIG. 1

and the long term "hydrogen ion concentration" will be replaced by the shorter term "Hydrogen number." Therefore, the definition of a

Neutral reaction is: $\left[\mathrm{H}^{+}\right]=10^{-7}$
Acid reaction is : $\left[\mathrm{H}^{+}\right]=>10^{-7}$
Alkaline reaction is : $\left[\mathrm{H}^{+}\right]=<10^{-7}$

The number $10^{-7}$ above is in terms of normality or gram ions per liter.

As stated above the $\left[\mathrm{OH}^{-}\right]$is always dependent upon the $\left[\mathrm{H}^{+}\right]$ in the manner of the following relationship:

$$
\left[\mathrm{OH}^{-}\right]=\frac{\mathrm{k}_{\mathrm{w}}}{\left[\mathrm{H}^{+}\right]}
$$


If the $\left[\mathrm{OH}^{-}\right]$be graphically represented in a rectangular system of coördinates as a function of $\left[\mathrm{H}^{+}\right]$then the hyperbola shown in figure 1 is obtained.

It is of interest to find out at what reaction the sum of $\mathrm{H}^{+}$and $\mathrm{OH}^{-}$ions of a solution is at its minimum. An inspection of the above figure shows that it occurs at the neutral reaction, $\left[\mathrm{H}^{+}\right]=10^{-7}$.

Mathematically this point can be derived in the following way: Let the sought value $\left[\mathrm{H}^{+}\right]+\left[\mathrm{OH}^{-}\right]$be designated as $u$. Then

$$
\begin{aligned}
& \mathrm{u}=\left[\mathrm{H}^{+}\right]+\left[\mathrm{OH}^{-}\right] \\
& \mathrm{u}=\left[\mathrm{H}^{+}\right]+\frac{k_{\mathrm{w}}}{\left[\mathrm{H}^{+}\right]} \\
& \frac{\mathrm{du}}{\mathrm{d}\left[\mathrm{H}^{+}\right]}=1-\frac{k_{\mathrm{w}}}{\left[\mathrm{H}^{+}\right]^{2}}
\end{aligned}
$$

Setting this derivative $=0$, we obtain as the minimal condition

$$
\begin{aligned}
& {\left[\mathrm{H}^{+}\right]=\sqrt{k_{\mathrm{w}}}} \\
& {\left[\mathrm{H}^{+}\right]=\left[\mathrm{OH}^{-}\right]=\sqrt{k_{\mathrm{w}}}}
\end{aligned}
$$

which represents the reaction of neutrality. That this value represents a minimum and not a maximum is shown by the fact that its second derivative

$$
\frac{\mathrm{d}^{2} \mathrm{u}}{\mathrm{d}\left[\mathrm{H}^{+}\right]^{2}}=+2 \frac{\mathrm{k}_{\mathrm{w}}}{\left[\mathrm{H}^{+}\right]^{2}}
$$

must always be positive.

\section{The hydrogen ion exponent and the $\mathrm{pH}$ scale}

The statement $\left[\mathrm{H}^{+}\right]=10^{-7}$ may also be expressed as $\log \left[\mathrm{H}^{+}\right]$ $=-7$, or, $-\log \left[\mathrm{H}^{+}\right]=7$. Sørensen ${ }^{14}$ replaced the term $\log \left[\mathrm{H}^{+}\right]$ by the symbol $\mathrm{pH}^{15}$ The acidic or basic property of a solution

${ }^{14}$ S. P. L. Sörensen, Enzymstudien II. Biochem. Zeitschr. 21, 131 (1909). ${ }^{15}$ Sørensen's original symbol was $\mathrm{p}_{H^{+}}{ }^{\text {; }}$ the present author uses $\mathrm{p}_{\mathrm{h}}$ for $-\log \left[\mathrm{H}^{+}\right]$, and the symbol $\mathrm{h}$ for $\left[\mathrm{H}^{+}\right]$. In order to avoid adding to the already confusing multiplicity of these symbols the form $\mathrm{pH}$ adopted by the American Society of Biological Chemists and the symbol $\left[\mathrm{H}^{+}\right]$now in current use in this country will be adhered to in this translation to designate Sørenson's exponent and the molar concentration of hydrogen ions respectively. The abbreviation "hydrion" for hydrogen ion will also be used.-Translator. 
may thus be just as well defined by stating its $\mathrm{pH}$ as by its $\left[\mathrm{H}^{+}\right]$ value. Quite properly the use of the $\mathrm{pH}$ symbol has become universal. Its advantages are in typographic simplification and also in the fact that the methods for measuring hydrion concentration yields values only in terms of $\log \left[\mathrm{H}^{+}\right]$from which $\left[\mathrm{H}^{+}\right]$must be calculated. This roundabout way is avoided in adopting the $\mathrm{pH}$ units for defining the reaction. It will be furthermore shown that the graphic representation of the effects of $\mathrm{H}^{+}$ions under various conditions in terms of the logarithmic symbol possesses peculiar advantages. The graphic representation of important function yields in this way easily comprehensible symmetric curves, which would not be obtained otherwise. It is best then at once to become accustomed to employ the symbol $\mathrm{pH}$.

TABLE 3

\begin{tabular}{c|l||c|c}
\hline $\mathrm{pH}$ & \multicolumn{1}{|c|}{$\left[\mathrm{H}^{+}\right]$} & $\mathrm{pH}$ & {$\left[\mathrm{H}^{+}\right]$} \\
\cline { 2 - 3 } $\mathrm{n} .00$ & $1.00 \times 10^{-\mathrm{n}}$ & $\mathrm{n} .50$ & $3.16 \times 10^{-\mathrm{n}+1}$ \\
$\mathrm{n} .05$ & $8.91 \times 10^{-\mathrm{n}+1}\left(=0.891 \times 10^{-\mathrm{n}}\right)$ & $\mathrm{n} .55$ & $2.82 \times 10^{-\mathrm{n}+1}$ \\
$\mathrm{n} .10$ & $7.94 \times 10^{-\mathrm{n}+1}$ & $\mathrm{n} .60$ & $2.51 \times 10^{-\mathrm{n}+1}$ \\
$\mathrm{n} .15$ & $7.18 \times 10^{-\mathrm{n}+1}$ & $\mathrm{n} .65$ & $2.24 \times 10^{-\mathrm{n}+1}$ \\
$\mathrm{n} .20$ & $6.31 \times 10^{-\mathrm{n}+1}$ & $\mathrm{n} .70$ & $2.00 \times 10^{-\mathrm{n}+1}$ \\
$\mathrm{n} .25$ & $5.63 \times 10^{-\mathrm{n}+1}$ & $\mathrm{n} .75$ & $1.78 \times 10^{-\mathrm{n}+1}$ \\
$\mathrm{n} .30$ & $5.02 \times 10^{-\mathrm{n}+1}$ & $\mathrm{n} .80$ & $1.59 \times 10^{-\mathrm{n}+1}$ \\
$\mathrm{n} .35$ & $4.47 \times 10^{-\mathrm{n}+1}$ & $\mathrm{n} .85$ & $1.41 \times 10^{-\mathrm{n}+1}$ \\
$\mathrm{n} .40$ & $3.98 \times 10^{-\mathrm{n}+1}$ & $\mathrm{n} .90$ & $1.26 \times 10^{-\mathrm{n}+1}$ \\
$\mathrm{n} .45$ & $3.55 \times 10^{-\mathrm{n}+1}$ & $\mathrm{n} .95$ & $1.12 \times 10^{-\mathrm{n}+1}$ \\
\hline
\end{tabular}

The calculation of $\left[\mathrm{H}^{+}\right]$in terms of $\mathrm{pH}$ may be elucidated by the following example:

1. Let $\left[\mathrm{H}^{+}\right]=1.00 \times 10^{-7} ;$ then $\log \left[\mathrm{H}^{+}\right]=-7$

$$
\text { or } \mathrm{pH}=7
$$

2. Let $\left[\mathrm{H}^{+}\right]=2.00 \times 10^{-7}$; then $\log \left[\mathrm{H}^{+}\right]=0.301-7$

$$
\mathrm{pH}=6.699
$$

Table 3 will be found of use for recalculating $\left[\mathrm{H}^{+}\right]$into terms of $\mathrm{pH} . \mathrm{n}$ in this table is any integral number.

The $\left[\mathrm{H}^{+}\right]$and $\mathrm{pH}$ of some of the more commonly used acids and bases are given below. The dissociation figures of $\mathrm{HCl}$ and $\mathrm{NaOH}$ are calculated from their conductivities (table 4).

Since the dissociation constants of most acids and bases do not 
vary widely with the temperature, the $\mathrm{pH}$ values of acids may be used without great error at any temperature. With bases on the other hand the $\mathrm{pH}$ varies widely, while the $\mathrm{pOH}$ is quite independent of the temperature. The dissociation constant of water changes

TABLE 4

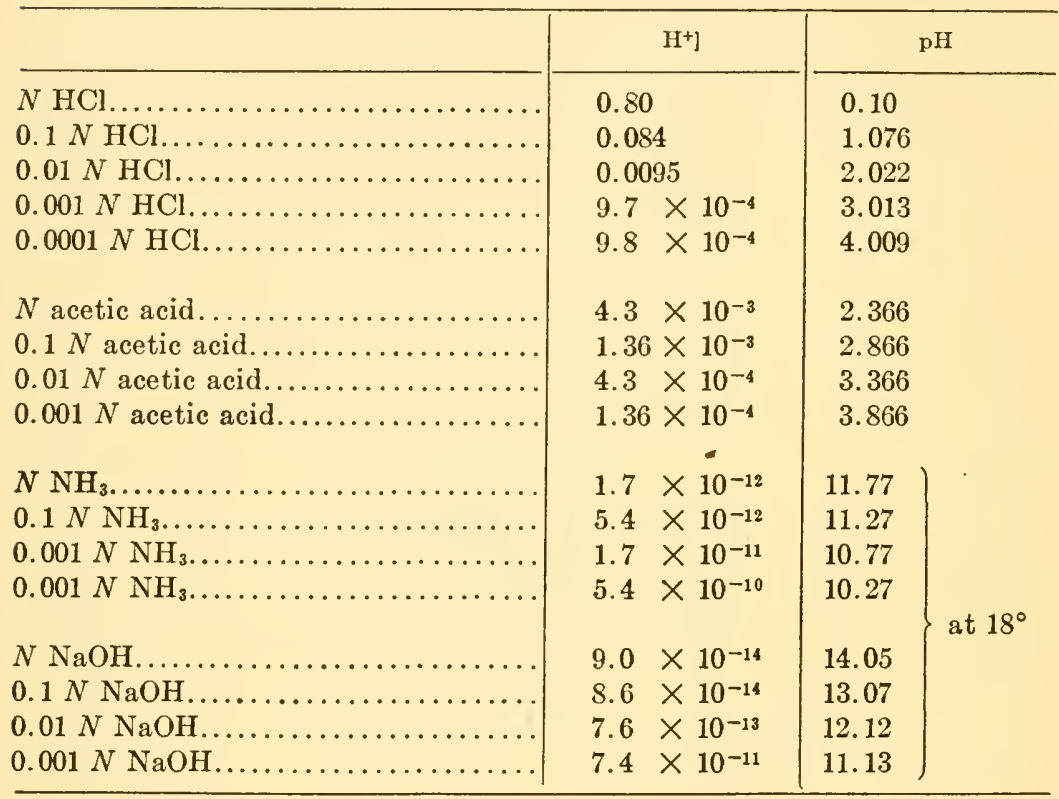

TABLE 5

\begin{tabular}{|c|c|c|c|c|c|}
\hline & & {$\left[\mathrm{H}^{+}\right]$} & $\mathrm{pH}$ & {$\left[\mathrm{OH}^{-}\right]$} & $\mathrm{pOH}$ \\
\hline \multirow{2}{*}{ In $0.01 N \mathrm{HCl}$ at } & $18^{\circ}$ & $9.5 \times 10^{-3}$ & 2.022 & $7.6 \times 10^{-13}$ & 12.12 \\
\hline & $38^{\circ}$ & $9.5 \times 10^{-3}$ & 2.022 & $3.6 \times 10^{-12}$ & 11.44 \\
\hline \multirow{2}{*}{ In $0.1 \mathrm{~N} \mathrm{NH}_{3}$ at } & $18^{\circ}$ & $5.4 \times 10^{-12}$ & 11.27 & $1.7 \times 10^{-12}$ & 11.77 \\
\hline & $38^{\circ}$ & $2.5 \times 10^{-11}$ & 10.60 & $1.7 \times 10^{-12}$ & 11.77 \\
\hline
\end{tabular}

greatly with variations of temperature. On the same basis the $\mathrm{pOH}$ of acids varies considerably with the temperature (table 5).

\section{The numerical value of the dissociation constant of water}

The numerical value of $k_{w}$ is determined by various methods, the principles of which are as follows: 
a. From the conductivity of pure water. F. Kohlrausch and R. Heydeweiller ${ }^{16}$ determined the conductivity of absolutely pure water. Since the conductivities of the $\mathrm{H}$ - and $\mathrm{OH}$-ions were known, the concentration of these ions could be calculated from the observed conductivity of pure water. At $25^{\circ}$ the product $\left[\mathrm{H}^{+}\right] \times$ $\left[\mathrm{OH}^{-}\right]=\mathrm{k}_{\mathrm{w}}=1.1 \times 10^{-14}$.

b. From the catalytic capacity of pure water. At the suggestion of van't Hoff, J. J. A. Wijs ${ }^{17}$ determined the velocity of saponification of methyl acetate by pure water. The $\mathrm{OH}$-ions function as catalyzers and the velocity of the reaction is proportional to their concentration. This observed velocity was compared with that of the same saponification carried out by the use of an alkali solution of known $\left[\mathrm{OH}^{-}\right]$concentration and the $\left[\mathrm{OH}^{-}\right]$of pure water was easily calculated from these data, and furthermore in pure water $\left[\mathrm{H}^{+}\right]=\left[\mathrm{OH}^{-}\right]$. Here it was found that at $25^{\circ}\left[\mathrm{H}^{+}\right] \times\left[\mathrm{OH}^{-}\right]=\mathrm{k}_{\mathrm{w}}=1.44 \times 10^{-14}$.

c. From the degree of hydrolysis of salts. Salts of a weak acid and a strong base in aqueous solution are in part split into free acid

TABLE 6

\begin{tabular}{c|c|c|c|c|c}
\hline & \multicolumn{5}{|c}{ Temperature } \\
\cline { 2 - 3 } & $10^{\circ}$ & $15^{\circ}$ & $25^{\circ}$ & $40^{\circ}$ & $50^{\circ}$ \\
\hline$k_{\mathrm{w}} \ldots \ldots \ldots \ldots \ldots \ldots \ldots$ & 0.31 & 0.46 & 1.05 & 2.94 & $5.17 \times 10^{-14}$ \\
\hline
\end{tabular}

and free base, which in turn yield to the solution by electrolytic dissociation $\mathrm{H}^{+}$and $\mathrm{OH}^{-}$ions. But since the base is stronger, its dissociation predominates, and as a result there is an excess of $\mathrm{OH}^{-}$ions over $\mathrm{H}^{+}$ions. In this case the dissociation constant of the water may be derived, if the dissociation constant of the weak acid is known. This point will be taken up again later. Or the $\left[\mathrm{OH}^{-}\right]$may be determined by a catalytic experiment as above in $(b)$. Arrhenius ${ }^{18}$ obtained from the hydrolysis of sodium acetate at $25^{\circ}$

$$
\mathrm{k}_{\mathrm{w}}=1.21 \times 10^{-14}
$$

From the hydrolysis of other salts several investigators obtained similar figures. Lundén ${ }^{19}$ found from a study of the hydrolysis of salts of a weak acid and a weak base the figures shown in table 6 .

${ }^{16}$ F. Kohlrausch and A. Heydweiller, Zeitschr. f. physikal. Chem. 14, 317 (1894).

13 J. J. A. Wijs, Zeitschr. f. physikal. Chem. 11, 492 and 12, 253 (1893).

${ }^{18}$ Sv. Arrhenius, Zeitschr. f. physikal. Chem. 1, 631 (1887).

${ }^{19}$ H. Lundén, Journ. de Chim. phys. 5, 574 (1907). 
d. The hydrogen ion concentration method. By means of the concentration chains, which are to be described later, the $\left[\mathrm{H}^{+}\right]$ of any solution may be determined. If the $\left[\mathrm{H}^{+}\right]$of a certain $\mathrm{NaOH}$ solution of known $\left[\mathrm{OH}^{-}\right]$is determined by this method then $\mathrm{k}_{\mathrm{w}}$ may be calculated. Wilhelm Ostwald ${ }^{20}$ was the first to call atten-

TABLE 7

\begin{tabular}{|c|c|c|c|}
\hline Temperature & $\mathrm{kw} \times 10^{14}$ & $p \mathrm{k}_{\mathrm{w}}=-\log \mathrm{k}_{\mathrm{w}}$ & $\begin{array}{c}\sqrt{\mathrm{k}_{\mathrm{W}}} \\
\text { i.e., }\left[\mathrm{H}^{+}\right] \text {of pure water } \\
\times 10^{7}\end{array}$ \\
\hline 16 & 0.63 & 14.200 & 0.79 \\
\hline 17 & 0.68 & 14.165 & 0.82 \\
\hline 18 & 0.74 & 14.130 & 0.86 \\
\hline 19 & 0.79 & 14.100 & 0.89 \\
\hline 20 & 0.86 & 14.065 & 0.93 \\
\hline 21 & 0.93 & 14.030 & 0.96 \\
\hline 22 & 1.01 & 13.995 & 1.00 \\
\hline 23 & 1.10 & 13.960 & 1.05 \\
\hline 24 & 1.19 & 13.925 & 1.09 \\
\hline 25 & 1.27 & 13.895 & 1.13 \\
\hline 26 & 1.39 & 13.860 & 1.17 \\
\hline 27 & 1.50 & 13.825 & 1.23 \\
\hline 28 & 1.62 & 13.790 & 1.27 \\
\hline 29 & 1.76 & 13.755 & 1.33 \\
\hline 30 & 1.89 & 13.725 & 1.37 \\
\hline 31 & 2.04 & 13.690 & 1.43 \\
\hline 32 & 2.19 & 13.660 & 1.48 \\
\hline 33 & 2.35 & 13.630 & 1.53 \\
\hline 34 & 2.51 & 13.600 & 1.59 \\
\hline 35 & 2.71 & 13.567 & 1.65 \\
\hline 36 & 2.92 & 13.535 & 1.71 \\
\hline 37 & 3.13 & 13.505 & 1.77 \\
\hline 38 & 3.35 & 13.475 & 1.83 \\
\hline 39 & 3.59 & 13.445 & 1.89 \\
\hline 40 & 3.80 & 13.420 & 1.95 \\
\hline
\end{tabular}

tion to this method; W. Nernst ${ }^{21}$ introduced an important correction into the calculations of the method, and since then it has been used by various authors. Löwenherz ${ }^{22}$ found:

${ }^{20}$ Wi. Ostwald, Zeitschr. f. physikal. Chem. 11, 521 (1893).

${ }^{21}$ W. Nernst, Zeitschr. f. physikal. Chem. 14, 155 (1894).

22 R. Löwenherz, Zeitschr. f. physikal. Chem. 20, 283 (1806). 


\begin{tabular}{c|c}
\hline & \multicolumn{2}{|c}{ Temperature } \\
\hline$k_{\mathrm{w}} \ldots \ldots \ldots \ldots \ldots \ldots \ldots \ldots \ldots \ldots \ldots \ldots$ & $19^{\circ}$ \\
\hline
\end{tabular}

The technic of the concentration chains has been worked out recently in great detail, and it must be considered as the most accurate of the methods. The most reliable measurements were made by S. P. L. Sørensen ${ }^{23}$ and yielded the average figure of

$$
\mathrm{k}_{\mathrm{w}}=0.73 \times 10^{-14}=10^{-14.14} \text { at } 18^{\circ}
$$

Michaelis ${ }^{24}$ using the same method obtained the figures given in table 7. They represent the results of a large number of experiments and of graphic interpolations.

In the same way by determining the $\mathrm{pH}$ of $\mathrm{NaOH}$ solutions, whose pOH was calculated from conductivity data, Lewis, Brighton and Sebastian ${ }^{25}$ found at $25^{\circ} \mathrm{C}$.

$$
\mathrm{k}_{\mathrm{w}}=1.012 \times 10^{-14} ; \mathrm{pk}_{\mathrm{w}}=13.995
$$

Hence for pure water at $25^{\circ}$ we have the practically exact values $\left[\mathrm{H}^{+}\right]=1.00 \times 10^{-7}$, or $\mathrm{pH}=7.00$.

The temperature coefficient of $k_{\mathrm{w}}$ may be calculated from van't Hoff's equation (called the "reaction isochore" by Nernst) which is

$$
\frac{\mathrm{dlnk}_{\mathrm{w}}}{\mathrm{dT}}=-\frac{\mathrm{U}}{\mathrm{RT}^{2}}
$$

By substituting the corresponding numerical values and by converting into common logarithms, the following expression is obtained for $20^{\circ} \mathrm{C}$. :

$$
\frac{\mathrm{dlnk}_{\mathrm{w}}}{\mathrm{dT}}=-\frac{13700}{4.571 \cdot(293)^{2}}
$$

in which we replace $\mathrm{U}$, the heat of the reaction $\mathrm{H}^{+}+\mathrm{OH}^{-}=\mathrm{H}_{2} \mathrm{O}$,

${ }^{23}$ S. P. L. Sørensen, Biochem. Zeitschr. 21, 131 (1909).

24 See first edition (1914) of this book.

25 Lewis, G. N., Brighton, T. B. and Sebastian, R. L., Journ. of the Amer. Chem. Soc. 39, 2245 (1917). 
by the heat of neutralization of $\mathrm{HCl}+\mathrm{NaOH}$ (13700 cal., Berthelot's value). This gives us the value of the coefficient as

$$
\frac{\mathrm{dpk}_{\mathrm{w}}}{\mathrm{dT}}=-0.0349
$$

This value calculated from the author's figures given above for the interval of $16-26^{\circ}$ is $=-0.0340$, which is in good agreement with the theory.

The smaller variations in the newer as compared with the older measurements is explained by a better elimination of errors especially those due to the diffusion potential. In view of the fact that the very numerous studies of Sørensen were all made at the same temperature, and yield a very reliable average value, while those of the author cover a wide range of temperature, the Sørensen figure for $18^{\circ}$ is recommended as the most credible, and the author's figures are to be used for the determination of the temperature coefficient. Sørensen's figure for $18^{\circ}$ is $10^{-14.14}$, which is an average obtained from a number of values varying between $10^{-14.11}$ and $10^{-14.115}$ The author's values for $18^{\circ}$ obtained at different times varied between $10^{-14.09}$ and $10^{-14.13}$. The author's average value obtained in 1913 as given in the above table (7) is $10^{-14.13}$ which agrees well with Sørensen's average.

It will be shown later that all of the $\mathrm{pH}$ measurements made until the present will have to be subjected to a certain correction. The uncertainty is based on the following considerations. The $\left[\mathrm{H}^{+}\right]$of a dilute $\mathrm{NaOH}$ solution is measured by comparison with the $\left[\mathrm{H}^{+}\right]$of a dilute $\mathrm{HCl}$ solution, whose $\left[\mathrm{H}^{+}\right]$is assumed to be known. On the other hand the $\left[\mathrm{OH}^{-}\right]$of the $\mathrm{NaOH}$ solution is assumed to be known. Until recently there was no doubt as to the value of the $\left[\mathrm{H}^{+}\right]$of a $\mathrm{HCl}$ solution of definite concentration, or as to the $\left[\mathrm{OH}^{-}\right]$of a $\mathrm{NaOH}$ solution of definite concentration. These were calculated from the respective degrees of dissociation of the acid and base, which in turn were calculated by comparing the molar conductivities of the solutions at a definite dilution and those at infinite dilutions. As will be seen later these calculations are erroneous. It is possible that the values for $\mathrm{pk}_{\mathrm{w}}$ provisionally recommended above are really smaller (at most 10 per cent smaller). 


\section{The general application of the mass law to the electrolytic dissociation of acids}

It was shown above how electrically neutral molecules like those of water can break up into two oppositely charged ions. The reverse phenomenon by means of which these two ions can combine to form electrically neutral molecules can be shown just as well. It will depend upon the external conditions whether in any given case the reaction will proceed in one or the other direction. A free $\mathrm{H}$-ion and a free $\mathrm{OH}$-ion coming spatially into near proximity of each other as a result of molecular motion will unite to form $\mathrm{H}_{2} \mathrm{O}$. On the other hand the impact of a heterogeneous molecule, occurring in a suitable way, will split off from the $\mathrm{H}_{2} \mathrm{O}$ molecule a $\mathrm{H}$ ion. In general, for every given external condition (especially that of temperature) there will be established a certain statistically considered situation in which the number of ions forming is equal to that of the disappearing ions. But such are exactly the conditions for the application of the law of mass action, and the same symbols as are used for stating the reactions of non-electrolytes may be employed in this case. Thus to the "chemical equation," $1 \mathrm{~mol}$ alcohol $+1 \mathrm{~mol}$ acetic acid $\rightleftharpoons 1 \mathrm{~mol}$ ethyl acetate $+1 \mathrm{~mol} \mathrm{H}_{2} \mathrm{O}$, corresponds the equation:

$$
1 \mathrm{H}^{+}+1 \mathrm{OH}^{-}=1 \mathrm{H}_{2} \mathrm{O}
$$

And the formula of the mass law as used in

$$
\frac{[\mathrm{EtOH}] \times[\mathrm{AcOH}]}{[\mathrm{EtOAc}] \times\left[\mathrm{H}_{2} \mathrm{O}\right]}=\mathrm{k}
$$

corresponds in this case to

$$
\frac{\left[\mathrm{H}^{+}\right] \times\left[\mathrm{OH}^{-}\right]}{\left[\mathrm{H}_{2} \mathrm{O}\right]}=\mathrm{k}, \text { or, }\left[\mathrm{H}^{+}\right] \times\left[\mathrm{OH}^{-}\right]=\mathrm{k}_{\mathrm{w}} \text {. }
$$

This states the law of the electrolytic dissociation of water which has been discussed earlier. The dissociation of an acid dissolved in water will now be considered. If the acid is designated as HS and its component ions as $\mathrm{H}^{+}$and $\mathrm{S}^{-}$, then the mass law can be stated in the form of the following equilibrium equation:

$$
\frac{\left[\mathrm{H}^{+}\right] \times\left[\mathrm{S}^{-}\right]}{[\mathrm{HS}]}=\mathrm{k}
$$


The constant $\mathrm{k}$ is a definite characteristic of every acid. It varies with the temperature although, as a rule, not very greatly. It is the dissociation constant or the affinity constant of the acid. Its magnitude is, as Wilhelm Ostwald pointed out, the only rational measure of the strength of an acid.

In order to avoid misunderstandings the value $\frac{\left[\mathrm{H}^{+}\right]\left[\mathrm{S}^{-}\right]}{[\mathrm{HS}]}$ will be henceforth taken as the "dissociation constant" and its reciprocal $\frac{[\mathrm{HS}]}{\left[\mathrm{H}^{+}\right]\left[\mathrm{S}^{-}\right]}$as the "affinity constant." The dissociation constant is a measure of the force which tends to decompose (ionically) the acid; the affinity constant is the force which tends to reunite its ions. By the terms "force" and "affinity" of a chemical reaction is understood that amount of maximal work $\mathrm{C}$ which may be obtained from the decomposition of one mol of the molecular compound involved, as for instance, in the case when 60 grams of acetic acid decompose into 59 grams of acetate ions +1 gram $\mathrm{H}^{+}$ions. The necessary condition however is that the molecular compound involved be present in one molar concentration, and that a sufficiently large reaction volume be chosen so that the concentration is not measurably affected by the decomposition.

The relation of this amount of work $\mathrm{C}$ to the constant $\mathrm{k}$ is expressed

$$
\mathrm{C}=\mathrm{RT} \cdot \ln \mathrm{k}
$$

where $\mathrm{k}$ has its above given meaning. If the reacting molecular species are in solution in any concentration such as $[\mathrm{AcOH}]$, [Acetate $\left.^{-}\right],\left[\mathrm{H}^{+}\right]$then the maximal work $\mathrm{W}$ obtained from the decomposition of one mole is expressed as:

$$
\mathrm{W}=\mathrm{C}+\mathrm{R} \mathrm{T} \ln \times \frac{[\mathrm{AcOH}]}{\left[\text { Acetate }^{-}\right]\left[\mathrm{H}^{+}\right]}
$$

* See following section.

$\dagger$ The figure given is that found by Michaelis and Garmendia (Biochem. Zeitschr. 67, 431 (1914). Abbott and Bray (J. Amer. Chem. Soc. 31, 760 (1909) had previously found the value $1.95 \times 10^{-7}$. It must be stated at once that in the light of some recent developments (cf. Chapter III) some of the above figures must be revised, as well as the figures in the tables of dissociation constants given on pages 59 and 72 . But none of these newer corrections are large. 
TABLE 8

Table of dissociation constants of some of the weaker acids

The figures given below are compiled from Landolt-Börnstein, PhysikalishChem. Tabellen, 4 Ed. Berlin 1912, also from some more recent data. The observers are Wi. Ostwald, Luther, Bodländer, Rothmund and Drucker, Walker and Cormack, Lundén, A. A. Noyes, Walden, Madsen, H. Euler, Michaelis and Rona.

\begin{tabular}{|c|c|c|c|}
\hline Acid & Temperature & & $\mathrm{k}$ \\
\hline 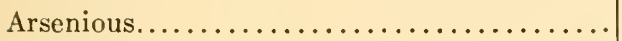 & $25^{\circ}$ & 6 & $\times 10^{-10}$ \\
\hline 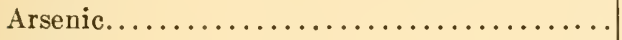 & $25^{\circ}$ & 5 & $\times 10^{-3}$ \\
\hline 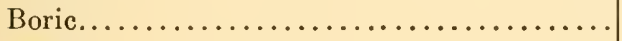 & $15^{\circ}$ & 5.5 & $\times 10^{-10}$ \\
\hline 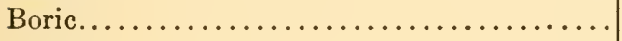 & $25^{\circ}$ & 6.6 & $\times 10^{-10}$ \\
\hline 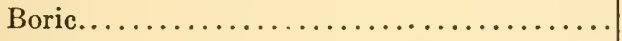 & $25^{\circ}$ & 6.4 & $\times 10^{-10}$ \\
\hline 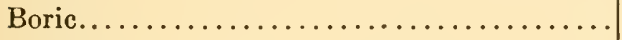 & $40^{\circ}$ & 8.5 & $\times 10^{-10}$ \\
\hline 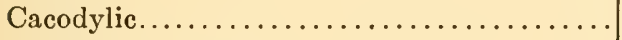 & $25^{\circ}$ & 6.4 & $\times 10^{-7}$ \\
\hline 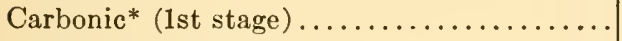 & $18^{\circ}$ & 3.0 & $\times 10^{-7}$ \\
\hline 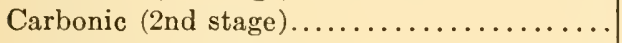 & $25^{\circ}$ & 6 & $\times 10^{-11}$ \\
\hline 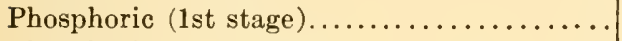 & $25^{\circ}$ & 1 & $\times 10^{-2}$ \\
\hline Phosphoric (2nd stage) $† \ldots \ldots \ldots \ldots \ldots \ldots$ & & 8.8 & $\times 10^{-8}$ \\
\hline Phosphoric (3rd stage)................ & & 3.6 & $\times 10^{-13}$ \\
\hline 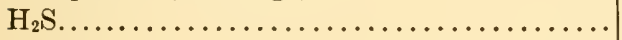 & $18^{\circ}$ & 9.1 & $\times 10^{-8}$ \\
\hline 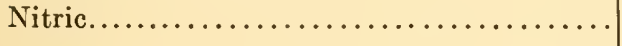 & & 6.0 & $\times 10^{-4}$ \\
\hline Formic.............. & & 2.1 & $\times 10^{-4}$ \\
\hline 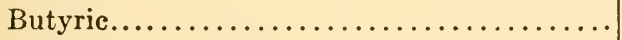 & $18^{\circ}$ & 1.5 & $\times 10^{-5}$ \\
\hline 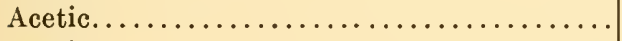 & $10^{\circ}$ & 1.83 & $\times 10^{-5}$ \\
\hline 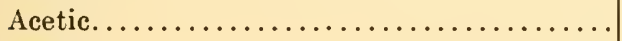 & $18^{\circ}$ & 1.82 & $\times 10^{-5}$ \\
\hline 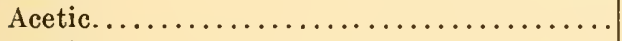 & $25^{\circ}$ & 1.86 & $\times 10^{-5}$ \\
\hline 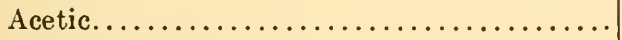 & $40^{\circ}$ & 1.80 & $\times 10^{-6}$ \\
\hline 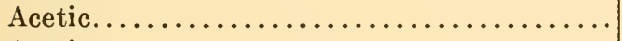 & $50^{\circ}$ & 1.74 & $\times 10^{-5}$ \\
\hline Acetic.................. & $100^{\circ}$ & 1.11 & $\times 10^{-5}$ \\
\hline 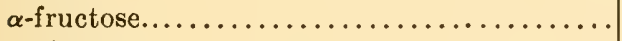 & $18^{\circ}$ & 6.6 & $\times 10^{-13}$ \\
\hline$\alpha$-glucose $\ldots \ldots \ldots \ldots \ldots \ldots \ldots \ldots$ & & 3.6 & $\times 10^{-13}$ \\
\hline 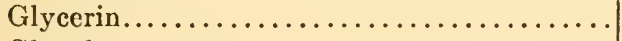 & & 7 & $\times 10^{-15}$ \\
\hline Glycol................. & \multicolumn{3}{|c|}{$<10^{-14}$, extremely small } \\
\hline Lactic.................. & $25^{\circ}$ & 1.38 & $\times 10^{-4}$ \\
\hline$\beta$-oxybutyric................. & $18^{\circ}$ & 3.86 & $\times 10^{-5}$ \\
\hline 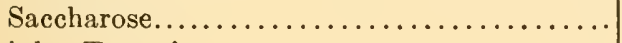 & $18^{\circ}$ & 1.14 & $\times 10^{-13}$ \\
\hline 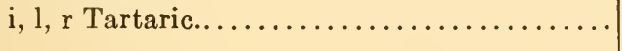 & $25^{\circ}$ & 9.7 & $\times 10^{-4}$ \\
\hline Benzoic.......... & $25^{\circ}$ & 6.0 & $\times 10^{-5}$ \\
\hline 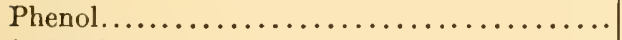 & $25^{\circ}$ & 1.09 & $\times 10^{-10}$ \\
\hline 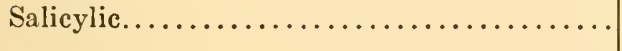 & $20-30^{\circ}$ & 1.05 & $\times 10^{-3}$ \\
\hline Uric. & - & 1.5 & $\times 10^{-6}$ \\
\hline
\end{tabular}

(See footnote at bottom of page $\$ 0$. ) 
In different acids the value of $\mathrm{k}$ varies within wide limits. It could be supposed that every H-atom of a molecule has the tendency to become dissociated into a $\mathrm{H}$-ion. But this tendency depends upon the chemical configuration of the compounds, and under certain conditions it may become extremely small. Thus, for instance, sugar and also alcohol may be conceived of as acids with extraordinarily small dissociation constants, which in the case of sugar can barely be measured, and in the case of alcohol it is so small as to completely escape measurement by any means at our disposal. It may be stated, however, that wherever the methods for the determination of the constant are still sufficient, the constant has a well defined value, in spite of its small order of magnitude.

The upper limit of the dissociation constants cannot be discussed at this point without additional elucidation. The study of the strong acids, such as $\mathrm{HCl}, \mathrm{HBr}, \mathrm{HI}, \mathrm{HNO}_{3}, \mathrm{H}_{3} \mathrm{PO}_{4}$ (the first stage), does not yield uniform values for $\mathrm{k}$, and they will be discussed later. On the other hand, the weaker and the weakest acids follow well in their dissociation the above stated laws. Table 8 gives the dissociation constants of some of the weak acids at $18^{\circ}$. The table also shows the effect of temperature upon $\mathrm{k}$ in the case of a few acids.

\section{True and apparent dissociation constants of acids. Pseudo-acids}

In the case of some acids the free acid is not known to exist in the free state, and only its anhydride is known. Such is the case with $\mathrm{H}_{2} \mathrm{CO}_{3}$ which is not known in its free state, and only its anhydride, $\mathrm{CO}_{2}$, is known. When $\mathrm{CO}_{2}$ is dissolved in water the resulting solution has an acid reaction. From this circumstance it is deduced that $\mathrm{H}^{+}$and $\mathrm{HCO}_{3}{ }^{-}$ions were formed in the solution. The mode of formation of these ions can be explained in two ways: It may be either assumed that the $\mathrm{CO}_{2}$ molecule acquires by addition an $\mathrm{OH}^{-}$ion:

$$
\mathrm{CO}_{2}+\mathrm{H}^{+}+\mathrm{OH}^{-} \rightleftharpoons \mathrm{HCO}_{3}^{-}+\mathrm{H}^{+}
$$

or, since the $\mathrm{H}^{+}$ions play no part in the reaction, as the following:

$$
\mathrm{CO}_{2}+\mathrm{OH}^{-}=\mathrm{HCO}_{3}^{-}
$$


From which follows the equilibrium reaction

$$
\left.\frac{\left[\mathrm{CO}_{2}\right]\left[\mathrm{OH}^{-}\right]}{\left[\mathrm{HCO}_{3}^{-}\right]}=k_{1} !=f \mathrm{HCO}_{3}^{-}\right] \frac{\mathrm{k}_{2}}{\frac{\mathrm{k}_{1}}{\mathrm{k}}} \mathrm{CO}_{2}+C
$$

Substituting for $\left[\mathrm{OH}^{-}\right]$its value $\frac{k_{\mathrm{w}}}{\left[\mathrm{H}^{+}\right]}$as derived from equation (3), page 12 , we obtain

$$
\frac{\left[\mathrm{CO}_{2}\right] \times \mathrm{k}_{\mathrm{w}}}{\left[\mathrm{H}^{+}\right]\left[\mathrm{HCO}_{3}^{-}\right]}=\mathrm{k}_{1}
$$

or

$$
\frac{\left[\mathrm{CO}_{2}\right]}{\left[\mathrm{H}^{+}\right]\left[\mathrm{HCO}_{3}^{-}\right]}=\frac{\mathrm{k}_{1}}{\mathrm{k}_{\mathrm{w}}}=\mathrm{k}_{2}
$$

This equation represents the hypothetical chemical reaction of

$$
\mathrm{CO}_{2} \rightleftharpoons \mathrm{H}^{+}+\mathrm{HCO}_{3}^{-}
$$

and $\mathrm{k}_{2}$ could be used as a true "dissociation constant." But in reality it is only an "apparent" dissociation constant, for the last chemical reaction given above does not occur.

But the formation of ions by carbonic acid in solution can be accounted for in another way. The first step is assumed to be the formation of a hydrate:

$$
\mathrm{CO}_{2}+\mathrm{H}_{2} \mathrm{O} \rightleftharpoons \mathrm{H}_{2} \mathrm{CO}_{3}
$$

and whose equilibrium state is

$$
\frac{\left[\mathrm{CO}_{2}\right]\left[\mathrm{H}_{2} \mathrm{O}\right]}{\left[\mathrm{H}_{2} \mathrm{CO}_{3}\right]}=\mathrm{k}_{3}
$$

By transposing the constant value $\mathrm{H}_{2} \mathrm{O}$ to the right side of the equation and by combining it with $\mathrm{k}_{3}$ to form a new constant we obtain

$$
\frac{\left[\mathrm{CO}_{2}\right]}{\left[\mathrm{H}_{2} \mathrm{CO}_{3}\right]}=\mathrm{k}_{4}
$$

$\mathrm{k}_{4}$ has a very large value, i.e., there is only a small amount of $\mathrm{H}_{2} \mathrm{CO}_{3}$ in the presence of a great excess of $\mathrm{CO}_{2}$. The molecule $\mathrm{H}_{2} \mathrm{CO}_{3}$ dissociates electrolytically:

$$
\mathrm{H}_{2} \mathrm{CO}_{3} \rightleftharpoons \mathrm{H}^{+}+\mathrm{HCO}_{3}^{-}
$$


with the following state of equilibrium

$$
\frac{\left[\mathrm{H}^{+}\right]\left[\mathrm{HCO}_{3}^{-}\right]}{\left[\mathrm{H}_{2} \mathrm{CO}_{3}\right]}=\mathrm{k}_{5}
$$

in which $\mathrm{k}_{5}$ is the true dissociation constant of the real carbonic acid, $\mathrm{H}_{2} \mathrm{CO}_{3}$. But the value of $\left[\mathrm{H}_{2} \mathrm{CO}_{3}\right]$ is not known, and only that of $\left[\mathrm{CO}_{2}\right]$ is known. The latter is in fact the concentration of HERE: total free carbonic acid in solution, for the molecular species $\mathrm{H}_{2} \mathrm{CO}_{3}$ is present in extremely minute concentration as compared with that AN ALGE - of the molecular species $\mathrm{CO}_{2}$ in solution. By substituting for $\mathrm{H}_{2} \mathrm{CO}_{3}$ BRAIC in (4) its value in (3) above, we arrive at

$$
\frac{\left[\mathrm{H}^{+}\right]\left[\mathrm{HCO}_{3}^{-}\right]}{\left[\mathrm{CO}_{2}\right]}=\frac{\mathrm{k}_{3}}{\mathrm{k}_{4}}=\mathrm{k}_{6}
$$

CONSISTEN-This expression is identical (reciprocal) with equation (1). ThereCY feilds fore, $\mathrm{k}_{6}$ is also only an apparent dissociation constant.

A CHARACTER This apparent "dissociation" constant is the only one which one A ATI N can measure. The true dissociation constant of carbonic acid, $\mathrm{H}_{2} \mathrm{CO}_{3}$, remains an unknown quantity. But this apparent constant 0 I APPAR-may be employed as well as a true constant in formal calculations and operations.

According to Thiel and Strohecker ${ }^{26}$ the true dissociation constant of the true $\mathrm{H}_{2} \mathrm{CO}_{3}$ is very large, the minimum probable value being $5 \times 10^{-4}$, according to Strohecker ${ }^{27}$ it is $4.4 \times 10^{-4}$, which is a much greater value than those of acetic and formic acids. This is quite plausible, since $\mathrm{H}_{2} \mathrm{CO}_{3}$ is in fact oxyformic acid. An idea of the relative strength of true carbonic acid may be gathered from the following simple experiment: A few drops of neutral red are added to some of a $0.1 \mathrm{~N} \mathrm{NaHCO}_{3}$ solution. The indicator shows a yellow color in the alkaline solution. Just enough of $0.1 \mathrm{~N} \mathrm{HCl}$ is added to change the color of the solution to red, thus indicating an acid reaction. On waiting a few seconds it is observed that the color changes back to yellow. Upon a further addition of $\mathrm{HCl}$ the color becomes red again and then slowly returns to a yellower stage. The reason for this phenomenon lies not in the gradual escape of gaseous $\mathrm{CO}_{2}$ but in the

26 Thiel, A. and Strohecker, R., Ber. d. Dtsch. Chem. Ges. 47, 945 (1914).

27 Strohecker, Zeitschr. f. Unters. d. Nahr-u. Genussm. 31, 121 (1916). 
liberation by the $\mathrm{HCl}$ from the bicarbonate of the true and strong $\mathrm{H}_{2} \mathrm{CO}_{3}$, which then gradually decomposes into $\mathrm{CO}_{2}$ and $\mathrm{H}_{2} \mathrm{O}$. This experiment is made possible by the relatively slight velocity of the reaction

$$
\mathrm{H}_{2} \mathrm{CO}_{3} \rightarrow \mathrm{H}_{2} \mathrm{O}+\mathrm{CO}_{2}
$$

This case of an "apparent dissociation constant" is by no means limited to carbonic acid alone. It must be remembered that it is in reality quite impossible to distinguish in a given case a true from an apparent dissociation constant. This point is so important that it will be amplified by some examples. Glucose is an extraordinarily weak acid. In attempting to picture the chemical constitution of glucose and of its ions we face the possibility of representing the constitution of this sugar in a number of various ways. We may regard it, according to E. Fischer's original structural formula, as an aldehyde, or, according to Tollens, as a cyclic compound with its ring closed by means of one O-atom. The latter may exist in two stereo-isomeric forms as the $\alpha$ - and $\beta$-glucose. Recently even more isomers of the cyclic kind were found. All of these forms are actual and coexist in a state of equilibrium. Furthermore, another configuration is possible, the enolic form of Wahl and Neuberg which is derived from Fischer's aldehyde form. The formulas below show this derivation: I is the aldehyde which in II forms a hydrate and, as is shown in II, the hydrate splits off a molecule of $\mathrm{H}_{2} \mathrm{O}$ again to form the enol form shown in III.

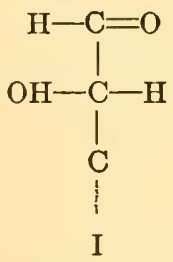

Aldehyde

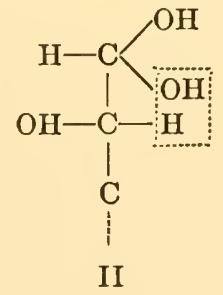

Aldehyde hydrate

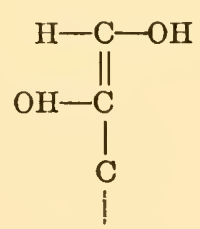

III

Enol

This enolic form is common to glucose, mannose and fructose. Thus, when this enol adds a molecule of $\mathrm{H}_{2} \mathrm{O}$ and passes to the aldehyde again, the resulting sugar of this process may be either glucose, mannose or fructose, and in fact the velocity of this process 
is inversely proportional to the hydrion concentration..$^{28}$ Since the concentration of the sugar ions must be also inversely proportional to the $\left[\mathrm{H}^{+}\right]$, it may then be surmised that the transformation of the sugar must occur through its ions. Furthermore, since, as was shown above, this transformation must occur by means of the enolic form, it follows that the ions must be in an enolic form, and that there cannot be any enolic sugar present in this process beside the ions. In other words: Sugar is a dissociable acid only in its enolic form, and in this form it is a quite strong acid. This is entirely plausible, since all enols are strong acids, while their corresponding aldehydes or ketones are not. And the sugar is a weak acid only because its only modification which possesses acid properties is present in such small amounts in comparison with its other chemical forms.

Many analogous cases are met with among the indicators. Phenolphthalein has an apparently very low dissociation constant. In the free state it has the constitution shown in I below. This form is tautomeric with the form shown in II.
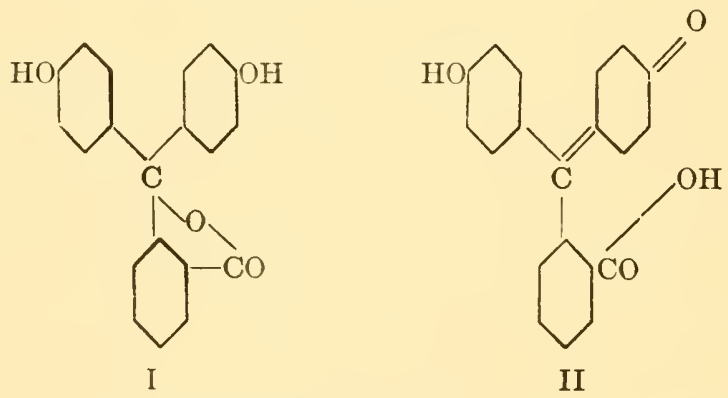

The form I is colorless, while the tautomer II because of its quinonoid double bond is colored, and because of its carboxyl group strongly acidic. The latter form cannot exist in appreciable amounts together with the colorless isomer and only its ions may so occur, and the constitution of these ions may only be the one shown by formula II. Thus the colorless phenolphthalein I is considered as a very weak acid whose ions are colored red, only because in its solutions modification II, which is a strong acid, is present in minute concentrations. All color changes of the indicators depend upon

${ }^{28}$ L. Michaelis and P. Rona, Biochem. Zeitschr. 47, 447 (1912). 
the differences between the constitution of their ions and the undissociated molecules, and the dissociation constants of all indicators are of the "apparent" type.

Those molecular species which are not acid by virtue of their constitution, but which may be transformed by tautomeric change into acids were designated by Hantzsch ${ }^{28 a}$ as pseudo-acids.

The proof for this conception can be demonstrated in a way analogous to that used above for the case of carbonic acid. When acid is added to a red alkaline solution of phenolphthalein the first change ensuing is to the red undissociated acid of form II, and then secondarily the change to the colorless form I. With phenolphthalein, however, these steps occur so rapidly that they cannot be distinguished. But Hantzsch succeeded in demonstrating the phenomenon with suitable dyes, such as crystal violet and many others. When alkali is added to a solution of one of these dyes and the conductivity is determined at the same time, it is found that it decreases with time, showing the disappearance in the solution of a strong electrolyte.

A simple experiment with a slowly changing indicator may be performed as follows. To 100 cc. of a weakly alkaline solution, such as conductivity water or a buffer solution of phosphates, is added one cc. of acid fuchsin or of water blue (1:1000). The solution becomes gradually paler until the color reaches a definite intensity depending upon the $\mathrm{pH}$ of the solution, at which intensity it becomes stationary. At room temperature this process may take one half to one hour, while at $50^{\circ}$ but a few minutes. Acid fuchsin and water blue are indicators in which the two steps described above are distinctly separated. The establishment of ionic equilibrium, like all other ionic reactions, must occur with great rapidity, whereas the tautomeric transformation of the colored form of an indicator into the colorless is a slower process.

\section{The dissociation of bases}

The same holds true for the dissociation of bases as for acids, if $\left[\mathrm{OH}^{-}\right]$is substituted wherever $\left[\mathrm{H}^{+}\right]$occurs. If the molecule of a base be designated as $\mathrm{BOH}$, then its dissociation occurs as

$$
\mathrm{BOH} \rightleftharpoons \mathrm{B}^{+}+\mathrm{OH}^{-}
$$

28 See footnote, p. 97. 
and the dissociation constant is

$$
\mathrm{k}=\frac{\left[\mathrm{B}^{+}\right]\left[\mathrm{OH}^{-}\right]}{[\mathrm{BOH}]}
$$

The principles of the apparent dissociation constants apply also in this case as well as for the acids. Here also it is necessary to differentiate between strong and weak bases. The strong bases are the caustic alkalis, such as $\mathrm{NaOH}, \mathrm{KOH}$. They are ionized to a great extent, and the exact determination of their dissociation relations is fraught with the same difficulties as in the case of the strong

TABLE 9

Dissociation constants of some weak bases

\begin{tabular}{|c|c|c|}
\hline & Temperature & $\mathbf{k}$ \\
\hline Ammonia.. & $10^{\circ}$ & $1.63 \times 10^{-5}$ \\
\hline Ammonia....... & $18^{\circ}$ & $1.75 \times 10^{-5}$ \\
\hline Ammonia.... & $25^{\circ}$ & $1.87 \times 10^{-5}$ \\
\hline Ammonia........................... & $40^{\circ}$ & $1.98 \times 10^{-5}$ \\
\hline Ammonia......................... & $50^{\circ}$ & $1.96 \times 10^{-5}$ \\
\hline Ammonia......................... & $100^{\circ}$ & $1.35 \times 10^{-5}$ \\
\hline Ethylamine $\ldots \ldots \ldots \ldots \ldots \ldots \ldots \ldots \ldots$ & $25^{\circ}$ & $5.6 \times 10^{-4}$ \\
\hline Diethylamine..$\ldots \ldots \ldots \ldots \ldots \ldots \ldots \ldots$ & $25^{\circ}$ & $1.26 \times 10^{-3}$ \\
\hline Triethylamine $\ldots \ldots \ldots \ldots \ldots \ldots \ldots \ldots \ldots \ldots \ldots \ldots$ & $25^{\circ}$ & $6.4 \times 10^{-4}$ \\
\hline 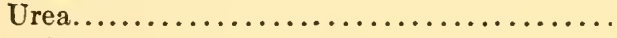 & $25^{\circ}$ & $1.5 \times 10^{-14}$ \\
\hline Aniline $\ldots \ldots \ldots \ldots \ldots \ldots \ldots \ldots \ldots \ldots \ldots \ldots$ & $25^{\circ}$ & $4.6 \times 10^{-10}$ \\
\hline Guanine..$\ldots \ldots \ldots \ldots \ldots \ldots \ldots \ldots$ & $40^{\circ}$ & $8.4 \times 10^{-12}$ \\
\hline Caffeine.................. & $40^{\circ}$ & $4.1 \times 10^{-11}$ \\
\hline Creatinine................. & $40^{\circ}$ & $3.7 \times 10^{-9}$ \\
\hline 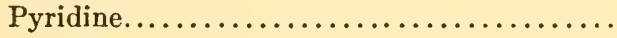 & $25^{\circ}$ & $2.3 \times 10^{-14}$ \\
\hline 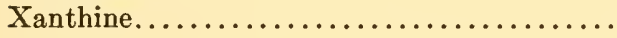 & $40^{\circ}$ & $4.8 \times 10^{-14}$ \\
\hline
\end{tabular}

acids and will be dealt with later together with them. Also the slightly soluble bases, such as $\mathrm{Cu}(\mathrm{OH})_{2}, \mathrm{AgOH}$ are probably to be classed with the strong, greatly dissociated bases. The fact that they do not produce in water a marked alkaline reaction is to be attributed exclusively to the slightness of their solubility but not to any lack of dissociation. A base such as $\mathrm{AgOH}$ is not known in its free, pure state. But it is highly probable that the traces of $\mathrm{Ag}_{2} \mathrm{O}$ which dissolve in water go into solution entirely as $\mathrm{AgOH}$, and that the latter is practically completely dissociated, so that actually almost only $\mathrm{Ag}^{+}$and $\mathrm{OH}^{-}$ions are present in solution. 
Of the organic bases only the quartenary ones are comparable to those above. The other organic bases, especially the important ones, such as the amino- and oxonium-bases, as well as some others, are to be regarded as pseudo-bases. It was shown earlier in this chapter how the basic character of $\mathrm{NH}_{3}$ may be explained either by the hypothesis of the formation of an extremely small amount of $\mathrm{NH}_{4} \mathrm{OH}$ and its subsequent dissociation, or, in a simpler way, by means of the addition reaction $\mathrm{NH}_{3}+\mathrm{H}^{+} \rightarrow \mathrm{NH}_{4}{ }^{+}$. The same holds true for all other weak bases. In table 9 the (apparent) dissociation constants of some weak bases are given.

As it was stated before, the dissociation constants of all organic bases are "apparent." Nothing is experimentally known of the true dissociation constant of $\mathrm{NH}_{4} \mathrm{OH}$. But it will not be far amiss to include it among the quite strong bases in analogy with the alkali metal hydroxides. Ammonia, $\mathrm{NH}_{3}$, possesses as a base an apparently very small constant only because it forms in solution immeasurably small amounts of $\mathrm{NH}_{4} \mathrm{OH}$. But the $\mathrm{NH}_{4} \mathrm{OH}$ is best considered as being almost completely dissociated.

\section{The hydrogen ion concentration in pure acid solutions}

Thus far it has been established in a purely qualitative sense that acids increase the hydrion concentration of water and that bases decrease it. We shall now pass to the quantitative relations existing between the $\left[\mathrm{H}^{+}\right]$and the kind of acid, its dissociation constant and its concentration. Given a solution of an acid in water, then the concentration of its acid ions must be equal to that of the $\mathrm{H}^{+}$ions. Therefore, we can substitute in equation (1) on page $29\left[\mathrm{H}^{+}\right]=\left[\mathrm{S}^{-}\right]$and obtain the expression

$$
\frac{\left[\mathrm{H}^{+}\right]^{2}}{[\mathrm{HS}]}=\mathrm{k}
$$

or,

$$
\left[\mathrm{H}^{+}\right]=\sqrt{\mathrm{k}[\mathrm{HS}]}
$$

Let us assume that A moles of an acid are dissolved in one liter of solution. Part of the acid is dissociated into H-ions and acid ions, the rest of it remaining undissociated. The concentration of 
the unionized part is $[\mathrm{SH}]=[\mathrm{A}]-\left[\mathrm{H}^{+}\right]$. From which it follows that

$$
\begin{gathered}
\frac{\left[\mathrm{H}^{+}\right]^{2}}{[\mathrm{~A}]-\left[\mathrm{H}^{+}\right]}=\mathrm{k} \\
{\left[\mathrm{H}^{+}\right]=\sqrt{\frac{\mathrm{k}^{2}}{4}+\mathrm{kA}}-\frac{\mathrm{k}}{2}}
\end{gathered}
$$

For weak acids this formula is simplified in the following manner:

When the dissociation constant of the acid is very small then its ionization is naturally very slight, less than one per cent, for example. It can then be said with a fair degree of accuracy that the concentration of the undissociated acid is equal to that of the total acid, or, $[\mathrm{HS}]=[\mathrm{A}]$. Hence it follows that

$$
\left[\mathrm{H}^{+}\right]=\sqrt{\mathrm{k} \times[\mathrm{A}]}
$$

The $\left[\mathrm{H}^{+}\right]$is, therefore, proportional to the square root of the concentration of the total acid. The analogous rule holds for the case of a weak base where

$$
\left[\mathrm{OH}^{-}\right]=\sqrt{\mathrm{k} \times[\mathrm{B}]}
$$

where $[B]$ is the concentration of the total base. By substituting for $\left[\mathrm{OH}^{-}\right]$its value as $\frac{k_{\mathrm{w}}}{\left[\mathrm{H}^{+}\right]}$the relation becomes

$$
\left[\mathrm{H}^{+}\right]=\frac{\mathrm{k}_{\mathrm{w}}}{\sqrt{\mathrm{k} \times[\mathrm{B}]}}
$$

For the weakest acids whose dissociation constants are of the order of magnitude of that of water, as well as for moderately strong acids in extremely low concentrations the above formulas do not hold. For in these cases the $\mathrm{H}$-ions yielded by the water cannot be ignored in relation to those of the acid. In such cases the $\left[\mathrm{H}^{+}\right]$is determined in the following way: Here also as above, because of the very slight degree of dissociation $[\mathrm{HS}]=[\mathrm{A}]$ and

$$
\frac{\left[\mathrm{H}^{+}\right]\left[\mathrm{S}^{-}\right]}{[\mathrm{A}]}=\mathrm{k}
$$

Further, in accord with the law of electro-neutrality which 
demands that the sum of the total positive ions be equal to the sum of the total negative ions:

$$
\left[\mathrm{H}^{+}\right]=\left[\mathrm{S}^{-}\right]+\left[\mathrm{OH}^{-}\right]
$$

and thirdly,

$$
\left[\mathrm{OH}^{-}\right]=\frac{\mathrm{k}_{\mathrm{w}}}{\left[\mathrm{H}^{+}\right]}
$$

By eliminating $\left[\mathrm{S}^{-}\right]$in equation (2) by using (1), and then substituting for $\left[\mathrm{OH}^{-}\right]$by the use of (3), we obtain

$$
\left[\mathrm{H}^{+}\right]=\frac{k[\mathrm{~A}]}{\left[\mathrm{H}^{+}\right]}+\frac{\mathrm{k}_{\mathrm{w}}}{\left[\mathrm{H}^{+}\right]}
$$

or

$$
\left[\mathrm{H}^{+}\right]=\sqrt{\mathrm{k}[\mathrm{A}]+\mathrm{k}_{\mathrm{w}}}
$$

It is to be observed from the above that the $\left[\mathrm{H}^{+}\right]$may never become less than $k_{w}$, or below true neutrality, not even with an infinitely small $\mathrm{k}$; and also that acids whose $\mathrm{k}$ differs very little from $k_{w}$ raise the $\left[\mathrm{H}^{+}\right]$of water to so slight an extent that solutions of sugar, for instance, are to be considered as being virtually "neutral." The acid nature of sugar becomes evident only in its ability to raise the $\left[\mathrm{H}^{+}\right]$of solutions of bases, in which case the $\left[\mathrm{OH}^{-}\right]$concentration is decreased without the sugar actually "binding" the $\mathrm{NaOH}$ molecule.

In a mixture of two weak acids, whose total concentrations are $A_{1}$ and $A_{2}$, the concentrations of undissociated molecules are $S_{1}$ and $S_{2}$ and the dissociation constants are $k_{1}$ and $k_{2}$ respectively, the $\left[\mathrm{H}^{+}\right]$may be calculated in the following way:

$$
\begin{array}{ll}
\text { 1. } & {\left[\mathrm{S}_{1}^{-}\right]+\left[\mathrm{S}_{2}^{-}\right]=\left[\mathrm{H}^{+}\right]} \\
\text {2. } & \frac{\left[\mathrm{S}_{1}^{-}\right]\left[\mathrm{H}^{+}\right]}{\left[\mathrm{S}_{1}\right]}=\mathrm{k}_{1} \\
\text { 3. } & \frac{\left[\mathrm{S}_{2}^{-}\right]\left[\mathrm{H}^{+}\right]}{\left[\mathrm{S}_{2}\right]}=\mathrm{k}_{2}
\end{array}
$$

Hence

$$
\left[\mathrm{H}^{+}\right]=\sqrt{\mathrm{k}_{1} \mathrm{~S}_{1}+\mathrm{k}_{2} \mathrm{~S}_{2}}
$$


But since the concentrations of the undissociated acids, $\left[\mathrm{S}_{1}\right]$ and $\left[\mathrm{S}_{2}\right]$, in the case of weak acids are virtually equal to those of the total acids, or to $\left[A_{1}\right]$ and $\left[\Lambda_{2}\right]$ respectively, the above expression becomes for practical purposes:

$$
\left[\mathrm{H}^{+}\right]=\sqrt{\mathrm{k}_{1} \mathrm{~A}_{1}+\mathrm{k}_{2} \mathrm{~A}_{2}}
$$

For example if we take a solution containing one mole of acetic acid per liter $\left(\mathrm{k}_{1}=2 \times 10^{-5}\right)$ and one mole of carbonic acid $\left(\mathrm{k}_{2}\right.$ $=3 \times 10^{-7}$ ), then its hydrion concentration is

$$
\left[\mathrm{H}^{+}\right]=\sqrt{2 \times 10^{-5}+3 \times 10^{-7}}
$$

which is practically equal to that of a one molar acetic acid solution without carbonic acid, where

$$
\left[\mathrm{H}^{+}\right]=\sqrt{2 \times 10^{-5}}
$$

Even the hydrion concentration of a solution of one molar $\mathrm{CO}_{2}$ +0.1 molar acetic acid solution.

$$
\left[\mathrm{H}^{+}\right]=\sqrt{0.1 \times 2 \times 10^{-5}+3 \times 10^{-7}}=4.5 \times 10^{-3}
$$

is practically the same as that of a pure $0.1 M$ acetic solution where $\left[\mathrm{H}^{+}\right]=4.48 \times 10^{-3}$. This illustrates the fact that the stronger acid suppresses the dissociation of a weaker acid.

Many years ago Arrhenius ${ }^{29}$ established the law which states: When isohydric solutions (solutions of the same $\left[H^{+}\right]$) of different acids are mixed, the $\left[\mathrm{H}^{+}\right]$is not changed on mixing. This law may be easily derived from the data given above in this section. Let $A_{1}$ and $A_{2}$ be the concentrations of the two acids whose dissociation constants are $k_{1}$ and $k_{2}$. The concentrations $A_{1}$ and $A_{2}$ are so chosen that the $\left[\mathrm{H}^{+}\right]$of the two solutions is the same. This condition is fulfilled when (see (1a) on p. 40) $\mathrm{k}_{1} \times \mathrm{A}_{1}=\mathrm{k}_{2} \times \mathrm{A}_{2}$, and then of course $\left[\mathrm{H}^{+}\right]=\sqrt{\mathrm{k}_{1} \times \mathrm{A}_{1}}=\sqrt{\mathrm{k}_{2} \times \mathrm{A}_{2}}$. Let us now $\operatorname{mix} \mathrm{m}$ volumes of the first solution with $\mathrm{n}$ volumes of the second. Then the concentration of the first acid in the mixture is $\frac{m}{m+n} A_{1}$, and the concentration of the second is $\frac{n}{m+n} A_{2}$. According to (5) above the hydrion concentration of the mixture is:

$$
\left[\mathrm{H}^{+}\right]=\sqrt{\mathrm{k}_{1} \cdot \frac{\mathrm{m}}{\mathrm{m}+\mathrm{n}} \mathrm{A}_{1}+\mathrm{k}_{2} \frac{\mathrm{n}}{\mathrm{m}+\mathrm{n}} \mathrm{A}_{2}}
$$

${ }^{29}$ Sv. Arrhenius, Zeitschr. f. physikal. Chem. 2, 284 (1888). 
Since $\mathrm{k}_{1} \mathrm{~A}_{1}=\mathrm{k}_{2} \mathrm{~A}_{2}$,

$$
\left[\mathrm{H}^{+}\right]=\sqrt{\mathrm{k}_{1} \mathrm{~A}_{1}\left(\frac{\mathrm{m}}{\mathrm{m}+\mathrm{n}}+\frac{\mathrm{n}}{\mathrm{m}+\mathrm{n}}\right)}=\sqrt{\mathrm{k}_{1} \mathrm{~A}_{1}}
$$

and $\left[\mathrm{H}^{+}\right]$is therefore unchanged. But this law is only valid for mixtures of the free acids alone in the absence of their salts. It is not applicable to two isohydric solutions of any composition.

\section{Mixtures of weak acids and their alkali salts: Regulators or Buffers}

While it is true that acids and bases are capable of presenting all possible gradations of dissociation, this is not the case for their alkali salts. The latter all belong to the class of strong electrolytes to which the law of mass action is not applicable without further amplification. The same is true of the chlorides (bromides, etc.) of the weak bases. For the sake of simplifying calculations we may assume at once that the salts are completely dissociated under all conditions. This assumption is but very little removed from reality, and the results which are to be derived from it possess quite a high degree of accuracy and form the foundation of the theory of regulators or buffers. ${ }^{30}$

When to a solution of a weak acid its alkali salt is added, the dissociation of the acid becomes greatly depressed, and to a progressively greater extent with increasing amounts of the salt. Thus by adding varying amounts of sodium acetate to acetic acid it is possible to obtain at will any low hydrion concentration. The same results may be obtained with a great variety of other such acidsalt mixtures. The calculation of the $\left[\mathrm{H}^{+}\right]$of such mixtures is of special importance and is met with very frequently in experimental procedures. Let us take for example a mixture of acetic acid and sodium acetate. The expression applicable to all such cases is:

$$
\frac{\left[\mathrm{S}^{-}\right] \times\left[\mathrm{H}^{+}\right]}{[\mathrm{SH}]}=\mathrm{k}
$$

${ }^{30}$ Literature: Contributions from Nernst's Laboratory: Fels. Zeitschr. f. Elektrochem. 10, 208 (1904); Salesski, ibid. 10,204 (1904). Also S. P. L. Sørensen, Biochem. Zeitschr. 21, 131 (1909); Lawrence J. Henderson, Ergebn. d. Physiol. 8, 254 (1909); L. Michaelis and P. Rona, Biochem. Zeitschr. 23, 364 (1910). 
from which

$$
\left[\mathrm{H}^{+}\right]=\frac{\mathrm{k}[\mathrm{SH}]}{\left[\mathrm{S}^{-}\right]}=\mathrm{k} \frac{\text { [undissociated acid] }}{[\text { acid ion] }}
$$

In such a mixture the concentration of the undissociated acid is virtually equal to the concentration of total free acetic acid, for the latter is very slightly dissociated. The concentration of the anions in this mixture is virtually equal to that of the sodium acetate, for the latter is assumed to be completely dissociated, and the acetate ions given off by the free acetic acid are negligible in quantity compared to those given off by the salt. The expression given above therefore becomes:

$$
\left[\mathrm{H}^{+}\right]=\mathrm{k} \times \frac{[\text { free acetic acid }]}{[\mathrm{Na}-\text { acetate }]}
$$

This expression is an approximation, since it is based on certain assumptions which are not absolutely exact. But it is quite valid for a great variety of cases with a very fair degree of accuracy. The deviations found under certain conditions, which will be dwelt upon later, are so small that they can well be used only as corrections for the above approximation.

For somewhat stronger acids, such as tartaric acid, in which the dissociation of the free acid may not be disregarded, the concentration of the undissociated acid is not assumed to be equal to that of the free acid $[\mathrm{E}]$ but is expressed as $[\mathrm{E}]-\left[\mathrm{H}^{+}\right]$; and the concentration of the anions is not equal to that of the salt, for the free acid yields also anions to the same extent as it does H-ions. Instead of the above equation (1) we obtain:

$$
\left[\mathrm{H}^{+}\right]=\frac{\mathrm{k}\left([\mathrm{E}]-\left[\mathrm{H}^{+}\right]\right)}{[\mathrm{Na} \text {-salt }]+\left[\mathrm{H}^{+}\right]}
$$

From this the following quadratic equation for $\left[\mathrm{H}^{+}\right]$is derived:

$$
\left[\mathrm{H}^{+}\right]=-\frac{[\mathrm{Na}-\mathrm{sal} t]+\mathrm{k}}{2}+\sqrt{\left(\frac{[\mathrm{Na}-\mathrm{salt}]+\mathrm{k}}{2}\right)^{2}+\mathrm{k}[\mathrm{E}]}
$$

This is the first correction for the approximate formula. Practically, it is seldom of any significance. The second much more important correction has the purpose of rectifying the approximate 
assumption of the total dissociation of the Na-salt. This point will be considered in the discussion of the strong electrolytes.

Analogously, in the case of a mixture of $\mathrm{NH}_{3}+\mathrm{NH}_{4} \mathrm{Cl}$ the following approximate expression holds good:

$$
\left[\mathrm{OH}^{-}\right]=\mathrm{k} \frac{\left[\mathrm{NH}_{3}\right]}{\left[\mathrm{NH}_{4} \mathrm{Cl}\right]}
$$

The following method may be used for the experimental proof of equation (1): If in a series of solutions the concentration of the sodium acetate be kept constant, at $0.1 N$ for example, and the concentration of the free acetic acid be varied, it will be observed that within wide limits this variation will not show any significant deviations from the postulated law. But the verification of the formula will not be so complete, if the above conditions be reversed,

TABLE 10

\begin{tabular}{c|c|c}
\hline Dilution & \multicolumn{1}{|c}{$\left[\mathrm{H}^{+}\right]$} & $\mathrm{pH}$ \\
\cline { 2 - 3 } 1 & $1.51 \times 10^{-7}$ & 6.813 \\
$1 / 2$ & $1.25 \times 10^{-7}$ & 6.904 \\
$1 / 5$ & $1.02 \times 10^{-7}$ & 6.990 \\
$1 / 20$ & $0.853 \times 10^{-7}$ & 7.069 \\
$1 / 50$ & $0.817 \times 10^{-7}$ & 7.088 \\
$\infty$ & $0.80 \times 10^{-7}$ & 7.10 (extrapolated) \\
\hline
\end{tabular}

and the concentration of the salt be varied with a constant concentration of the free acid. In the latter case it will be found that the dissociation of the sodium acetate which had been assumed to be always complete, varies somewhat with its concentration. This condition becomes noticeable when a given mixture of acetic acid and its sodium salt is diluted with water. For a solution of $0.1 \mathrm{~N}$ acetic acid $+0.1 N$ sodium acetate the following figures were found by the gas chain method:

\begin{tabular}{|c|c|c|}
\hline & {$\left[\mathrm{H}^{+}\right]$} & $\mathrm{pH}$ \\
\hline Undiluted............... & $2.42 \times 10^{-5}$ & 4.615 \\
\hline Diluted $\times 5 \ldots \ldots \ldots \ldots \ldots \ldots \ldots$ & $2.16 \times 10^{-5}$ & 4.665 \\
\hline
\end{tabular}

Thus the effect of dilution is just barely demonstrable. In any case it is so slight that equation (1) may be regarded for all practical purposes as quite accurate and valid. 
The deviation from this approximate equation is more marked in the case of a solution of a mixture of primary and secondary sodium phosphates. If these salts be assumed to be totally dissociated, then for a mixture of their solutions it would be expected that

$$
\left[\mathrm{H}^{+}\right]=\mathrm{k} \frac{\text { [primary phosphate] }}{\text { [secondary phosphate] }}
$$

But it is to be remembered that the secondary phosphate is the Na-salt of the weak acid-"primary phosphate." If the above expression were entirely correct, then the absolute concentration of the phosphates would be immaterial. This is true to a certain extent, but not in so strictly proportional a manner as in the case of the acetate mixtures, for the degree of dissociation of dibasic phosphate depends to a much greater extent upon the dilution. Electrometric determinations of the $\left[\mathrm{H}^{+}\right]$of a mixture of equal parts of $M / 15 \mathrm{~K}_{2} \mathrm{HPO}_{4}+M / 15 \mathrm{NaH}_{2} \mathrm{PO}_{4}$ yielded the figures in table $10 .^{31}$

\section{The two-acids problem}

It is frequently desired to calculate the $\left[\mathrm{H}^{+}\right]$obtaining in a mixture of two weak acids and of its alkali salts. This particular condition was designated as the two-acids problem. ${ }^{31}$ A general solution of this problem is quite difficult and is barely to be found. But since this problem has a practical bearing, as will be seen later, it will be here detailed for certain simple conditions which should suffice for the handling of any practical question which may arise.

For this purpose the derivation given in the preceding section will be modified, and we shall return once again to the mixture of a weak acid and its alkali salt. Such a mixture may also be obtained by mixing a weak acid of concentration A with a strong base of concentration $L$, where $L<A$. In such mixture then the concentration of the salt $=\mathrm{L}$ and that of the free acid in excess $=\mathrm{A}-\mathrm{L}$. Then the equation (1) on page 44 may be transformed into

$$
\left[\mathrm{H}^{+}\right]=\mathrm{k} \frac{\mathrm{A}-\mathrm{L}}{\mathrm{L}}
$$

Similarly a mixture of two acids and their salts may be thought of

${ }^{31}$ L. Michaelis and A. Krüger, Biochem. Zeitschr. 119, 307 (1921). 
as being produced by mixing the two acids, whose dissociation constants are $k_{1}$ and $k_{2}$ and whose concentrations are $A_{1}$ and $A_{2}$ respectively, with a base whose total concentration is $\mathrm{L}$, where $\mathrm{L}<\left(\mathrm{A}_{1}\right.$ $+A_{2}$ ). Of this amount of base a part $L_{1}$ is bound by the first acid and a part $L_{2}$ by the second, in such a manner that

$$
\mathrm{L}_{1}+\mathrm{L}_{2}=\mathrm{L}
$$

In accord with the mass law:

$$
\begin{aligned}
& {\left[\mathrm{H}^{+}\right]=\mathrm{k}_{1} \frac{A_{1}-\mathrm{L}_{1}}{\mathrm{~L}_{1}}} \\
& {\left[\mathrm{H}^{+}\right]=\mathrm{k}_{2} \frac{A_{2}-\mathrm{L}_{2}}{\mathrm{~L}_{2}}}
\end{aligned}
$$

It is now necessary to recollect that equations (1) as well as (1a) are only approximations and may be only applied when the second fractional factor on the right side has a value lying between 0.01 and 100. In further calculations the conditions under which they are applicable will always be borne in mind. If now in (1c) we take $L_{1}=\mathrm{L}-\mathrm{L}_{2}$ and eliminate $\mathrm{L}_{2}$ by means of (1d) a quadratic equation for $\left[\mathrm{H}^{+}\right]$is obtained which is capable of solution only in a physical sense:

$$
\begin{aligned}
{\left[\mathrm{H}^{+}\right]=} & \frac{\mathrm{k}_{1}\left(A_{1}-\mathrm{L}\right)+\mathrm{k}_{2}(\mathrm{~A}-\mathrm{L})}{2 \mathrm{~L}} \\
& +\sqrt{\left[\frac{\mathrm{k}_{1}\left(A_{1}-L\right)+\mathrm{k}_{2}\left(A_{2}-L\right)}{2 L}\right]^{2}+\frac{k_{1} \cdot k_{2}}{L}\left(A_{1}+A_{2}-L\right)}
\end{aligned}
$$

The problem under consideration has the following useful practical application. In the determination of $\left[\mathrm{H}^{+}\right]$by indicator methods we add to the given solution some indicator, which is an acid, and we tacitly assume that this addition of acid does not alter the $\left[\mathrm{H}^{+}\right]$of our solution. The solution in question is as a rule of the nature of a single acid buffer, such as, for example, a mixture of $\mathrm{NaHCO}_{3}+\mathrm{H}_{2} \mathrm{CO}_{3}$. Let us carry out the calculation for a most extreme case. Let the solution in question be extremely poor in buffer value, such as ordinary water + conductivity water. Such a solution may be obtained by mixing $0.00300 \mathrm{~N}$ calcium bicarbonate $\left(\mathrm{NaHCO}_{3}\right.$ will do as well) and $0.00030 \mathrm{~N} \mathrm{H}_{2} \mathrm{CO}_{3}$. In this case $\mathrm{L}$ 
$=0.003, \mathrm{~A}_{1}=0.0033$ and $\mathrm{k}_{1}$ is given as $=3 \times 10^{-7}$. We now add to this solution an indicator, $m$-nitrophenol $\left(\mathrm{k}_{2}=5 \times 10^{-9}\right)$ in the concentration of $\mathrm{A}_{2}=0.003 \mathrm{~N}$. In reality barely one half of this amount is actually needed, although, as it happens, $m$-nitrophenol possesses weak tinctorial power, and much more of this dye is required than in the case of the other indicators. If the $\left[\mathrm{H}^{+}\right]$ of the solution before the addition of the indicator is calculated from (1a), then we obtain on the basis of the given data the value $\left[\mathrm{H}^{+}\right]$ $=3 \times 10^{-8}$. After the addition of the indicator we obtain by the use of $(1 \mathrm{e})\left[\mathrm{H}^{+}\right]=5.83 \times 10^{-8}$.

In a similar way, assuming various values for the concentration of the indicator, $A_{2}$, and retaining all the other data, the figures in table 11 are obtained.

TABLE 11

\begin{tabular}{l|c|c|c}
\hline $\mathrm{A}_{2}$ (mols perliter) & {$\left[\mathrm{H}^{+}\right]$} & $\mathrm{pH}$ & $\begin{array}{c}\text { Error in pH due to } \\
\text { indicator }\end{array}$ \\
\hline 0.003 & $5.83 \times 10^{-8}$ & 7.23 & -0.29 \\
0.0015 & $4.66 \times 10^{-8}$ & 7.33 & -0.20 \\
0.00075 & $3.93 \times 10^{-8}$ & 7.41 & -0.11 \\
0.00037 & $3.46 \times 10^{-8}$ & 7.46 & -0.06 \\
0.00018 & $3.27 \times 10^{-8}$ & 7.49 & -0.03 \\
0 & $3.0 \times 10^{-8}$ & 7.52 & \\
\hline
\end{tabular}

In using $m$-nitrophenol one employs, for example, a 0.3 per cent, or, 0.027 molar solution. In adding $0.3 \mathrm{cc}$. to $20 \mathrm{cc}$. of conductivity water the concentration of the indicator becomes in round numbers $0.0004 \mathrm{~N}$, and the $\mathrm{pH}$ error due to the indicator is -0.06 , a value falling well within the experimental limits of error. In reality much smaller amounts of indicator suffice. In the case of all other indicators which have greater coloring powers and which can be used in much smaller concentrations this error is certainly of no significance.

\section{The degree of dissociation and the dissociation-residue of acids}

The degree of dissociation of an acid in a pure aqueous solution is the term given to the ratio of the dissociated portion to the total amount of the acid. This definition will be extended so that it may also embrace the acid-salt mixtures. Restating then, by the degree of dissociation of an acid we understand in general the ratio 
of the acid ions to the total amount of acid radicals, irrespective of the form in which they may happen to occur. The degree of dissociation of acetic acid in a mixture of acetic acid and its Na-salt is, therefore, represented by the ratio of the acetate anions to the total amount of acetate radicals in the free acid as well as in the Na-salt. The dissociation residue ${ }^{32}$ is represented by the ratio of the undissociated (acetic) acid radicals to the total amount of (acetic) acid radicals present. These two conceptions will be repeatedly utilized in our further discussions. With such applications in mind, the question arises: What is the relation of the degree of dissociation and of the dissociation-residue to the hydrion concentration? The degree of dissociation (as well as the residue) is considered as a function of $\left[\mathrm{H}^{+}\right]$, and thus, contrary to the foregoing procedure, the $\left[\mathrm{H}^{+}\right]$is treated as an independent variable. Since in the acid-salt mixtures we have found the ready means of obtaining any desired $\left[\mathrm{H}^{+}\right]$for any practical purpose, the assumption of $\left[\mathrm{H}^{+}\right]$being an independent variable may now appear as quite conceivable.

According to the above definition, the degree of dissociation $\alpha$ may be expressed as

$$
\alpha=\frac{\left[\mathrm{S}^{-}\right]}{[\mathrm{A}]}
$$

where $\mathrm{S}^{-}$as previously represents the acid anions and $\mathrm{A}$ the total acid in whatever form present. Let us now recall the equation on page 43.

$$
\left[\mathrm{H}^{+}\right]=\frac{\mathrm{k}[\mathrm{HS}]}{\left[\mathrm{S}^{-}\right]}
$$

According to the definition the degree of dissociation $\alpha=\frac{\left[\mathrm{S}^{-}\right]}{[\mathrm{A}]}$ and $[\mathrm{A}]=[\mathrm{HS}]+\left[\mathrm{S}^{-}\right]$

Therefore,

$$
\alpha=\frac{\left[\mathrm{S}^{-}\right]}{[\mathrm{HS}]+\left[\mathrm{S}^{-}\right]}
$$

${ }^{32}$ L. Michaelis, Biochem. Zeitschr. 33, 182 (1911). 
By substituting for [HS] its value in (1), namely, $\frac{\left[\mathrm{H}^{+}\right]\left[\mathrm{S}^{-}\right]}{\mathrm{k}}$, we obtain

$$
\alpha=\frac{\mathrm{k}}{\mathrm{k}+\left[\mathrm{H}^{+}\right]}
$$

Thus this equation represents $\alpha$ as a function of $\left[\mathrm{H}^{+}\right]$. The dissociation constant of the acid, $\mathrm{k}$, appears in this equation as a constant value characterizing the function, which will be here designated as the parameter of this function. The function $\alpha=$ $\frac{\mathrm{k}}{\mathrm{k}+\left[\mathrm{H}^{+}\right]}$is graphically represented by the curve in figure 2 which shows a hyperbola cutting the zero ordinate at a sharp angle.

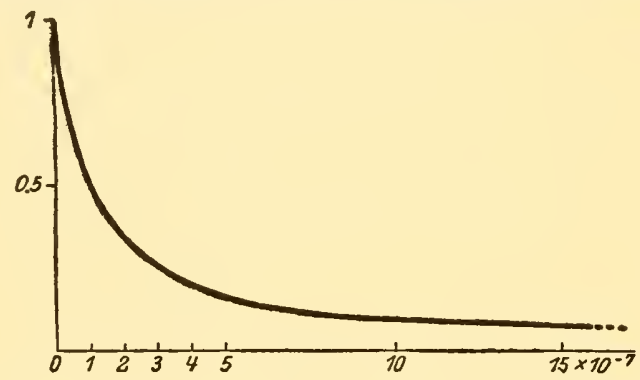

FIG. 2. The degree of dissociation- $(\alpha)$-curve of an acid whose dissociation constant $=1 \times 10^{-7}$. Abscissa $-\left[\mathrm{H}^{+}\right]$, ordinate- $\alpha$.

This representation is not especially clear, and the highly practical significance of this function rests on the fact that by means of a simple logarithmic transformation of the abscissa a very comprehensible graphic form is obtained. Instead of the abscissa representing the $\left[\mathrm{H}^{+}\right]$in our new form it stands for $\mathrm{pH}$, and the curve shows $\alpha$ as a function of $\mathrm{pH}$.

$\mathrm{pH}$ is an antibatic function of $\left[\mathrm{H}^{+}\right]$; i.e., the $\mathrm{pH}$ curve falls with the rising $\left[\mathrm{H}^{+}\right]$curve.

Our function may then be written as

$$
\alpha=\frac{\mathrm{k}}{\mathrm{k}+10^{-\mathrm{pH}}}
$$

and is represented graphically in figure 3 on page 51 . 
The curve is distinguished by the following characteristics:

1. This curve can be divided into three parts: The first and third portions run asymptotically to the $\mathrm{X}$-axis, the first at the height 0 , the third at the height 1 .

2. Between these two portions lies an almost straight-line steep portion which passes into the asymptotes at sharp angles of inflection.

3. Strictly speaking this middle portion is not a straight line, but is slightly curved or S-shaped, and it has a point of inflection at the ordinate height of $\frac{1}{2}$. The projection of this point on the abscissa gives the negative logarithm of the dissociation constant $\mathrm{k}$. If we

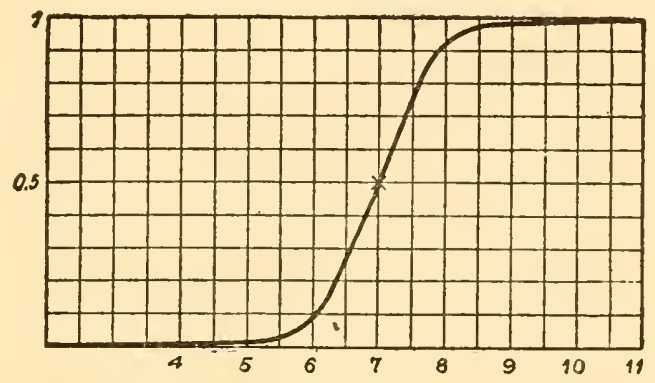

FIG. 3. Dissociation curve of an acid whose constant is $\mathrm{k}=1 \times 10^{-7}$. Abscissa-pH, ordinate- $\alpha$. The scale of the ordinates is enlarged $\times 5$ with respect to the scale of the abscissa.

At the point marked $\mathrm{x} \alpha=\frac{1}{2}$, and the projection of the ordinate on the abscissa gives the value of $\mathrm{pK}$ (the negative logarithm of the dissociation constant).

state, analogously with the previously used method, $-\log \mathrm{k}=\mathrm{pK}$, then it may be said that the parameter of this function is pk, and that geometrically it means that it denotes that value on the $\mathrm{X}$-axis ( $\mathrm{pH}$-axis) for which the ordinate $=\frac{1}{2}$. If similar dissociation curves be drawn for various values of $\mathrm{pK}$ (therefore for acids of various strengths) then all such curves will be parallel. They may be graphically derived from the given curve by simply moving it horizontally on its abscissa until the projection of its ordinate value of $\frac{1}{2}$ coincides with the value of $\mathrm{pK}$ of the particular acid.

4. Finally, this graphic representation has the special advantage insofar as the dissociation-residue curve is an exact mirror image of the dissociation curve. 
The dissociation-residue $\rho$ is expressed according to the definition as

$$
\begin{aligned}
& \rho=\frac{[\mathrm{HS}]}{[\mathrm{HS}]+\left[\mathrm{S}^{-}\right]}=1-\alpha \\
& \rho=\frac{\left[\mathrm{H}^{+}\right]}{\mathrm{k}+\left[\mathrm{H}^{+}\right]}=\frac{1}{1+\frac{\mathrm{k}}{\left[\mathrm{H}^{+}\right]}}
\end{aligned}
$$

and hence

$$
\rho=\frac{10^{-\mathrm{pH}}}{\mathrm{k}+10^{-\mathrm{pH}}}
$$

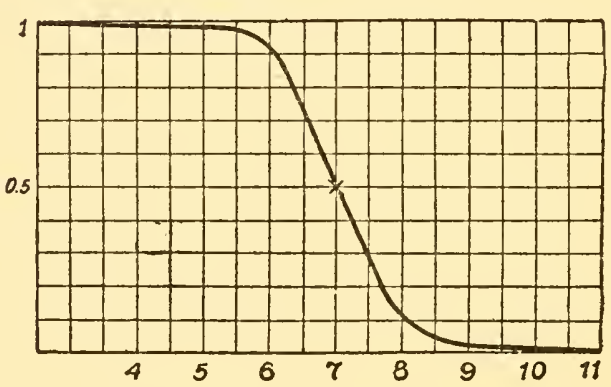

FIG. 4. Dissociation-residue curve of an acid under same conditions as in figure 3. It also represents the dissociation curve of a base whose dissociation constant is $\mathrm{k}=1 \times 10^{-7}$.

The graphic construction is best derived from the relationship $\alpha=1-\rho$, and it is at once perceived from figure 4 that the curve is a mirror image of the $\alpha$-curve, and that its parameter is exactly the same.

The mathematical analysis of these functions is as follows:

Differentiating the function

$$
\alpha=\frac{\mathrm{d}}{\mathrm{k}+\left[\mathrm{H}^{+}\right]}
$$

with respect to $\log \left[\mathrm{H}^{+}\right]$we obtain

$$
\frac{\mathrm{d} \alpha}{\mathrm{d} \log \left[\mathrm{H}^{+}\right]}=\frac{\mathrm{d} \alpha}{\mathrm{d}\left[\mathrm{H}^{+}\right]} \times \frac{\mathrm{d}\left[\mathrm{H}^{+}\right]}{\mathrm{d} \ln \left[\mathrm{H}^{+}\right]} \times \frac{\mathrm{d} \ln \left[\mathrm{H}^{+}\right]}{\mathrm{d} \log \left[\mathrm{H}^{+}\right]}
$$


where $\log$ is the decimal and $\ln$ is the natural logarithm, therefore,

$$
\frac{\mathrm{d} \alpha}{\mathrm{d} \log \left[\mathrm{H}^{+}\right]}=-\frac{\mathrm{k} \times\left[\mathrm{H}^{+}\right]}{\left(\mathrm{k}+\left[\mathrm{H}^{+}\right]^{2}\right)} \cdot \ln 10
$$

and differentiating once more with respect to $\log \left[\mathrm{H}^{+}\right]$we obtain

$$
\begin{gathered}
\frac{d^{2} \alpha}{d \log \left[\mathrm{H}^{+}\right]^{2}}=\frac{\mathrm{d} \frac{\mathrm{d} \alpha}{\mathrm{d} \log \left[\mathrm{H}^{+}\right]}}{\mathrm{d}\left[\mathrm{H}^{+}\right]} \cdot \frac{\mathrm{d}\left[\mathrm{H}^{+}\right]}{\mathrm{d} \ln \left[\mathrm{H}^{+}\right]} \cdot \frac{\mathrm{d} \ln \left[\mathrm{H}^{+}\right]}{\mathrm{d} \log \left[\mathrm{H}^{+}\right]} \\
=-\frac{\mathrm{k} \cdot\left[\mathrm{H}^{+}\right] \cdot\left(\mathrm{k}-\left[\mathrm{H}^{+}\right]\right)}{\left(\mathrm{k}+\left[\mathrm{H}^{+}\right]^{3}\right.} \cdot(\ln 10)^{2}
\end{gathered}
$$

Letting this expression $=0$ we obtain the conditions for the transformation point of the $\alpha$-or $\log \left[\mathrm{H}^{+}\right]$-curve. This condition obtains, as may be readily seen when $\left[\mathrm{H}^{+}\right]=\mathrm{k}$, or $\mathrm{pH}=\mathrm{pK}$.

By substituting in (1) $\left[\mathrm{H}^{+}\right]=\mathrm{k}$, we have $\alpha=\frac{1}{2}$.

Thus at the transformation point,

$$
\begin{aligned}
& \text { 1. } \mathrm{pH}=\mathrm{pK} \\
& \text { 2. } \alpha=\frac{1}{2}
\end{aligned}
$$

which was to be demonstrated.

The same holds true for the residue curve which at the transformation point ordinate is symmetrical with the dissociation curve.

The angle at which the curve intersects the ordinate in the vicinity of the transformation point is of interest. According to the fundamental laws of differential calculus the differential quotient represents the tangent of the unknown angle $\varphi$. Since at the point concerned $\left[\mathrm{H}^{+}\right]=\mathrm{k}$, it follows by substitution in (2) above that

$$
\frac{\mathrm{d} \alpha}{\mathrm{d} \log \left[\mathrm{H}^{+}\right]}=-\frac{\ln 10}{4}=\frac{2.303}{4}=0.576
$$

and $\operatorname{arc} \tan \varphi=$ almost exactly $30^{\circ}$. In order to obtain the correct angle by this method one must take care that in the graphic representation the ordinates are drawn to the same scale as the abscissa. If the scale of the ordinates is $n$ times that of the abscissa then the tangent of the angle of intersection is $\mathrm{n}$ times greater. In the curves of figures 3 and $4, \mathrm{n}=5$, and therefore, the tangent $=2.88$ and the angle of inclination is approximately $71^{\circ}$. 
All of the above applies entirely and directly to the dissociation of the bases, and all that is needed is to substitute $\left[\mathrm{OH}^{-}\right]$for $\left[\mathrm{H}^{+}\right]$ and $\mathrm{pOH}$ for $\mathrm{pH}$.

Therefore for a base,

$$
\alpha=\frac{1}{1+\frac{\left[\mathrm{OH}^{-}\right]}{\mathrm{k}}} \text { and } \rho=\frac{1}{1+\frac{\mathrm{k}}{\left[\mathrm{OH}^{-}\right]}}
$$

But

$$
\left[\mathrm{OH}^{-}\right]=\frac{\mathrm{k}_{\mathrm{w}}}{\left[\mathrm{H}^{+}\right]}
$$

therefore, substituting for $\left[\mathrm{OH}^{-}\right]$above,

$$
\alpha=\frac{1}{1+\frac{k_{\mathrm{w}}}{\mathrm{k}} \times \frac{1}{\left[\mathrm{H}^{+}\right]}} \text {and } \rho=\frac{1}{1+\frac{\mathrm{k}\left[\mathrm{H}^{+}\right]}{\mathrm{k}_{\mathrm{w}}}}
$$

The $\alpha$-curve of a base with a dissociation constant $\mathrm{k}$ is, therefore, identical with the $\rho$-curve of an acid, whose dissociation constant is $\frac{k_{\mathrm{w}}}{\mathrm{k}}$, and the $\rho$-curve of a base of constant $\mathrm{k}$ is identical with the $\alpha$-curve of an acid whose constant is $\frac{\mathrm{k}_{\mathrm{w}}}{\mathrm{k}}$.

A simple verification of the course of a dissociation curve will be found in the following experiment. $p$-Nitrophenol is a weak acid whose ions are yellow and whose undissociated molecule is colorless. If the same small amount of this indicator be added to a series of solutions adjusted to varying $\mathrm{pH}$ values by the acetate buffers, then the degree of dissociation $\alpha$ of the $p$-nitrophenol and simultaneously the intensity of its color will vary with the $\mathrm{pH}$ of the solution. In figure $11, \mathrm{p} .98$, the $\mathrm{pH}$ is given on the abscissa and the intensity of color, or the degree of dissociation $\alpha$, of the dye on the ordinate. The course of the curve corresponds exactly to the theoretical $\alpha$-curve, and, if $\mathrm{pK}$ be determined graphically by the method given above it will be found to have the value of 7.18 , while almost the same value, 7.28 , was observed by means of the conductivity method (calculated for the same temperature, $18^{\circ}$ from the data of Euler and Bolin and also of Lundén). 


\section{The dissociation of polybasic acids}

A dibasic acid may be defined as a molecule possessing two ionogenic H-atoms. The dissociation of each of the two $\mathrm{H}$-ions must be considered separately. Thus oxalic acid is dibasic, but the tendency to dissociate is different in the two H-ions. The acid dissociates in two stages with each of these stages having a distinct dissociation constant. If the acid radical remaining after the removal of both $\mathrm{H}$-atoms be designated as $\mathrm{S}$ and the whole acid molecule as $\mathrm{SH}_{2}$, then the chemical equations representing the two stages are:

$$
\begin{aligned}
& \text { 1st stage: } \mathrm{SH}_{2} \rightarrow \mathrm{SH}^{-}+\mathrm{H}^{+} \\
& \text {2nd stage: } \mathrm{SH}^{-} \rightarrow \mathrm{S}^{-}+\mathrm{H}^{+}
\end{aligned}
$$

To each of these two reactions the mass law applies in the following way:

$$
\begin{aligned}
& \text { 1. } \frac{\left[\mathrm{SH}^{-}\right]\left[\mathrm{H}^{+}\right]}{\left[\mathrm{SH}_{2}\right]}=\mathrm{k}_{1} \\
& \text { 2. } \frac{\left[\mathrm{S}^{=}\right]\left[\mathrm{H}^{+}\right]}{\left[\mathrm{SH}^{-}\right]}=\mathrm{k}_{2}
\end{aligned}
$$

In order to arrive at a clear understanding of dissociation, the conceptions of the degree of dissociation and of dissociation-residue will be discussed also in this connection. Let the concentration of undissociated acid be designated as [A], that of the primary ions as $\left[\mathrm{A}^{-}\right]$and of the secondary ions as $\left[\mathrm{A}^{=}\right]$, then $\alpha_{1}$ the degree of dissociation of the first stage may be defined as

$$
\alpha_{1}=\frac{\left[\mathrm{A}^{-}\right]}{[\mathrm{A}]+\left[\mathrm{A}^{-}\right]+\left[\mathrm{A}^{-}\right]}
$$

and $\alpha_{2}$ the degree of dissociation of the second step as:

$$
\alpha_{2}=\frac{\left[\mathrm{A}^{-}\right]}{[\mathrm{A}]+\left[\mathrm{A}^{-}\right]+\left[\mathrm{A}^{-}\right]}
$$

and the dissociation-residue, $\rho$, as

$$
\rho=\frac{\mathrm{A}}{[\mathrm{A}]+\left[\mathrm{A}^{-}\right]+\left[\mathrm{A}^{-}\right]}
$$


The two dissociation constants are:

$$
\begin{aligned}
& \mathrm{k}_{1}=\frac{\left[\mathrm{A}^{-}\right]\left[\mathrm{H}^{+}\right]}{[\mathrm{A}]} \\
& \mathrm{k}_{2}=\frac{\left[\mathrm{A}^{-}\right]\left[\mathrm{H}^{+}\right]}{\left[\mathrm{A}^{-}\right]}
\end{aligned}
$$

From the above equations it follows:

$$
\begin{aligned}
& \frac{1}{\alpha_{1}}=\frac{[\mathrm{A}]}{\left[\mathrm{A}^{-}\right]}+1+\frac{\left[\mathrm{A}^{-}\right]}{\left[\mathrm{A}^{-}\right]} \\
& \frac{1}{\alpha_{2}}=\frac{[\mathrm{A}]}{\left[\mathrm{A}^{-}\right]}+\frac{\left[\mathrm{A}^{-}\right]}{\left[\mathrm{A}^{-}\right]}+1 \\
& \frac{1}{\rho}=1+\frac{\left[\mathrm{A}^{-}\right]}{[\mathrm{A}]}+\frac{\left[\mathrm{A}^{-}\right]}{[\mathrm{A}]}
\end{aligned}
$$

and through elimination of $[\mathrm{A}],\left[\mathrm{A}^{-}\right]$and $\left[\mathrm{A}^{-}\right]$we finally obtain:33

$$
\begin{aligned}
\alpha_{1} & =\frac{1}{1+\frac{\left[\mathrm{H}^{+}\right]}{\mathrm{k}_{1}}+\frac{\mathrm{k}_{2}}{\left[\mathrm{H}^{+}\right]}} \\
\alpha_{2} & =\frac{1}{1+\frac{\left[\mathrm{H}^{+}\right]}{\mathrm{k}_{2}}+\frac{\left[\mathrm{H}^{+}\right]}{\mathrm{k}_{1} \cdot \mathrm{k}_{2}}} \\
\rho & =\frac{1}{1+\frac{\mathrm{k}_{1}}{\left[\mathrm{H}^{+}\right]}+\frac{\mathrm{k}_{1} \cdot \mathrm{k}_{2}}{\left[\mathrm{H}^{+}\right]}}
\end{aligned}
$$

The curves shown in figures 5, 6 and 7 are graphic representations of these functions having definite values for the two parameters, $\mathrm{k}_{1}$ and $\mathrm{k}_{2}$ and with the $\mathrm{pH}$ as the abscissa. The dissociation-residue or $\rho$-curve is almost exactly identical with that of a monobasic acid, and its deviations from the latter are so slight that they are not noticeable. The primary dissociation curve, $\alpha_{1}$, shows the most marked changes. It belongs to the same type as the $\rho$-curve of an amphoteric electrolyte, which is to be discussed later, inasmuch as it shows a maximum. Just as in the case of the ampholyte $\rho$-curve the kind of the maximum formation of the curve depends upon the

${ }^{33}$ L. Michaelis, in: C. Oppenheimer, Handb. d. Biochem. Ergänzungsband, p. 57 (1913). 


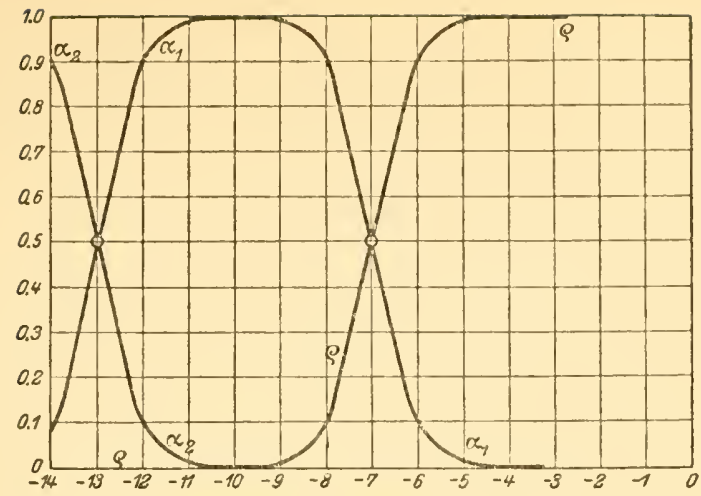

FIG. 5. For the case in which $\mathrm{k}_{1}=10^{-7}, \mathrm{k}_{2}=10^{-12}$

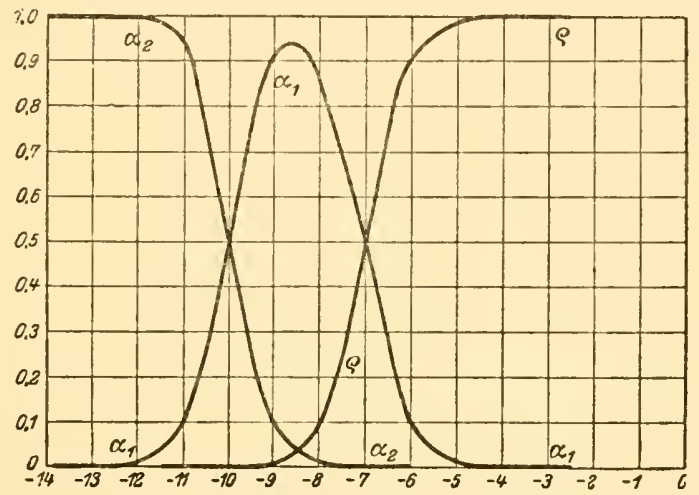

FIG. 6. For the case in which $\mathrm{k}_{1}=10^{-7}, \mathrm{k}_{2}=10^{-10}$

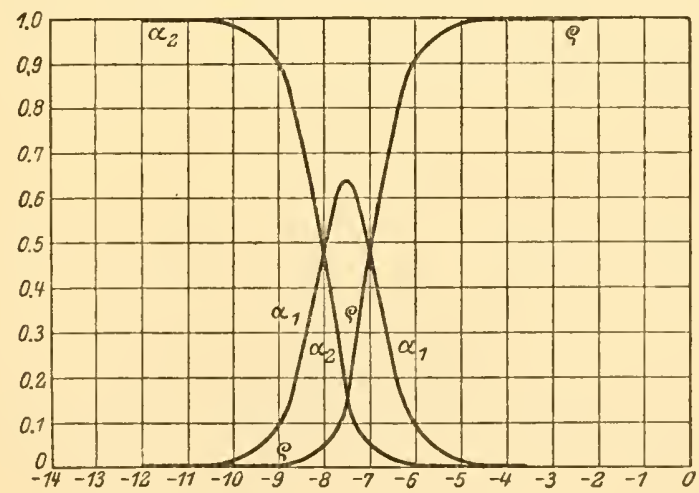

FIG. 7. For the case in which $\mathrm{k}_{1}=10^{-7}, \mathrm{k}_{2}=10^{-8}$

Fıgs. 5 то 7. $\rho$-dissociation-residue curves. $\alpha_{1}$-primary dissociation curves. $\alpha_{2}$-secondary dissociation eurves. Abscissa are log $\left[\mathrm{H}^{+}\right]$. 
values of both dissociation constants of the acid; in fact it depends upon the ratio $k_{1}: k_{2}$. If $k_{1}$ is much greater than $k_{2}$, then a wide rather flat maximum is formed in which $\alpha_{1}$ is almost $=1$ as in figure 5 . When $k_{1}$ is but a little greater than $k_{2}$, as in figure 6 , then the maximum has a sharper angle and it does not quite reach the value 1 ; and when the values of $k_{1}$ and $k_{2}$ are still closer together then these two characteristics are even more pronounced as shown in figure 7. The limiting case is where $k_{1}=k_{2}$, i.e., when the acid is capable of dissociation to the same extent in both stages. In this case $\alpha_{1}$ is always $=0$, which means that only undissociated acid molecules and divalent acid ions are formed and no univalent ions. This last case is not met with in weak electrolytes. The two dissociation constants have more or less differing values. They approach each other quite closely in the case of succinic and fumaric acids, while the two constants differ very widely in maleic and carbonic acids. Wherever the two acid (COOH) groups are spatially close to each other in the molecule, as in maleic acid, then the acid nature is quite pronounced and the acid is strong in the primary stage, while in its second stage only a small residue of acid is present in excess. But if the two $\mathrm{COOH}$-groups are situated far apart, then each behaves in a manner more independent of each and exert a more nearly equal effect.

The position of the maximum in the $\alpha_{1}$-curve is of particular interest. In order to evaluate the maximum it is necessary to differentiate the function $\alpha_{1}=\mathrm{f}\left(\left[\mathrm{H}^{+}\right]\right)$with respect to $\left[\mathrm{H}^{+}\right]$and to set the derivative $=0$. It is much easier to calculate the minimum or the inverse function which is

$$
\frac{1}{\alpha_{1}}=1+\frac{\left[\mathrm{H}^{+}\right]}{\mathrm{k}_{1}}+\frac{\mathrm{k}_{2}}{\left[\mathrm{H}^{+}\right]}
$$

and whose derivative is

$$
\frac{\mathrm{d} \frac{1}{\alpha_{1}}}{\mathrm{~d}\left[\mathrm{H}^{+}\right]}=\frac{1}{\mathrm{k}_{1}}-\frac{\mathrm{k}_{2}}{\left[\mathrm{H}^{+}\right]^{2}}
$$

By setting this derivative $=0$ we obtain the minimum for $\frac{1}{\alpha}$ or the maximum for $\alpha_{1}$

$$
\left[\mathrm{H}^{+}\right]=\sqrt{\mathrm{k}_{1} \times \mathrm{i}_{2}}
$$


which tells us that $\alpha_{1}$ is at its maximum when $\left[\mathrm{H}^{+}\right]$is equal to the geometric mean of the two dissociation constants, or when

$$
\mathrm{pH}=\frac{\mathrm{pk_{1 }}+\mathrm{pk_{2 }}}{2}
$$

The relation of this maximal value becomes evident when it is introduced into the equation for $\alpha_{1}$

$$
\alpha_{1} \max =\frac{1}{1+2 \sqrt{\frac{k_{2}}{k_{1}}}}
$$

TABLE 12

Table of dissociation constants of polybasic acids

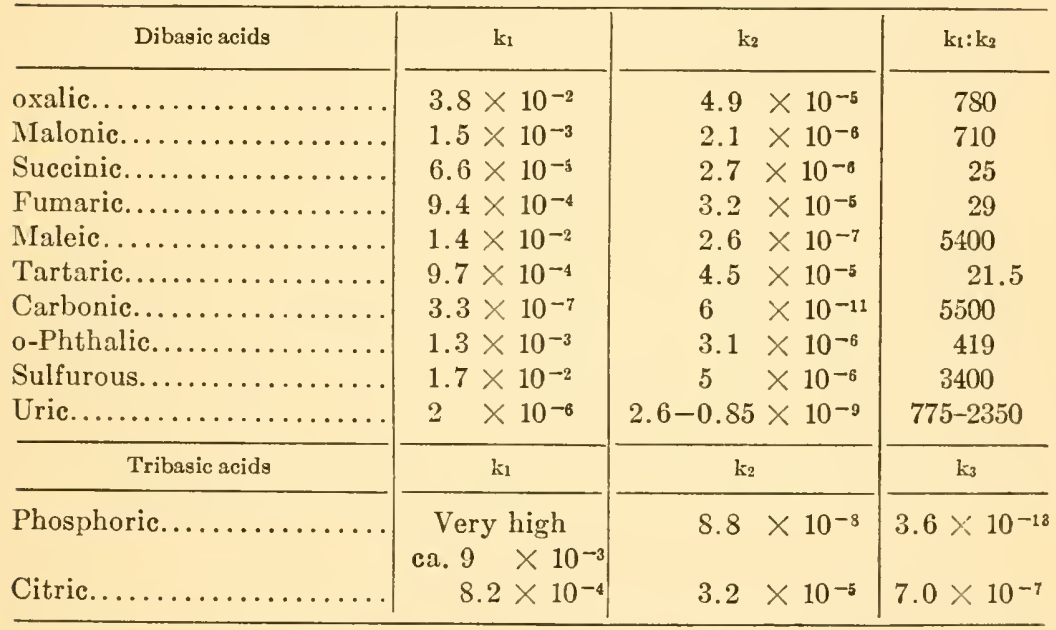

When $2 \sqrt{\frac{k_{2}}{k_{1}}}$ is very much smaller than unity, then the maximal value of $\alpha_{1}$ becomes practically $=1$ and at the same time the maximum of the curve is broad and flat, while in other cases a more pointed maximum is obtained, and its value is less than 1 .

When $\mathrm{k}_{2}$ is much smaller than $\mathrm{k}_{1}$ (fig. 5) the ascending and descending portions of $\alpha_{1}$ represent a part of the dissociation curve and of the dissociation-residue curve as well, and the projections of the two 0.5 ordinates on the abscissa give the values of $\mathrm{pk}_{1}$ and $\mathrm{pk}_{2}$. Both of these points are marked by circles on the curve in figure 5 . 
Furthermore there need be no difficulty in calculating the somewhat complicated functions for polybasic acids. As long as the difference between any two consecutive dissociation constants remains $\bar{₹} 10^{3}$, the curves may be compounded from the individual dissociation and dissociation-residue curves.

The same is true of the polybasic bases, by simply substituting $\left[\mathrm{OH}^{-}\right]$for $\left[\mathrm{H}^{+}\right]$.

As a result of the consecutive dissociation in stages the last ionization stages in polybasic acids can occur only in a strongly alkaline reaction. Thus for example, the concentrations of the carbonate, $\mathrm{CO}_{3}=$, and the tertiary phosphate, $\mathrm{PO} \equiv$ ions are practically negligible in the blood, in which only $\mathrm{HCO}_{3}, \mathrm{HPO}_{4}^{-}$, and $\mathrm{H}_{2} \mathrm{PO}_{4}^{-}$-ions are found.

Table 12 gives the dissociation constants of some dibasic and tribasic acids (chiefly taken from Landolt-Börnstein, Physik. Chem. Tabellen. $k_{2}$ for uric acid is given by A. Kanitz ${ }^{34}$ ).

\section{The dissociation of amphoteric electrolytes ${ }^{35}$}

Amphoteric electrolytes or ampholytes are substances which behave as acids as well as bases. As acids they form salts with bases, and as bases they react with acids to form salts. The ampholytes may be divided into two large groups: those in which the acidic and the basic radicals are spatially apart, and those in which the two radicals are one and identical. To the latter group belong many metal hydroxides. Thus $\mathrm{Zn}(\mathrm{OH})_{2}$ may dissociate either as $\mathrm{Zn}^{++}+2 \mathrm{OH}^{-}$, or as $\mathrm{H}^{+}+\mathrm{ZnO}^{=}$. The most important ampholytes of the first type are the amino-acids. Thus by virtue of its $\mathrm{NH}_{2}$ group glycocoll is a base, while by virtue of its COOH-group it is at the same time an acid. It has long been known that it reacts with $\mathrm{HCl}$ to form a hydrochloride and with $\mathrm{NaOH}$ to form

${ }^{34}$ A. Kanitz, Zeitschr. f. physiol. Chem. 116, 96 (1921).

${ }^{35}$ Literature: G. Bredig, Zeitschr. f. Elektrochem. 6, 33 (1899).-K. Winkelblech, Zeitschr. f. physikal. Chem. 36, 546 (1901).-J. Walker, Zeitschr. f. physikal. Chem. 49, 82 (1904) and 51, 706 (1905).-H. Lundén, K. vetenskap. Nobelinstitut 1 (1908); Arkiv för Kemi, Mineralogi och Geologi (VetenskapsAkad., Stockholm) 2, No. 11; Zeitschr. f. physikal. Chem. 47, 476 (1906).L. Michaelis, Biochem. Zeitschr. 24, 79 and 30, $143(1910) ; 33,182$ (1911) ; 47, 250 (1912); Nernst-Festschrift, 308 (1913); Biochem. Zeitschr. 103, 225 and 106, 83 (1920).--S. P. L. Sörensen, Ergebn. d. Physiol. 12, 303 (1912). 
the sodium salt. But it displays its basic nature to its full extent in a strongly acid solution and its acid nature in a strongly alkaline solution. In pure solution it behaves as a very weak electrolyte and shows a just barely demonstrable acid reaction, because of a predominance of the acidic property of the $\mathrm{COOH}$-group over the basic property of the $\mathrm{NH}_{2}$-group. Assuming that the ampholyte is present in solution in concentration [A], as an acid it forms anions of $\left[\mathrm{A}^{-}\right]$concentration, and its acid dissociation constant is $\mathrm{k}_{\mathrm{a}}$. At the same time in the same solution it also functions as a base whose dissociation constant is $k_{\mathrm{b}}$ and forms cations of $\left[\mathrm{A}^{+}\right]$concentration. $\mathrm{k}_{\mathrm{a}}$ and $\mathrm{k}_{\mathrm{b}}$ must be different in value, and either the acidic or the basic character of the ampholyte predominates. Of all known ampholytes the difference between $k_{a}$ and $k_{b}$ is least in hemoglobin.

In discussing the dissociation relations of the ampholytes it is simplest to begin with a consideration of the dissociation residue, $\rho$, which is defined as the ratio of the undissociated portion to the total concentration $[\mathrm{A}]$ of the ampholyte:

$$
\rho=\frac{[\mathrm{A}]-\left[\mathrm{A}^{+}\right]-\left[\mathrm{A}^{-}\right]}{[\mathrm{A}]}
$$

On the other hand the degree of dissociation must be given separately for the cations and the anions. For the anions it is:

$$
\alpha^{-}=\frac{\left[\mathrm{A}^{-}\right]}{[\mathrm{A}]}
$$

and the degree of dissociation of the cations is:

$$
\alpha^{+}=\frac{\left[\mathrm{A}^{+}\right]}{[\mathrm{A}]}
$$

The mass law finds its application in the following relationships:

$$
\left[\mathrm{A}^{-}\right] \times\left[\mathrm{H}^{+}\right]=\mathrm{k}_{\mathrm{u}} \times[\mathrm{U}]
$$

where $U$ is the undissociated portion of the ampholyte, and furthermore,

$$
\left[\mathrm{A}^{+}\right] \times\left[\mathrm{OI}^{-}\right]=\mathrm{k}_{\mathrm{b}} \times[\mathrm{U}]
$$

It follows, therefore,

$$
\begin{aligned}
& {\left[\mathrm{A}^{-}\right]=\mathrm{k}_{\mathrm{a}} \frac{[\mathrm{U}]}{\left[\mathrm{H}^{+}\right]}} \\
& {\left[\mathrm{A}^{+}\right]=\mathrm{k}_{\mathrm{b}} \frac{[\mathrm{U}]}{\left[\mathrm{OH}^{-}\right]}}
\end{aligned}
$$


and

$$
[\mathrm{A}]-\left[\mathrm{A}^{+}\right]-\left[\mathrm{A}^{-}\right]=[\mathrm{U}]=[\mathrm{A}]-\mathrm{k}_{\mathrm{a}} \frac{[\mathrm{U}]}{\left[\mathrm{H}^{+}\right]}-\mathrm{k}_{\mathrm{b}} \frac{[\mathrm{U}]}{\left[\mathrm{OH}^{-}\right]}
$$

From which follows

$$
[\mathrm{U}]=\frac{[\mathrm{A}]}{1+\frac{\mathrm{k}_{\mathrm{a}}}{\left[\mathrm{H}^{+}\right]}+\frac{\mathrm{k}_{\mathrm{b}}}{\left[\mathrm{OH}^{-}\right]}}
$$

and hence the sought value of $\rho$ may be stated as

$$
\frac{[\mathrm{U}]}{[\mathrm{A}]}=\rho=\frac{1}{1+\frac{\mathrm{k}_{\mathrm{a}}}{\left[\mathrm{H}^{+}\right]}+\frac{\mathrm{k}_{\mathrm{b}}}{\left[\mathrm{OH}^{-}\right]}}
$$

substituting for $\left[\mathrm{OH}^{-}\right]$its value as $\frac{k_{\mathrm{w}}}{\left[\mathrm{H}^{+}\right]}$we obtain:

$$
\rho=\frac{1}{1+\frac{k_{\mathrm{a}}}{\left[\mathrm{H}^{+}\right]}+\frac{\mathrm{k}_{\mathrm{b}}}{\mathrm{k}_{\mathrm{w}}}\left[\mathrm{H}^{+}\right]}
$$

In comparing the expression with that for $\alpha_{1}$ in a dibasic acid as given on page 56 , we find them identical, if we only substitute in the latter $\mathrm{k}_{\mathrm{a}}$ for $\mathrm{k}_{2}$ and $\frac{\mathrm{k}_{\mathrm{w}}}{\mathrm{k}_{\mathrm{b}}}$ for $\mathrm{k}_{\mathrm{1}}$. As in a previous instance (see page 54) the specific replaceability of an acid constant by $\frac{\mathrm{k}_{\mathrm{w}}}{\mathrm{k}_{\mathrm{a}}}$ is equally valid. Also in respect to the formation of the maximum the $\rho$-curve corresponds to the $\alpha_{1}$-curve. All analytic loci of the $\alpha_{1}$ curve hold good in this curve, if we substitute $\mathrm{k}_{\mathrm{b}}$ for $\mathrm{k}_{2}$ and $\frac{\mathrm{k}_{\mathrm{w}}}{\mathrm{k}_{\mathrm{a}}}$ for $k_{1}$. In the $\alpha_{1}$-curve the width of the maximum depended upon the $\mathrm{k}_{1}: \mathrm{k}_{2}$ ratio, therefore in this case it is determined by the product $k_{\mathrm{a}} \times \mathrm{k}_{\mathrm{b}}$. Figure 8 shows the course of various $\rho$-curves in which the product $\mathrm{k}_{\mathrm{a}} \times \mathrm{k}_{\mathrm{b}}$ has different values. $\mathrm{k}_{\mathrm{a}}$ is assumed to be equal to $k_{b}$ in these curves. When $k_{a} \times k_{b}$ is very small, about $10^{-30}$, then the maximum elevation of the curve spreads over a very wide stretch. When this product is larger, about $10^{-18}$, then this elevation becomes smaller, the maximum point may be readily seen, and its ordinate value is around 1. With a still greater increase 
of $\mathrm{k}_{\mathrm{a}} \times \mathrm{k}_{\mathrm{b}}$ the maximum rises less and less, and the elevation zone becomes narrower. Thus for $\mathrm{k}_{\mathrm{a}} \times \mathrm{k}_{\mathrm{b}}=10^{-14}=\mathrm{k}_{\mathrm{w}}$ the maximum value is around only $1 / 3$. However, such ampholytes and those with a still greater $k_{\mathrm{a}} \times \mathrm{k}_{\mathrm{b}}$ product are not known to exist.

The $\rho$-curves of all electrolytes having the same $\mathrm{k}_{\mathrm{a}} \times \mathrm{k}_{\mathrm{b}}$ value are parallel and are but horizontally shifted, as it were, on a common abscissa, as shown in figure 9. In each of such $\rho$-curves an ascending and a descending arm are differentiated. The former is almost exactly the $\rho$-curve of an acid whose dissociation constant is $k_{\mathrm{a}}$, the latter is an $\alpha$-curve of an acid whose constant is $\frac{k_{w}}{k_{b}}$ i.e., a base whose dissociation constant is $\mathrm{k}_{\mathrm{b}}$. This relation is practically true whenever $k_{\mathrm{a}} \times \mathrm{k}_{\mathrm{b}}$ is very small. It follows then that the $\rho$-curve

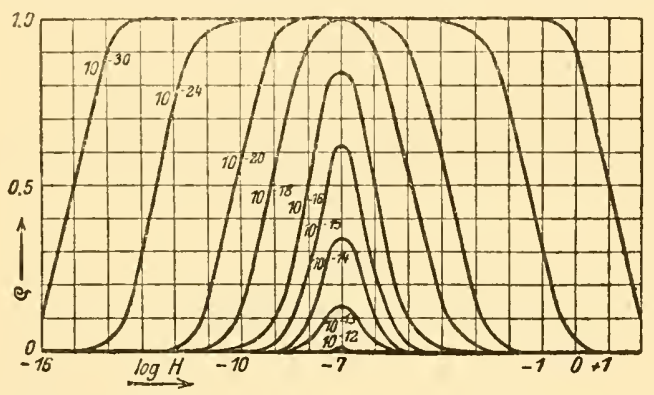

FIG. 8. Dissociation-residue curves for various amphoteric electrolytes of different $k_{a} \times k_{b}$ values. Each curve is marked with its appropriate $k_{a} \times k_{b}$ value. It is assumed throughout that $\mathrm{k}_{\mathrm{a}}=\mathrm{k}_{\mathrm{b}}$.

of an ampholyte may be constructed by considering it in the first place as an acid with the constant $k_{\mathrm{a}}$ and secondly as a base with the constant $k_{b}$ and then by uniting the two respective $\rho$-curves. If, however, $\mathrm{k}_{\mathrm{a}} \times \mathrm{k}_{\mathrm{b}}$ is larger, then this method is not exact, as may be seen from the comparison of the curves in figures 9 and 10 . The deviation is greatest in the vicinity of the maximum, but the abscissa value corresponding to the maximum remains at least unchanged.

The hydrion concentration corresponding to the maximum "of the dissociation-residue curve is designated as the isoelectric point of the ampholyte. This term was originally used to designate the point at which the sign of the charge of a colloid changed. For example, the negatively charged gold sol becomes positively charged 
on the addition of $\mathrm{Al}^{+++}$salts; that concentration of the $\mathrm{Al}^{+++}$ salt at which this change occurred was called the isoelectric point. This conception was introduced by $\mathrm{Hardy}^{36}$ for proteins, when he showed that they are negatively charged in alkaline solution and positively in acid solution. According to Hardy, although it was not specifically emphasized, the isoelectric point of protein was

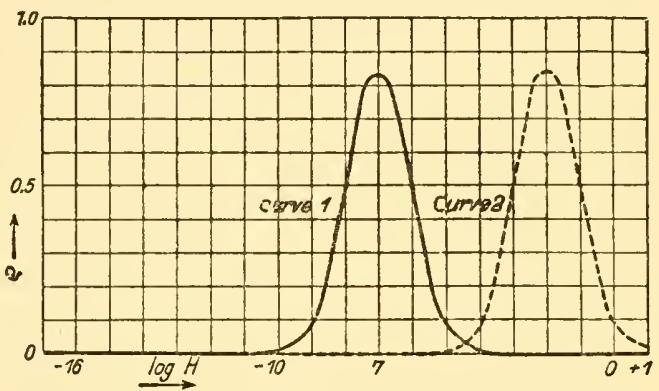

Fig. 9. Dissociation-residue curves for two ampholytes in each of which $\mathrm{ka} \times \mathrm{kb}_{\mathrm{b}}=10^{-16}$, but curve 1 is for the ease where $\mathrm{k}_{\mathrm{a}}=\mathrm{k}_{\mathrm{b}}=10^{-8}$, while curve 2 (dotted) is for the case where $\mathrm{k}_{\mathrm{a}}=10^{-3}$ and $\mathrm{k}_{\mathrm{b}}=10^{-13}$.

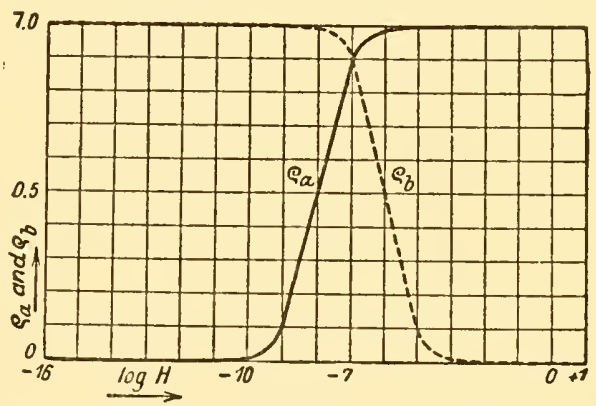

FIg. 10. The dissociation-residue curve $\left(\rho_{\mathrm{a}}\right)$ of an acid whose $k_{a}=10^{-6}$ also of a base whose $\mathrm{k}_{\mathrm{b}}=10^{-6}$ likewise. If curve $\rho_{\mathrm{a}}$ is followed to its intersection with $\rho_{\mathrm{b}}$ and then $\rho_{\mathrm{b}}$ is followed downward, a curve is obtained which is almost identical with curve 1 in figure 9 .

equal to the reaction of neutrality. The definition of the isoelectric point of an ampholyte as the maximum of the $\rho$-curve as given above was developed by Michaelis in $1910 . .^{35}$

The maximum of the $\rho$-curve can also be analytically defined.

${ }^{36}$ W. B. Hardy, Journ. of Physiol. 33, 251 (1905). 
It is easier to calculate it as the minimum of the inverse, $1 / \rho$, function as follows:

$$
\frac{1}{\rho}=1+\frac{k_{a}}{\left[\mathrm{H}^{+}\right]}+\frac{k_{\mathrm{b}}}{k_{\mathrm{w}}} \cdot\left[\mathrm{H}^{+}\right]
$$

and its derivative in respect to $\left[\mathrm{H}^{+}\right]$is:

$$
\frac{\mathrm{d}^{1}}{\mathrm{~d}\left[\mathrm{H}^{+}\right]}=-\frac{\mathrm{k}_{\mathrm{a}}}{\left[\mathrm{H}^{+}\right]^{2}}+\frac{\mathrm{k}_{\mathrm{b}}}{\mathrm{k}_{\mathrm{w}}}
$$

by setting this derivative $=0$ we obtain the minimum of $1 / \rho$ or the maximum of $\rho$, which is

$$
\left[\mathrm{H}^{+}\right] \text {for } \rho \text {-max. }=\sqrt{\frac{\mathrm{k}_{\mathrm{a}}}{\mathrm{k}_{\mathrm{b}}} k_{\mathrm{w}}}
$$

The isoelectric point $\mathrm{I}$ is, therefore, that $\left[\mathrm{H}^{+}\right]$which is determined by the equation

$$
I=\sqrt{\frac{k_{\mathrm{a}}}{k_{\mathrm{b}}} k_{\mathrm{w}}}
$$

It is characterized by the following properties:

1. At its isoelectric point the sum of the anions and cations of an ampholyte in a given concentration is at a minimum.

2. The concentration of the anions of an ampholyte at its isoelectric point is equal to that of the cations.

These conditions may be proved as follows (according to (1) and (2), page 61):

$$
\left[\mathrm{A}^{-}\right]=\frac{k_{\mathrm{a}} \cdot[\mathrm{U}]}{\left[\mathrm{H}^{+}\right]} \text {and }\left[\mathrm{A}^{+}\right]=\frac{\mathrm{k}_{\mathrm{b}} \cdot[\mathrm{U}]}{\left[\mathrm{OH}^{-}\right]}
$$

By substituting for $\left[\mathrm{H}^{+}\right]$its value at the isoelectric point, $\sqrt{\frac{k_{a}}{k_{b}} k_{w}}$, and for $\left[\mathrm{OH}^{-}\right]$the corresponding value

$$
\frac{k_{\mathrm{w}}}{\left[\mathrm{H}^{+}\right]}=\frac{\mathrm{k}_{\mathrm{w}}}{\sqrt{\frac{\mathrm{k}_{\mathrm{a}}}{\mathrm{k}_{\mathrm{b}}} \cdot k_{\mathrm{w}}}}=\sqrt{\frac{\mathrm{k}_{\mathrm{b}}}{\mathrm{k}_{\mathrm{a}}} \cdot k_{\mathrm{w}}},
$$


we obtain

$$
\left[\mathrm{A}^{-}\right]=[\mathrm{U}] \cdot \frac{\mathrm{k}_{\mathrm{a}}}{\sqrt{\frac{\mathrm{k}_{\mathrm{a}}}{\mathrm{k}_{\mathrm{b}}} \cdot \mathrm{k}_{\mathrm{w}}}} \text { and }\left[\mathrm{A}^{+}\right]=[\mathrm{U}] \cdot \frac{\mathrm{k}_{\mathrm{b}}}{\sqrt{\frac{\mathrm{k}_{\mathrm{b}}}{\mathrm{k}_{\mathrm{a}}} \cdot \mathrm{k}_{\mathrm{w}}}}
$$

or

$$
\left[A^{-}\right]=[U] \sqrt{\frac{k_{a} \cdot k_{b}}{k_{w}}} \text { and }\left[A^{+}\right]=[U] \sqrt{\frac{k_{a} \cdot k_{b}}{k_{w}}}
$$

Therefore

$$
\left[\mathrm{A}^{-}\right]=\left[\mathrm{A}^{+}\right]
$$

In the above derivations it is assumed that the electrically uncharged part of the ampholyte is present in one chemical modification only. But the following case is also conceivable. The ampholyte may exist in two forms, $A_{1}$ and $A_{2}$ (such as lactam and lactim forms), so that the cations have only that configuration which corresponds to $A_{1}$ and the anions only that which correspond to $A_{2}$.

The idea would suggest itself then that in equation (1), page 61 , $U_{1}$ should be substituted for $U$, and in (2) $U_{2}$ for $U$, where $U_{1}$ is the concentration of the undissociated $A_{1}$ form and $U_{2}$ that of the undissociated $\mathrm{A}_{2}$ form. The author stated this idea first ${ }^{37}$ and subsequently it was developed by Eckweiller, Noyes and Falk. ${ }^{38}$ These latter authors modified the equation for the isoelectric point in the manner shown

$$
I=\sqrt{\frac{k_{\mathrm{a}}}{\mathrm{k}_{\mathrm{b}}} \cdot \mathrm{k}_{\mathrm{w}} \cdot \frac{\left[\mathrm{A}^{+}\right]}{\left[\mathrm{A}_{1}\right]} \cdot \frac{\left[\mathrm{A}_{2}\right]}{\left[\mathrm{A}_{2}-\right]}}
$$

in which $A$ and $A_{1}$ are the two tautomeric modifications of the ampholyte. This newer interpretation is, however, not quite correct. For in our experiments we determined not the equilibrium between the ampholyte ions and of any tautomeric form of the uncharged ampholyte molecule, but only the equilibrium between the ions and the undissociated ampholyte molecules in toto. Or, in other words, our experimentally determined values of $k_{a}$ and $k_{b}$

${ }^{37}$ Michaelis and Davidsohn, Biochem. Zeitschr. 30, 143 (1910); see p. 149, concerning theobromin.

${ }^{38}$ H. Eckweiller, H. M. Noyes and K. G. Falk, Journ. of Gen. Physiol. 3, 291 (1921). 
denote, as always, the apparent dissociation constants. 'Thus our original equation remains correct, and the contingent deviations from the directly determined isoelectrie point and from that calculated from the dissociation constants will have to be explained in some other way.

\section{Latest developments in the theory of ampholyte drssociation}

The most marked advance in the theory of dissociation is based upon a contribution by Bjerrum, ${ }^{38 a}$ and its important forerunner being an article by Adams. ${ }^{38 b}$ Accordingly it is no longer correct to state the ampholyte ions (Zwitter-Ionen) are present only in extremely small amounts. For the aliphatic amino-acids it seems to hold true that in their "undissociated" state they are present almost entirely as amphoteric ions ${ }^{+} \mathrm{NH}_{3}-\mathrm{R}-\mathrm{COO}^{-}$, while the amount of the molecular species $\mathrm{NH}_{2}-\mathrm{R}-\mathrm{COOH}$ must be considered as being negligible. Isoelectric glycocoll is to a certain extent an inner salt between the $\mathrm{NH}_{2-}$ and the $\mathrm{COOH}$-groups which is completely dissociated, in analogy with ammonium acetate consisting in solution entirely of $\mathrm{NH}_{4}{ }^{+}$and $\mathrm{CH}_{3} \mathrm{COO}^{-}$-ions. Upon the addition of acid to a solution of glycocoll, the ionization of the $\mathrm{R}-$ $\mathrm{COO}^{-}$-group is suppressed, and there are formed $+\mathrm{NH}_{3} \mathrm{CH}_{2} \mathrm{COOH}$ ions (just as with ammonium acetate $\mathrm{NH}_{4}{ }^{+}+\mathrm{CH}_{3} \mathrm{COOH}$ would be formed). Upon adding alkali, the ionization of the ammoniumgroup is suppressed, and the formation of $\mathrm{NH}_{2}-\mathrm{CH}_{2}-\mathrm{COO}^{-}$-ions occur (analogously to the reaction of ammonium acetate + alkali $\left.\rightarrow \mathrm{NH}_{3}+\mathrm{CH}_{3} \mathrm{COO}^{-}\right)$.

$\mathrm{k}_{\mathrm{a}}$, hitherto designated as the acid dissociation constant, relates to a process involving the ammonium group, and $k_{b}$, hitherto designated as the basic dissociation constant, relates to a process involving the COO-group. The following method of presentation ${ }^{38 c}$ appears to be the simplest:

We shall consider as the undissociated form the molecular species $+\mathrm{NH}_{3}-\mathrm{CH}_{2}-\mathrm{COOH}$, on the grounds that this ion contains the greatest number of atoms, and because all other forms of glycocoll may be conceived to arise by the process of splitting off $\mathrm{H}$-ions from

38a N. Bjerrum, Zts. physik. Chem. 104, 147 (1923).

38b E. Q. Adams, J. Amer. Chem. Soc., 38, 1503 (1906).

$38 \mathrm{c}$ L. Michaelis and M. Mizutani, Zts. physik. Chem. 116, 152 (1925). 
this molecular species. Hence in such a case we may consider an ion as the undissociated molecule, whereas in the past we have been accustomed to consider the electroneutral molecule as the undissociated form. This ion then dissociates, similarly to a dibasic acid, in the following way:

1st Stage:

$$
{ }^{+} \mathrm{NH}_{3}-\mathrm{R}-\mathrm{COOH} \rightarrow{ }^{+} \mathrm{NH}_{3}-\mathrm{R}-\mathrm{COO}^{-}+\mathrm{H}^{+}
$$

2nd Stage:

$$
{ }^{+} \mathrm{NH}_{3}-\mathrm{R}-\mathrm{COO}^{-} \rightarrow \mathrm{NH}_{2}-\mathrm{R}-\mathrm{COO}^{-}+\mathrm{H}^{+}
$$

The equilibrium constants are, for the first stage:

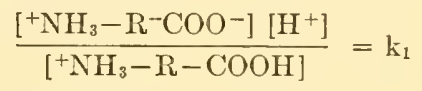

and for the second stage:

$$
\frac{\left[\mathrm{NH}_{2}-\mathrm{R}-\mathrm{COO}^{-}\right]\left[\mathrm{H}^{+}\right]}{\left[\mathrm{NH}_{3}-\mathrm{R}-\mathrm{COO}^{-}\right]}=\mathrm{k}_{2}
$$

The relation of $k_{1}$ and $k_{2}$ to the hitherto employed (and still widely used and for most practical purposes usable) values $k_{a}$ and $k_{b}$ is:

$$
\begin{aligned}
& \mathrm{k}_{a}=\mathrm{k}_{2} \\
& \mathrm{k}_{b}=\frac{\mathrm{k}_{\mathrm{w}}}{\mathrm{k}_{1}}
\end{aligned}
$$

For the aromatic amino acids (such as aminobenzoic acid) this conception does not apply so well. In these the molecular species $\mathrm{NH}_{2}-\mathrm{R}-\mathrm{COOH}$ may actually exist in measurable concentrations along with the ampholyte ion $+\mathrm{NH}_{3}-\mathrm{R}-\mathrm{COO}^{-}$. As for the amphoteric electrolytes with weaker acidogenic radicals, such as aminophenol, on the other hand, the older conception is the more correct one.

Glycocoll is a stronger acid than acetic acid and a stronger base than ammonia. Its inner salt (the ampholyte-ion) is therefore extremely little dissociated hydrolytically which corresponds to stating that there are very few molecules in the form $\mathrm{NH}_{2}-\mathrm{CH}_{2}-$ $\mathrm{COOH}$.

It is very probable that also the proteins in the isoelectric state exist in the ampholyte-ion form. The amphoteric nature of such ions 
manifests itself in their ability to add $\mathrm{H}_{-}$-ions (to the $\mathrm{COO}^{-}$group) as well as to split eff $\mathrm{H}$-ions (from the $\mathrm{NH}_{3}{ }^{+}$-group).

The experimentally observed fact described in the text to the effect that the product $k_{\mathrm{a}} \times \mathrm{k}_{\mathrm{b}}$ of any of the known ampholytes has never been found to be equal to or greater than $\mathrm{k}_{\mathrm{w}}$, has been recently shown by Bjerrum $^{38 a}$ to be a necessary consequence of the above developed conception.

\section{The determination of the isoelectric point}

The isoelectric point of the ordinary ampholytes (the colloidal ampholytes will be dealt with later) may be determined by a variety of methods.

1. The first method is especially applicable to easily soluble ampholytes and is based on the following principle. When acid is added to a dilute buffer solution, i.e., to a solution of a definite $\left[\mathrm{H}^{+}\right]$but of considerable buffer value, the $\left[\mathrm{H}^{+}\right]$rises, and on the addition of a base to the same solution the $\left[\mathrm{H}^{+}\right]$falls. Let us designate again that $\left[\mathrm{H}^{+}\right]$which corresponds to the isoelectric point of an ampholyte by the symbol I. Then the ampholyte in solution will behave as an acid as long as $\left[\mathrm{H}^{+}\right]<\mathrm{I}$, and as a base when $\left[\mathrm{H}^{+}\right]>\mathrm{I}$. In the first case, if added in sufficient concentration, it will raise the $\left[\mathrm{H}^{+}\right]$of the buffer solution, and in the second case it will diminish it. Only at its isoelectric point does the ampholyte remain without any effect upon the buffer, for then it behaves practically as a non-electrolyte; or stated more exactly, because the very few ions yielded by it consist of equal numbers of anions and cations. The number of $\mathrm{H}$-ions cannot, therefore, be increased in the buffer solution on the addition of the isoelectric ampholyte, because there are no corresponding number of negative ions to maintain the electro-neutrality, and for a similar reason the $\left[\mathrm{H}^{+}\right]$ cannot be diminished. The following experiment ${ }^{39}$ is quoted in corroboration of this theory: A constant amount of an aqueous solution of phenylalanine was added to each of a series of buffer solutions consisting of a constant small amount of sodium acetate and increasing amounts of acetic acid. The $\mathrm{pH}$ of these solutions, determined electrometrically, is given in table 13 .

The same could be demonstrated for glycocoll. Its isoelectric

${ }^{39}$ L. Michaelis, Biochem. Zeitschr. 47, 250 (1912). 
point was calculated at $\mathrm{pH} 6.09$ and was found by the above described method to be between $\mathrm{pH} 6.3$ and 5.S.

2. With difficultly soluble ampholytes the following method 40 may be used which is based upon the principle that the undissociated portion of an ampholyte is less soluble than its ionized portion. An equal ample amount of an ampholyte is added to each of a series of hot acetate buffer solutions and the appearance of crystallization is observed. A suitable ampholyte for this purpose is $m$ aminobenzoic acid. In the following table the original $\mathrm{pH}$ of the

TABLE 13

\begin{tabular}{|c|c|c|c|}
\hline & $\begin{array}{c}\mathrm{pH} \text { before } \\
\text { addition of } \\
\text { phenylalanine }\end{array}$ & $\begin{array}{c}\mathrm{pH} \text { after } \\
\text { addition of } \\
\text { phenylalanine }\end{array}$ & $\begin{array}{c}\text { Difference in } \\
\text { pH }\end{array}$ \\
\hline Phenylalanine as base ........... & $\begin{array}{l}3.75 \\
4.10 \\
4.27\end{array}$ & $\begin{array}{l}4.01 \\
4.22 \\
4.43\end{array}$ & $\begin{array}{r}+0.28 \\
+0.12 \\
+0.16\end{array}$ \\
\hline \multicolumn{4}{|c|}{ Isoelectric point calculated at $\mathrm{pH}=4.48$} \\
\hline & 4.58 & 4.57 & -0.01 \\
\hline \multirow{5}{*}{ Phenylalanine as acid ............ } & 4.66 & 4.55 & -0.11 \\
\hline & 5.07 & 4.78 & -0.29 \\
\hline & 5.26 & 4.74 & -0.52 \\
\hline & 5.45 & 4.72 & -0.73 \\
\hline & 5.73 & 4.77 & -0.96 \\
\hline
\end{tabular}

buffer solutions is given in the upper and the observed extent of crystallization in the lower line:

$\begin{array}{lccccccccc}\text { pH......... } & 5.4 & 5.1 & 4.8 & 4.5 & 4.2 & 3.9 & 3.6 & 3.3 & 3.1 \\ \text { Crystallization: } & 0 & 0 & + & +++ & ++++ & +++ & 0 & 0 & 0\end{array}$

It appears then that the crystallization maximum is in the close vicinity of $\mathrm{pH} 4.2$, while the isoelectric point, as calculated from the dissociation constants found in the literature, is at $\mathrm{pH}$ 4.1. The crystallization optimum of $p$-aminobenzoic acid may be determined with equal definiteness. The relative sharpness of definition of such optima depends upon the value of the product $\mathrm{k}_{\mathrm{a}} \times \mathrm{k}_{\mathrm{b}}$, just as the sharpness of the maximum of the dissociation-residue curve

${ }^{40}$ L. Michaelis and H. Davidsohn, Biochem. Zeitschr. 30, 140 (1910). 
(see figure 8) depends upon the same value. The sharply defined minima of the aminobenzoic acids corresponds to

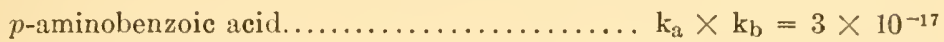

$m$-aminobenzoic acid................ ka $\times \mathrm{k}_{\mathrm{b}}=2 \times 10^{-16}$

On the other hand, it has long been known that such ampholytes as tyrosine and arsenious acid have quite poorly defined (flat) maxima. These are soluble in strong acids and bases but quite insoluble in the wide intermediate range of reaction. No sharply defined optimum in relation to the $\mathrm{pH}$ of solution is to be found in this case, and the small order of the corresponding values of the dissociation constants products is to be noted:

Tyrosine................................

Arsenious acid....................... $\mathrm{k}_{\mathrm{a}} \times \mathrm{k}_{\mathrm{b}}=6 \times 10^{-24}$

Employing the above method Michaelis and Davidsohn obtained the following values:

\begin{tabular}{|c|c|c|c|c|}
\hline & $\mathrm{k}_{\mathrm{a}}$ & $\mathrm{k}_{\mathbf{b}}$ & I calc. & I found \\
\hline o-Aminobenzoic acid. & $1.21 \times 10^{-5}$ & $2.33 \times 10^{-12}$ & $1.7 \times 10^{-4}$ & $1.6 \times 10^{-4}$ \\
\hline m-Aminobenzoic acid & $1.63 \times 10^{-5}$ & $1.22 \times 10^{-11}$ & $8.8 \times 10^{-5}$ & $6.3 \times 10^{-5}$ \\
\hline Aspartic acid....... & $1.5 \times 10^{-4}$ & $1.2 \times 10^{-12}$ & $8.7 \times 10^{-4}$ & $9.3 \times 10^{-4}$ \\
\hline
\end{tabular}

It may be of interest to compare the results obtained by various investigators, partly for varying temperatures (table 15).

The agreement among the above figures is quite good, especially when they are reduced to the same temperature. The uncertainties which existed formerly in regard to some of the figures were due to the fact that the influences of salt concentration had not been properly understood and corrected for. With due consideration for some of the newer principles the lack of agreement among some of the older figures will disappear. This applies to all dissociation constants, and not only to those of the ampholytes.

Of all of the above ampholytes only arginine, lysine and histidine have (in their second stage of dissociation) larger $k_{b}$ values than those of $k_{\mathrm{a}}$; the isoelectric point of only these ampholytes is at an alkaline reaction. 
TABLE 14

Table of dissociation constants of amphoteric electrolytes at $25^{\circ}$

The isoelectric point values in this table were calculated from the equation $\mathrm{I}=\sqrt{\frac{\mathrm{k}_{\mathrm{a}} \mathrm{k}_{\mathrm{w}}}{\mathrm{k}_{\mathrm{b}}}}$, on the assumption that $\mathrm{k}_{w}=10^{-14}$.

\begin{tabular}{|c|c|c|c|c|c|c|}
\hline & & $\mathrm{k}_{\mathrm{a}}$ & & $\mathrm{k}_{\mathrm{b}}$ & Observer & Isoelectric point \\
\hline $\begin{array}{l}\text { Arginine, } 2 \text { nd } \\
\text { stage....... }\end{array}$ & & - & 2.2 & $\times 10^{-12}$ & Kanitz & \\
\hline $\begin{array}{l}\text { Arginine, 1st } \\
\text { stage....... }\end{array}$ & $<1.1$ & $\times 10^{-14}$ & 1.0 & $\times 10^{-7}$ & Kanitz & ca. 3 \\
\hline $\begin{array}{l}\text { Lysine, } 2 \mathrm{nd} \\
\text { stage....... }\end{array}$ & & - & 1.1 & $\times 10^{-12}$ & Kanitz & ca. 3 \\
\hline $\begin{array}{l}\text { I,ysine, } 1 \text { st } \\
\text { stage...... }\end{array}$ & 1.0 & $\times 10^{-12}$ & $<1.1$ & $\times 10^{-7}$ & Kanitz & ca. $3 \times 10^{-10}$ \\
\hline Leucine....... & 1.8 & $\times 10^{-10}$ & 2.3 & $\times 10^{-12}$ & Winkelblech & $8.8 \times 10^{-7}$ \\
\hline coll..... & 1.8 & $\times 10^{-10}$ & 2.7 & $\times 1$ & Winke & $8.2 \times 10^{-7}$ \\
\hline $\begin{array}{l}\text { Alanine....... } \\
\text { Histidine, 2nd }\end{array}$ & 1.9 & $\times 10^{-10}$ & 5.1 & $\times 10^{-12}$ & Winkelblech & $6.1 \times 10^{-7}$ \\
\hline we, 1st & & - & 5.0 & $\times 10^{-13}$ & Kanitz & \\
\hline & 2.2 & $\times 10^{-9}$ & 5.7 & $\times 10^{-9}$ & Kanitz & $6.2 \times 10^{-8}$ \\
\hline Phenylalanine & 2.5 & $\times 10^{-9}$ & 1.3 & $\times 10$ & Ka & $4.4 \times 10^{-6}$ \\
\hline$\cdots \cdots$ & 4.0 & $\times 10$ & 2.6 & $\times 1$ & $\mathrm{Ka}$ & $3.9 \times 10^{-6}$ \\
\hline Leucylglycine & 1.5 & $\times 10^{-8}$ & 3.0 & $\times 1$ & $\mathrm{Eu}$ & $2.2 \times 10^{-6}$ \\
\hline cine & 1.8 & $\times 10$ & 2.0 & $\times 10$ & $\mathrm{Eu}$ & $3.0 \times 10^{-6}$ \\
\hline Iglycine & 1.8 & $\times 10^{-8}$ & 2.0 & $\times 1$ & En & \\
\hline Aspartic acid. & 1.5 & $\times 10^{-4}$ & 1.2 & $\times 10^{-12}$ & $\begin{array}{l}\text { Winkelblech, } \\
\text { Lundén }\end{array}$ & $1.1 \times 10^{-3}$ \\
\hline $\begin{array}{l}\text { Arsenious } \\
\text { acid.......... } \\
\text { o-Aminoben- }\end{array}$ & 6 & $\times 10^{-10}$ & & $\times 10^{-14}$ & Wood & $2.4 \times 10^{-5}$ \\
\hline $\begin{array}{l}\text { zoic acid... } \\
\text {-Aminoben. }\end{array}$ & 1.06 & $\times 10^{-5}$ & 1.37 & $\times 10^{-12}$ & Lundén & $2.8 \times 10^{-4}$ \\
\hline zoic acid... & 1.21 & $\times 10^{-5}$ & 2.33 & $\times 10^{-11}$ & $\begin{array}{l}\text { Winkelbech, } \\
\text { Walker }\end{array}$ & $7.2 \times 10^{-3}$ \\
\hline $\begin{array}{l}\text { m-Aminoben- } \\
\text { zoic acid... }\end{array}$ & 1.6 & $\times 10^{-5}$ & 1.20 & $\times 10^{-12}$ & $\begin{array}{l}\text { Winkelbech, } \\
\text { Walker }\end{array}$ & $1.1 \times 10^{-4}$ \\
\hline
\end{tabular}


TABLE 15

\begin{tabular}{|c|c|c|c|}
\hline & & Temperature & Observer" \\
\hline \multirow[t]{3}{*}{ Glycocoll, $\mathrm{k}_{\mathrm{a}} \ldots \ldots \ldots \ldots$} & $1.8 \times 10^{-10}$ & $25^{\circ}$ & Winkelblech \\
\hline & $1.2 \times 10^{-10}$ & $17.5^{\circ}$ & Michaelis and Rona \\
\hline & $1.05 \times 10^{-10}$ & $18^{\circ}$ & Dernby \\
\hline \multirow[t]{3}{*}{ Glycocoll, $\mathrm{k}_{\mathrm{b}} \ldots \ldots \ldots \ldots$} & $2.7 \times 10^{-12}$ & $25^{\circ}$ & Winkelblech \\
\hline & $1.93 \times 10^{-12}$ & $17.5^{\circ}$ & Michaelis and Rona \\
\hline & $1.7 \times 10^{-12}$ & $18^{\circ}$ & Dernby \\
\hline \multirow[t]{2}{*}{ Glycylglycocoll, $\mathrm{k}_{\mathrm{a}} \ldots . .$. . } & $1.8 \times 10^{-8}$ & $25^{\circ}$ & Euler \\
\hline & $3.3 \times 10^{-9}$ & $18^{\circ}$ & Dernby \\
\hline \multirow[t]{2}{*}{ Glycylglycocoll, $\mathrm{k}_{\mathrm{b}} \ldots \ldots$... } & $2 \times 10^{-11}$ & $25^{\circ}$ & Euler \\
\hline & $0.95 \times 10^{-11}$ & $18^{\circ}$ & Dernby \\
\hline
\end{tabular}

* Winkelblech, Zeitschr. f. physikal. Chem., 46, 546 (1901).

Michaelis and Rona, Biochem. Zeitschr. 49, 248 (1913).

Dernby, Cpt. rend. des travaux du lab. de Carlsberg. 11, 265 (1916).

H. Euler, Hofmeisters Beitr. 7, 1 (1906).

\section{Amphoteric ions}

Bredig pointed out that one of the ionization forms of an ampholyte of the general type $\mathrm{NH}_{2}-\mathrm{R}-\mathrm{COO}$ must be an amphoteric ion ("Zwitter-Ion") which is positively as well as negatively charged, such as ${ }^{+} \mathrm{NH}_{3}-\mathrm{R}-\mathrm{COO}^{-}$. This molecular species may also be conceived of as an inner salt formation arising between the COOHand the $\mathrm{NH}_{2}$-groups. Thus, for instance,

$$
\mathrm{CH}_{3} \mathrm{COOH}+\mathrm{NH}_{3} \rightleftharpoons \mathrm{CH}_{3} \mathrm{COO}^{-}+\mathrm{NH}_{4}{ }^{+}
$$

In the same manner we may assume that the salt arising from the internal salt-formation of the amino-acid dissociates electrolytically and produces the ion $+\mathrm{NH}_{3}-\mathrm{R}-\mathrm{COO}^{-}$. It is not possible to demonstrate the existence of this molecular species by the usual method of demonstrating ions, i.e., the migration in one direction in an electric current, for this amphoteric ion is electrically neutral. Besides, it is undoubtedly present in extremely small amounts. Furthermore, an amino-acid being the salt of so weak an acid and so weak a base always hydrolyses to a great extent, and the product of hydrolysis of the amphoteric ion is the usual form of the aminoacid. Just as the hydrolysis product of ammonium acetate is ammonia and acetic acid, or,

$$
\mathrm{NH}_{4}{ }^{+}+\mathrm{CH}_{3} \mathrm{COO}^{-} \rightleftharpoons \mathrm{NH}_{3}+\mathrm{CH}_{3} \mathrm{COOH}
$$


or, as it is customarily written

$$
\mathrm{CH}_{3} \mathrm{COO} \mathrm{NH}_{4}+\mathrm{H}_{2} \mathrm{O} \rightleftharpoons \mathrm{CH}_{3} \mathrm{COOII}+\mathrm{NH}_{4} \mathrm{OH}
$$

the amphoteric ion likewise yields by hydrolysis the ordinary aminoacid molecule:

$$
+\mathrm{NH}_{3}-\mathrm{R}-\mathrm{COO}-\rightleftharpoons \mathrm{NH}_{2}-\mathrm{R}-\mathrm{COOH}
$$

Since one mol of amino-acid yields one mol of amphoteric ion, it follows that for a given amino-acid the concentration of the amphoteric ion must be an equivalent fraction of the concentration of the undissociated amino-acid. There is no direct method up to the present time of demonstrating concretely the existence of the amphoteric ions. Because of their very small concentration they are not capable of disturbing to a measurable extent the equilibrium among the other known dissociation products of amino-acids.

\section{The hydrogen ion concentration of a pure solution of ampholyte}

It is apparent that a pure aqueous solution of an ampholyte must have a neutral reaction whenever $k_{a}=k_{b}$, that it must be acid in reaction when $k_{\mathrm{a}}>\mathrm{k}_{\mathrm{b}}$ and alkaline when $\mathrm{k}_{\mathrm{a}}<\mathrm{k}_{\mathrm{b}}$. The exact calculation of the hydrion concentration, however, is not a simple process. Sørensen ${ }^{41}$ adopted the following method of calculation. Let $\mathrm{A}$ represent the total concentration of the amino-acid and $\mathrm{A}^{+}$, $\mathrm{A}^{-}$and $\mathrm{U}$ the concentrations of its cations, anions and undissociated portion respectively. It then follows

$$
\begin{aligned}
\mathrm{A}^{+}+\mathrm{A}^{-}+\mathrm{U} & =\mathrm{A} \\
\mathrm{A}^{+} \times \mathrm{H}^{+} & =\mathrm{k} \times \mathrm{U} \\
\mathrm{A}^{+} \times \mathrm{OH}^{-} & =\mathrm{k} \times \mathrm{U} \\
\mathrm{H}^{+} \times \mathrm{OH}^{-} & =\mathrm{k}_{\mathrm{W}}
\end{aligned}
$$

and furthermore, in accordance with the law of electroneutrality,

$$
\mathrm{A}^{+}+\mathrm{H}^{+}=\mathrm{A}^{-}+\mathrm{OH}^{-}
$$

If the values of $\mathrm{A}, \mathrm{k}_{\mathrm{a}}$ and $\mathrm{k}_{\mathrm{b}}$ be given, then the five unknown values of $\mathrm{U}, \mathrm{A}^{+}, \mathrm{A}^{-},\left[\mathrm{H}^{+}\right]$and $\left[\mathrm{OH}^{-}\right]$may be calculated by solving

${ }^{41}$ S. P. L. Sörensen, Ergebn. d. Physiol. 12, 495 ff. (1912). 
the five above equations. In carrying out the calculation for $\left[\mathrm{H}^{+}\right]$, we get from (I), (II) and (III)

$$
\frac{\mathrm{k}_{\mathrm{a}} \cdot \mathrm{U}}{\mathrm{H}^{+}}+\frac{\mathrm{k}_{\mathrm{b}} \cdot \mathrm{U}}{\mathrm{OH}^{-}}+\mathrm{U}=\mathrm{A}
$$

and from (II, III, V):

$$
\mathrm{U}=\frac{\mathrm{H}^{+}-\mathrm{OH}^{-}}{\frac{\mathrm{k}_{\mathrm{a}}}{\mathrm{H}^{+}}-\frac{\mathrm{k}_{\mathrm{b}}}{\mathrm{OH}^{-}}}
$$

By substituting this value of $U$ in (VI) we obtain:

$$
\frac{\mathrm{k}_{\mathrm{a}}}{\mathrm{H}^{+}}+\frac{\mathrm{k}_{\mathrm{b}}}{\mathrm{OH}^{-}}+1=\frac{\mathrm{A} \cdot\left(\frac{\mathrm{k}_{\mathrm{a}}}{\mathrm{H}^{+}}-\frac{\mathrm{k}_{\mathrm{b}}}{\mathrm{OH}^{-}}\right)}{\mathrm{H}^{+}-\mathrm{OH}^{-}}
$$

in which we can further substitute $\mathrm{k}_{\mathrm{w}} /\left[\mathrm{H}^{+}\right]$for $\left[\mathrm{OH}^{-}\right]$. This gives an equation of the fourth order for $\left[\mathrm{H}^{+}\right]$as given below, where $\mathrm{x}$ stands for $\left[\mathrm{H}^{+}\right]$

$$
\begin{aligned}
x^{4}+x^{3}\left(\frac{k_{w}}{k_{b}}+A\right)+x^{2} \frac{k_{w}}{k_{b}}\left(k a-k_{b}\right) & -x \frac{k_{w}}{k_{b}}\left(k_{a} A+k_{w}\right) \\
& -\frac{k_{w}}{k_{b}} \cdot k_{a} \cdot k_{w}=0
\end{aligned}
$$

The general solution of this equation is difficult. It can have only one real positive root, because it contains only one sign change, irrespective of the sign of the coefficient of $\mathrm{x}^{2}$ (Descartes' law). The practical solution rests upon the permissibility of disregarding the first $\left(\mathrm{x}^{4}\right)$ or the last member of the equation, depending upon the numerical relationships of the individual constants. Furthermore equation (VII) may be factored in the following way (this may be tested by simply multiplying out):

$$
\begin{aligned}
& \left(x^{2}-k_{w}\right) \times\left(x^{2}+x \frac{k_{w}}{k_{b}}+x \cdot A+\frac{k_{a}}{k_{b}}\right) k_{w}-x \times A \times k_{w}\left(\frac{k_{a}}{k_{b}}-1\right)=0 \\
& \left.\begin{array}{llll}
\mathrm{Q} & \mathrm{R} & -\mathrm{S} & =0
\end{array}\right\}
\end{aligned}
$$

By letting $\mathrm{S}=0$, i.e., by making $\mathrm{k}_{\mathrm{a}}=\mathrm{k}_{\mathrm{b}}$, equation (VIII) is satisfied when either $\mathrm{Q}$ or $\mathrm{R}=0$. When $\mathrm{R}=0$, no real positive 
root for $\mathrm{x}$ is obtained. On the other hand, when $\mathrm{Q}=0$, we obtain $\mathrm{x}=\sqrt{\mathrm{k}_{\mathrm{w}}}$, which means that a solution of an ampholyte in which $k_{a}=k_{b}$, has a neutral reaction in all concentrations.

Now let us write (VIII) in the form

$$
\mathrm{Q}=\frac{\mathrm{S}}{\mathrm{R}}
$$

or,

$$
\mathrm{x}^{2}=\mathrm{k}_{\mathrm{w}}+\frac{\mathrm{S}}{\mathrm{R}}
$$

Since $R$ is always positive, then $x^{2}>k_{w}$, when $S$ is positive; and $x^{2}<k_{w}$ when $S$ is negative. But $S$ is positive or negative when $k_{\mathrm{a}}>\mathrm{k}_{\mathrm{b}}$ or $\mathrm{k}_{\mathrm{a}}<\mathrm{k}_{\mathrm{b}}$ respectively. In other words: when $k_{a}>k_{b}$ the solution of the ampholyte is always acid, and when $k_{a}$ $<k_{b}$ it is always alkaline.

Equation (VII) solved for A becomes

$$
A=\frac{\left(x^{2}-k_{w}\right)\left(x^{2}+x \frac{k_{w}}{k_{b}}+k_{w} \cdot \frac{k_{a}}{k_{b}}\right)}{x\left(k_{w} \cdot \frac{k_{a}}{k_{b}}-x^{2}\right)}
$$

Now, when $k_{\mathrm{a}}>\mathrm{k}_{\mathrm{b}}$, and, therefore, according to the above reasoning, also $\mathrm{x}^{2}>\mathrm{k}_{\mathrm{w}}$, then both factors of the numerator are positive, consequently the denominator must also be positive, i.e., we must have

$$
x<\sqrt{\frac{k_{w} k_{a}}{k_{b}}}
$$

which denotes that $\mathrm{x}$ is smaller than the $\left[\mathrm{H}^{+}\right]$at the isoelectric point, or, that the $\left[\mathrm{H}^{+}\right]$of the solution must lie between the neutral reaction and that of the isoelectric point. Indeed, with decreasing concentrations of the electrolyte $\mathrm{x}$ approaches the neutral reaction, while it approaches the isoelectric point with increasing concentrations.

By writing equation (IX) in the form

$$
A=\frac{\left(1-\frac{\sqrt{k_{w}}}{x}\right)\left(x+\sqrt{k_{w}}\right)\left(x^{2}+x \frac{k_{w}}{k_{b}}+\frac{k_{w} k_{a}}{k_{b}}\right)}{\frac{k_{w} k_{a}}{k_{b}}-x^{2}}
$$


we perceive that $A$ must increase with increasing values of $x$. For $A=\infty, x$ must have its maximum value, which, according to the last italicized rule above, may only be that of the isoclectric point. In other words, the more concentrated the ampholyte solution the closer does its $\left[\mathrm{H}^{+}\right]$approach that of the isoelectric point of the ampholyte. For $\mathrm{A}=0$ we obtain, on the other hand, the other limiting value, $\mathrm{x}=\sqrt{\mathrm{k}_{\mathrm{w}}}$, or the neutral reaction.

By applying the above outlined general solution of equation (VII) and the rules developed from it, the value of $\mathrm{x}$ may be estimated for any special case. By substituting this estimated value in equation (VII) together with the appropriate values for $k_{a}, k_{b}$ and $\mathrm{k}_{\mathrm{w}}$, it becomes possible to determine whether one or more terms of the expression become extremely small in respect to the other terms, and may then be disregarded. Thus approximate values for $\mathrm{x}$ are obtained, which may be verified by substitution in (VII), and, whenever necessary, corrected by actual trial. By this method Sørensen made the following calculations for pure aqueous solutions of glycocoll, for which $\mathrm{k}_{\mathrm{a}}=1.8 \times 10^{-10}, \mathrm{k}_{\mathrm{b}}=$ $2.7 \times 10^{-12}, \mathrm{k}_{\mathrm{w}}=10^{-14}$ :

Concentration of the solution:

\begin{tabular}{|c|c|c|c|c|c|c|c|c|c|c|}
\hline & 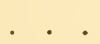 & . & 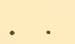 & . & $\infty$ & 1 & $10^{-1}$ & $10^{-2}$ & $10^{-3}$ & $10^{-4}$ \\
\hline & & & & & 3.088 & 6.089 & 6.096 & 6.155 & 6.413 & \\
\hline
\end{tabular}

The isoelectric point is at $\mathrm{pH}=6.088$.

\section{The hydrogen ion concentration and the solubility of weak acids}

This problem takes us back again to the simple electrolytes. It is commonly known that free acids are less soluble than their alkali salts and that bases are less soluble than their chlorides. Since, as was shown above, the relative amounts of free acids and of their ions, as well as of salts, depends upon the hydrion concentration, it follows, therefore, that the hydrion concentration of a solution must have an effect upon the solubility of the electrolytes. Let us next define the concept of solubility. We shall choose for illustrative purposes the solution of a fairly insoluble acid. When water is saturated with solid benzoic acid a system is finally obtained which has the following composition: A sediment of solid benzoic acid which is not electrolytically dissociated to any measurable extent. 
In the supernatant solution we have molecules of undissociated benzoic acid, $\mathrm{H}$-ions and benzoate-ions. (In addition to the $\mathrm{H}$-ions there must also be present $\mathrm{OH}$-ions in accordance with the laws of dissociation. But since these play no part in this consideration they may be ignored). 'Two interpretations may then be given to the expression "solubility of solid benzoic acid." Either it may mean the concentration of those molecules of undissociated benzoic acid which are present in the saturated solution, i.e., the concentration in the saturated supernatant solution of the molecular species of which the sediment is composed. Secondly, the term "solubility" in this case may be regarded as being denoted by the concentration of all the benzoic acid molecules regardless of the form in which they are present in the saturated solution. We shall designate the first as the "partial solubility," or $\lambda$, of benzoic acid and the second as the "total solubility," or $\Lambda$. Furthermore, one may also speak of the "solubility of the benzoate ions." It is apparent that the benzoate ions are much more soluble in water than the free benzoic acid, which is easily demonstrated by the greater solubility of alkali benzoates. It is also occasionally stated that in a pure benzoic acid solution the state of saturation exists only for the undissociated acid, and that, on the other hand, the benzoic acid solution cannot be saturated in respect to the benzoate ions. But this statement is not in accord with the true conception of solubility. Solubility is represented by the concentration reached by a substance in solution, when the solution is in contact with the solid phase. Thus the definition of solubility includes two contiguous phases, and solubility represents the state of equilibrium between the two phases. Without the contact with the solid phase the solubility of a substance cannot be properly defined. Under certain conditions a metastable supersaturated solution may be obtained without the presence of a solid phase. But as soon as a small amount of the solid substance is added to such a supersaturated solution, the excess of the solute separates out until the true state of saturation is reached. Therefore, it cannot be properly stated that a saturated solution of benzoic acid is not saturated in respect to the ions of the acid. For we are dealing here with the definition of the solubility of the undissociated acid alone of which the solid phase is composed. As for the acid anions, the amount of 
these in solution is entirely conditioned by the mass law relationship of concentrations, in the following manner:

$$
\frac{[\text { Acid anions }] \times\left[\mathrm{H}^{+}\right]}{[\text {Undissociated acid }]}=\mathrm{k}
$$

$\mathrm{k}$ is in this case the dissociation constant of the acid. Since the concentration of the undissociated acid is equal to the "partial solubility" of the acid, $\lambda$, therefore, it follows that

$$
[\text { Acid anions }]=\frac{\mathrm{k} \times \lambda}{\left[\mathrm{H}^{+}\right]}
$$

The "total solubility" of the acid, $\Lambda$, is therefore

$$
\Lambda=\lambda+\frac{k \cdot \lambda}{\left[\mathrm{H}^{+}\right]}=\lambda \frac{\left[\mathrm{H}^{+}\right]+k}{\left[\mathrm{H}^{+}\right]}
$$

or, by substituting the values given in (3) on page 52 .

$$
\Lambda=\frac{\lambda}{\rho}
$$

The total solubility, $\Lambda$, is, therefore, dependent upon the $\left[\mathrm{H}^{+}\right]$ of the solution, while $\lambda$, the partial solubility, remains constant (for a given temperature). This calculation is based upon the assumption that the undissociated salt of the acid is not present in solution. In such cases where this condition is not fulfilled a correction may be applied, but we need not consider this point in this general discussion at present.

These relations may be also conceived in other ways. It has been shown that certain solid salts (e.g., silver salts) at higher temperatures conduct the electric current not as metals but as electrolyte solutions to a small but noticeable extent and display material transport of ions. It must be assumed then that at least a very small part of a solid electrolyte dissociates, i.e., it consists of transportable ions. ${ }^{42}$ Thus in a saturated solution of benzoic acid

${ }^{42}$ The present day conception of a crystalline electrolyte consisting only of ions arranged in a space lattice ("Raumgitter") has nothing to do with the above discussion. For the ions arranged in the space lattice are not in free motion; they are ions only insofar as they have the same intra-atomic structure as the ions of a solution in Faraday's sense; but they are not ions insofar as they are not freely movable. 
in water both the solid phase and the liquid phase consist of undissociated acid, H-ions and acid anions. According to the HenryNernst law of distribution each of these three molecular species has its own distribution coefficient in respect to each phase, and the saturated solution should consequently contain these three components distributed between its two phases in concentrations limited by the appropriate coefficients. But this would lead to an unequal concentration of the positive $\mathrm{H}$-ions and of the negative acid ions in solution, which would be impossible because of the electro-static forces arising from such a condition. Instead of this, a condition of equilibrium is established in the system entailing equal concentrations of positive and negative ions in solution and the formation of an electric potential difference at the boundary of the liquid and solid phases (this point to be discussed later). This conception is based upon the distribution coefficient of only one of the molecular species, the undissociated acid, which determines the "partial solubility" of the benzoic acid, and it is also based upon the mass action law which regulates the concentrations of the other molecular species in solution so as to maintain equilibrium.

A biologically important example of the relation of the hydrion concentration to the solubility of an acid is furnished by the case of uric acid. ${ }^{43}$ Unfortunately this problem is not yet completely solved. The investigation of this question has hitherto taken a tortuous path because of a lack of a clear conception of the interdependence of solubility and hydrion concentration. We shall attempt to interpret the available data in accord with the above theory. First the partial solubility, $\lambda$, of uric acid must be determined. For this purpose its ionization must be completely suppressed by means of the addition of $\mathrm{HCl}$. His and Paul found a solubility in $1.0 \mathrm{~N} \mathrm{HCl}$ at $18^{\circ}$ of one mol in 7137 liters, or, $\lambda=$ $1.401 \times 10^{-4}$. According to the same authors the constant of dissociation in the first step, $\mathrm{k}_{1}=1.5 \times 10^{-6}$ (according to Gudzent,

${ }^{43}$ Literature: His and Paul, Pharmazeut. Zeit. 1900.-Paul, Zeitschr. f. physiol. Chem. 31, 1 and 64 (1900).-Gudzent, Zeitschr. f. physiol. Chem. 56, $150(1908) ; 60,25,38 ; 63,253,455$ (1909).-Bechhold and Ziegler, Biochem. Zeitschr. 20, 189 (1909); 24, 146 (1910).-Ringer, Zeitschr. f. physiol. Chem. 67, 332 (1910).-Kohler, Zeitschr. f. physiol. Chem. 70, 360; 72, 169; Zeitschr. f. klin. Med. 78, 1.-Lichtwitz, Zeitschr. f. physiol. Chem. 84, 416 (1913).Schade and Boden, Zeitschr. f. physiol. Chem. 83, 347; 86, 416 (1913).-A. Kanitz, Zeitschr. f. physiol. Chem. 116, 96 (1921). 
$\mathrm{k}_{1}=2.3 \times 10^{-8}$ at $\left.37^{\circ}\right)$. Therefore, at any hydrion concentration (as long as it is not so small as to permit the uric acid, which is dibasic, to begin the second dissociation stage) the solubility at $18^{\circ}$ is:

$$
\text { at } 18^{\circ} \Lambda=1.40 \times 10^{-4} \frac{\left(\left[\mathrm{H}^{+}\right]+1.5 \times 10^{-8}\right)}{\left[\mathrm{H}^{+}\right]}
$$

Thus for example at the reaction of the blood, $\left[\mathrm{H}^{+}\right]=4.5 \times 10^{-8}$,

$$
\Lambda=4.77 \times 10^{-3}
$$

which is thirty times the value of $\Lambda$ in $N \mathrm{HCl}$.

A pure saturated solution of uric acid must have at $15^{\circ}$ a hydrion concentration according to (1a) page 40 .

$$
\left[\mathrm{H}^{+}\right]=\sqrt{1.5 \times 10^{-6} \cdot 1.4 \times 10^{-4}}=1.45 \times 10^{-5}
$$

The degree of dissociation of this solution is, according to (2), page 42 .

$$
\alpha=\frac{1.5 \times 10^{-6}}{1.5 \times 10^{-6}+1.45 \times 10^{-5}}=0.094
$$

Paul and His found experimentally the value of $\alpha$ at $18^{\circ}$ to be 0.095, and, moreover, conversely they derived the dissociation constant from the above value of $\alpha$. The verification of the theory is then afforded by the fact that $\lambda$ and $\Lambda$ are calculable from each other. The solubility of uric acid calculated in the above manner did not have the hoped for significance in physiology and pathology because of the following two complications:

The above considerations are valid only for the system in which uric acid is the solid phase. The presence of the alkali salt of the weak acid did not enter into the derivation, for the alkali salts of almost all acids are much more soluble than the acids themselves. But in this respect uric acid forms an exception. The primary sodium urate is quite insoluble by itself, while in the presence of $\mathrm{NaCl}$ it is an unusually insoluble salt. Furthermore, according to Gudzent, the primary salt exists in two modifications, the lactam and the lactim forms, the solubilities of which are different. If, therefore, one of these forms is present as the solid phase, then the solubility of uric acid becomes a very complicated problem; 
especially since the presence of $\mathrm{NaCl}$ depresses enormously the solubility of the sodium urate. Thus we have the remarkable case in which the presence of the alkali-salt, at least in NaCl-containing solutions, diminishes, instead of increasing, the solubility of a weak acid. Recently Kanitz calculated from available data the second dissociation constant of uric acid, and he came to the conclusion that the primary sodium urate in pure solution is markedly hydrolyzed into free uric acid and the secondary sodium salt. Such a solution contains, therefore, free uric acid which must exercise a great influence upon the solubility of the urate. On the other hand, the sodium urate tends to an abnormally great extent to form very stable supersaturated solutions, even in the presence of the urate as the solid phase. Schade considered these supersaturated solutions as colloidal in nature, but Kohler and Gudzent showed this assumption to be improbable by various means (ultrafiltration, compensation dialysis, conductivity).

Schade and Boden showed that uric acid solutions may be easily made to form gels, which however slowly crystallized out. On the other hand, Gudzent demonstrated by means of compensation dialysis that in the blood uric acid is present in true solution. At any rate, Bechold and Ziegler found that they could dissolve large amounts of free uric acid in blood serum, and they designated this condition as the "overfilling" ("Überfüllung") of the blood with uric acid. Evidently in this case the uric acid is present in the blood in the form of supersaturated sodium urate in solution. The whole problem demands extensive reinvestigation, for we have not yet reached an adequate solution.

\section{The relation of hydrion concentration to the solubility of difficultly soluble salts}

The solubility of true neutral salts $\left(\mathrm{NaCl}, \mathrm{KNO}_{3}\right.$, etc.) does not depend upon the hydrion concentration which is the case for the salts of strong bases and weak acids, and reversely, for the salts of strong acids and weak bases. A biologically important instance is furnished by the solubility of calcium carbonate, which was investigated by Rona and Takahashi. ${ }^{44}$

We shall discuss this problem in a somewhat different way from these authors, and more in accord with our previous considerations.

${ }^{44}$ P. Rona and D. Takahashi, Biochem. Zeitschr. 49, 370 (1913). 
The partial solubility of the molecular species $\mathrm{CaCO}_{3}$ is, independently of the $\left[\mathrm{H}^{+}\right],=\lambda$. Therefore, the total solubility is

$$
\Lambda=\lambda+\left[\mathrm{Ca}^{++}\right]
$$

where $\left[\mathrm{Ca}^{++}\right]$is that concentration of $\mathrm{Ca}^{++}$ions which is in equilibrium with the $\mathrm{CaCO}_{3}$ concentration $\lambda$. This $\left[\mathrm{Ca}^{++}\right]$depends upon $\left[\mathrm{H}^{+}\right]$in the following way:

In solution there exists a state of equilibrium between calcium monocarbonate and calcium bicarbonate.

$$
\mathrm{CaCO}_{3}+\mathrm{H}_{2} \mathrm{CO}_{3} \rightleftharpoons \mathrm{Ca}\left(\mathrm{HCO}_{3}\right)_{2}
$$

or

$$
\mathrm{CaCO}_{3}+\mathrm{H}_{2} \mathrm{CO}_{3} \rightleftharpoons \mathrm{Ca}^{++}+2 \mathrm{HCO}_{3}^{-}
$$

Therefore,

$$
\frac{\left[\mathrm{CaCO}_{3}\right]\left[\mathrm{H}_{2} \mathrm{CO}_{3}\right]}{\left[\mathrm{Ca}^{++}\right]\left[\mathrm{HCO}_{3}^{-}\right]^{2}}=\mathrm{k}_{1}
$$

But in the solution, $\left[\mathrm{CaCO}_{3}\right]=\lambda$

Furthermore,

$$
\mathrm{H}_{2} \mathrm{CO}_{3} \rightleftharpoons \mathrm{H}^{+}+\mathrm{HCO}_{3}^{-}
$$

hence

$$
\frac{\left[\mathrm{H}_{2} \mathrm{CO}_{3}\right]}{\left[\mathrm{HCO}_{3}^{-}\right]}=\mathrm{k}_{2} \times\left[\mathrm{H}^{+}\right]
$$

Substituting in (1) the value of $\left[\mathrm{H}_{2} \mathrm{CO}_{3}\right]$ found in (2),

$$
\frac{\lambda \times \mathrm{k}_{2} \times\left[\mathrm{H}^{+}[\right.}{\left[\mathrm{Ca}^{++}\right] \times\left[\mathrm{HCO}_{3}^{-}\right]}=\mathrm{k}_{1}
$$

or,

$$
\left[\mathrm{Ca}^{++}\right]=\frac{\lambda \mathrm{k}_{2}}{\mathrm{k}_{1}} \times \frac{\left[\mathrm{H}^{+}\right]}{\left[\mathrm{HCO}_{3}^{-}\right]}
$$

and

$$
\Lambda=\lambda+\frac{\lambda \mathrm{k}_{2}}{\mathrm{k}_{1}} \times \frac{\left[\mathrm{H}^{+}\right]}{\left[\mathrm{HCO}_{3}{ }^{-}\right]}
$$

It is evident then that the solubility of $\mathrm{CaCO}_{3}$ depends not only upon the $\left[\mathrm{H}^{+}\right]$but also upon the concentration of the bicarbonate 
ions present in the solution, regardless whether these later are derived from the solid phase or are added to the solution extraneously as $\mathrm{NaHCO}_{3}$. Analogously to the assumption of certain approximations in the first part of this book, we may assume the concentration of the bicarbonate ions as being equal to that of the total bicarbonate in solution. Furthermore it can be shown by means of a solubility determination in a strongly alkaline reaction that $\lambda$ is no longer analytically measurable and that practically $\lambda=0$. The above equation is, therefore, simplified to the form

$$
\Lambda=K \frac{\left[\mathrm{H}^{+}\right]}{\left[\mathrm{HCO}_{3}^{-}\right]}
$$

The following values were found for $\Lambda$ at $18^{\circ}$ :

\begin{tabular}{c|c|c|c}
\hline$\left[\mathrm{HCO}_{3}-\right] \times 0.9^{*}$ & {$\left[\mathrm{H}^{+}\right]$} & $\begin{array}{c}\Lambda \\
(\mathrm{mol} / \mathrm{liter})\end{array}$ & $\mathrm{K}$ \\
\hline 0.017 & $2.4 \times 10^{-7}$ & $4.93 \times 10^{-3}$ & 349 \\
0.0425 & $9.0 \times 10^{-8}$ & $7.3 \times 10^{-4}$ & 345 \\
0.0152 & $9.3 \times 10^{-8}$ & $2.03 \times 10^{-3}$ & 332 \\
\hline Average $\ldots \ldots \ldots \ldots \ldots \ldots \ldots \ldots \ldots \ldots \ldots \ldots \ldots \ldots \ldots \ldots \ldots \ldots \ldots \ldots$ & 312 \\
\hline
\end{tabular}

Therefore, at $18^{\circ}$

$$
\Lambda=342 \times \frac{\left[\mathrm{H}^{+}\right]}{\left[\mathrm{HCO}_{3}{ }^{-}\right]} \text {mols per liter }
$$

For the conditions existing in the blood plasma, where $\left[\mathrm{H}^{+}\right]$ $=3 \times 10^{-8}$ and $\left[\mathrm{HCO}_{3}^{-}\right]=0.02 \mathrm{~N}$, a solubility of $0.022 \mathrm{~g} . \mathrm{Ca}^{++}$ or $0.0294 \mathrm{~g}$. $\mathrm{CaO}$ per liter is calculated. In reality one finds more $\mathrm{CaO}$ in the plasma, at all events more than $0.1 \mathrm{~g}$., and at times even $0.17 \mathrm{~g}$. $\mathrm{CaO}$ per liter. In the whole blood there is about $0.1 \mathrm{~g}$. $\mathrm{CaO}$ per liter. It appears, therefore, that the plasma contains more $\mathrm{Ca}$ than the amount corresponding to the solubility of $\mathrm{CaCO}_{3}$, and it seems as if this were a case of a supersaturated solution. This assumption is all the more plausible, since in practice it is quite easy to prepare such supersaturated lime solutions, even in the absence of protein. Thus, when instead of solid $\mathrm{CaCO}_{3}$ a $\mathrm{CaCl}_{2}$ solution is

* This is a correction factor which must be introduced in order to take account of the fact that the active mass of the bicarbonate ions is somewhat smaller than the concentration of the calcium bicarbonate. 
used and is rendered slightly alkaline with $\mathrm{NaHCO}_{3}$, a clear stable supersaturated lime solution is obtained. Dialysis experiments have demonstrated that the greater portion of the $\mathrm{Ca}$ in scrum is present in a dialysable form. This excludes the possibility that the excess of calcium in the serum could be present in the form of a colloidal suspension or as a solution of a carbonate or phosphate. That portion of the calcium which in the compensation dialysis experiments was found to be non-diffusible is but a small fraction of the excess calcium present, and this portion only may be regarded as combined in an undissociated calcium-protein compound.

Brinkman and van Dam, ${ }^{45}$ using another method, confirmed the solubility data of Rona and Takahashi $(22 \mathrm{mg}$. Ca++ per liter, for $\left[\mathrm{H}^{+}\right]=3 \times 10^{-8}$ and $20^{\circ}$ ), and they also found $22 \mathrm{mg}$. $\mathrm{Ca}^{++}$ions in the ultrafiltrate of blood serum at $\left[\mathrm{H}^{+}\right]=3 \times 10^{-8}$. In the serum itself, in those few cases in which their method could be applied, they found the same amount of $\mathrm{Ca}^{++}$ions. If Rona's compensation dialysate and Brinkman's ultrafiltrate be regarded as being to a certain degree the same, then all of these results may be explained only on the following assumption. The ionized calcium is present in the serum in a concentration within the limit of its actual solubility and not in a supersaturated state, and that the excess calcium is present but to a small extent as a protein complex and largely in true solution in an undissociated form of unknown nature.

The following further comment is to be made upon these investigations. In the first place Rona's as well as Brinkman's solubility determinations apply to a temperature of $20^{\circ}$. Secondly they assume the $\left[\mathrm{H}^{+}\right]$in blood to be $3 \times 10^{-8}$, whereas for $38^{\circ}$ it should be taken as $4.5 \times 10^{-8}$. Thirdly, it must be pointed out that the theory of activity, which is to be discussed in the next chapter, could not yet have been taken into consideration, and it is probable that the factor of 0.9 (see footnote on page 84) is too low.

${ }^{45}$ R. Brinkman and E. van Dam. Kon. Akad. Wet. Amsterdam 22, 762 (1920). The method used by these authors for the determination of $\mathrm{Ca}^{++}$ ions consisted of establishing the amount of oxalate to be added to a solution containing calcium in orcler just to exceed the limit of solubility of the calcium oxalate produced. Since the solubility product $\left[\mathrm{Ca}^{++}\right] \times$[oxalate- $]$is constant $\left(0.055\right.$ millimols per liter at $\left.20^{\circ}\right)$ it is possible from the above amount of the added alkali oxalate to calculate the $\mathrm{Ca}^{++}$present. All the other methods of estimating calcium determine the total calcium. 
These three conditions all point in the direction of the probability that the solubility of calcium as determined in these experiments is too low. It is possible that the discrepancy between the solubility of calcium in plasma as based on theory and its actual calcium content is not as great as these investigations appear to indicate.

It may be seen from the above example how much more important the exact knowledge of natural constants is than hypotheses are for the clear conception of physiological facts. It is surprising that such physiologically important constants have not yet been determined. The theories of formation of calculi and of the deposition of lime in the blood vessels are truly phantastic, as long as it is not established whether or not the calcium of the blood is at all in a supersaturated state.

How complicated the previously discussed problem of uric acid is may be appreciated at this juncture, when it is recalled that in this case the slight solubility of the free acid is combined with the slight solubility of its Na-salt. The preceding chapter concerned itself with a slightly soluble acid whose salts are easily soluble (benzoic acid), while the present chapter deals with slightly soluble salts whose components $\left(\mathrm{CO}_{2}, \mathrm{Ca}(\mathrm{HO})_{2}\right)$ are more soluble than the salt. In the case of uric acid we have a condition not yet theoretically investigated, where both the free acid and its salts are but slightly soluble.

\section{The relation of the hydrion concentration to the solubility of a slightly soluble ampholyte}

If the partial solubility of the slightly soluble ampholyte bc $=$ $\lambda$, then the total solubility is

$$
\Lambda=\lambda+A^{+}+A^{-}
$$

where $\mathrm{A}^{+}$and $\mathrm{A}^{-}$denote the ampholyte in its cationic and anionie forms respectively.

The experimental investigations apply here insofar as the solubility minimum has been studied. This condition obtains when $\left[\mathrm{A}^{+}\right]+\left[\mathrm{A}^{-}\right]$is at a minimum, therefore, at the isoelectric point. Thus we return to the consideration fully discussed previously on page 70 .

Finally it must be recalled once more that all of this discussion 
of the solubility is made on the approximate assumption that the salts of the slightly soluble acids or bases are totally dissociated. As soon as the presence of appreciable amounts of undissociated salts is taken into account, then certain corrections must be introduced, which will be discussed in a later chapter dealing with strong electrolytes and the ionic activity theory. All of the above deductions apply only to such cases in which the concentration of the undissociated electrolytes in the solution in question remains quite small.

\section{The hydrolysis of salts ${ }^{46}$}

It has been assumed throughout above that all salts are completely electrolytically dissociated. But the salts have also another form of dissociation. Since a salt is produced by the general reaction

$$
\text { Acid }+ \text { base } \rightleftharpoons \text { salt }+\mathrm{H}_{2} \mathrm{O}
$$

we must assume, in accord with the theory of equilibrium, that this reaction also takes place in the opposite direction, i.e., that the salt partially "dissociates hydrolytically" or "hydrolyses" to form acid and base. It is obviously imperative to establish the degree or extent of this hydrolysis.

First case. The salt of a strong acid and a strong base. If the acid be designated as $\mathrm{AH}$, the base as $\mathrm{BOH}$ and the salt as $\mathrm{C}$, then according to our assumption we have present in solution only the ions $\mathrm{A}^{-}, \mathrm{B}^{+}, \mathrm{H}^{+}$and $\mathrm{OH}^{-}$. Since $\mathrm{A}^{-}$and $\mathrm{B}^{+}$in a strong acid and base have no affinity for the $\mathrm{H}^{+}$and $\mathrm{OH}^{-}$ions, it follows that they have no effect upon the $\left[\mathrm{H}^{+}\right]$and $\left[\mathrm{OH}^{-}\right]$, that is to say, in the aqueous solution the hydrion concentration is not affected by such a salt. These salts are not at all hydrolyzed, and the solution of a salt of this type is neutral in reaction. These salts are therefore called neutral salts.

Second case. The salt of a strong acid and a weak base whose dissociation constant is $\mathrm{k}_{\mathrm{b}}$. In this case the salt is hydrolyzed to a small extent with the formation of a little free acid and free base. But since the base dissociates less than the acid, the solution has a

${ }^{46}$ Taken chiefly from N. Bjerrum. Die acidimetrische and alkalimetrische Titration. Stuttgart. 1914. 
slightly acid reaction. Let us now study the degree of hydrolysis $\gamma$ of the salt which is defined as

$$
\gamma=\frac{\left[\mathrm{H}^{+}\right]}{[\mathrm{C}]}
$$

where $[\mathrm{C}]$ denotes the total concentration of the salt.

For the general solution of the problem let us consider the coexistence of the following laws:

$$
\left[\mathrm{H}^{+}\right] \times\left[\mathrm{OH}^{-}\right]=\mathrm{k}_{\mathrm{w}}
$$

which is the dissociation equation of water.

Since the acid produced in the hydrolysis was assumed to be a strong acid, its concentration is therefore $=\left[\mathrm{H}^{+}\right]$. But since in the hydrolysis as much base is produced as acid, it follows that

$$
\left[\mathrm{H}^{+}\right]=\left[\mathrm{OH}^{-}\right]+[\mathrm{BOH}]
$$

Furthermore the equation for the dissociation of the base is

$$
\frac{\left[\mathrm{B}^{+}\right]\left[\mathrm{OH}^{-}\right]}{[\mathrm{BOH}]}=\mathrm{k}_{\mathrm{b}}
$$

and finally,

$$
\left[\mathrm{B}^{+}\right]+[\mathrm{BOH}]=[\mathrm{C}]
$$

From these four equations involving the four unknown values $\left[\mathrm{H}^{+}\right],\left[\mathrm{OH}^{-}\right],\left[\mathrm{B}^{+}\right]$and $[\mathrm{BOH}]$ each of the latter may be calculated, and knowing the value of $\left[\mathrm{H}^{+}\right]$that of $\gamma$ may be obtained. But these calculations are of a rather involved character. It suffices ordinarily to carry out the calculations, with some simplifying assumptions, for certain limits, as:

a. Assuming that the hydrolysis is very slight $\left(\gamma<0.01,\left[\mathrm{H}^{+}\right]\right.$ $<10^{-3}$ ).

Then in equation (4) the value of $[\mathrm{BOH}]$ as a term of a sum is negligible in respect to that of $\left[\mathrm{B}^{+}\right]$and in this case

$$
\left[\mathrm{B}^{+}\right]=[\mathrm{C}]
$$

and solving for $\left[\mathrm{H}^{+}\right]$by substituting in (2) the value for $\left[\mathrm{OH}^{-}\right]$from 
(1) and the value for [BOH] from (3), and by substituting [C] for $\left[\mathrm{B}^{+}\right]$as in (4a), we obtain:

$$
\begin{gathered}
{\left[\mathrm{H}^{+}\right]^{2}=[\mathrm{C}] \frac{\mathrm{k}_{\mathrm{w}}}{\mathrm{k}_{\mathrm{b}}}+\mathrm{k}_{\mathrm{w}}} \\
\gamma=\sqrt{\frac{1}{[\mathrm{C}]} \times \frac{\mathrm{k}_{\mathrm{w}}}{\mathrm{k}_{\mathrm{b}}}\left(1+\frac{\mathrm{k}_{\mathrm{b}}}{[\mathrm{C}]}\right)}
\end{gathered}
$$

b. Assuming a moderate hydrolysis $\left(\gamma>0.01\right.$ and $\left.\left[\mathrm{H}^{+}\right]>10^{-5}\right)$ we may disregard the value of $\left[\mathrm{OH}^{-}\right]$in equation (2) in respect to the other terms of the sum $[\mathrm{BOH}]$. Thus the equation becomes

$$
\left[\mathrm{H}^{+}\right]=[\mathrm{BOH}]
$$

and furthermore the value for $\left[\mathrm{B}^{+}\right]$in $(4)$ still remains closely enough under the conditions:

$$
\left[\mathrm{B}^{+}\right]=[\mathrm{C}]
$$

on substituting these two values in (3) we obtain

$$
\left[\mathrm{H}^{+}\right]^{2}=\frac{[\mathrm{C}] \times \mathrm{k}_{\mathrm{W}}}{\mathrm{k}_{\mathrm{b}}}
$$

and

$$
\gamma=\sqrt{\frac{1}{[C]} \times \frac{k_{w}}{k_{b}}}
$$

c. In the case of strong hydrolysis we may likewise assume for all purposes instead of equation (2) that

$$
\left[\mathrm{H}^{+}\right]=[\mathrm{BOH}]
$$

from which we derive:

$$
\left[\mathrm{H}^{+}\right]^{2}=[\mathrm{C}] \cdot \frac{\mathrm{k}_{\mathrm{w}}}{\mathrm{k}_{\mathrm{b}}}-\left[\mathrm{H}^{+}\right] \cdot \frac{\mathrm{k}_{\mathrm{w}}}{\mathrm{k}_{\mathrm{b}}}
$$

and

$$
\gamma=\frac{\sqrt{\frac{1}{[C]} \cdot \frac{k_{w}}{k_{b}}}}{\sqrt{1+\frac{k_{w}}{4[C] \cdot k_{b}}}+\sqrt{\frac{k_{w}}{4[C] \cdot k_{b}}}}
$$


If the salt is the product of a strong base and a weak acid whose dissociation constant is $\mathrm{k}_{\mathrm{a}}$, then the same relationships as given above are applicable except that $\left[\mathrm{OH}^{-}\right]$is substituted for $\left[\mathrm{H}^{+}\right]$and the degree of hydrolysis is defined as

$$
\gamma=\frac{\left[\mathrm{OH}^{-}\right]}{[\mathrm{C}]}
$$

TABLE 16

Table of values for the degree of hydrolysis at room temperature

\begin{tabular}{|c|c|c|c|c|}
\hline \multirow{2}{*}{$k$} & \multicolumn{4}{|c|}{$k_{w}=10^{-14}$} \\
\hline & $1 \mathrm{~N}$ & $0.1 \mathrm{~N}$ & $0.01 \mathrm{~N}$ & $0.001 N$ \\
\hline$\infty$ & $10^{-7}$ & $10^{-6}$ & $10^{-5}$ & $10^{-4}$ \\
\hline $10^{-2}$ & $10^{-6}$ & $3.3 \times 10^{-6}$ & $1.4 \times 10^{-5}$ & $1.05 \times 10^{-4}$ \\
\hline $10^{-4}$ & $10^{-5}$ & $3.2 \times 10^{-5}$ & $10^{-4}$ & $3.3 \times 10^{-4}$ \\
\hline $10^{-6}$ & $10^{-4}$ & $3.2 \times 10^{-4}$ & $10^{-3}$ & $3.2 \times 10^{-3}$ \\
\hline $10^{-8}$ & $10^{-3}$ & $3.2 \times 10^{-3}$ & $10^{-2}$ & 0.032 \\
\hline $10^{-10}$ & $10^{-2}$ & 0.032 & 0.095 & 0.27 \\
\hline $10^{-12}$ & 0.095 & 0.27 & 0.62 & 0.92 \\
\hline
\end{tabular}

TABLE 17

Table of $p H$ values in solutions of hydrolytic salts

$\mathrm{pH}$ values in the solution of a salt of a weak base and a strong acid, or pOHvalues in the solution of a salt of a strong base and a weak acid. $K$ is the dis sociation constant of the weaker constituent.

\begin{tabular}{c|c|c|c|c}
\hline $\mathrm{k}$ & $1 N$ & $0.1 \mathrm{~N}$ & $0.01 N$ & $0.001 \mathrm{~N}$ \\
\cline { 2 - 4 } & 7.0 & 7.0 & 7.0 & 7.0 \\
\cline { 3 - 4 } $10^{-2}$ & 6.0 & 6.5 & 6.9 & 7.0 \\
$10^{-4}$ & 5.0 & 5.5 & 6.0 & 7.0 \\
$10^{-6}$ & 4.0 & 4.5 & 5.0 & 6.0 \\
$10^{-8}$ & 3.0 & 3.5 & 4.0 & 4.5 \\
$10^{-10}$ & 2.0 & 2.5 & 3.0 & 3.6 \\
$10^{-12}$ & 1.02 & 1.6 & 2.2 & 3.0 \\
\hline
\end{tabular}

In table 16 the values given by Bjerrum of the degree of hydrolysis were calculated for various values of the dissociation constants, the latter given in round figures.

The values above the upper stair-line are obtained from equation (6), the values between the stair-lines from equation (8) and 
those below from equation (10). From the practical standpoint it appears even more important to tabulate the $\mathrm{pH}$ values of such solutions. These have been calculated by the author and are shown in table 17 .

Thus in solutions of $\mathrm{NH}_{4} \mathrm{Cl}\left(\mathrm{k}_{\mathrm{b}}\right.$ approximately $\left.=10^{-5}\right)$ and of sodium acetate $\left(\mathrm{k}_{\mathrm{a}}\right.$ approximately $\left.=10^{-5}\right)$ the following the $\mathrm{pH}$ values obtain:

\begin{tabular}{|c|c|c|c|c|}
\hline C & $1 . M$ & $0.1 \mathrm{H}$ & $0.01 M$ & $0.001 M$ \\
\hline $\mathrm{NH}_{4} \mathrm{Cl} \ldots \ldots \ldots \ldots \ldots \ldots$ & 4.5 & 5 & 5.5 & 6.5 \\
\hline Na-acetate............ & 9.5 & 9 & 8.5 & 7.5 \\
\hline
\end{tabular}

25. Notes on the methods for determining dissociation constants and the isoelectric point

1. The oldest method for the determination (f the dissociation constant of a simple electrolyte consists of measuring the conductivity of its solutions in various dilutions. Wilheln Ostwald found empirically that

$$
\frac{\alpha^{2}}{(1-\alpha) \mathrm{v}}=\mathrm{k}
$$

where $\alpha$ is the degree of dissociation at the concentration $\frac{1}{\mathrm{v}}, \mathrm{v}$ being that volume of solution that contains one mol of the solute. The value of $\alpha$ is determined by the expression

$$
\frac{\Lambda_{c}}{\Lambda_{\infty}}=\alpha
$$

where $\Lambda_{c}$ is the molar conductance at the concentration $c$ and $\Lambda_{\infty}$ denotes its limiting value at infinite dilution. Ostwald's law, as given in equation (1) is but an expression of the mass law applied to dissociation. According to definition, for instance, it is true for an acid that

$$
\alpha=\frac{\mathrm{S}^{-}}{\mathrm{S}}
$$


where $\mathrm{S}^{-}$is the concentration of the acid anions and $\mathrm{S}$ is that of the total acid.

According to the mass law

$$
\frac{\left[\mathrm{S}^{-}\right] \times\left[\mathrm{H}^{+}\right]}{[\mathrm{S}]-\left[\mathrm{S}^{-}\right]}=\mathrm{k}
$$

If in the above expression we substitute for the concentration $\left[\mathrm{S}^{-}\right]$its equivalent $\frac{\mathrm{S}^{-}}{\mathrm{v}}$, where $\mathrm{v}$ is the volume and $\mathrm{S}^{-}$is the absolute molar amount of the anions, and by recalling that in a pure acid solution $\left[\mathrm{S}^{-}\right]=\left[\mathrm{H}^{+}\right]$, we obtain

$$
\frac{\left(\frac{S^{-}}{v}\right)^{2}}{\frac{S-S^{-}}{v}}=\frac{\left(S^{-}\right)^{2}}{\left(S-S^{-}\right) v}=\frac{\alpha^{2}}{(1-\alpha) v}=k
$$

2. The second method depends upon the determination of the degree of hydrolysis of the sodium salt of the weak base or the chloride of the weak acid studied. The relation of this factor to $\mathrm{k}$ follows obviously from the preceding chapter. The degree of hydrolysis may be estimated by two methods:

$a$. By determining the $\left[\mathrm{H}^{+}\right]$from the catalytic action on the hydrolysis of sucrose, methyl acetate, etc.

$b$. By the electrometric determination of the $\left[\mathrm{H}^{+}\right]$. This method has been little employed.

3. The third method depends upon the determination of $\left[\mathrm{H}^{+}\right]$ in a definite mixture of the acid with its Na-salt, for which purposes various methods may be employed. The theoretical basis of this procedure is given by formula (1) on page 44 .

Each of these methods has its own field of applicability. But there are conditions under which either the experimental data become uncertain, or, where the equations which are but approximations applying within certain limits are no longer applicable. It is beyond the scope of this book to deal with this question in greater detail. Neither are all the possible methods exhausted with those given above. Thus, for instance, a very good method for an acid 
of moderate strength is to measure the $\left[\mathrm{H}^{+}\right]$in a pure solution of the acid and to calculate $\mathrm{k}$ by applying equation (1a) on page 40 . The second method given above has been developed by Lundén who determined the degree of hydrolysis of the salt of the acid studied and of a weak base of a known dissociation constant. 


\section{CHAPTER II}

The Theory of the Quantitative Determination of Acidity AND Alkalinity

\section{SUMMARY OF CONTENTS}

For the characterization of the acid nature of a solution it is necessary to know: (1) The titratable acidity which may be further subdivided into neutralizing capacity and equivalent capacity, (2), the hydrogen ion concentration or $\mathrm{pH}$ value, and (3) the buffer value.

The theory of indicators and of the titration curve is developed for the elucidation of the concept of titratable acidity; the principles of the most important methods for the estimation of the $\mathrm{pH}$ are then briefly outlined; and finally the conception of buffer value is developed.

\section{Definitions and statement of problem}

The acidity of a solution is defined by a number of values, and the sharp demarcation and differentiation of these values has become possible with the advent of physico-chemical methods. In the older analytical chemistry these values were either not differentiated at all, or else to an insufficient extent. It is true that in previous decades excellent methods were found for the estimation of acidity and alkalinity in the form of the titration, but the reason for this is due to the fact that for strong acids and bases the various values which define acidity practically coincide. Furthermore, for certain of the weaker acids and bases some indicators had been empirically found, such as phenolphthalein and methyl orange, which enabled our predecessors to determine the equivalent content of these acids and bases. Thus experiment had demonstrated that the equivalent content of an acetic acid solution could be correctly determined by a titration against a standard $\mathrm{NaOH}$ solution with phenolphthalein, that an incorrect value was obtained when methyl orange was used as an indicator in the same titration, and that the reverse was true for the titration of ammonia against sulfuric acid. At any rate, the acidity of a solution had been defined by its content of acid equivalents. 
Therefore, according to this definition a normal hydrochloric acid and a normal acetic acid solution were of exactly the same acidity. On the other hand, it could not be entirely ignored that these two equivalent acid solutions were in some respects quite different, and a stronger acidic property had to be conceded to the hydrochloric acid than to the acetic acid. There were obviously two entirely different properties which were designated as acidity, and only one of these properties could be quantitatively estimated by means of the titration. For the other property there were only qualitative approximations. Thus it was known, for instance, that normal hydrochloric acid attacked and dissolved zinc better than normal acetic acid, that the former changed congo-red paper to a bluer tint, that it had a more corrosively acid taste, that it inverted cane sugar more rapidly, etc.

By applying the principles of the ionic theory we may now define acidity quantitatively in three ways, which do away with the above described uncertainties. Furthermore, we shall learn of a fourth value or factor which is necessary for the complete characterization of the acid properties of a solution.

One of our new definitions, "titratable acidity," corresponds somewhat to the older definition. It is the number of equivalents of free acid in the solution. But as soon as we wish to define the methods by which these equivalents are determined, we find that the older conception is inadequate. For, if we were to ask a chemist of a few decades ago to what endpoint was an acid solution to be titrated he would say: until the neutral reaction was reached; and as a means of recognizing this point he would probably suggest the change of color of phenolphthalein. We now know that such a reply contains two errors. In the first place, a solution consisting of equivalent parts of acetic acid and $\mathrm{NaOH}$, or of sodium acetate, is not neutral, but, because of the ensuing hydrolysis, it is somewhat alkaline (see table, page 80 ). Secondly, the turning point of phenolphthalein is not at the neutral, but at an alkaline reaction. Therefore, our analyst will in the end be practically quite correct, in spite of his erroneous explanation. But he could not have explained why he should not have just as well used methyl orange in his titration. At best he would say that "methyl orange is not sensitive enough to acetic acid" without being able to explain why it was more sensitive to hydrochloric acid.

In reality the endpoint of the titration of acetic acid is not at the 
neutral reaction, but at the hydrion concentration corresponding to that of a solution of sodium acetate, which in turn corresponds to the turning point of phenolphthalein, but not to that of methyl orange. If we should wish to attribute a significance to the actual point of neutrality then we would have to differentiate two endpoints in our titration: first, the attainment of the neutral point and secondly the reaching of the point of equivalence. The number of equivalents of the base which were used up in reaching the neutral point may be designated as the neutralizing capacity and the number of base equivalents used to reach the point of equivalence as the equivalent capacity, or, the true titration capacity. It is only when a strong acid is titrated against a strong base that these two conceptions coincide. We must now learn to know the methods by means of which these two values are determined.

\section{The determination of neutralizing capacity}

For the estimation of this point an indicator is needed which shows a definite color change at the neutral reaction. Such indicators are, litmus (solution), neutral red, or phenol red. Exactly neutral water is colored with litmus, and the investigated acid solution is titrated until it shows the same blue-violet shade of color of its litmus as the standard solution.

Since it is very difficult to obtain really pure water, it is more practicable to employ instead of the water a mixture consisting of 6.9 volumes of $\mathrm{M} / 15 \mathrm{Na}_{2} \mathrm{HPO}_{4}$ and 3.1 volumes of $\mathrm{M} / 15 \mathrm{KH}_{2} \mathrm{PO}_{4}$ solutions. The reaction of such a mixture is exactly neutral at $18^{\circ}$, according to Sørensen.

The determination of the neutralizing capacity is of little practical importance.

\section{The determination of the equivalent capacity}

For the discussion of this topic a.preliminary and more detailed explanation of the theory of indicators is required, which follows.

\section{The theory of indicators}

The indicators are acids or bases whose shade of color is changed by the hydrion concentration of the solution. Wilhelm Ostwald'

1 W. Ostwald, Lehrb. d. allgem. Chem., 1891, p. 799 and Z. physikal. Chem 9, 579 (1892). 
has long ago established the theory, which in its essentials is still valid, and according to which this change is explained by the assumption that the ions of a dye-stuff possess a color different from that of its undissociated molecule. An apparent objection to this theory arose from the work of Hantzsch ${ }^{2}$ who showed indicators to be pseudoacids and bases, and according to whom the change of color is not due to ionization but to tautomeric rearrangement. But since these two processes are inseparably related to each other, no real contradiction can be found in these two theories. The theory of Hantzsch serves only to elucidate further the mechanism by which the color changes of indicators occur. We may even go further in the same direction. In accord with the conception of the atomic model the configuration of the atoms in the molecule is determined by electrostatic forces. It is, therefore, not surprising that the appearance of a free charge, as it occurs in the process of ion formation, may recast the configuration of the atoms in the molecule. Thus we can still retain the older conception and state that an indicator is a substance which is characterized by a difference in color between its unionized and its ionized states.

For practical purposes two groups of indicators are recognized: the one-color indicators which change from a colorless to a colored state, as phenolphthalein, and the two-color indicators, such as litmus, which change from one color to another. It is more convenient in the development of the theory to begin with the one-color indicators. Let us choose, as an example, $p$-nitrophenol. This is an acid whose dissociation constant, $\mathrm{k}_{\mathrm{a}}=6.5 \times 10^{-8}$, or, $\mathrm{pk}=7.19$. The free acid is colorless, while its ions are yellow. Therefore at any given $\left[\mathrm{H}^{+}\right]$a part of this dye is present as the colorless free acid and another part in the form of yellow colored ions. The proportion of the ions to the total amount of indicator in solution is its degree of dissociation, or $\alpha$. This latter value can be quite easily determined experimentally. Thus we may place the same amount of indicator into a second vessel and render it strongly alkaline with sodium hydroxide in which case the indicator will be completely dissociated and show its maximum color intensity. If now we compare colorimetrically the color intensity of the first solution with that of the second, and if this numerical relation of the concentration of the indicator be designated as the "degree of color," or, as F, then $\mathrm{F}=\alpha$.

${ }^{2}$ H. Hantzsch, Ber. deut. chem. Ges. 46, 1537 (1913); ibid. 48, 158 (1915). 
It follows then that $\alpha$ as well as $\mathrm{F}$ are functions of the $\mathrm{pH}$, of the type of the dissociation curve.

Figure 11 shows one of such curves for $p$-nitrophenol, which has already been discussed on page 54 . As will be readily seen this indicator is practically colorless in the $\mathrm{pH}$ range of 0 to 5.0. The $\mathrm{pH}$ at which it begins to show color depends upon its concentration. It may be stated in a general way that with concentrations of indicators usually employed in titrations the color becomes noticeable when the indicator is 5 per cent dissociated. In this particular case, this occurs at about $\mathrm{pH} 5.2$, at most at $\mathrm{pH}$ 6.0. This so-called transformation point of the indicator does not in any way correspond with the

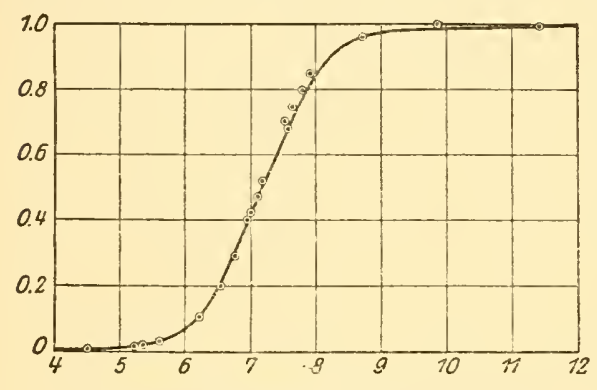

FIg. 11. The abscissa represent $\mathrm{pH}$ values and the ordinates represent the color intensity of $p$-nitrophenol. The curve as drawn is the calculated theoretical dissociation-( $\alpha)$-curve of the indicator, the circles are the experimentally observed values.

consider it as remaining constant. This assumption simplifies the calculation and introduces no marked error. In figure 12 the amount of the alkali added in cubic centimeters is shown on the abscissa and the resulting $\mathrm{pH}$ on the ordinate. The latter is calculated on the assumption that the acid, the alkali and the salt formed are always completely dissociated and that the amount of indicator added is so small that it does not influence the state of equilibrium between the acid and the base.

In the beginning of the experiment we have $0.1 \mathrm{~N} \mathrm{HCl}$, therefore the $\mathrm{pH}=1.0$. After the addition of $1 \mathrm{cc}$. of the base we have a $0.09 N \mathrm{HCl}$ solution, or $\mathrm{pH}=1.05$. In this manner we may continue our calculation until we approach the vicinity of the neutral 
point. Having added 9.5 cc. $N$ NaOH we still have a $0.005 N \mathrm{HCl}$ solution, $\mathrm{pH} 2.3$. With $10 \mathrm{cc}$. of the base added we have neutrality or $\mathrm{pH} 7$. On the further addition of the alkali free base is present in solution and the indifferent $\mathrm{NaCl}$. From this point we may calculate the resulting $\mathrm{pOH}$ and therefore the $\mathrm{pH}$. The lowest curve of figure 12 is obtained in this manner.

The curve is characterized by its initial flat or horizontal course and then by the sudden and abrupt rise at the "end-point of the titration." In this region the addition of a few drops of the alkali causes a steep increase of the $\mathrm{pH}$ from about 3.0 to 11.0. If we should ask what relation the transformation point of the nitrophenol $(\mathrm{pH} 5.0$

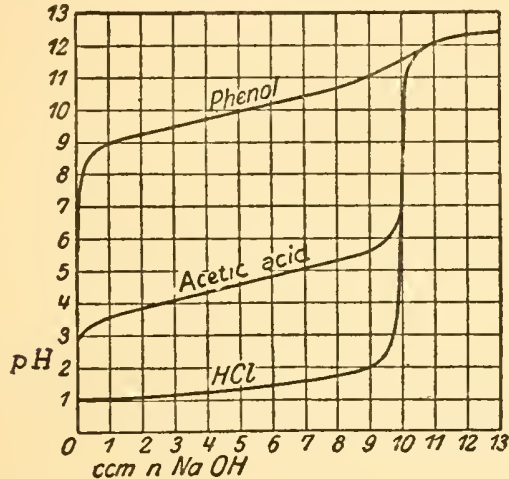

FIg. 12. Titration curves of 10 cc. portions of $N \mathrm{HCl}$, acetic acid and phenol with $N \mathrm{NaOH}$.

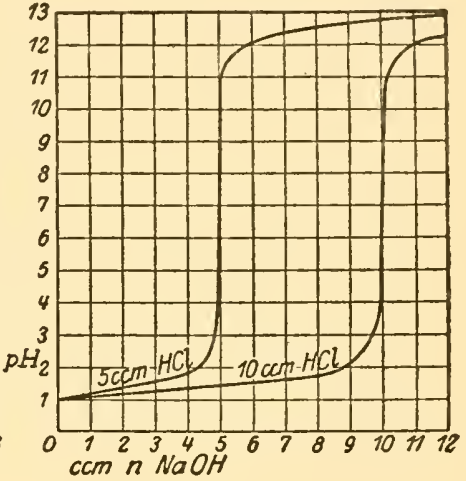

Fig, 13. Titration curves of 5 cc. and $10 \mathrm{cc}$. portions of $N \mathrm{HCl}$.

to 6.0 ) bears to this curve, we shall see at once that this point falls within the rapidly ascending portion of the curve. Thus $p$-nitrophenol gives us the correct end point of the titration. But it is also to be observed that the use of this particular indicator did not in any way mark the point $\mathrm{pH} 5.0$ to 6.0 . The same result could be obtained with an indicator whose transformation point is at $\mathrm{pH} 7.0$ (litmus) or $\mathrm{pH} 8.0$ (phenolphthalein). The amount of the alkali which in this titration changes the $\mathrm{pH}$ of the solution from 5.0 to 9.0 is less than one drop. From this we arrive at the rule: To titrate a strong acid with a strong base one may choose an indicator whose transformation point lies between $\mathrm{pH} 5.0$ and $\mathrm{pH} 9.0$. 
Second case: Titration of a weak acid with a strong base. Ten cubic centimeters of $N$ acetic acid are diluted with water to $100 \mathrm{cc}$. and titrated with $N \mathrm{NaOH}$. Again we construct a curve which represents the change in $\mathrm{pH}$ with increasing quantities of added base.

To calculate the $\mathrm{pH}$ values in this case the following principles are applied:

1. The $\mathrm{pH}$ of the pure acetic acid solution is calculated from equation (la) page 40.

$$
\left[\mathrm{H}^{+}\right]=\sqrt{\mathrm{k} \times[\mathrm{A}]}
$$

2. On the addition of $\mathrm{NaOH}$ we have in solution a mixture of free acetic acid + sodium acetate. The $\mathrm{pH}$ of these mixtures is derived from equation (1), page 44.

$$
\left[\mathrm{H}^{+}\right]=\mathrm{k} \frac{\text { [free acetic acid] }}{\text { [sodium acetate] }}
$$

3. As soon as an equivalent quantity of the base had been added we have a $0.1 N$ solution of pure sodium acetate. At this state the $\mathrm{pH}$ value is calculated from the hydrolysis formulae (see table, page 90).

4. When an excess of base had been added we deal with a dilute $\mathrm{NaOH}$ solution; assuming total dissociation the $\mathrm{pOH}$ and hence $\mathrm{pH}$ may be obtained.

In this manner the middle curve in figure 12 is obtained.

The end point in this titration is also characterized by a sudden rise in the $\mathrm{pH}$ curve. But here also the transformation point of the nitrophenol ( $\mathrm{pH} 5.0$ to 6.0 ) bears no relation to this sudden rise of the curve. This rise covers the range of $\mathrm{pH} 7.0$ to 11.0. Neither nitrophenol ( $\mathrm{pH}$ range 5.0 to 6.0 ) nor litmus ( $\mathrm{pH} 7.0$ ) but phenolphthalein alone $(\mathrm{pH} 8.0$ to 8.5$)$ can be used as an indicator for reaching the true end-point of this titration. The dissociation constant of phenolphthalein is $\mathrm{pk}=9.7$ and hence its transformation point is at about $\mathrm{pH} 8.0$ to 8.5 .

Among the weak acids acetic acid $\left(k=2 \times 10^{-5}\right)$ is a fairly strong one. If instead of acetic acid a still weaker acid, such as boric acid or phenol $(\mathrm{pk}=10)$, then an entirely different curve is obtained (upper curve in figure 12).

In this case the $\mathrm{pH}$ values are calculated just as in the case of acetic acid from the equations stated above. 
No definite sudden rise is observed in this case. The "equivalence point" lies at about pH 11.5. If a very weak acid of the general type of boric acid is to be titrated it can only be done in the following way: A suitable indicator, such as $m$-nitro-benzene-azosalicylic acid (Alizarine Yellow GG), is added to a pure solution of primary sodium borate, and an equal amount of the same indicator is added to the boric acid solution; the latter is then titrated until the same color as that in the sodium borate solution is reached. Since the rise on the $\mathrm{pH}$ curve through the point of equivalence is very small, the color change of the indicator is very gradual, and the titration could barely be carried out without the aid of a standard for comparison.

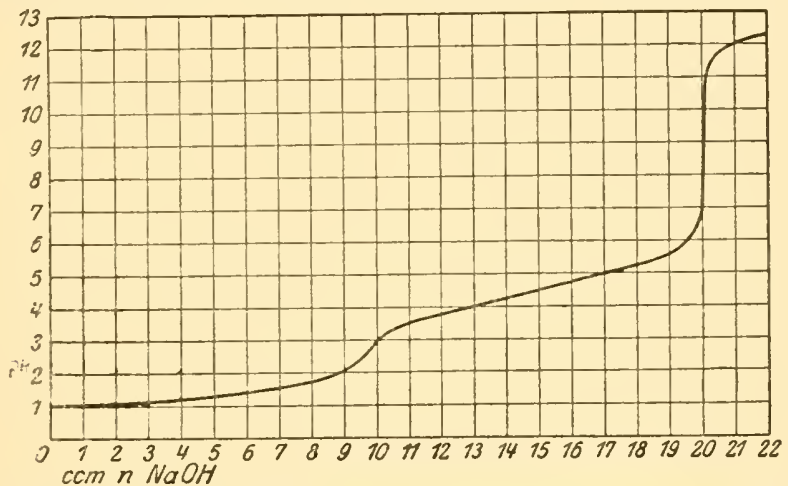

Fig. 14. Titration curve of a mixture consisting of $10 \mathrm{cc} . N \mathrm{HCl}+10 \mathrm{cc}$. $N$ acetic acid.

The limits of error of such titrations vary with the conditions. A theory of titration error has been advanced by Bjerrum. ${ }^{3}$ 'This theory may be roughly summarized by stating that the steeper the rise of the $\mathrm{pH}$ curve through the "point of equivalence" the smaller the error.

In connection with the above it is interesting to observe the behavior on titration of a mixture consisting of a strong and of a weak acid. Let us titrate a mixture containing $10 \mathrm{cc}$. of $N \mathrm{HCl}$ and 10 cc. of $N$ acetic acid in a volume of $100 \mathrm{cc}$. with $\mathrm{N} \mathrm{NaOH}$. The pH curve of this titration will follow the course shown in figure 14.

The calculation of the $\mathrm{pH}$ values is carried out as follows:

1. As long as free $\mathrm{HCl}$ is present it suppresses almost completely

${ }^{3}$ See footnote 7. 
the dissociation of the acetic acid, and the $\mathrm{pH}$ values are calculated from the free $\mathrm{HCl}$ alone.

2. As soon as the $\mathrm{HCl}$ is neutralized the calculation is the same as in the preceding case for $\mathrm{CH}_{3} \mathrm{COOH}+\mathrm{NaOH}$.

The curve shows a marked upward bend which corresponds to the disappearance of free $\mathrm{HCl}$ and which occurs at about $\mathrm{pH}$ 3.0. An indicator which marks off this $\mathrm{pH}$ value by a sharp color change is suitable for the determination of $\mathrm{HCl}$ in the mixture. $\alpha$-dinitrophenol, for example, is such an indicator, showing its first color change at about $\mathrm{pH}$ 3.0. If the titration is then continued with phenolphthalein, the acetic acid content may also be determined in addition.

There is no significant difference between a one-color and a twocolor indicator. In the case of the latter any observed mixed color consists of the optical mixture of the two individual different colored components. Thus, for intance, when a strongly acid red and a strongly alkaline blue litmus solution are placed one in front of the other so that one looks through both at the source of light, an optical impression is obtained of the same violet tint as would be produced by a litmus solution of an appropriate hydrion concentration. This topic will be more fully discussed in the part on methodology.

\section{The determination of the hydrion concentration, or the esti- mation of the actual acidity}

The determination of the hydrion concentration cannot be achieved by means of any titration method. The standard method used for this purpose is the measurement of the electromotive force developed in a hydrogen concentration chain. Schematically drawn this chain consists of two electrodes or half-elements, each being a piece of platinum saturated with hydrogen, and each of these electrodes is immersed into one of the two parts of a liquid system. ${ }^{4}$ The two parts of the liquid system are a solution of known $\left[\mathrm{H}^{+}\right]$and the unknown solution. The way in which the liquid junction between the solutions is arranged will be discussed in the part on methods. Let us at first assume that the known solution is 1 normal in respect to $\mathrm{H}$-ions, $\left[\mathrm{H}^{+}\right]=1$, or $\mathrm{pH}=0$. The electromotive force (E. M.F.)

${ }^{4}$ An excellent schematic illustration of the gas chain will be found in W. M. Clark, The Determination of Hydrogen Ions, 2nd edition, Baltimore, 1922, Fig. 13, p. 145. 
of such a concentration chain is according to Nernst's theory (the details of which will be discussed later)

$$
\text { E.M.F. }=R T \ln \frac{\mathrm{c}_{1}}{\mathrm{c}_{2}}
$$

in which formula $c_{1}$ and $c_{2}$ are the concentrations of the current-producing ions in the two solutions. All other ionic species but those that can be yielded by the electrodes (i.e., in this case all ions other than $\mathrm{H}$-ions) are of no significance. $\mathrm{R}$ is the gas constant, $\mathrm{T}$ the absolute temperature, and ln the natural logarithm. If the E.M.F. is to be given in volts and the logarithin converted from the natural into the common system then the value of $\mathrm{R}$ becomes $0.000,198,3$. $c_{1}$ being $=1$ and $c_{2}$ the desired $\left[\mathrm{H}^{+}\right]$in this case, we have for any temperature $\mathrm{T}$ in our concentration chain the relationship:

$$
\text { E. M. F. }=-0.000,198,3(\mathrm{t}+273) \log \left[\mathrm{H}^{+}\right]
$$

or for $18^{\circ} \mathrm{C}$.

$$
\text { E. M. F. }=-0.0577 \times \log \left[\mathrm{H}^{+}\right]
$$

hence

$$
\log \left[\mathrm{H}^{+}\right]=-\frac{\text { E.M.F. }}{0.0577}
$$

and

$$
\mathrm{pH}=\frac{\text { E.M.F }}{0.0577}
$$

Thus the measurement of the E.M.F. gives us the pH after mere division by a constant (for a given temperature) factor. The details of this method will be given in the part on experimental technic.

Another method for the determination of the hydrion concentration is the so-called indicator method. The use of the indicators in this case is quite different from that in the case of titration. The indicator is simply added to the solution in question and its $\mathrm{pH}$ is determined by the shade of color produced. This can be done in two ways. One method which was carefully worked out by Sorensen ${ }^{5}$ consists of matching the shade of the indicator color in the unknown against a known buffer solution showing the same shade of indicator color. The buffer solution is prepared by mixing certain known

5. P. L. Sørensen, Biochem. Zeitschr. 21, 131 (1909). 
stock solutions which can be easily reproduced, and the $\mathrm{pH}$ of such a mixture is once for all established by standardization in a concentration (gas) chain. The other method which was developed by the author ${ }^{6}$ depends upon the colorimetric estimation of the degree of color of one-color indicators. This is achieved by determining the relative amounts of indicator required to impart to the unknown solution and to an alkali solution the same shade of color. Since the shade of color depends upon the degree of color transformation and dissociation constant of the indicator (cf., page 97), the $\mathrm{pH}$ of the solution is obtained directly. No buffer solutions are required for this method. In its principle the method is also applicable to twocolor indicators, and in this form it was developed by Bjerrum ${ }^{7}$ and shortly before the author's work by Gillespie. ${ }^{8}$ And yet the procedure with one-color indicators is simpler, and the necessary one-color indicator's covering the entire $\mathrm{pH}$ range were first given by the author. Both indicator methods depend upon the gas chain method; the buffer method insofar as the buffer solutions must be standardized by the gas chain, and the method without buffers insofar as the determination of the dissociation constants of the indicators is best carried out by means of the gas chain method, although this can also be accomplished in other ways, such as by conductivity measurements. The details of the indicator methods will be fully described in the experimental part of this work.

A third, and historically the oldest, method is based upon the principle that the catalytic effect of a solution upon the velocity of certain chemical reactions depends upon its $\left[\mathrm{H}^{+}\right]$, as in the case of the hydrolysis of cane sugar, esters etc., or upon its $\left[\mathrm{OH}^{-}\right]$as in the case of the saponification of esters. This method will also be dwelt upon in greater detail in the part on methods.

\section{The resistance of solutions to change in reaction and buffer value $^{9}$}

The values for equivalent acid content, neutralizing capacity and the hydrion concentration do not exhaust the definition of the acidic or

${ }^{6}$ L. Michaelis und A. Gyemant, Biochem. Zeitschr. 109, 166 (1920).-L. Michaelis und R. Krüger, Biochem. Zeitschr. 119, 307 (1921).

${ }^{7}$ N. Bjerrum, Die acidimetrische und alkalimetrische Titration. Stuttgart.

${ }^{8}$ L. J. Gillespie, Journ. of the Amer. Chem. Soc. 42, 742 (1920).

${ }^{9}$ C'f. Koppel und K. Spiro, Biochem. Zeitschr. 65, 409 (1914) 
basic nature of a solution. There is still another value which determines this nature and which can be best illustrated by the following example. Let us take a liter of normal acetic acid. According to data given on p. 40 its hydrion concentration is $\left[\mathrm{H}^{+}\right]=3.4 \times 10^{-3}$. Let us also take a liter of $3.4 \times 10^{-3} \mathrm{~N} \mathrm{HCl}$. The two solutions have the identical $\left[\mathrm{H}^{+}\right]$and yet very different equivalent acid content. But they also differ in another respect. If we add to each one cubic centimeter of a $3.4 \mathrm{~N} \mathrm{NaOH}$ solution the resulting relative changes in the $\left[\mathrm{H}^{+}\right]$of the two solutions will be widely different. The $\mathrm{HCl}$ solution has become neutral in reaction, while the acetic acid has remained still acid in reaction. The acetic acid solution, in spite of having an identical $\left[\mathrm{H}^{+}\right]$, presents a greater reserve of acid; it contains, as it were, latent or potential H-ions. On the other hand the $\mathrm{HCl}$ solution is less resistant to the addition of alkali in respect to the change of its $\left[\mathrm{H}^{+}\right]$. This resistance cannot be directly expressed in terms of the equivalent content of a solution. This can be seen from the following example. A solution of acetic acid in pure water on one hand and in a normal sodium acetate solution on the other hand have the same equivalent content of free acid. But if the same amount of $\mathrm{NaOH}$ solution be added to each, the $\left[\mathrm{H}^{+}\right]$of the pure acid solution will change to a much greater extent than in the acidacetate mixture. The pure acid solution yields more readily, the acid salt mixture changes its $\left[\mathrm{H}^{+}\right]$relatively much less. This resistance to change in reaction is a property of the greatest significance in physiology. The acids arising in the metabolic processes (carbonic acid, lactic acid) change the reaction of the tissue fluids. For the proper functioning of the organism it is necessary that the tissue fluids have a great enough buffering effect to prevent the increase in hydrogen ion concentration to a deleterious extent. On the other hand, this resistance must permit a great enough increase in the $\left[\mathrm{H}^{+}\right]$ to set in motion the automatic responses of the organism for the removal of these acids. This concept of resistance requires therefore a more precise definition which can be arrived at in the following manner:

If to a liter of the acid solution increasing amounts of $N \mathrm{NaOH}$ solution be gradually added, then for at least a very small period of this addition the change in $\mathrm{pH}$ will be proportional to the amount of alkali added. This applies at least to infinitely small amounts of added alkali. As the addition of alkali progresses, this proportionality 
disappears. Let us now designate a very small amount of added alkali by $\mathrm{dL}$, then $\frac{\mathrm{dL}}{\mathrm{dpH}}$ becomes the direct measure of the "buffer value" of the solution. Let us now calculate this value for certain cases. We can do this graphically by making use of the titration curves shown in figs. 12 and 13 page 99). Since in these curves $\mathrm{pH}$ is represented by the ordinates and $L$ by the abscissae, it follows that $\frac{\mathrm{dL}}{\mathrm{dpH}}$ at each point is equal to the cotangent of the angle of inclination of the curve. As it will be readily seen the buffer value is greater for every given amount of alkali with any acid the greater the equivalent concentration of free acid. If this concentration should approach zero then the buffer value also approaches zero. This will mean then that in very dilute acid solutions small amounts of alkali will effect very large changes in the $\mathrm{pH}$. But not only the concentration of free acid, but also the nature of the acid itself has an effect on the buffer value. In comparing the curve for $\mathrm{HCl}$ and for a weak acid, such as acetic, we observe only in the curve of the latter a flat or wide point of inflection indicating the formation of the maximum of the buffer value, while the minimum is shown by a steep point of inflection. The flat point of inflection or the maximum occurs at half of the equivalent neutralization, i.e., in the presence of equal concentrations of acetic acid and of sodium acetate.

This may be demonstrated analytically in the following manner: Let the total amount of acetic acid in the solution be designated by $\mathrm{S}$, the amount of added alkali by $\mathrm{L}$, then $\mathrm{S}-\mathrm{L}=$ the amount of free acetic acid and $\mathrm{L}=$ the amount of sodium acetate. Now in any mixture of acetic acid and sodium acetate we have

$$
\left[\mathrm{H}^{+}\right]=\mathrm{k} \frac{[\text { free acid }]}{[\text { Na acetate }]}=\mathrm{k} \frac{\mathrm{S}-\mathrm{L}}{\mathrm{L}}
$$

therefore, $\mathrm{pH}=-\log \mathrm{k}-\left(\frac{\mathrm{S}}{\mathrm{L}}-1\right)$ and the buffer value $\nu$

$$
\nu=\frac{d L}{d p H}=-\frac{d L}{d \log \left(\frac{S}{L}-1\right)}=\frac{L}{S} \cdot \frac{(S-L)}{\log e}
$$


The maximum of the buffer value is then established when

$$
\frac{d \nu}{d L}=\frac{(S-2 L)}{S \cdot \log e}=0
$$

or,

$$
L=\frac{S}{2}
$$

Therefore, it occur's in a mixture of equal concentrations of acetic acid and sodium acetate.

The numerical relation of the buffer value $\nu$ in a mixture of a weak acid and its salt may be obtained from equation (I) above:

$$
\nu \times \log \mathrm{e}=\frac{1}{\mathrm{~N}}=\frac{[\text { bound acid }]}{[\text { total acid] }} \times \text { free acid }
$$

If for the sake of simplicity we neglect the factor $\log$ e in the above calculation permitting a slight alteration in the definition, and assume $\nu \times \log$ e to be equal to the buffer value where $\nu$ has its former value of $\frac{d L}{d p H}$, then equation III gives us a very simple expression for the buffer value. This holds only for a mixture of an acid and its salt, and that only within the limits within which the approximation formula

$$
\left[\mathrm{H}^{+}\right]=\mathrm{k} \frac{[\text { free acid }]}{[\text { salt }]}
$$

is applicable. This range may be approximately estimated between the values $\mathrm{pH}=\mathrm{pk}+2$ and $\mathrm{pH}=\mathrm{pk}-2$. The reciprocal value $\frac{1}{\mathrm{~N}}$ may be designated as the "yielding power." 10

The dissociation constant of the acid does not enter into the expression III. Hence it follows that:

The "yielding power" and with it the buffering effect of an acid-salt mixture are independent of the nature of the acid. Thus the relatively strong acetic acid and the very weak boric acid when appropriately mixed with their respective sodium salts have the same buffering effect, except that the maximum of their buffer effects is at different $\mathrm{pH}$ values. This maximum may be evaluated in the following way:

10 "Nachgiebegkeit"-frequently used by the author in the sense of the reciprocal of buffer value.-Translator. 
From equation II we see that the maximum obtains in mixtures consisting of equivalent amounts of the acid and of the salt; but in such a mixture $\left[\mathrm{H}^{+}\right]=k$, the dissociation constant of the acid. The strength of the acid therefore influences only the $\mathrm{pH}$ range in which the mixture of the acid and its salt acts as a good buffer.

It should now be clear as to just what constitutes a poorly or a well buffered solution. The magnitude of the above defined buffer value is the sole measure for it. Thus distilled water and $\mathrm{NaCl}$ solutions are poorly buffered; river and sea waters are still relatively poorly buffered; much better in this respect is blood, and still better are the special buffer solutions prepared in the laboratory.

The first coherent and exhaustive exposition of these relations was given by Koppel and Spiro (l. c.) Their derivation is terminologically slightly different from the one given here, insofar as we have attempted to adhere closely to the derivations contained in the earlier chapters. Koppel and Spiro take also for their point of departure the conception that given two solutions of the same $\mathrm{pH}$, of which one is only a strong acid and the other a buffer mixture, the first will be more susceptible to the addition of base, as far as change in $\mathrm{pH}$ is concerned, than the second. They designated the action of the buffer as a "moderating" one, and the buffers themselves they named "moderators."

By the "yielding power" of a solution we have hitherto understood its behavior toward the addition of small amounts of either strong acid or base. But practically even of greater interest is the yielding power of a buffer solution to the addition of a small amount of the buffering acid itself. The commonest physiologically encountered buffer is the system $\mathrm{CO}_{2}+\mathrm{NaHCO}_{3}$. There is a constant formation in the metabolic processes of $\mathrm{CO}_{2}$, and the obvious question ariseswhat is the special buffer effect of such a carbonate buffer towards the addition of free $\mathrm{CO}_{2}$ ? In a mixture of a free weak acid in concentration $\mathrm{A}$ and of its alkali salt in concentration $\mathrm{S}$ we have the relation

$$
\left[\mathrm{H}^{+}\right]=\mathrm{k} \frac{\mathrm{A}}{\mathrm{S}}
$$

therefore,

$$
\mathrm{pH}=-\log \mathrm{k}-\log \mathrm{A}+\log \mathrm{S}
$$


Upon the addition of an amount $\mathrm{dA}$ of $\mathrm{CO}_{2}$ we have

$$
\frac{\mathrm{dpH}}{\mathrm{dA}}=-(\log \mathrm{e}) \frac{1}{\mathrm{~A}}
$$

By making use of the definitions given above, we may designate the ratio of the decrease of $\mathrm{CO}_{2}$ to the increase in $\mathrm{pH}$ as the "special buffer effect" of the mixture, $\nu^{\prime}$, which becomes simply $\nu^{\prime}=+\frac{1}{\mathrm{~A}}$, or, in terms of the reciprocal value, the "special buffer value," $\mathrm{N}^{\prime}$,

$$
\mathrm{N}^{\prime}=\mathrm{A}
$$

The "special buffer effect" of a buffer mixture consisting of $\mathrm{CO}_{2}$ $+\mathrm{NaHCO}_{3}$ is therefore independent of the concentration of the bicarbonate and is inversely proportional to the concentration of the carbon dioxide.

This remarkable law expresses a mode of defense of the living organism against changes in $\mathrm{pH}$ which might be caused by the increase of $\mathrm{CO}_{2}$. Augmentation of $\mathrm{CO}_{2}$ in the blood sets in motion the automatic processes which lead to the elimination of the $\mathrm{CO}_{2}$ present in excess. But should these automatic processes temporarily fail because of an excessive strain upon them, then the accumulation of $\mathrm{CO}_{2}$ in the blood results in a progressively decreasing change in $\mathrm{pH}$. It will be shown later that in acidosis there is a smaller concentration of free $\mathrm{CO}_{2}$ in the blood than normally. The "special buffer effect" of such blood is considerably below the normal value, or in other words, the acidotic blood is more poorly buffered than normal blood. The same amount of $\mathrm{CO}_{2}$ arising in the metabolism changes the $\mathrm{pH}$ of the acidotic blood more than it would the $\mathrm{pH}$ of normal blood. The irritation produced by a given amount of $\mathrm{CO}_{2}$ in all regulatory centers controlled by the $\mathrm{pH}$ of the blood, is greater in acidosis than in the normal state. ${ }^{11}$

11 Simultaneously with the publication of the second German edition of this book there appeared an article by Van Slyke (D. D. Van Slyke, Journ. Biol. Chem., 52, 525, (1922)) in which the theory of buffers was treated on the same basis but much more thoroughly. For further study of this problem this article is especially recommended. 


\section{CHAPTER III}

\section{The Dissociation of Strong Electrolytes}

\section{SUMMARY OF CONTENTS}

The strong electrolytes appear to deviate from the law of mass action as regards their dissociation equilibria. But this appears only because of an erroenous interpretation formerly given to conductivity measurements. The way in which the state of dissociation of strong electrolytes was calculated from these measurements can no longer withstand the criticism of our day. The clarification and correction of these earlier inaccuracies yielded the theory of activity. This theory is presented in a simple form in accord with the points of view developed in the literature, and which is also quite sufficient for the working purposes of the physiologist. The strong electrolytes are practically always completely dissociated, and, furthermore, if weak electrolytes be present along with them in solution, they appear to raise the dissociation constants of these weak electrolytes. In physiological processes it is not the absolute dissociation constants of weak electrolytes (such as $\mathrm{CO}_{2}$ ) but the constants reduced to the physiological salt content that are of significance.

\section{The deviations from the law of mass action and their significance}

Hitherto all of our considerations were based on the assumption that the "strong" electrolytes are always completely dissociated in solution and that they have no other influence on the ionic equilibrium apart from that defined by the mass law. These assumptions are particularly valid for very dilute solutions of the strong electrolytes. It is now our purpose to introduce certain corrections requisite for the higher concentrations. The great interest of this problem lies not only in the insignificant corrections of our earlier calculations, but also in the theoretic elucidation of the character of these deviations. These enigmatic deviations from the otherwise well accepted laws of physical chemistry are being gradually cleared 
up by careful investigations. ${ }^{1}$ Our presentation of this subject is but a preliminary attempt, and there is much in it that still awaits ultimate solution. The perusal of this section of our book then cannot yet yield the degree of satisfaction to be obtained from a well established chapter of a natural science.

The peculiarity of the strong electrolytes first became evident in those apparent deviations from the mass law which were found in their dissociation. For the purpose of introducing our problem let us consider the determination of the dissociation constant of $\mathrm{KCl}$, which by the mass law is defined as

$$
\mathrm{k}=\frac{[\mathrm{KCl}]}{\left[\mathrm{K}^{+}\right]\left[\mathrm{Cl}^{-}\right]}
$$

The method of estimating this constant $\mathrm{k}$ appears to be simple at first sight. All one needs is to determine the degree of dissociation in $\mathrm{KCl}$ solutions of various concentrations. If this is designated as $\alpha$, then

$$
\mathrm{k}=\frac{\alpha^{2}}{1-\alpha} \times \mathrm{c}
$$

where $\mathrm{c}$ is the total concentration $\mathrm{KCl}$, this being Ostwald's dilution law which has rendered such excellent service in the estimation of dissociation constants of the weak electrolytes. Of the different ways for the determination of the degree of dissociation the conductivity method was always considered the best. This method is based on the following assumptions: (1) The conductivity of a solution of an electrolyte is a purely additive function of the conductivities of its component ions. (2) Every individual ionic species possesses within wide limits a constant molar conductivity. The latter is proportional to the motility of the ionic species (assumed to be constant), i.e., the rate of migration imparted to an ion by the action of a homogeneous electric field of unit strength. From these assumptions we derive the following expression which serves as a measure of the degree of dissociation, $\alpha$,

$$
\alpha=\frac{\Lambda}{\Lambda_{\infty}}
$$

1 For the earlier accounts see K. Drucker, Anomalie der starken Electrolyte. Stuttgart, 1905. 
$\Lambda$ is here the molar conductivity of the electrolyte at the concentration c (i.e., the specific conductivity at concentration $\mathrm{c}$, divided by c) and $\Lambda_{\infty}$ is the limiting value of this molar conductivity for infinite dilution. For such infinite dilution the dissociation may be assumed to be complete, or $\alpha=1$. If this rule be applied to a weak electrolyte a value of $\mathrm{k}$ is obtained which is independent of the concentration, as has been shown a very great number of times, and as it is illustrated for acetic acid, as an example, in table 18.

TABLE 18

\begin{tabular}{c|c|c|c}
\hline$\frac{1}{\mathrm{c}}$ & $\boldsymbol{\Lambda}$ & $100 \times \alpha$ observed & caled. for $\mathrm{pK}=4.75$ \\
\cline { 1 - 2 } 0.994 & 1.27 & 0.402 & 0.42 \\
2.02 & 1.94 & 0.614 & 0.60 \\
15.9 & 5.26 & 1.66 & 1.67 \\
18.1 & 5.63 & 1.78 & 1.78 \\
$1,500$. & 46.6 & 14.7 & 15.0 \\
$3,010$. & 64.8 & 20.5 & 20.2 \\
$7,480$. & 95.1 & 30.1 & 30.5 \\
$15,000$. & 129. & 40.8 & 40.1 \\
\hline
\end{tabular}

TABLE 19

\begin{tabular}{|c|c|c|c|c|c|c|c|c|}
\hline \multirow{2}{*}{$\begin{array}{c}\text { Molar con- } \\
\text { centration } \\
\mathrm{c}\end{array}$} & \multicolumn{2}{|c|}{$\mathrm{IKCl}$} & \multicolumn{2}{|c|}{$\mathrm{HCl}^{*}$} & \multicolumn{2}{|c|}{ KOH† } & \multicolumn{2}{|c|}{ Na-acetate $\ddagger$} \\
\hline & $\alpha$ & $\mathbf{k}$ & $\alpha$ & $\mathrm{k}$ & $\alpha$ & k & $\alpha$ & $\mathrm{k}$ \\
\hline 0.001 & 0.973 & 0.043 & $(0.993)$ & $(0.14)$ & $(0.981)$ & $(0.06)$ & $(0.958)$ & 0.022 \\
\hline 0.01 & 0.941 & 0.16 & 0.972 & 0.34 & 0.917 & 0.10 & 0.894 & 0.077 \\
\hline 0.1 & 0.861 & 0.56 & 0.920 & 1.06 & 0.894 & 0.75 & 0.778 & 0.273 \\
\hline 1.0 & 0.755 & 2.3 & 0.791 & 3.0 & 0.771 & 2.6 & 0.525 & 0.58 \\
\hline 3.0 & 0.679 & 4.3 & 0.565 & 7.2 & 0.590 & 2.6 & 0.278 & 0.32 \\
\hline
\end{tabular}

Should we now attempt to apply the same method to $\mathrm{KCl}$ we should find that the value for $\mathrm{k}$ obtained will not be constant, but will vary in a definite direction. It will definitely increase with the concentrations of the salt. The same is true for all strong electrolytes such as $\mathrm{HCl}, \mathrm{HBr}, \mathrm{HI}, \mathrm{NaOH}, \mathrm{KOH}$, as well as for all salts, and even for such salts as ammonium acetate. Definite indications of this relationship are already evinced by the strongest of the weaker electrolytes, such as picric acid, tartaric acid, etc. Table 19 illus- 
trates this point for $\mathrm{KCl}, \mathrm{HCl}, \mathrm{KOH}$ and $\mathrm{Na}$-acetate, calculated from the conductivity measurements of Kohlrausch and his coworkers.

It has been attempted purely empirically to define the value of $\mathrm{k}$ in different ways, and we have an empirical expression given by van't Hoff: as well as one by Rudolphi. ${ }^{3}$ Van't Hoff's formula is

$$
\frac{\alpha^{3} \times \mathrm{c}}{(1-\alpha)^{2}}=\mathrm{k}
$$

and Rudolphi's

$$
\frac{\alpha^{2} \sqrt{\mathrm{c}}}{(1-\alpha)}=\mathrm{k}
$$

Both are interpolation formulae that are practically usable.

Arrhenius $^{4}$ had already attempted an explanation of these findings. $\mathrm{He}$ assumed that because of the contraction of the volume of water ("electrostriction") accompanying the process of solution of salts, alterations occurred in the dielectric constant, the osmotic pressure and the motility of the ions. As a consequence of this the dielectric constant and hence the dissociating capacity of the solvent upon the electrolyte are increased with the increasing concentration of the electrolyte which is being dissolved. In order to demonstrate this idea Arrhenius showed that the dissociation constant of a weak electrolyte (acetic acid) increases when a strong electrolyte $(\mathrm{NaCl})$ is, added to its solution. Thus the inverting capacity of acetic acid for cane sugar was also materially increased by the addition of salt. After allowing for that portion of the effect which he ascribed to the purely catalytic effect of the salt, he observed a still further acceleration of the catalysis by the salt, which he ascribed to the increased dissociation of the acetic acid effected by the presence of the salt. But even without such elaborate presentations, the foresight of theoretical chemists has long recognized that this contradiction to the mass law is only an apparent one. As Nernst puts it in his textbook: "it is highly probable, although on still unknown grounds, that neither the electric conductivity nor the osmotic pressure (lowering of the freezing point) give us an accurate scale for measuring the degree of dissociation."

2 van't Hoff, Zeitschr. f. physikal. Chem. 18, 300 (1895).

${ }^{3}$ C. Rudolphi, Zeitschr. f. physikal. Chem. 17, 385 (1895).

4 Sv. Arrhenius, Zeitschr. f. physikal. Chem. 31, 198 (1S99). 
A second method of estimating the degree of dissociation is by means of determining the lowering of the freezing point. From the latter one may calculate the molar concentration of the solution, and this may be related to that molar concentration which is to be expected by assuming a total lack of dissociation. If the latter concentration is $\mathbf{c}$ and the former $\mathrm{c} \times \mathrm{i}$ (i being "van't Hoff's coefficient"), then with binary electrolytes $\mathrm{i}-1=\alpha$. It is conspicuous that values for $\alpha$ obtained by this method do not agree exactly with those obtained by the conductivity method.

But there is still another method for the estimation of $\alpha$, namely, the concentration chain method. This method is applicable to a large series of electrolytes. By means of concentration chains one can measure the concentration of $\mathrm{H}^{+}, \mathrm{OH}^{-}, \mathrm{Cl}^{-}$and some other ions and hence determine the degree of dissociation of many acids, such as $\mathrm{HCl}, \mathrm{HNO}_{3}$ and of salts, such as $\mathrm{KCl}, \mathrm{NaCl}$, etc. Accurate investigations by this method have been made but recently ${ }^{5}$ when the technic has been considerably improved. . Bjerrum ${ }^{5 a}$ called attention to the fact that the values of $\alpha$ yielded by the three methods did not agree. Table 20 taken from Bjerrum shows some of the evidence. The apparent degree of dissociation obtained from the freezing point method, $\mathrm{f}_{\mathrm{o}}$, is designated as the osmotic factor; analogously $\mathrm{f} \mu$ is the conductivity factor; and that determined by the concentration chain as the "activity factor," $\mathrm{f}_{\mathrm{a}}$. As is seen from table 20, at the lowest concentrations the deviations do not appreciably exceed the limits of experimental error, but at the higher concentrations they do exceed it beyond all doubt. Since these different methods give series of three different results, it is justifiable to entertain doubts as to the reliability of all the three, or to doubt whether these three methods of measurement yield actually degree of dissociation figures.

The key to the solution of this enigma will be found in the following considerations which are based on the investigations of Bjerrum, ${ }^{5 \mathrm{a}}$ Milner ${ }^{9}$ and of Ghosh. ${ }^{8}$

The usual formula for the law of mass action is generally

${ }^{5}$ It must be mentioned that O. Jahn (Zeitschr. f. physikal. Chem. 33, 545 and 35, 1 (1900)) had long since observed a lack of agreement between degree of dissociation values of strong electrolytes calculated from conductivity and eoncentration chain measurements. He also recognized that the conductivity data were misleading, while he attached full value to his calculations from the concentration chain data.

sa N. Bjerrum Zts. Elektrochem. 24, 321, 1918. 
stated in terms of the concentrations of the reacting molecular or ionic species. This is justifiable only insofar as the concentration is proportional to the active mass. In reactions in gascous systems or in reactions between electroneutral molecules in solution the concept of the active mass may be quite accurately defined. In fact, it is the value which is proportional to the osmotic partial pressure of the molecular species involved. Actually the law of mass action, as it is thermodynamically conceived, makes no mention whatever of any concentration relationships but refers to pressures. ${ }^{6}$ Since under conditions of ideal gases and of extremely dilute solutions the osmotic pressure of a molecular species is proportional to its concentration, therefore it is permissible, for electroneutral molecules in very dilute solutions, to substitute concentration relations for those of osmotic pressure. For higher concentrations, however, this does not hold

TABLE 20

Apparent degree of dissociation of $\mathrm{KCl}$

\begin{tabular}{l|c|c|c}
\hline Molar concentration & $\mathrm{f}_{\mathrm{o}}$ & $\mathrm{f}_{\mu}$ & $\mathrm{f}_{\mathrm{a}}$ \\
\cline { 1 - 3 } 0.001 & 0.985 & 0.979 & 0.943 \\
0.01 & 0.969 & 0.941 & 0.882 \\
0.1 & 0.932 & 0.861 & 0.762 \\
1.0 & 0.854 & 0.755 & 0.558 \\
\hline
\end{tabular}

true any longer. Only with nonelectric molecular species (e.g., sucrose) concentrations up to $1 M$ may be considered in this respect, with very little error, as solutions of infinite dilution. In more concentrated solutions the osmotic pressure is affected by the forces of attraction operating among the molecules, and because of these the pressure is no longer proportional to the concentration but increases at a lesser rate than the concentration. It has been for a while supposed that with ionized substances the "state of infinite dilution" was to be assumed up to the same concentrations as with electroneutral molecules. The impossibility of determining experimentally the concentration of an individual ionic species strengthened this tacit assumption. But this is just where the error is involved. If in the case of electroneutral molecules the effect of the forces of attraction becomes evident in $1 M$ concentrations, then in the case of

-At one time Arrhenius spoke of it on this account as of the "pressure action law" in distinction from the "mass action law." 
ions with their free charges this should happen at much greater dilution. Furthermore, it cannot possibly be true that the conductivity of a concentrated electrolyte solution be simply an additive function composed of the sum of the conductivities of its ions, as it is in the case of very dilute solutions, for it is impossible to conceive that ions in a concentrated state could pass in the proximity of each other without producing a variety of force effects. Hence it follows that conductivity measurements made in concentrated solutions of ions cannot give us an accurate measure of the degree of dissociation; in the case of strong electrolytes at least they must simulate too low a degree of dissociation. Since this apparent degree of dissociation value is always large, i.e., very little less than 1 , the above mentioned authors proposed the hypothesis to the effect that the so-called strong electrolytes are always completely dissociated.

This hypothesis cannot as yet be proven as being absolutely exact. It is possibly analogous to such an acknowledged theory as, for example, the one asserting that in the solid crystallized state $\mathrm{KCl}$ does not exist in undissociated molecules but only as $\mathrm{K}^{+}$and $\mathrm{Cl}^{-}$ ions definitely spatially arranged. But if instead of "totally" we should say "almost totally" or "practically totally" dissociated, then we would at all events approach the true state of the matter much closer than did the older assumptions. We shall assume then with Bjerrum that in a $1 \mathrm{~N} \mathrm{KCl}$ solution, for example, the molecular species $\mathrm{KCl}$ is practically non-existent, and that each of the $\mathrm{K}^{+}$and $\mathrm{Cl}$ - ions are present in a $1 M$ concentration. But the active mass of the $\mathrm{K}^{+}$or $\mathrm{Cl}^{-}$ions in a $1 \mathrm{~N} \mathrm{FCl}$ solution is to be taken, however less than 1000 times that in a $0.001 \mathrm{~N}$ solution.

Bjerrum was led to the establishment of his hypothesis of the total dissociation of strong electrolytes by his observation that the absorption of light by solutions of colored strong electrolytes was independent of concentration and of other factors, provided there is no formation of complex ions. The chromium salts afforded convenient experimental material, because in solutions of these the formation of complex salts occurs very slowly, and it is possible for a time to work with complex-free solutions, which is not so easy with such other metal salts as those of iron. It was found that the light absorption of a gram-molecule of any chromium salt was quite independent of the concentration of its solution and of the acid anion with which it was combined. Since in all weak colored electrolytes (indicators) changes 
in the state of ionization are accompanied by changes in color, Bjerrum concluded that in the case of the chromium salts the state of dissociation did not change with dilution, in contradiction to the results apparently indicated by the molecular lowering of the freezing point. Of the possibilities of regarding chromium salts either as always undissociated or as always completely dissociated he chose the latter. It is the more probable assumption, because on the basis of our present day knowledge of the electric conductivity of salts, the former possibility is not acceptable, and because it agrees much better with the results deduced from the lowering of the freezing point methods.

This hypothesis aids us to orient ourselves, at least in a preliminary way, concerning the concentration of the ions in solutions of strong electrolytes. The problem still before us is to obtain quantitative relations also of conductivity, osmotic pressure and the active mass.

\section{The influence of ionic charge on the conductivity}

As long ago as $1912 \mathrm{Hertz}^{7}$ investigated the effect of the interionic electric forces upon the conductivity of the ions, and his results indicated that with increasing concentration the conductivity of the ions must decrease, even when no change in the degree of dissociation takes place. Since quantitative values which are not accurately known entered into his calculations, such as the mean free path of the ions, it must remain for future investigations to test his calculations. But in any case, qualitatively, Hertz's assertion is incontrovertible that in a mixture of positive and negative ions present in higher concentrations the total conductivity is not simply the algebraic sum of the conductivities of the separate ionic species, as it is in the case of the most dilute solutions. Consequently it is doubtlessly also incorrect to interpret the $\Lambda: \Lambda_{\infty}$ ratio as the "degree of dissociation."

I. Chandra Ghosh ${ }^{8}$ has recently proposed a promising way of calculating the conductivity of strong electrolytes. His point of departure was to the effect that the interionic potential occurring in electrolyte solutions does not disappear (see following section). Let A denote the "autopotential" per mol of the electrostatic re-

7 J. Hertz, Ann. d. Physik (4) 37, 1 (1912).

${ }^{8}$ I. Chandra Ghosh, Transact. Chem. Soc. London, 113, 449, 627, 707, 790 (1918); Zeitschr. f. physikal. Chem. 28, 211 (1921); see also: H. Kallmann, Zeitschr. f. physikal. Chem. 98, 433 (1921). 
ciprocal effect in an ionized solution of a completely dissociated electrolyte, whose molecule yields $\mathrm{n}$ ions. Then the electric conductance of this solution is such as if only the fraction $\mathrm{n} \times \mathrm{e}^{-\mathrm{A} / \mathrm{nRT}}$ of the mol were freely motile, while the remainder of the mol did not participate in the conduction of the current. This latter part of the mol is the one which, according to the earlier conceptions, was considered as the undissociated molecule. But according to Ghosh the electrolyte is completely ionized, just as according to the modern theory of structure of crystals, a salt in its solid crystallized state is totally ionized. Only at this juncture a deviation from the ordinary conception of an ion slips in, as it were. From the very beginning of the theory of electrolytic dissociation the term ion stood for a charged molecule (or "half-molecule") which moves under the influence of the electric current, but which was not originally split off from the uncharged molecule by the action of the current. Thus two properties had to be interrelated in order to characterize the ion: the free charge and the free motion of the particles. After it had been recognized that even in the crystal only these charged "half-molecules" and no salt-molecules proper were present, these were likewise called ions, although they lack the second characteristic, the free motility. Therefore, in accord with the definition of an ion, we shall either say: a dissolved electrolyte is always completely dissociated into ions, but only a fraction of it is freely motile; or: a dissolved electrolyte is always only partially dissociated into ions. It does appear, however, that the first mentioned, the newer conception of Ghosh, will become the dominant one in the future. It is even very possible that this conception is valid not only for the strong electrolytes, but for all electrolytes in general. If this is true, then a weak acid becomes under all conditions what we designated earlier as a "pseudo-acid" (see page 37 ), which exists in two tautomeric forms, of which one is not dissociated at all and the other completely dissociated.

The value $\alpha=\frac{\Lambda}{\Lambda_{\infty}}$, when it is taken not as denoting the degree of dissociation but rather in the sense of Ghosh as expressing the effect of the autopotential $A$, is calculated as $\mathrm{e}^{-\mathrm{A} / \mathrm{nRT}}$, or we can state it as

$$
\mathrm{A}=-\mathrm{nRT} \ln \alpha
$$


If the electrolyte solution be diluted, then the mean ionic distance is altered and consequently also the autopotential changed. Therefore, at infinite dilution an amount of electric work is performed to the extent of $\frac{\mathrm{e}^{2}}{\mathrm{Dr}}$, where $\mathrm{e}$ is the electric elementary quantum (the charge on one ion), $\mathrm{D}$ the dielectric constant and $\mathrm{r}$ is the original mean ionic distance. Furthermore $r=\sqrt[3]{\frac{\mathrm{V}}{2 \mathrm{~N}_{0}}}$ (where $\mathrm{N}_{0}$ is the number of molecules per liter, and $\mathrm{V}$ the volume of the solution), hence

$$
A=\frac{N_{0} e^{2} \sqrt[3]{2 N_{0}}}{D \sqrt[3]{V}}
$$

and therefore,

$$
\frac{N_{o} e^{2} \sqrt[3]{2 N_{o}}}{D \sqrt{V}}=2 R T \ln \alpha .
$$

From the last expression the value of $\alpha$ may be calculated, and then one finds good agreement between observed and calculated values of $\alpha$ for a large number of strong electrolytes, provided it is assumed at the same time that their dissociation is complete at each concentration used.

\section{The effect of the ionic charge upon the lowering of the freezing point}

In two excellent papers Milner ${ }^{9}$ shows that in a mixture containing equal numbers of positive and negative ions the electrostatic forces exercise a characteristic effect upon the average spatial distribution of the ions. Milner applied a theorem proposed by Boltzman: when a large number of molecules is clistributed in a defined space, then any two neighboring molecules in a given period of time are separated by varying distances for which a quite definite statistical mean can be determined. When these molecules are conceived to be ions, and, furthermore, when the numbers of positive and negative ions are equal, then we find that the distance between any two neighboring ions of the same charge, which always repel each other,

${ }^{9}$ S. R. Milner, The Virial of a Mixture of Ions. Phil. Mag. 23, II, 551 (1912); The Effect of Interionic Forces on the Osmotic Pressure of Electrolytes. Ibid. 25, II, 742 (1913). 
is on the average greater than the mean distance between two oppositely charged ions which attract each other. Between two univalent ions there is a potential difference which may be expressed as $\frac{\mathrm{e}^{2}}{\mathrm{r}}$, where $\mathrm{e}$ is the charge on the ion and $\mathrm{r}$ is the distance. The potential between two ions of the same charge is reverse in sign to that existing between two ions oppositely charged, which is due to repulsion in the first and to attraction in the latter case. If the mean distance between any two neighboring identically charged ions were equal to that between two oppositely charged ions, then the algebraic sum of the individual potential differences would be equal to zero.

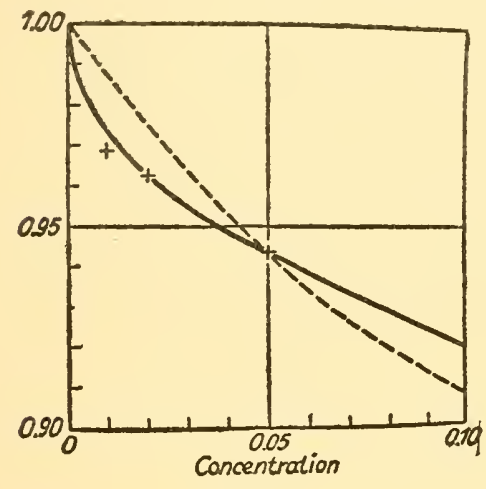

FIG. 15

(Taken from Bjerrum)

But since these distances are not equal, therefore the total potential is not $=0$, and as a result there is an average potential difference, in the sense that the attraction exceeds the repulsion. In such a solution, therefore, there is stored a certain amount of potential difference. This deduction is used by Milner in his second paper to calculate the effect of the ionic charge upon the lowering of the freezing point of a solution. As a result of very complicated calculations he obtained the curves shown in figure

15. This figure shows in the dotted curve how the molar lowering of the freezing point should change with the concentration of an electrolyte consisting of two univalent ions, assuming the usual mass action law; while the solid line curve represents the results of Milner's calculations. The crosses indicate the values for $\mathrm{KCl}$ found by freezing point determinations. With the exception of the lowest concentrations for which the experimental technic is uncertain and the highest concentrations for which the accuracy of the calculations becomes uncertain, the observed points agree quite well with the calculated values. Bjerrum called the mutual effect of the ions in regard to their osmotic pressure "the Milner effect." It is, therefore, unnecessary to assume any measurably incomplete dissociation of $\mathrm{KCl}$ in order to explain the course of its lowering of the freezing point. 
33. The three deviation-coefficients, $\mathrm{f}_{0}, \mathrm{f}_{\mu}, \mathrm{f}_{\mathrm{a}}$, according to Bjerrum

In order to calculate the relative osmotic pressure of an ion from its concentration, c (assuming the osmotic pressure of a solution which approaches in character an ideal gas to be equal to 1) the concentration must be multiplied by a factor $\mathrm{f}_{\mathrm{o}}$, the osmotic coefficient, which is always less than 1 . The relative conductivity is similarly obtained (assuming the condictivity when it is not affected by electrostatic forces to be equal to 1 ) by multiplying by the factor $\mathrm{f}_{\mu}$, the conductivity coefficient. Also, the active mass is obtained by multiplying the concentration by the factor $\mathrm{f}_{\mathrm{n}}$, the activity coefficient.

In the preceding sections it has been attempted to attribute a further significance to the coefficients, $\mathrm{f}_{\mu}$ and $\mathrm{f}_{o}$, and now we come to the activity coefficient, which is the most significant for our purposes. We shall first outline briefly Bjerrum's attempt ${ }^{5 a}$ to derive theoretically $f_{\mathrm{a}}$ from $\mathrm{f}_{\mathrm{o}}$, and then the procedure for the practical determination of $f_{a}$.

1. According to Bjerrum's thermodynamic derivation the following relationship exists between the osmotic factor $\mathrm{f}_{\mathrm{o}}$, determined by the freezing point method, and the activity factor $f_{a}$ and also the concentration $\mathrm{c}$ :

$$
f_{o}+\frac{c \cdot d f_{o}}{d c}=1+\frac{\operatorname{dlnf}_{a}}{d c}
$$

which is to be taken by all means only as an approximation formula for dilute solutions. From this, therefore, one can calculate $f_{a}$ from $f_{o}$, at least under certain simple conditions, and in that particular case when the osmotic coefficient is given with sufficient accuracy by the freezing point method.

Instead of the above complicated function Bjerrum has also worked out a simpler approximate method for the calculation of the activity coefficient in a solution of a strong electrolyte. Thus for a solution of a strong electrolyte whose molar concentration is $\mathrm{c}$ and which is composed of two equivalent ions (i.e., two univalent or two divalent etc. ions, as in the case of $\mathrm{KCl}, \mathrm{MgSO}_{4}$, but not $\mathrm{Na}_{2} \mathrm{SO}_{4}$ ) he obtained the expression

$$
-\log f_{a}=\frac{26}{D} n^{2} \sqrt[3]{c}
$$


where $\mathrm{D}$ is the dielectric constant of the solvent and $\mathrm{n}$ the valence of the ions. Therefore, for aqueous solutions it becomes

$$
-\log \mathrm{f}_{\mathrm{a}}=0.32 \cdot \mathrm{n}^{2} \sqrt[3]{\mathrm{c}}
$$

This formula is derived under certain assumptions from the one above (1a). But it is to be used only as an approximation for rough estimates. The factor 0.32 is in reality not quite accurately applicable to the different electrolytes; thus for $\mathrm{KCl}$ it appears, according to Bjerrum, that the factor 0.25 and for $\mathrm{HCl}$ the factor 0.2 are more exact. Let us now calculate for an example the value of $f_{a}$ for a $0.1 \mathrm{~N} \mathrm{KCl}$ solution, from the formula- $\log \mathrm{f}_{\mathrm{a}}=0.25 \mathrm{n}^{2} \sqrt[3]{\mathrm{c}}$ then $\mathrm{c}$ being given as 0.1 ,

$$
\begin{aligned}
-\log f_{a} & =0.25 \sqrt[3]{0.1}=0.25 \cdot 0.464=0.116 \\
\log f_{a} & =0.884-1 \\
f_{a} & =0.766
\end{aligned}
$$

This formula is quite serviceable for dilute solutions up to $0.1 \mathrm{~N}$. Besides, as stated above, the appropriate factor for $\sqrt[3]{\mathrm{c}}$ must be chosen for each kind of electrolyte. As for the practical application of this formula no more will be said for the present.

2. But the most accurate method for the determination of $f_{a}$ is the following: It is well established that in extremely dilute solutions $\mathrm{f}_{\mathrm{a}}=1$, i.e., the activity and concentration become identical. Without this assumption the concept of activity has no meaning. Now, if we set up a concentration chain with hydrogen electrodes and two $\mathrm{HCl}$ solutions of which one is extremely dilute $\left(\mathrm{c}_{1}\right)$ and the other of any conveniently chosen concentration $c_{2}$, then according to our above outlined consideration the electromotive force developed will not be

$$
\mathrm{E}=\frac{\mathrm{RT}}{\mathrm{F}} \ln \frac{\mathrm{c}_{1}}{\mathrm{c}_{2}}
$$

but

$$
\mathrm{E}=\frac{\mathrm{RT}}{\mathrm{F}} \ln \frac{\mathrm{c}_{1}}{\mathrm{c}_{2} \cdot \mathrm{f}_{\mathrm{a}}}
$$

For the very dilute solution of concentration $c_{1}$ we may omit the activity factor, for it is here $=1$, Since the values of $c_{1}, c_{2}$ and $E$ are either known or measurable, $\mathrm{f}_{\mathrm{a}}$ may therefore be calculated from the 
above expression. All that is necessary is to choose the concentration $c_{1}$ so small that the activity factor could be assumed to be $=1$. Such determinations can be made for all those ions for which reversible electrodes are available, especially $\mathrm{H}^{+}, \mathrm{Cl}^{-}, \mathrm{OH}^{-}$; and electrolytes such as, for example, $\mathrm{HCl}, \mathrm{KOH}, \mathrm{KCl}, \mathrm{NaCl}$, etc. are appropriate for this purpose.

Thus Noyes and Mac Innes ${ }^{10}$ employing this method found the values given in table 21 for the activity factor.

TABLE 21

\begin{tabular}{|c|c|c|c|c|c|c|}
\hline \multirow{2}{*}{$\begin{array}{c}\text { Molar } \\
\text { concentration } \\
\text { c }\end{array}$} & \multirow{2}{*}{$-\log c$} & \multicolumn{4}{|c|}{$\mathrm{f}_{\mathrm{a}}$ Noyes und MacInnes } & \multirow{2}{*}{$\begin{array}{c}\mathrm{f}_{\mathrm{a}} \text { for } \mathrm{KCl} \\
\text { Bjerrum }\end{array}$} \\
\hline & & $\mathrm{KCl}$ & $\mathrm{LiCl}$ & $\mathrm{HCl}$ & KOII & \\
\hline 0.001 & 3.000 & 0.979 & 0.976 & & & 0.943 \\
\hline 0.003 & 2.523 & 0.943 & 0.945 & 0.990 & 0.982 & \\
\hline 0.005 & 2.301 & 0.924 & 0.930 & 0.965 & 0.975 & \\
\hline 0.010 & 2.000 & 0.890 & 0.905 & 0.932 & 0.961 & $0.8 \$ 2$ \\
\hline 0.030 & 1.523 & 0.823 & 0.848 & 0.880 & 0.920 & \\
\hline 0.050 & 1.301 & 0.790 & 0.817 & 0.855 & 0.891 & \\
\hline 0.100 & 1.000 & 0.745 & 0.779 & 0.823 & 0.816 & \\
\hline 0.200 & 0.699 & 0.700 & 0.750 & 0.796 & 0.79 .3 & 0.762 \\
\hline 0.300 & 0.523 & 0.673 & 0.738 & 0.783 & 0.769 & \\
\hline 0.500 & 0.301 & 0.638 & 0.731 & 0.773 & 0.765 & \\
\hline 0.700 & 0.155 & 0.618 & 0.734 & 0.798 & 0.772 & \\
\hline 1.0 & 0.000 & 0.593 & 0.752 & 0.829 & 0.786 & 0.558 \\
\hline 2.0 & -0.301 & - & - & 1.040 & - & \\
\hline 3.0 & -0.477 & - & 1.164 & 1.402 & - & \\
\hline
\end{tabular}

\section{The hydration of ions and its significance for the activity}

Bjerrum's approximation for the calculation of the activity factor may be applied with sufficient accuracy to electrolyte solutions of concentration up to about 0.2 molar, and, therefore, quite within the limits of concentration most frequently encountered in physiological investigations. But for higher concentrations it fails so completely that, for example, for a $3 \mathrm{~N}$ solution of $\mathrm{HCl}$ the activity factor was experimentally found to be $>1$ (see table 21), while theoretically this factor must decrease with increasing concentration. According to Bjerrum the cause for this discrepancy lies in the fact that the activity of the ions is influenced not only by the interionic electrostatic forces,

${ }^{10}$ Journ. Amer. Chem. Soc. 42, 239 (1920). 
but also by the degree of hydration of the ions ${ }^{11}$ which changes with the concentration. Only the essential features of this conception will be touched upon at this juncture.

There are many grounds for believing that there exists an affinity between the molecules of a dissolved substance and the solvent, and that the solvent is not merely the space in which the molecules are free to move. This affinity for the solvent becomes even more probable in the case of ions. At least two theories can be presented concerning the nature of this affinity.

In the first place, it may be assumed that each ion forms a hydrate stoichiometrically with a definite number of water molecules analogously to the formation of salts with water of crystallization. It can be conceived that in concentrated solutions with an insufficient excess of water the hydration of ions does not reach its maximum extent. But ions of different degrees of hydration cannot be considered equivalent from the standpoint of the mass law. It can further be conceived that only the few unhydrated ions that are present take part in the electromotive activity or in chemical reactions, or stated in other words, that the ions are first dehydrated when they become active. It is also conceivable that a very small number of water-free ions are in a state of equilibrium with the hydrated ions. Then it would follow that the active ionic mass is proportional to the concentration of the water-free ions. As long as the water is present in a large excess the ratio of water-free to hydrated ions will remain constant, and the active masses of the ions will be proportional to their respective concentrations. On the other hand, in concentrated solutions, this ratio is disturbed in the direction of the water-free ions, and the activity of the ions will be apparently too great. Bjerrum had even attempted to calculate the degree of hydration of ions from this deviation of the observed from the calculated activity values. In reality it can be easily observed from the preceding table how at the higher concentrations the factor $f_{a}$ exceeds the value 1 , which without these conceptions would be quite inexplicable.

The second possible theory would be based on the supposition that there are no hydrated ions formed in a stoichiometric way. Instead

${ }^{11}$ O. Sackur (Zeitschr. f. physiol. Chem. 70, 493 (1910)) had already made use of the theory of hydration in an attempt to explain the above discrepancy in the case of strong electrolytes. 
it may be assumed that there is a molecular attraction between every individual ion and all water molecules, which rapidly decreases with the distance from the water molecule. In this way we arrive at the idea that every ion is surrounded by a layer of closely adhering water molecules, and this in turn is surrounded by other layers of progressively less adhering water molecules. At the same time it is to be assumed that the reactivity of the ions (or their "activity") is diminished by being bound by water, and that this curtailment decreases in the presence of but little water.

Attempts to determine the hydration eapacity of different ions ${ }^{12}$ by various methods based on the first conception outlined above led to contradicting results. Thus Nernst gives the following figures for infinitely dilute solutions (taken from Riesenfeld):

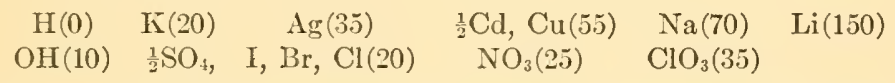

The figures in parentheses represent the numbers of bound $\mathrm{H}_{2} \mathrm{O}$ molecules. On the other hand Bjerrum found:

$$
\mathrm{H}(8) \quad \mathrm{Cl}(2) \quad \mathrm{K}(0)
$$

Thus the uncertainty concerning such figures is very great, and each of the many methods tried yielded different values. But at all events this uncertainty would seem to speak against the idea of stoichiometrically defined hydrates and it should be interpreted as being in favor of the theory of a general attraction existing between the ions and the surrounding water-molecules. ${ }^{13}$

On the other hand, almost all investigators agree on the serial sequence of the degree of hydration of the ions. Thus it is always observed that the hydration of the $\mathrm{H}$-ion is the least of all. With the alkali metal ions the degree of hydration decreases with increasing atomic weight in the manner.

$$
\mathrm{Li}>\mathrm{K}>\mathrm{Na}>\mathrm{Rb}>\mathrm{Cs}
$$

while with the halogen ions, reversely, it increases with increasing atomic weight, but not to so great an extent.

The obvious interpretation of this phenomenon is the following: The attraction for the water is evidently exercised by the free charge

${ }^{12}$ Nernst, Theoret. Chem. 7. Aufl. 1913, P. 413.

${ }^{13}$ K. Fajans, Naturwissenschaften 9, 729 (1921). 
of the ion. In the cations the free charge is located on the atomic nucleus. The greater the atomic radius the greater the distance from the unbound water-molecules and the smaller is the attraction. On the other hand, in the anions the free charge is on the periphery in the form of the valence electron, and its attraction for the water is but slightly influenced by the size of the atomic radius insofar as the greater or smaller distance of the positive nucleus weakens more or less the electric field of the electron. The attraction between the ions and the water had been conceived as an electrostatic one. A water-molecule is to be considered as being electroneutral when viewed remotely, so to speak, but more closely considered, it has electropositive as well as electronegative places, and positive ions are attracted to the negative places and conversely. Therefore, it may be readily imagined that each ion is first surrounded by a spherical layer of water-molecules and then by a second and perhaps by a third layer which are progressively less closely attached. The number of water-molecules which are firmly held in the inner layer depends upon spatial possibilities, much in the manner of Werner's coördination number. The hydrogen ion, in spite of its free positive charge, can, because of its small atomic radius, bind less water than any other ion.

With these intimations we must end our discussion of the deviations in concentrated solutions from Bjerrum's theory, especially in view of the incomplete state of knowledge of the subject and of the limited scope of this book. ${ }^{13 a}$

We see then that the underlying basis of the activity theory is beset with many difficulties. Nevertheless it leads us to an important and fundamentally quite well established result. It is only

${ }_{13 \mathrm{a}}$ The activity theory has been recently greatly advanced and developed in the experimental work of $\mathrm{G}$. N. Lewis and his collaborators, the theoretical studies of N. Bjerrum, and particularly through the fundamental work by Debye and Hückel. These developments have been so wide in scope that they can scarcely be summarized in a supplement. For an extensive study of these the reader is referred to G. N. Lewis and M. Randall, Thermodynamics, N. Y., 1923, wherein the methods of determination of activity coefficients as well as their thermodynamic derivation are particularly well given; also to the excellent summary of Debye's theories in E. Hückel, Zur Theorie der Electrolyte in Ergebn. d. exact. naturwiss., Vol. 3, Berlin, 1924, both of which books presuppose a good knowledge of electrophysics and of thermodynamics. 
because of this that the preceding theoretical observations were made. The result is the following.

\section{The significance of the activity theory for the $\mathrm{pH}$ of buffers}

In a mixture of a free weak acid and of its sodium salt the relation (page 44) holds:

$$
\left[\mathrm{H}^{+}\right]=\mathrm{k} \frac{[\text { free acid }]}{[\mathrm{Na}-\mathrm{salt}]}
$$

where $\mathrm{k}$ is the dissociation constant of the acid, and assuming that: (1) the salt is completely dissociated, and (2) as it must be again emphasized, that the total concentration of electrolytes (i.e., ions) in the solution is sufficiently small, so that concentration and activity may be taken as being equal to each other.

As was demonstrated in the last chapters, the first assumption is quite valid for all practical purposes. The basis on which the incomplete dissociation of $\mathrm{Na}$-salts had been previously assumed has been shown to be untenable, and at least all Na-salts are much more strongly dissociated than it had been assumed; and even at that, in $0.1 N$ solutions for example, a 90 per cent dissociation had been assumed for these salts. And now we must provisionally extend it to 100 per cent.

But the second assumption is valid only for very dilute solutions and besides for buffer solutions free of neutral salts; and these are physiologically unimportant. In fluids which are of physiological interest we find much more frequently buffer solutions of the following type:

A small amount of free weak acid + a small amount of its Na-salt + a relatively large amount of neutral salt $(\mathrm{NaCl})$; for example, in the blood:

$\mathrm{CO}_{2}$ of the order of magnitude................... 0.002 molar $\mathrm{NaHCO}_{3}$ of the order of magnitude............. 0.02 molar $\mathrm{NaCl}$ of the order of magnitude................ 0.12 molar or in sea-water:

$\mathrm{CO}_{2}$ of the order of magnitude.................. 0.0002 molar Bicarbonates of the order of magnitude.......... 0.002 molar $\mathrm{NaCl}$ of the order of magnitude................ 0.6 molar 
or in the urine:

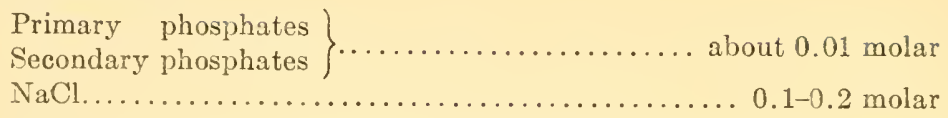

In the first portion of this book it has been stated that $\mathrm{NaCl}$ has no influence on the hydrion concentration of a solution and that equation (1) above remains unaffected. But at this juncture we must correct this statement. For we can no longer let the denominator in (1) stand for the concentration of the salt of the weak acid, or, what amounts to the same thing, for the concentration of the anions of this acid. What we now want is the activity of these anions. Equation (1) must now be restated as

$$
a_{\left[H^{+}\right]}=k \frac{[\text { free acid] }}{\text { Activity of the acid anions }}
$$

$\mathrm{a}_{\left[\mathrm{H}^{+}\right]}$is the activity of the $\mathrm{H}$-ions. The true concentration of the acid anions is known from the composition of the buffer and from the chemical analysis; it is $\mathrm{S}^{\prime}$ to retain our formerly used symbol. Then, activity of the acid anions $=\mathrm{f}_{\mathrm{a}} \times \mathrm{S}^{\prime}$.

The true concentration of the $\mathrm{H}$-ions is, on the other hand, entirely unknown. We have no direct method of measuring it, and probably it is of no great interest to us, for the effectiveness of the H-ions appears to depend only upon the $a_{\left[\mathrm{H}^{+}\right]}$, "the $\mathrm{H}$-ion activity." At this point therefore we shall agree to accept a change of significance of our symbols. From this point on the symbol $\left[\mathrm{H}^{+}\right]$will represent not the concentration but the activity of $H$-ions, and $p H$ its negative logarithm. Then equation (1) becomes:

$$
\left[\mathrm{H}^{+}\right]=\frac{\mathrm{k} \times[\text { free acid }]}{\mathrm{f}_{\mathrm{a}} \times[\text { salt of this acid }]}
$$

where the brackets still represent the concentration as usual.

What determines then the value of $\mathrm{f}_{\mathrm{a}}$ ? Since the Na-salt of the weak acid is present in but a small amount in comparison with the neutral salt $(\mathrm{NaCl})$, it is evident that this excess of the $\mathrm{NaCl}$ exercises almost the sole effect upon the activity of the buffer acid ions. The electrostatic forces to which each ion is subjected determine the activity coefficient; and since the ions of the $\mathrm{NaCl}$ are present in excess, they alone must determine the activity factor of the buffer acid ions. Therefore, when in a series of such solutions the amount of $\mathrm{NaCl}$ is kept constant, but always in excess, while that of the 
buffer acid is varied, then $\mathrm{f}_{\mathrm{a}}$ will remain for this particular buffer acid a constant value; and hence $f_{a}$ depends upon: 1 . the nature, and especially the valence, of the buffer acid, but not upon its concentration, as long as it remains relatively small, and 2. the kind, especially the valence, of the ions, and the concentration of the neutral salt in excess.

In equation (3) above we may designate

$$
\frac{\mathrm{k}}{\mathrm{f}_{\mathrm{a}}}=\mathrm{k}^{\prime}
$$

and then instead of (3) introduce as the corrected buffer equation the important equation

$$
\left[\mathrm{H}^{+}\right]=\mathrm{k}^{\prime} \frac{[\text { free acid }]}{[\mathrm{Na}-\text { salt of the acid }]}
$$

and the $\mathrm{k}^{\prime}$ which is substituted for the previous $\mathrm{k}$ we shall designate as the reduced dissociation constant of the acid, which has a well defined value for every $\mathrm{NaCl}$ concentration of the solution. $\mathrm{k}^{\prime}$ may be operated with as well as $k$. Since $k^{\prime}>k$, the entire effect of the influence of the neutral salt is such as to make it appear as if the dissociation constant of the acid were increased by it, i.e., as if the acid has become stronger.

\section{The reduced constants of physiologically important acids}

If we should wish to apply our buffer equation developed above to physiological problems, we must know not only the k-values for the $\mathrm{CO}_{2}$, phosphoric acid and similar buffers, but also their $\mathrm{k}^{\prime}$-values reduced to the corresponding content of sodium chloride and other neutral salts.

It has been experimentally confirmed that in a dilute buffer solution containing a constant amount of $\mathrm{NaCl}$ in excess $\mathrm{k}^{\prime}$ is a constant. Therefore it is entirely possible to render the field of experimental physiology entirely independent of the theory of activity; and all that is necessary for this purpose is to determine experimentally the $\mathrm{k}^{\prime}$ values for every physiologically important acid and for every significant concentration of neutral salt.

There are still very few exact data on this subject, and it is a pressing task for the immediate future to make available numerical data. Michaelis and Kruger ${ }^{14}$ determined the following $\mathrm{k}^{\prime}$ values for acetic acid.

${ }^{14}$ L. Michaelis and R. Krüger, Biochem. Zeitschr. 119, 307 (1921). 
These values for $\mathrm{pk}^{\prime}$ for acetic acid were determined electrometrically in mixtures each containing $0.02 N$ acetic acid +0.02 $\mathrm{Na}$-acetate and in addition $0.5 \mathrm{~N}$ of a neutral salt:

\begin{tabular}{l|l|l|l|l|l|l|l|l|l|l}
\hline & \multicolumn{8}{|c|}{ Neutral salt } \\
\hline $\mathrm{pk}^{\prime}$ of acetic acid... & 4.587 & $\frac{1}{2} \mathrm{~K}_{2} \mathrm{SO}_{4}$ & $\mathrm{KCl}$ & $\mathbf{\mathrm { KBr }}$ & $\mathrm{NH} \mathrm{H}_{4} \mathrm{Cl}$ & $\mathrm{NaCl}$ & $\mathrm{LiCl}$ & $\frac{1}{2} \mathrm{BaCl}_{2}$ & $\frac{1}{2} \mathrm{CaCl}_{2}$ \\
\hline
\end{tabular}

In a mixture of $0.1 \mathrm{~N} \mathrm{Na-acetate}$ with any concentration of acetic acid the value of $\mathrm{pk}^{\prime}$ was found to be 4.616 .

In a mixture of $0.02 \mathrm{~N}$ acetic acid $+0.02 \mathrm{~N} \mathrm{Na-acetate}$ but free of any other salt, $\mathrm{pk}^{\prime}=4.665$.

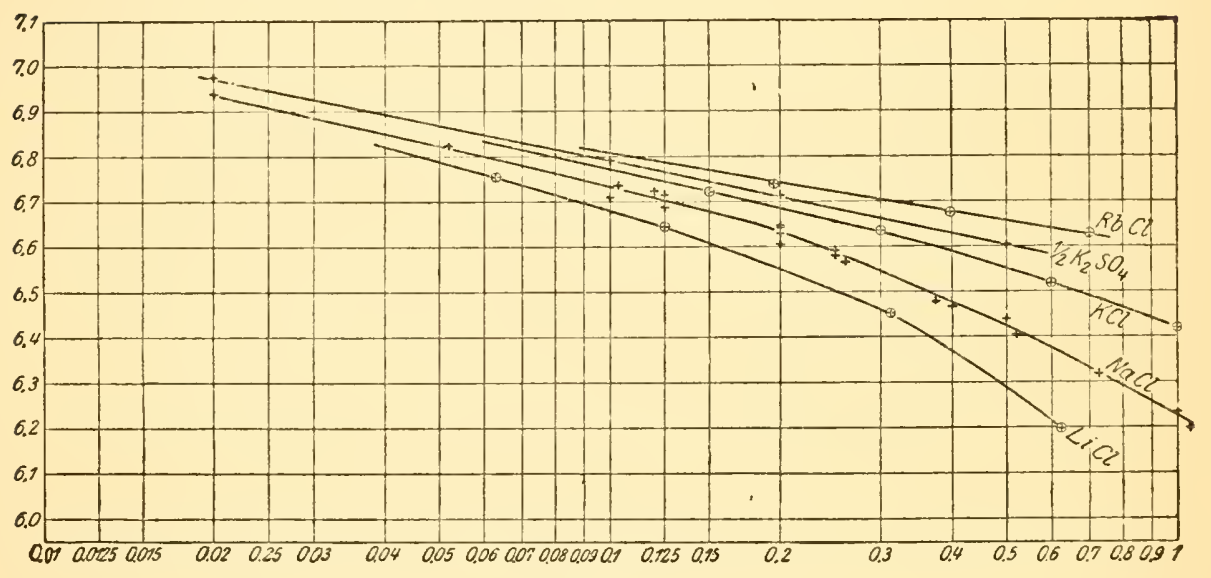

FIg. 16

For entirely salt-free solutions of acetic acid $\mathrm{pk}=4.733$ (see table, page 31 ).

These figures show that in the first place, $\mathrm{pk}^{\prime}$ of acetic acid in 0.5 $N \mathrm{NaCl}$ solution is 4.482 instead of 4.733 , as it is in a salt-free solution, and, secondly, that the effect varies with the different neutral salts.

For the second dissociation constant of phosphoric acid Michaelis and Kruger obtained $\mathrm{pk}^{\prime}$ values shown in figure 16 . The abscissa represent the neutral salt content of the solution in terms of normality, the ordinates are the $\mathrm{pk}^{\prime}$ values. The limiting $\mathrm{pk}^{\prime}$ value for infinitely small salt content, assymptotically approached by all the curves, is 7.10 .

It can be seen from these experiments with phosphoric acid that the value of $\mathrm{pk}^{\prime}$ is influenced not only by the concentration of the 
neutral salt (which alone occurs in Bjerrum's approximation equation for the activity factor), but is also greatly affected by its specific nature. Thus the influence of the alkali cations increases in the series $\mathrm{Rb} \mathrm{K} \mathrm{Na} \mathrm{Li}$; with anions it increases from $\frac{1}{2} \mathrm{SO}_{4}$ to $\mathrm{Cl} ; \mathrm{Br}$ appears to have the same effect as $\mathrm{Cl}$.

The most important for physiology is the reduced constant of carbonic acid, for it represents one of the most significant constants of nature. It will be dealt with in greater detail in a subsequent section.

With the change of $\mathrm{pk}^{\prime}$ accompanying variations of the saltcontent, the entire dissociation curve ( $\alpha$ - or $\rho$-curve) is simply displaced horizontally through the presence of the neutral salt. With the perception of this phenomenon the study of ionic equilibrium was greatly advanced, especially for the physiologists. ${ }^{14 a}$

\section{Another exposition on the effect of strong electrolytes ${ }^{15}$}

It appeared necessary in the presentation of this entire chapter to follow the historical development of this subject which is still in progress. But now we may attempt to find another method of representation, which comprises the physiologically important problems just as well, and which avoids many difficult complications.

The concentration of hydrogen ions is measured by means of the concentration chain. One solution whose unknown $\left[\mathrm{H}^{+}\right]=\mathrm{h}_{1}$ is in contact with a hydrogen-platinum electrode, and its potential is $\pi_{1}=0.058 \log \frac{h_{1}}{h_{0}}$, where $h_{0}$ denotes that hydrion concentration which the solution would have if $\pi=0$. The second solution of hydrion concentration $h_{2}$ is in contact with the second hydrogen-platinum electrode, and here the potential is $\pi_{2}=\log \frac{\mathrm{h}_{2}}{\mathrm{~h}_{\mathrm{o}}}$. It is usually assumed that the $h_{0}$ values in the first and second equations are identical, and hence the electromotive force $\mathrm{E}$ of the chain is

$$
\mathrm{E}=\pi_{1}-\pi_{2}=0.058\left(\log \frac{\mathrm{h}_{1}}{\mathrm{~h}_{0}}-\log \frac{\mathrm{h}_{2}}{\mathrm{~h}_{0}}\right)
$$

14 Concerning recent conceptions of the reduced constants see E. Warburg (Biochem. Journ. 16, 153, 1922).

15 This section is based upon the theory of concentration chains which is detailed in a later chapter. 
or

$$
\mathrm{E}=0.05 S \log \frac{\mathrm{h}_{1}}{\mathrm{~h}_{2}}
$$

$\mathrm{E}$ and $\mathrm{h}_{2}$ being known, $\mathrm{h}_{1}$ is easily obtained.

Criticism is invited when the question is raised as to whether it is justifiable to assume always that the two $h_{\circ}$ values are identical. If one of these solutions were aqueous and the other in anilin, it would never occur to anyone to regard the $h_{o}$ values as identical. But also in the case when both solutions are aqueous but the one is relatively poor and the other relatively rich in salt content, it is not correct to assume the same $h_{v}$ value for both. In such a case, as of a gas chain consisting of a $0.001 \mathrm{~N} \mathrm{HCl}$ on the one side and $0.001 \mathrm{~N}$ $\mathrm{HCl}+1 \mathrm{~N} \mathrm{KCl}$ on the other, we find, after allowing for the diffusionpotential, an E.M.F. of a few (2.5) millivolts. Previously, when it had been assumed that the degree of dissociation of the $\mathrm{HCl}$ in the two solutions was unequal, this E.M.F. was explained by the difference in the $\left[\mathrm{H}^{+}\right]$concentrations of the two solutions. But, as we learned above, this assumption rests on an erroneous interpretation, and we are no longer justified in accepting such an explanation. If we should actually suppose, as in the case above, that $h_{1}=h_{2}$ exactly, then the E.M.F. of such a chain can be explained only by assuming that the two $h_{0}$ values are unequal. If we designate this value as $h_{\circ}$ in the electrolyte-poor solution and $\mathrm{h}^{\prime}$ o in the electrolyte-rich solution, then the E.M.F. of this chain should rather be stated as

$$
\mathrm{E}=\pi_{1}-\pi_{2}=0.05 \mathrm{~s}\left(\log \frac{\mathrm{h}_{1}}{\mathrm{~h}_{0}}-\log \frac{\mathrm{h}_{2}}{\mathrm{~h}_{0^{\prime}}}\right)
$$

but since $h_{1}=h_{2}$,

$$
\mathrm{E}=0.058 \cdot \log \frac{\mathrm{h}_{0}{ }^{\prime}}{\mathrm{h}_{0}}
$$

Hence, we can express this condition by saying that: The electrolytic solution pressure of the $\mathrm{H}_{2}$ in an aqueous solution of low electrolyte content is somewhat different from that in an aqueous solution rich in electrolytes. And yet, when we apply, as we always do, the earlier incorrect formula for the calculation of the $h_{2}$ value from the E.M.F. of concentration chains, we obtain a purely calculated figure which cannot represent the actual concentration of the H-ions. But it is this calculated value which we designate as the result of our 
measurements and commonly accept as the desired $\left[\mathrm{H}^{+}\right]$- or $\mathrm{pH}$ value. An error was then made by using an incorrect formula for the calculation of the $\mathrm{H}$-ion concentration, and we designate this erroneously calculated concentration as the $\mathrm{H}$-ion activity.

And yet this calculated value appears to be of significance in the physical sense. In many cases in which $\mathrm{H}$-ions exercise any effect whatever the effect is proportional not to the true $\mathrm{H}$-ion concentration but apparently rather to their activity. Stated in other words: two solutions having an equal potential towards an $\mathrm{H}_{2}$-electrode frequently exhibit an equal effectiveness of their respective H-ions. The limits within which this provisional statement is valid must be established by future investigation, now that the underlying principles are to a certain extent clarified. In any event we have derived our justification to retain for the present, as a measure of the actual acidity, the values for $\left[\mathrm{H}^{+}\right]$and likewise $\mathrm{pH}$ calculated from the E.M.F. of the concentration chain in place of the true hydrion concentration.

AN EXAMPLE FOR THE DIFFERENT METHODS OF REPRESENTATION OF THE EFFECT OF STRONG ELECTROLYTES

It may be useful to clarify further the differences between the various explanations by means of an illustrative example. Let us take the concentration chain. ${ }^{16}$

$$
\mathrm{H}_{2}|0.001 \mathrm{~N} \mathrm{HCl}| \begin{gathered}
0.001 \mathrm{~N} \text { acetic acid } \\
+0.001 \mathrm{~N} \mathrm{Na} \text { - acetate }
\end{gathered} \mid \mathrm{H}_{2}
$$

In this case all the solutions are of such great dilution that in accord with any theory whatever the $\mathrm{HCl}$ and $\mathrm{Na}$-acetate may be assumed to be completely dissociated, and any possible effect of the ions upon the properties of the solvent may be neglected. We can, therefore, say that in the $0.001 \mathrm{~N} \mathrm{HCl}, \mathrm{h}_{1}=0.001 \mathrm{~N}$, and for the calculation of $h_{2}$ in the acetate mixture we may apply Nernst's formula (at $19^{\circ}$ )

$$
\mathrm{E}=0.579 \log \frac{\mathrm{h}_{1}}{\mathrm{~h}_{2}}
$$

${ }_{18}$ The two solutions in the actual arrangement are not in direct contact, but are connected by means of a saturated $\mathrm{KCl}$ solution, in order to avoid the diffusion potential which will otherwise arise. 
$\mathrm{E}$ being found to be at $19^{\circ} \mathrm{C}=0.0997$ volts, then by calculation $\mathrm{pH}=4.722$ and $\left[\mathrm{H}^{+}\right]=1.90 \times 10^{-5}$.

Now, the dissociation constant of acetic acid, as determined by the conductivity method, is $1.86 \times 10^{-5}$. From the buffer equation we should expect to find in the acetate mixture

$$
\left[\mathrm{H}^{+}\right]=\mathrm{k} \frac{[\text { Acetic acid }]}{[\mathrm{Na}-\text { acetate }]}=1.86 \times 10^{-5}
$$

which is in very good agreement with the above found value of 1.90 $\times 10^{-5}$. As long as the ionic content of our solutions does not exceed $0.001 \mathrm{~N}$, the theory, built upon the basis of the simple buffer equation, will yield a fairly complete picture of the relation of things.

Now let us take the concentration chain ${ }^{17}$

$$
\mathrm{H}_{2}|0.001 \mathrm{~N} \mathrm{HCl}| \begin{gathered}
0.1 \mathrm{~N} \text { acetic acid } \\
+0.1 \mathrm{~N} \mathrm{Na} \text { - acetate }
\end{gathered} \mid \mathrm{H}_{2}
$$

at $19^{\circ} \mathrm{C}$. it is found that $\mathrm{E}=0.0930$ volts. By applying again Nernst's formula we obtain for the acetate mixture $\mathrm{pH}=4.606$ and $\left[\mathrm{H}^{+}\right]=2.48 \times 10^{-5}$. How are we to explain then that in one equimolar mixture of acetic acid and Na-acetate we found $\left[\mathrm{H}^{+}\right]=1.90$ $\times 10^{-5}$ and in the other $\left[\mathrm{H}^{+}\right]=2.48 \times 10^{-5}$, according as to whether the concentration was $0.001 \mathrm{~N}$ or $0.1 \mathrm{~N}$ ?

1. The first older theory says: in $0.1 N$ solution Na-acetate is not totally dissociated, therefore, in this $\left[\mathrm{H}^{+}\right]$is not $=\mathrm{k} \frac{\text { acetic acid] }}{\text { [Na-acetate }]}$ but $=\frac{\mathrm{k} \text { [acetic acid }]}{\gamma \text { [Na-acetate }]}$, where $\gamma$ stands for the degree of dissociation of Na-acetate in $0.1 \mathrm{~N}$ solution. If we assume that the value of $\mathrm{k}\left(1.90 \times 10^{-5}\right)$ found in the first concentration chain to be correct, then $\gamma=\frac{1.90}{2.48}=0.766$. But as we have now come to believe, in reality $\gamma=1$, or very nearly so. Therefore, this interpretation is incorrect.

2. The activity theory says: Because of the Na-acetate content of the solution, the activity of the ions becomes different from their true concentration. The $\mathrm{Na}$-acetate is completely dissociated, there-

17 With same arrangement of chain as indicated in preceding footnote. 
fore, the concentration of acetate-ions, $\mathrm{C}_{\text {acetate }}=0.1 \mathrm{~N}$. The activity of the acetate ions $a_{\text {acetate }}=f_{a} \times C_{\text {acetate, }}$ where $f_{a}$, the activity factor, is $<1$. Furthermore, what is measured by means of the concentration chain is not the concentration of $\mathrm{H}$-ions, $\mathrm{C}_{\mathrm{H}^{+}}$, but their activity, $\mathrm{a}_{\mathrm{H}^{+}}$, and the relation between the two is

$$
\mathrm{a}_{\mathrm{H}^{+}}=\mathrm{f}_{\mathrm{a}}^{\prime} \times \mathrm{C}_{\mathrm{H}^{+}}
$$

where again ${f^{\prime}}_{a}<1$, but is not necessarily $=f_{a}$. The buffer equation must therefore be restated as

$$
a_{H^{+}}=\frac{k[\text { acetic acid }]}{\mathrm{f}_{\mathrm{a}^{\prime}}[\mathrm{Na}-\text { acetate }]}
$$

If we let $\mathrm{f}^{\prime}{ }_{a}=0.766$, then we obtain the desired value $\mathrm{a}_{\mathrm{II}^{+}}=2.48 \times$ $10^{-5}$. The concentration chain measurement does not yield anything directly concerning the value $\mathrm{C}_{\mathrm{H}^{+}}$, so long as there is no way of determining the value of $\mathfrak{f}_{a}^{\prime}$.

3. The third explanation says: As a solvent, the solution rich in salt content does not behave in the same way as pure water. Consequently, the solution pressure of the $\mathrm{H}_{2}-\mathrm{Pt}$ electrode towards it is not quite equal to that towards water. Hence the second of the above chains does not represent, strictly speaking, a concentration chain, for even two solutions of the same $\left[\mathrm{H}^{+}\right]$, one in salt-poor water, the other containing much salt, will give rise to an E.M.F. of a few volts. This adventitious E.M.F. must be subtracted from the observed E.M.F. of the second chain before we can utilize it as a true concentration chain for the calculation of $\mathrm{pH}$. The calculation carried out without this correction gives a value of $\mathrm{pH}$ which is in a certain respect too low, the calculated $\left[\mathrm{H}^{+}\right]$is hence too great by a definite factor.

Besides this, it is possible that in a solution containing much salt the dissociation constant of acetic acid is greater than in a salt-poor solution.

These two conditions bring about the result that $\mathrm{pH}$ calculated in the usual way has the value of only a calculation figure, which may deviate to the extent of about 0.1 from the original definition of $\mathrm{pH}$. But since the correction which we would have to introduce demands the knowledge of certain data which are not yet completely available, it is for the time being more practicable to define the uncorrected figure of our calculation as the $\mathrm{pH}$ value. 


\section{CHAPTER IV}

The State of Dissociation of Acids and Bases During Actual Salt Formation

SUMMARY OF CONTENTS

Not all salts are to be included with the strong, always totally dissociated electrolytes. In the presence of undissociated salt molecules there arise in the above developed dissociation laws complications which are discussed below.

38. Hitherto we based our considerations on the assumption that of all electrolytes only the weak acids and bases are incompletely dissociated, while all other electrolytes are completely dissociated. Doubtlessly this assumption of complete dissociation is but one of approximation which for such electrolytes as $\mathrm{KCl}$ must be practically quite exact. But there are also undissociated salt molecules. We are not as yet very well informed concerning them, but nevertheless the following facts are known:

In the first place it cannot be asserted in the case of solutions of organic salts in organic solvents that these salts are completely dissociated, which point will be taken up in greater detail in a later chapter. Secondly, we are not justified in ascribing, without further qualification, the properties of salts of the strong or moderately weak acids and bases to the salts of very weak acids and bases. If equivalent amounts of acetic acid and aniline are mixed in an aqueous solution, then this solution of aniline acetate differs from a $\mathrm{KCl}$ solution insofar as here a far reaching hydrolysis occurs. Secondly, we cannot, in this case, as we would with a $\mathrm{KCl}$ solution, deny the existence of the undissociated salt, aniline acetate. Many electrolyte effects would become more comprehensible, if such "actual" saltformation were assumed. It appears to the author that, for example, the explanation of electrolyte effect on protein solutions is not very adequate without the assumption of the formation of "actual" protein salts. Just which of the ionic species tend to form undissociated molecules cannot yet be accurately stated. It is only 
known with a fair degree of certainty that many of the compounds of $\mathrm{H}$ - and $\mathrm{OH}$-ions formed with oppositely charged ions (i.e., acids and bases) are but poorly dissociated. It seems probable that those ionic species which share with $\mathrm{H}$ - and $\mathrm{OH}$-ions the property of being strongly adsorbed on charcoal are also those which tend in the direction of true salt formation. The proteins bind the ions of organic dye-stuffs, of alkaloids, etc. It is out of the question that a salt of protein and methylene blue or quinine base belong to the type of always completely dissociated electrolytes. It must be even supposed that the same applies to protein salts of calcium ${ }^{1}$ and indeed to all protein salts. These intimations may suffice for the present to explain why the subject of true salt formation is brought into the discussion.

In an illustrative way our problem is as follows: When a base is added to acetic acid then the ensuing ionic equilibrium in the solution has been hitherto represented on the assumption that the molecular entity, "Na-acetate," was not at all formed. Let us now progress a step forward and assume that, after all, this molecular entity is formed, and indeed, according to the equation

$$
\mathrm{CH}_{3} \mathrm{COO}^{-}+\mathrm{Na}^{+} \rightleftharpoons \mathrm{CH}_{3} \mathrm{COO} \mathrm{Na}
$$

in the state of equilibrium

$$
\left[\mathrm{CH}_{3} \mathrm{COO}^{-}\right]\left[\mathrm{Na}^{+}\right]=\mathrm{k}_{\mathrm{s}}\left[\mathrm{CH}_{3} \mathrm{COO} \mathrm{Na}\right]
$$

where $\mathrm{k}_{\mathrm{s}}$ is the dissociation constant of the salt.

The advent of this molecular species affects the ionic equilibrium in the solution. Of the many problems arising out of this complication we shall limit ourselves to but a few. It is unessential, in this particular case of sodium acetate, that the formation of undissociated molecules is as yet scarcely considered as a practical possibility.

\section{The formation of the ionic equilibrium with true salt-formation ${ }^{2}$}

Let us take the following system. A very small amount of acid $\mathrm{A}$ is present in the concentration $\mathfrak{a}$ in solution. The concentration of its undissociated free molecules is a, its anions $\mathrm{A}^{-}$are in concentration $\mathrm{a}^{-}$, and besides the cation $\mathrm{H}^{+}$present in h-concentration there

${ }^{1}$ Cf. P. Rona and György, Biochem. Zeitschr. 56, 416 (1913).

${ }^{2}$ L. Michaelis, Biochem. Zeitschr. 103, 225 (1920). 
is present another cation $\mathrm{I}^{+}$in concentration $\mathrm{i}^{+}$which represents a sufficiently great excess so that the certain amount of salt formation from the interaction of the acid and $\mathrm{I}^{+}$does not lead to a material diminution in $\mathrm{I}^{+}$. In addition we must have for the maintenance of electroneutrality a corresponding amount of some other anions,

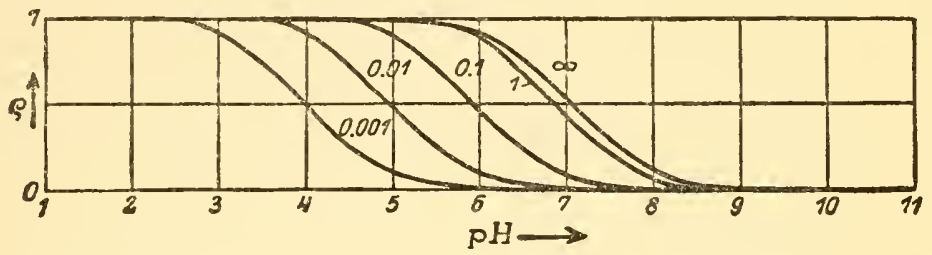

FIG. 17

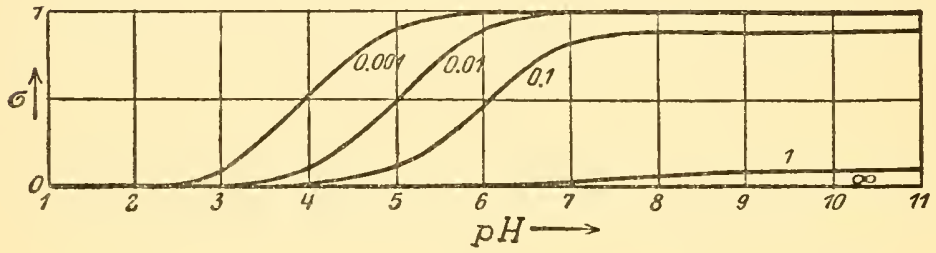

FIg. 1S

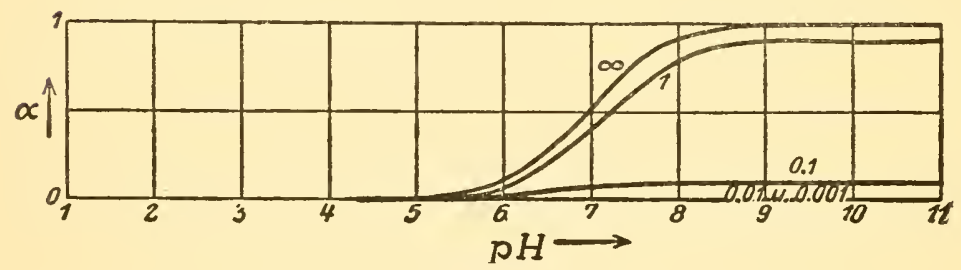

FIG. 19

Figs. 17 то 19. The functions $\rho, \sigma$, and $\alpha$ of an acid whose dissociation constant $\mathrm{k}_{\mathrm{a}}=10^{-7}$, in the presence of a neutral salt of an univalent cation in the concentration $0.1 \mathrm{~N}$, with the assumption that the acid and this cation form a salt, whose dissociation constant $\mathrm{k}_{\mathrm{g}}$ has the value marked on each curve.

which, however, exercise no effect upon the state of dissociation of the acid A. We shall now express the state of dissociation of this acid as a function of $h$. We define

The degree of dissociation $\alpha=\frac{\mathrm{a}^{-}}{\mathfrak{a}}$ 
The dissociation residue $\dot{\rho}=\frac{\mathrm{a}}{\mathfrak{a}}$

The degree of salt formation $\sigma=\frac{\mathrm{s}}{\mathrm{a}}$

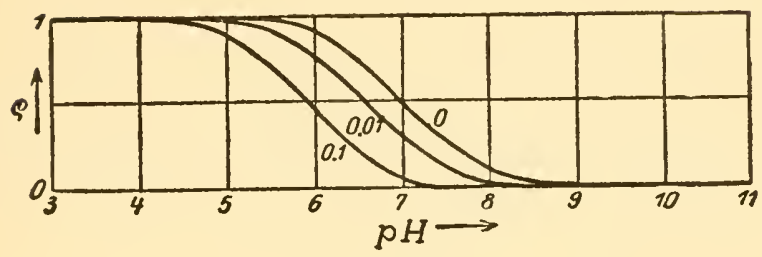

FIG. 20

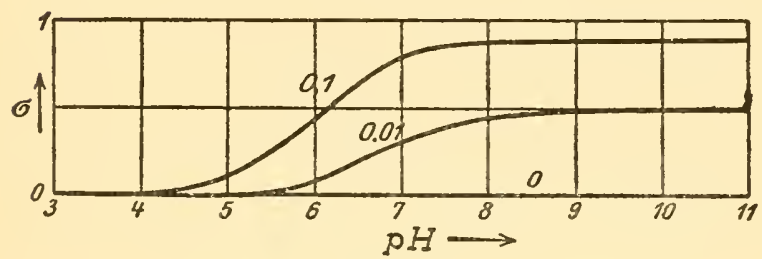

FIG. 21

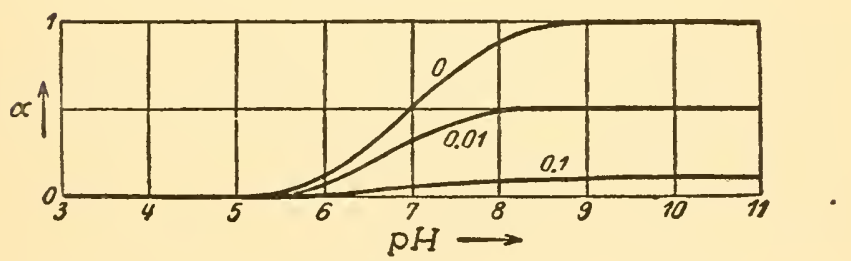

FIG. 22

Figs. 20 Tо 22. The functions $\rho, \sigma$, and $\alpha$ of an acid whose dissociation constant is $\mathrm{k}_{\mathrm{a}}=10^{-7}$, in the presence of a salt of an univalent cation in the concentrations marked on the curves, assuming that the acid and this cation form a salt whose dissociation constant $\mathrm{k}_{\mathbf{s}}=10^{-2}$.

where $\mathrm{s}$ is the concentration of the true salt formed by the acid $\mathrm{A}$ and the cation $\mathrm{I}^{+}$.

According to the definitions

$$
\mathfrak{a}=\mathbf{a}+\mathbf{a}^{-}+\mathbf{s}
$$


and the mass law demands that

$$
\begin{aligned}
& a^{-} h=k_{a} \times a \\
& a^{-} i^{+}=k_{a} \times s
\end{aligned}
$$

From these six equations we derive:

$$
\begin{aligned}
& \rho=\frac{1}{1+\frac{\mathrm{k}_{\mathrm{a}}}{\mathrm{h}}\left(\frac{\mathrm{i}^{+}}{\mathrm{k}_{\mathrm{g}}}+1\right)} \\
& \alpha=\frac{1}{1+\frac{\mathrm{h}}{\mathrm{k}_{\mathrm{a}}}+\frac{\mathrm{i}^{+}}{\mathrm{k}_{\mathrm{g}}}} \\
& \sigma=\frac{1}{1+\frac{\mathrm{k}_{\mathrm{s}}}{\mathrm{i}^{+}}\left(\frac{\mathrm{h}}{\mathrm{k}_{\mathrm{a}}}+1\right)}
\end{aligned}
$$

Of these functions that for $\rho$ is in the simplest form. In the absence of salt-formation its usual form is

$$
\rho=\frac{1}{1+\frac{k_{a}}{h}}
$$

The value $\mathrm{k}_{\mathrm{a}}$ in (Ia) is substituted in (I) by $\mathrm{k}_{\mathrm{a}}\left(\frac{\mathrm{i}^{+}}{\mathrm{k}_{\mathrm{s}}}+1\right)$, and this latter value is constant for a given concentration of added cations. This $\rho$-curve is therefore identical with that of any other acid whose dissociation constant amounts to $\mathrm{k}_{\mathrm{a}}\left(\frac{\mathrm{i}^{+}}{\mathrm{k}_{\mathrm{s}}}+1\right)$ without true salt formation. The meaning of this in other words is as follows: If we vary the $\mathrm{pH}$ of a very dilute acetic acid solution by the addition of a very small amount of $\mathrm{NaOH}$, then the $\rho$-curve has its originally derived form (page 000). But if this solution also contains an excess of a salt in constant concentration, then the resulting $\rho$-curve is such as it would be of an acid with a somewhat greater dissociation constant $\mathrm{k}_{\mathrm{a}}^{\prime}=\mathrm{k}_{\mathrm{a}}\left(\frac{\mathrm{i}^{+}}{\mathrm{k}_{\mathrm{s}}}+1\right)$. Graphically this is shown by a small horizontal displacement of the $\rho$-curve, this horizontal displacement is to the extent of $-\log \left(\frac{\mathrm{i}^{+}}{\mathrm{k}_{\mathrm{s}}}+1\right)$. This is shown in figure 17 for several assumed values of $\mathrm{k}_{\mathrm{s}}$, and in figure 20 for various values of $\mathrm{i}^{+}$ 
The $\alpha$-curve is here no longer an exact mirror image of the $\rho$-curve. The characteristic of this curve is that $\alpha$ reaches the value 1 more slowly, or, practically, does not reach it at all.

The $\sigma$-curve appears here for the first time. The curve of $\alpha+\sigma$ would be identical with the original $\alpha$-curve, i.e., it would be a mirror image of the $\rho$-curve. The $\sigma$-curve first becomes definitely marked at high $\mathrm{pH}$ values, that is to say, the true salt formation becomes first apparent at a certain degree of alkalinity. This condition can be restated as follows: The $\mathrm{H}$-ion and the other cation present compete for the combination with the acid. Only when there is a relative lack of $\mathrm{H}$-ions does the other cation succeed in the formation of a salt.

For the bases all these conditions are just as valid, after making the appropriate substitutions.

\section{The behavior of ampholytes in the process of true salt- formation}

The relations of an ampholyte to this process are more involved. We shall derive only the $\rho$-function.

We shall make the following designations:

1. $\mathfrak{a}$-the total concentration of the ampholyte.

2. $a^{\prime}$ and $a^{\prime}$-the respective concentrations of the ampholyte cations and anions.

3. a-the concentration of the free undissociated ampholyte molecules.

4. $\mathrm{s}_{\mathrm{I}}$ - the concentration of the salt formed by the ampholyte as an acid with a cation.

5. $s_{\text {II }}$-the concentration of the salt formed by the ampholyte as a base with an anion.

6. $\mathrm{k}_{\mathrm{a}}$ and $\mathrm{kb}$ - the dissociation constants of the ampholyte as acid and base respectively.

7. $k_{1}$ and $k_{11}$-the dissociation constants of the two ampholyte salts; $k_{1}$ of that with the metal-cation and $k_{11}$ of that with the acidanion.

Then we shall define as:

$\frac{\mathrm{a}}{\mathrm{a}}=\rho$-the dissociation residue

$\frac{\mathrm{a}^{*}}{\mathrm{a}}=\alpha^{-}$-the degree of dissociation of the cations 


$$
\begin{aligned}
& \frac{\mathrm{a}^{\prime}}{\mathfrak{a}}=\alpha^{\prime} \text {-the degree of dissociation of the anions } \\
& \frac{\mathrm{SI}}{\mathfrak{a}}=\sigma_{I} \text { the degree of dissociation of the cation salt } \\
& \frac{\mathrm{SII}}{\mathfrak{a}}=\sigma_{\mathrm{II}} \text {-the degree of dissociation of the anion salt }
\end{aligned}
$$

The following equilibrium conditions obtain:

$$
\begin{gathered}
a^{\prime} \cdot h^{\prime}=k_{a} \cdot a \\
a^{\prime} \cdot o h^{\prime}=k_{b} \cdot a \\
a^{\prime} \cdot i^{\prime}=k_{I} \cdot s_{I} \\
a^{\prime} \cdot i^{\prime}=k_{I I} \cdot s_{I I} \\
a+a^{\circ}+a^{\prime}+s_{I}+s_{I}=a \\
h^{\prime} \cdot o h^{\prime}=k_{w} .
\end{gathered}
$$

Hence for $\rho=\frac{a}{a}$

$$
\rho=\frac{1}{1+\frac{\mathrm{k}_{\mathrm{b}}}{\mathrm{k}_{\mathrm{w}}} \cdot \mathrm{h} \cdot\left(1+\frac{\mathrm{i}^{\prime}}{\mathrm{k}_{\mathrm{II}}}\right)+\frac{\mathrm{k}_{\mathrm{a}}}{\mathrm{h}^{\cdot}}\left(1+\frac{\mathrm{i}^{\prime}}{\mathrm{k}_{\mathrm{I}}}\right)}
$$

The $\rho$-curve is formally the same as that without salt-formation, except that its parameters have a slightly different meaning. The path of the curve is such as it would be if the two dissociation constants of the ampholyte were increased by the presence of the electrolyte in excess (just as in the case of the $\rho$-curve of a simple acid or base).

A further analytic study yields for the $\left[\mathrm{H}^{+}\right]$(or $\mathrm{h}$ ) at the maximum value of $\rho$

$$
\left[\mathrm{H}^{+}\right] \text {at } \rho_{\max }=\sqrt{\frac{\mathrm{k}_{\mathrm{o}}}{\mathrm{k}_{\mathrm{b}}} \cdot \mathrm{k}_{\mathrm{w}} \frac{\left(1+\frac{\mathrm{i}^{\circ}}{\mathrm{k}_{\mathrm{I}}}\right)}{\left(1+\frac{\mathrm{i}^{\prime}}{\mathrm{k}_{\mathrm{II}}}\right)}}
$$

This value should be compared with that for $\mathrm{h}$ at $\rho_{\mathrm{max}}$ given in (1) page 65 . And for $\rho_{\max }$ itself we find

$$
\rho_{\max }=\frac{1}{1+2 \sqrt{\frac{\mathrm{k}_{\mathrm{a}} \mathrm{k}_{\mathrm{b}}}{\mathrm{k}_{\mathrm{w}}}\left(1+\frac{\mathrm{i}^{\prime}}{\mathrm{k}_{\mathrm{I}}}\right)\left(1+\frac{\mathrm{i}^{\prime}}{\mathrm{k}_{\mathrm{II}}}\right)}}
$$


The curves in figure 23 below give an idea of the change in the $\rho$-curve in the presence of salts. Here we have a solution of an ampholyte with $\mathrm{k}_{\mathrm{a}}=10^{-7}$ and $\mathrm{k}_{\mathrm{b}}=10^{-11}$. Its $\rho$-curve may be thought of as being the composite of the $\rho$-curves of an acid with $\mathrm{k}_{\mathrm{a}}=10^{-7}$ (marked $\rho_{\mathrm{a}}$ on the curve) and of a base with $\mathrm{k}_{\mathrm{b}}=\frac{\mathrm{k}_{\mathrm{w}}}{10^{-11}}=$ $10^{-3}$ (marked $\rho_{\mathrm{b}}$ on the curve). $\rho_{\max }$ corresponds to $\mathrm{pH} 5$. Let us suppose that changes in $\mathrm{pH}$ are brought about by the addition of buffer mixtures which do not form true salts with the ampholyte. Now let us add to our solution a salt whose anion will combine with the ampholyte to form a salt with the constant $\mathrm{k}_{\mathrm{I}}=0.1$, and whose cation will likewise form a salt with the constant $k_{\text {II }}=0.01$. This will result in the displacement of the $\rho_{\mathrm{a}}$ curve to $\rho_{\mathrm{a}}^{\prime}$ and of the $\rho_{\mathrm{b}}$ curve to $\rho^{\prime}{ }_{b}$. The total new curve can be approximately pieced together from the curves $\rho^{\prime}{ }_{\mathrm{a}}$ and $\rho_{\mathrm{b}}^{\prime}$ and by rounding out the angle

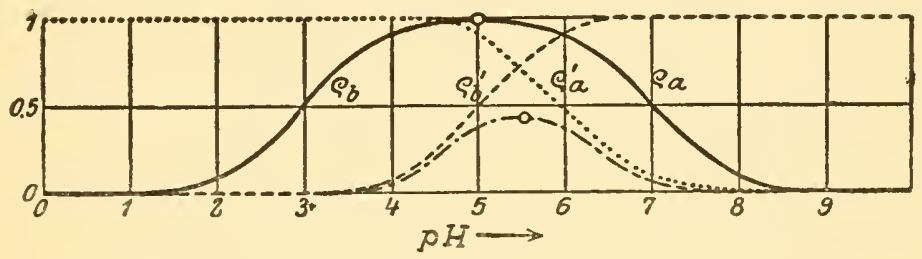

FIG. 23

at which these two meet but leaving the maximum above the same point on the abscissa. In this way the lowest curve of the figure (drawn with the - - - - line) is obtained. The maxima of the original and of this latter curve are indicated by small circles. We see that the maximum has been changed in a twofold way by the addition of the salt. In the first place, its ordinate value became smaller, and this is because the $\rho_{a}^{\prime}$ and $\rho_{b}^{\prime}$-curves are situated closer to each other than the $\rho_{\mathrm{z}^{-}}$and $\rho_{\mathrm{b}}$-curves, secondly, the maximum was displaced horizontally towards the right, and this is because the values of $k_{I}$ and $k_{I I}$ are different. In the case where $k_{I}=k_{I I}$ only the first ordinate displacement would occur. But in this case the $\rho$ maximum is in the first place diminished in its absolute value, and, secondly, it is displaced to a different $\mathrm{pH}$ value.

On superficial consideration it might be supposed that these maxima represent the two isoelectric points, and consequently that the isoelectric points were shifted by the addition of the salt. But this is not the case as we shall at once see. 
41. The influence of salt-formation upon the isoelectric point

Of particular importance in this connection is the definition of the isoelectric point. Earlier in this book it could well be defined as:

$$
\text { I P }=\left[\mathrm{H}^{+}\right] \text {for } \rho \max .=\sqrt{\frac{\mathrm{k}_{\mathrm{a}}}{\mathrm{k}_{\mathrm{b}}} \mathrm{k}_{\mathrm{w}}}
$$

But this definition is only justified in the absence of true salts of the ampholyte. The logical general definition of the isoelectric point is given as that hydrion concentration at which an equal number of positive and negative ampholyte ions are present. In order to transfer this definition analytically to this particular case it is necessary to introduce the conception of the degree of charge, $\lambda$. By means of quantitative transference experiments it can be determined how much of the ampholyte is transported in a current of definite intensity, in a unit of time and in the direction 0 the current, while the amount migrating against the current is calculated as a negative quantity. When the ampholyte is solely in the form of cations, then its transference is maximal.

In general, only a fraction of this maximal amount is transferred. It is this fraction that we shall designate as the degree of charge $\lambda$. It is primarily dependent upon the hydrion concentration and also upon true salt formation by the ampholyte. It must be expressed as

$$
\lambda=\alpha^{\cdot}-\alpha^{\prime}
$$

$\lambda$ may be either positive or negative. The isoelectric point can now be defined as that $\left[\mathrm{H}^{+}\right]$at which $\alpha^{\circ}=\alpha^{\prime}$, and therefore, when $\lambda=0$.

The calculation of $\lambda$ is based upon the following relations (compare with (2) and (1), page 142).

$$
\begin{aligned}
& a^{\circ}=\frac{a \cdot k_{b}}{o h^{\prime}} \\
& a^{\prime}=\frac{a \cdot k_{a}}{h^{\circ}}
\end{aligned}
$$

\section{Hence}

$$
a^{\cdot}-a^{\prime}=\frac{a \cdot k_{b}}{o h^{\prime}}-\frac{a \cdot k_{a}}{h^{*}}
$$


Consequently

$$
\frac{a^{2}-a^{\prime}}{a}=\alpha^{\prime}-\alpha^{\prime}=\lambda=\rho\left(\frac{k_{b}}{o h^{\prime}}-\frac{k_{a}}{h^{\prime}}\right)
$$

By substituting the value of $\rho$ from (7) p. 142 .

$$
\lambda=\frac{\frac{k_{b} \cdot h^{\cdot}}{k_{w}}-\frac{k_{a}}{h^{\circ}}}{1+\frac{k_{b} \cdot h^{\prime}}{k_{w}}\left(1+\frac{i^{\prime}}{k_{I I}}\right)+\frac{k_{\mathrm{a}}}{h}\left(1+\frac{i^{\prime}}{k_{I}}\right)}
$$

In the special case, when no true salt-formation occurs, i.e., when either no extraneous ions are present, or when their affinity for the ampholyte ions is infinitely small (when $\mathrm{i}^{*}=\mathrm{i}^{1}=0$, or $\mathrm{k}_{\mathrm{I}}=\mathrm{k}_{\mathrm{II}}=\infty$ ) expression (10) becomes

$$
\lambda_{0}=\frac{\frac{k_{b}}{0 h^{\prime}}-\frac{k_{a}}{h}}{1+\frac{k_{b}}{0 h^{\prime}}+\frac{k_{a}}{h_{1}}}
$$

The isoelectric point is, therefore, that $\left[\mathrm{H}^{+}\right]$at which $\lambda=0$. Hence, from the general expression for $\lambda$ as well as for the special expression for $\lambda_{0}$ we derive the same value for the isoelectric point as we did originally.

$$
\text { I.P. }=\sqrt{\frac{k_{a}}{k_{b}} k_{w}}
$$

This means that the isoelectric point is not deflected by true salt-formation, which is a very remarkable conclusion. And since it has just been demonstrated that the value of $\rho_{\max }$ is deflected, it follows that in the case of true salt-formation the values of $\rho_{\max }$ and $I . P$. are not identical.

\section{The extension of the conception of the reduced dissociation constant}

The buffer equation in its last interpretation stated that in the presence of an excess of neutral salt ((4), page 129).

$$
\left[\mathrm{H}^{+}\right]=\mathrm{k}^{\prime} \frac{[\text { free acid] }}{\text { [salt of the acid] }}
$$


The difference between $\mathrm{k}^{\prime}$ and $\mathrm{k}$ was interpreted in the sense of the change of activity of the acid-ions because of the presence of the neutral salt. If we should furthermore assume that the cation of the neutral salt can form a true salt with the anion of the weak acid as we have done formerly, and, as we still must assume in certain cases-then we find the following:

Let us consider only the dissociation residue, $\rho$. The first approximation for $\rho$ was

$$
\rho=\frac{1}{1+\frac{k}{h}}
$$

The activity theory demands that, in the presence of neutral salt, we substitute for the constant $k$ the constant $k^{\prime}$ reduced to the corresponding salt concentration, and hence,

$$
\rho=\frac{1}{1+\frac{k^{\prime}}{h}}
$$

If we now assume that the anion of the buffer acid and the neutral salt cation (of concentration i) form a salt whose dissociation constant is constant $k_{8}$, then according to (I), page 140 ,

$$
\rho=\frac{1}{1+\frac{k^{\prime}}{h}\left(\frac{i}{k_{g}}+1\right)}
$$

Equation (3) differs from (2) inasmuch as the value of $\mathrm{k}^{\prime}$ in (2) is substituted in (3) by $\mathrm{k}^{\prime}\left(\frac{\mathrm{i}}{\mathrm{k}_{\mathrm{s}}}+1\right)$. Since with a constant salt. content the value within the parenthesis is a constant, this entire difference can be summarized as $\mathrm{k}^{\prime \prime}$, and equation (3) becomes

$$
\rho=\frac{1}{1+\frac{k^{\prime \prime}}{h}}
$$

In the formal sense, nothing is changed, except that another value of $\mathrm{k}$ is used, i.e., the $\rho$-curve is only horizontally displaced; this is a formal procedure similar to that when $\mathrm{k}^{\prime}$ was earlier substituted for $\mathrm{k}$. 
It could be doubted if the conception of "a constant reduced to a definite salt content" would still hold, if the premise of the total dissociation of all salts were invalidated. It has been shown that such doubt is unjustifiable. Only it cannot always be distinguished in individual cases whether the apparent change of $\mathrm{k}$ to $\mathrm{k}^{\prime}$ is based upon an activity effect or upon true salt-formation. As far as the physiological utilization of the "reduced constant" is concerned this is of no import; for our ionic equilibria can still be calculated if only the "reduced dissociation constants" of the acid for the corresponding neutral salt content of the solution is known, no matter how complicated the theoretical derivation of this constant may be. In any event, by using the properly reduced constant, the amount of undissociated acid molecules can be irrefutably calculated. What remains undetermined are the relative amount of the remaining acid molecules dissociated into ions and those bound into true salt molecules.

All of the above considerations are valid for the univalent ions, but if a weak acid forms a salt with a divalent ion (as, for example, acetate buffer $+\mathrm{CaCl}_{2}$ ), then not only does the horizontal displacement of the $\rho$-curve ensue, but the curve simultaneously also becomes steeper. Concerning the more detailed calculation of these relations the original article ${ }^{3}$ should be referred to. Special complications also arise when we assume the acid molecules in solution are associated with two or more molecular species and approach the colloidal state. Concerning this aspect also the above mentioned original article should be consulted.

${ }^{3}$ L. Michaelis, Biochem. Zeitschr. 106, 83 (1920). 


\section{CHAPTER V}

\section{Electrolytic Dissociation in Non-aqueous Solutions}

\section{SUMMARY OF CONTENTS}

The little that is known concerning dissociation in non-aqueous solutions is described, insofar as it promises to become of physiological interest.

43. Apart from a few exceptions, such as, for example, hydrocyanic acid, the electrolytic dissociation is not nearly as great in any solvent as it is in water. The smaller the dielectric constant of a solvent the smaller is its dissociating capacity for the electrolytes dissolved in it. In the living organism there are in addition to the watery phases also lipoidal phases. These are chemical systems in which the fundamental substance is not water but substances of the nature of true fats, lecithins, and related compounds. These phases form in the organism either microscopic or else ultramicroscopic structures as colloidal solutions, or, continuous phases, such as in the medullary sheaths of the nerves. Many membranes, particularly, contain lipoids. Whether the lipoids form a continuous phase, or whether the lipoidal and the non-lipoidal constituents are arranged in mosaic form within the membrane structure, is not definitely established. But whether the lipoid substances are continuous, or whether they are to be conceived of as forming a "disperse" phase within an aqueous phase serving as a dispersion medium, in any event, every individual lipoid droplet is a chemical system with a lower dielectric constant than water, and which, as a solvent for electrolytes, behaves differently from water.

Our knowledge concerning the dissociation of electrolytes in such organic solvents is still very full of gaps. It is a pressing need to fill these gaps, for that alone will enable us to explain the rôle of the lipoids, especially as a cause of bioelectric phenomena. The reason for our inadequate information lies in the difficulties inherent to the methods. The method which, in aqueous solutions, is the most useful in the study of dissociation, the measurement of conductivity, 
is very much more difficult when applied to lipoids. This is because the conductivity of the lipoids themselves is so extraordinarily small, and electrolytes dissolved in them are so poorly dissociated that the total conductivity in all such cases remains very small. Besides, it is generally quite impossible to obtain the value of $\Lambda_{\infty}$ by extrapolation. The other method which led to further results was that of the concentration chains. The measurement of the electromotive force in these chains is easily carried out in aqueous solutions. These methods are all based upon the measurements of electric currents, or more specially, upon compensation of currents by oppositely directed currents. The lipoids offer such tremendous resistance to the electric current that measurable current intensity is not generally obtainable. It is chiefly electrostatic tension that can be measured in these cases. Special instruments have been devized for this purpose, such as the quadrant electrometer by Thomsen and its newer modification, the binant electrometer, by Dolezalek. These methods were successfully applied by Cremer, ${ }^{1}$ Haber and Klemensiewicz, ${ }^{2}$ Loeb, ${ }^{3}$ and Beutner ${ }^{4}$ to determine the degree of dissociation of electrolytes, covering, to be sure, only a part of the above developed general purpose. Only a few points of support are available for attacking our problem. First, a certain regularity discovered by $\mathrm{P}$. Walden ${ }^{5}$ must be mentioned. As was stated earlier (page 14), Walden found that on comparing those concentrations (c) of a given electrolyte which have the same degree of dissociation in different solvents, it appeared that the cubic roots of the concentrations were directly proportional to the dielectric constants D of the correspond$i^{\text {ng solvents, or, }}$

$$
\mathrm{D}_{1}: \mathrm{D}_{2}=\sqrt[3]{\mathrm{c}_{1}}: \sqrt[3]{\mathrm{c}_{2}}
$$

Let us find, for instance, that concentration $\mathrm{c}$ of an electrolyte in different solvents at which the degree of dissociation, $\alpha=0.5$. Now

1 Cremer, Zeitschr. f. Biol. 47, 1 (1906).

${ }^{2}$ Haber and Klemensiewicz, Ann. d. Physik [4] 26, 927 (1908).

3 Jacques Loeb, Journ. of Gen. Physiol. 3, 667 (1921).

${ }^{4}$ R. Beutner, Die Entstehung elektrischer Ströme in lebenden Geweben. Stuttgart 1920.

${ }^{5}$ P. Walden, Zeitschr. f. physik. Chem. 54, 228 (1905) ; 94, 263 and 372 (1920). 
according to Ostwald's dilution law, or, according to the mass law,

$$
\frac{\alpha^{2}}{1-\alpha} \mathrm{c}=\mathrm{k}
$$

where $\mathrm{k}$ is the dissociation constant of the electrolyte. By letting $\alpha=0.5$ we obtain

$$
\frac{0.25}{0.5} \mathrm{c}=\mathrm{k}
$$

or

$$
c=2 k
$$

therefore, $\mathrm{c}$ is proportional to $\mathrm{k}$.

By substituting $\mathrm{k}$ for $\mathrm{c}$ in the above equation

$$
D_{1}: D_{2}=\sqrt[3]{k_{1}}: \sqrt[3]{k_{2}}
$$

where $k_{1}$ and $k_{2}$ are the dissociation constants of the electrolytes in the two solvents. Then Walden also found that actually this relation was fairly valid for a large series of electrolytes. But the electrolytes tested by him were exclusively of the strong type (such as $\mathrm{KCl}, \mathrm{NaI}, \mathrm{AgNO}_{3}, \mathrm{~N}\left(\mathrm{C}_{2} \mathrm{H}_{5}\right)_{4} \mathrm{I}$, etc.) to which Ostwald's dilution law cannot be applied. Thus there are still difficulties in the way of establishing Walden's theory.

Certain further elucidation concerning the dissociation of electrolytes in organic solvents is found in the investigations of R. Beutner (1. c.):

Many of the inorganic electrolytes, such as $\mathrm{KCl}$, very soluble in water, are not at all measurably soluble in oils. ${ }^{6} \mathrm{~A}$ much better insight can be had by working with organic acids and bases and with their salts. Thus, if salicylic acid is dissolved in nitrobenzene, the conductivity increases perceptibly; likewise with dimethyltoluidine. Now, if equivalent amounts of salicylic acid and dimethyltoluidine be dissolved in the same solvent, the resulting conductivity is appreciably greater than the sum of the individual conductivities. We may conclude from this that the salt is more strongly dissociated than the acid or the base, as is always the case in aqueous solutions.

"The author uses the term "oil," in this connection and in subsequent chapters, to designate any oily liquid, generally forming a separate phase when in contact with an aqueous phase.-Translator. 
But also the hydrolysis of this salt is much greater in oil than it is in water. The greater part of the acid and of the base persist alongside of each other. This can be seen from the following:

When we add more base to a mixture of much acid and little base, then we should expect that the conductivity would rise in proportion to the added base, if the latter was completely utilized for salt formation. But it is observed that the conductivity increases less than expected. Consequently, it must be assumed that the base was not completely used up for the formation of salt, i.e., that the salt remains extensively hydrolyzed, even in the presence of an excess of acid.

As experimental proof for this view the following observations made by Beutner may be quoted:

$1 . /$ solution of salicylic acid in nitrobenzene showed at a high temperature (not so soluble in the cold) a conductivity of 3.5 reciprocal megohms; calculated for room temperature..................

$1 M$ solution of dimethyltoluidine in nitrobenzene.........................

$1 M$ solution of salicylic acid $+1 M$ dimethyltoluidine in nitrobenzene........... 430 recipr. megohms

$1 / 25 M$ solution of salicylic acid $+1 / 100 M$ dimethyltoluidine in nitrobenzene...... 18.5 recipr. megohms $1 / 25 M$ salicylic acid $+1 / 50 M$ dimethyltoluidine in nitrobenzene............ 23.8 recipr. megohms (instead of 37 , as it should have been, if all the base added were used for salt-formation)

$1 / 25 M$ salicylic acid $+1 / 25 M$ dimethyltoluidine in nitrobenzene................

2 recipr. megohms

0.1 recipr. megohms (1) 27.5 recipr. megohms (instead of 74)

A quantitative utilization of these figures in the calculation of dissociation constants can not unfortunately be carried out, for Ostwald's dilution law, i.e., the mass law, is not valid for electrolyte solutions in oils on grounds which are as yet to be worked out. This is probably due to molecular associations or aggregates, which electroneutral molecules tend so strongly to form.

A second difference is in the fact that in oily solutions there are no acids or bases which are as strongly dissociated as the salts: there are no "strong" acids and bases. Here we have only weak electrolytes-namely all salts, and extremely weak electrolytesnamely, all acids and bases. 
The definition of acid and alkaline reaction would be the same as in aqueous solution; at the neutral reaction $\left[\mathrm{H}^{+}\right]=\left[\mathrm{OH}^{-}\right]$, but methods for the determination of the reaction are still lacking. A gas chain with an oily liquid has not yet been investigated. Nor is the dissociation constant of water (in oil) known

$$
\frac{\left[\mathrm{H}^{+}\right]\left[\mathrm{OH}^{-}\right]}{\mathrm{H}_{2} \mathrm{O}}=\mathrm{k}_{\mathrm{w}}
$$

(where $\left[\mathrm{H}_{2} \mathrm{O}\right]$ represents again a value proportional to the vapor pressure). Doubtlessly it depends to a great extent upon the nature of the oil and especially upon its dielectric constant. In our present state of knowledge it is useless to attempt to apply any indicator method to oil phases. All that can be done at present is to disclose this great gap.

The principal difference between the two classes of electrolytes, 1-acids and bases, 2-salts, appears to be still evident even in solvents with small dielectric constants. In this connection also $\mathrm{H}$ - and $\mathrm{OH}$-ions exhibit their specific peculiarity. This is all the more remarkable, since the other peculiarity of the $\mathrm{H}$ - and $\mathrm{OH}$-ions, i. e., their abnormally high conductivity, is not in evidence in these solvents. $\mathrm{H}$ - and $\mathrm{OH}-\mathrm{ions}$, for example, have no greater motility in organic water-free solvents than alkali or halogen-ions. The abnormally high conductivity of $\mathrm{H}$ - and $\mathrm{OH}$-ions was explained by Arrhenius in the following way: When a moving $\mathrm{H}$-ion comes in contact with a $\mathrm{H}_{2} \mathrm{O}$-molecule, it combines with the $\mathrm{OH}$-group of the latter which immediately liberates its $\mathrm{H}$-group as an $\mathrm{H}^{+}$-ion. In this way in the process of conduction the portion of the path corresponding to the thickness of the $\mathrm{H}_{2} \mathrm{O}$-molecule is saved, and an apparent impression of an abnormally high motility of the H-ion results. The same holds for the $\mathrm{OH}-\mathrm{ion}$. In general it appears that in every solvent those ions which the solvent itself is capable of yielding possess an abnormally high conductivity. This theory, is similar to the one once proposed by Grotthus concerning general electrolytic conduction, and which had been dominant before the Arrhenius theory of dissociation appeared. 
PART II

The Ions, Particularly the Hydrogen Ions, as Sources of Electric Potential Differences 



\section{INTRODUCTION}

44. All solutions of electrolytes contain equal amounts of positively and negatively charged ions. Deviations from this proposition are possible only to so slight an extent that it is beyond the scope of analytical chemical methods to detect the inequalities between the two ionic species. But, nevertheless, because of the large amounts of free electricity which arise in such cases, this inequality is demonstrable by physical methods, and it is the cause of a number of electric phenomena. It is not our purpose to treat of these as extensively as of ionic equilibria. But since the methods for measuring hydrion concentration are based upon these phenomena they must be discussed here insofar as our understanding of these methods requires it. Furthermore, our explanations in general will be made in the direction in which they are to have a probable physiological bearing.

Among the potential differences brought about by the unequal partition of ions, several groups may be differentiated; such as: 1. electrode potentials, or those which are located on the surface of a metal in contact with a solution; 2 . diffusion potentials, or those which ensue at the contact of two different electrolyte solutions or of two solutions of unequal concentration; 3. phase boundary potentials which are generally located on the boundary surface between two phases, in such a manner that one phase shows a potential towards the other phase; 4 . membrane potentials, which are based upon the impermeability of certain membranes for certain ions; 5. adsorption potentials which also develop on a phase boundary surface, but in such a way that the boundary layer of a phase exhibits a potential difference towards the rest of the same phase. 


\section{CHAPTER VI}

\section{The Electrode Potentials \\ SUMMARY OF CONTENTS}

Nernst's theory of metal electrode potentials is developed and applied to concentration chains, of which the hydrogen gas chains are the most important for physiological work. The application of Nernst's theory to chains with various metal electrodes is briefly explained and the electromotive series is described.

\section{The single potential of an electrode}

A metal immersed in a solution generally shows a difference of potential towards the solution. This long familiar fact has been theoretically worked out after the establishment of the ionic theory and developed by Nernst. It has long been recognized that this potential difference between a metal and a solution depends, first, upon the nature of the metal, secondly, upon the composition of the solution, and thirdly, to a small extent, upon the temperature. The composition of the solution refers to the solvent as well as to the solute electrolyte. As the solvent, water alone will first be considered. As far as the electrolyte is concerned, only the concentration of that ionic species is of importance which is capable of yielding ions of the metal involved. All other ionic species which may be present in the solution are immaterial as far as the value of the potential difference is concerned. Thus the potential of a silver electrode towards a $0.001 \mathrm{~N} \mathrm{AgNO}_{3}$ solution is the same as that towards a $0.001 \mathrm{~N}$ solution of $\mathrm{AgClO}_{3}$, or of any silver salt, i.e., if a galvanic chain were to be made of these two electrodes then the resulting E.M.F. $=0$. But if one silver electrode is immersed in a $0.01 \mathrm{~N} \mathrm{AgNO}_{3}$ solution on one side and another silver electrode is immersed in a $0.001 \mathrm{~N} \mathrm{AgNO}_{3}$ solution on the other side, a current will be generated by this chain. Whether or not there be present in these solutions another salt, $0.01 \mathrm{~N} \mathrm{KNO}_{3}$ for example, is immaterial, insofar as this salt does not by chemical reaction alter the con- 
centration of the dissolved $\mathrm{Ag}^{+}$-ions. On the other hand, the addition of $\mathrm{NaCl}$ would precipitate almost all of the $\mathrm{Ag}^{+}$as $\mathrm{AgCl}$, and only insofar as the precipitation is concerned has $\mathrm{NaCl}$ an effect upon the potential.

Therefore, at the very beginning of our theoretical derivation of Nernst's electrode potential we shall state the experimental fact that the potential difference of a metal toward a solution depends only upon the concentration of the ions of the same metal present in the solution. This can be formulated as:

$$
\pi=\mathrm{f}(\mathrm{c})
$$

where $\pi$ is the potential and $\mathrm{c}$ is the concentration of the ionic species concerned. Now it is our purpose to define the function $f(c)$ in greater detail. To this end let us utilize the principle of thermodynamics:

When a chemical system passes reversibly from one state into another, the maximal work which can be gained from such change of state is independent of the manner in which this change occurs as long as it remains reversible.

Now then, when a metal electrode is used as one pole of a galvanic element, experience shows that the process occurring at this electrode, on closing the circuit, is in many cases reversible. According to the conditions and according to the direction of the resulting current, the metal will be either dissolved or deposited. Thus, if the current is allowed to flow until one gram ion of $\mathrm{Ag}^{+}$is dissolved, and then by means of another source of current an oppositely directed current of the same ampere-hour value as the first is sent through the same system, then exactly the same amount of silver will be deposited as had been previously dissolved. Hence the process is reversible. The initial and final states of this system (if the process is not reversed) differ, inasmuch as at the end there is one gram-ion of $\mathrm{Ag}^{+}$ more in the solution than in the beginning. This change in the system can be brought about in another reversible way, different from the electrolytic process, i.e., by removing water from the solution until it is correspondingly concentrated and then restoring the volume by the addition of a solution of the same higher concentration. The removal of the water must needs be performed in a reversible manner, such as by means of an osmotic piston impermeable to Ag-ions. With such a piston the solution is compressed, as it were, until its concentration reaches the desired value. 
In the one case we can calculate the electrical work and in the other case the osmotic work performed. The electrical work is equal to the product of the potential difference $\pi$ and the transported amount of electricity, or 96,450 coulombs, which is attached to one gramion of silver. This amount of 96,450 coulombs is designated as $1 \mathrm{~F}$ (faraday). The electric work $\mathrm{W}$ is, therefore, $\pi \mathrm{F}$. If the potential were increased to $\pi+\mathrm{d} \pi$, then the work would be increased by the amount of $\mathrm{d} W=\mathrm{F} \mathrm{d} \pi$.

The osmotic work performed in the above cited case consists entirely of the work of compression; the restoration to the original volume does not demand nor yield any work, for no change of concentration is involved. This osmotic pressure depends upon the osmotic pressure against which it is exerted, which means that it depends upon the concentration of the Ag-ions already present in the solution. Therefore it can not be defined without going into further explanation. But this much can be said: if the osmotic process consists of a dilution, and if the osmotic pressure is at first $p$ and then $\mathrm{p}-\mathrm{d} \pi$, then the work to be obtained in the latter case is less by $\mathrm{v} \cdot \mathrm{dp}$ than in the first case. Here $\mathrm{v}$ stands for the volume containing one gram-ion in solution. When, on the other hand, the osmotic process consists of compression (concentration), then the sign is to be reversed and taken as negative.

Thus the osmotic work must be equal to the electrical work, which can be expressed in terms of differentials as

$$
\mathrm{F} \mathrm{d} \pi=-\mathrm{v} \mathrm{dp}
$$

Since $p v=R T$ and $v=\frac{R T}{p}$, hence

$$
\begin{aligned}
\mathrm{Fd} \pi & =-\frac{\mathrm{RT}}{\mathrm{p}} \mathrm{d} \mathrm{p} \\
\mathrm{d} \pi & =-\frac{\mathrm{RT}}{\mathrm{F}} \cdot \frac{\mathrm{d} \mathrm{p}}{\mathrm{p}}
\end{aligned}
$$

and integrating:

$$
\pi=\frac{\mathrm{RT}}{\mathrm{F}} \ln \frac{1}{\mathrm{p}}+\text { Constant }
$$


This integration constant can be replaced by $\frac{\mathrm{RT}}{\mathrm{F}} \times \ln \mathrm{P}$ and our equation becomes

$$
\pi=\frac{\mathrm{RT}}{\mathrm{F}} \times \ln \frac{\mathrm{P}}{\mathrm{p}}
$$

When $\mathrm{p}=\mathrm{P}$, then $\pi=0$. Hence $\mathrm{P}$ represents that osmotic pressure of the ionic species involved against which the electrode has 0 potential. Herein lies the physical interpretation or signifcance of the integration constant.

Since in very dilute solutions the osmotic pressure is proportional to the concentration, the above equation can also be stated as

$$
\pi=\frac{\mathrm{RT}}{\mathrm{F}} \times \ln \frac{\mathrm{C}}{\mathrm{c}}
$$

where $\mathrm{c}$ is the ionic concentration in the given solution and $\mathrm{C}$ that concentration against which the electrode potential would be $=0$.

The meaning of the osmotic pressure of an ionic species must be here further dealt with in somewhat greater detail. For extremely dilute solutions there is no difficulty in this respect, for here concentration and pressure are proportional to each other. But in higher concentrations this is no longer strictly true, and in such cases equation (1) above must be used, in terms of pressures. Formerly no doubt was entertained in accepting as the osmotic pressure that value which was obtained from freezing point determinations. Thus, for example, if it was found that the lowering of the freezing point of a $1 \mathrm{~N} \mathrm{KCl}$ solution was 1.8 times as great as that of a $1 \mathrm{~N}$ cane-sugar solution, it was concluded that the "osmotic pressure" of the $\mathrm{KCl}$ solution was 1.8 times as great. Furthermore, it was assumed that, because of incomplete dissociation, there were 1.8 times (instead of twice) as many individual molecules (i.e., ions) in the $\mathrm{KCl}$ solution as in the sugar solution. Now we assume that there are actually twice as many molecules in the $\mathrm{KCl}$ solution (see page 116), but that the electrostatic forces have an effect upon the lowering of the freezing point. We have also shown that these forces also exercise another influence upon the active mass of the ions, and it is this active mass that we must obviously take to represent the "pressures" $\mathrm{p}$ and $\mathrm{P}$ above. Thus, when in following the old convention, we speak of "osmotic pressure," it must be 
realized that we must no longer take it in the sense of that "osmotic pressure" which had been derived from freezing point determinations, but that it would be much better to substitute the term "active mass" for that of "osmotic pressure."

The active mass is obtained from the concentration by multiplying the latter by the factor $f_{a}$, the factor being so chosen that the otherwise approximate equation (2) should correspond exactly to the measured E.M.F. Properly speaking, this brings us into a vicious circle. For, an equation such as (2) is set up, it is observed that it is not quite correct, some of its values are altered by means of empiric factors, and then, naturally, it is found that the formula is entirely correct. While there is a grain of truth in this objection, it is not entirely justifiable, for we have shown above, in a quantitative way, the causes for the necessity of these corrections, and, in a few suitable cases, it was also quantitatively possible to arrive at fairly accurate results on the basis of certain verified assumptions. Furthermore, we have seen that even the uncorrected equation is evidently entirely valid, as a limiting law, for high dilutions. It is beyond doubt that in the near future the activity factor will be derived from other than electromotive force data. Then the reason for the above vicious circle objection will disappear. Considering the newness of the conception of the activity factor, it appeared only proper to point out these difficulties and to forestall this all too easily raised objection.

If instead of the activity theory we choose the theory given on page 131, we could then state even more clearly, the following: The concentration $\mathrm{C}$ of the current yielding ions against which the metal electrode has a potential of 0 , varies with the amount and the nature of all the ions present in solution (not only the current producing ions). Therefore, when the concentration of the currentyielding ions is greatly altered, without maintaining the total electrolyte content of the solution at a constant level by a corresponding change of the non-current-yielding ions, then not only is the value of c changed, but also that of $\mathrm{C}$. Hence, in the equation $\pi=\frac{\mathrm{RT}}{\mathrm{F}} \ln \frac{\mathrm{C}}{\mathrm{c}}$ the value $\mathrm{C}$ cannot be strictly considered as being a constant.

\section{The concentration chain}

There is no adequate method for the measurement of any given single potential of an electrode, but two electrodes are put together 
into a chain, its E.M.F. is measured and taken to represent the difference between the single potentials. Chains may be made up of two different metals, or of electrodes of the same metal in contact with solutions of different concentrations, these latter being the concentration-chains. In accord with the main purpose of this book, we shall concern ourselves chiefly with the theory of such concentration chains.

Let us take two silver electrodes immersed in solutions of $\mathrm{AgNO}_{3}$ whose concentration on the left side is $c_{1}$ and on the right side $c_{2}$. We shall assume that at the place of contact of the two solutions there exists no difference of potentials, a condition frequently realized and with which we shall later deal separately. We have then in this case

$$
\pi_{1}=\frac{\mathrm{RT}}{\mathrm{F}} \ln \frac{\mathrm{C}}{\mathrm{c}_{1}} \quad \pi_{2}=\frac{\mathrm{RT}}{\mathrm{F}} \ln \frac{\mathrm{C}}{\mathrm{c}_{2}}
$$

and the EMF

$$
\begin{aligned}
\mathrm{E}=\pi_{1}-\pi_{2} & =\frac{\mathrm{RT}}{\mathrm{F}} \ln \frac{\mathrm{C}}{\mathrm{c}_{1}} \cdot \frac{\mathrm{c}_{2}}{\mathrm{C}} \\
\mathrm{E} & =\frac{\mathrm{RT}}{\mathrm{F}} \ln \frac{\mathrm{c}_{2}}{\mathrm{c}_{1}}
\end{aligned}
$$

Hence the constant $\mathrm{C}$ disappears from the equation. Such chains are easily realized, and, therefore, we must first discuss the numerical evaluation of the factor $R$. Since it is desired to obtain $E$ in volts, it is necessary to calculate the gas constant $R$ in terms of electrostatic units.

Note: When the valence of the current-yielding ions is $n$, then the equation correspondingly becomes

$$
\mathrm{E}=\frac{\mathrm{RT}}{\mathrm{nF}} \ln \frac{\mathrm{c}_{2}}{\mathrm{c}_{1}}
$$

47. The numerical evaluation of Nernst's formula in volts

The value $R$ is defined according to the gas laws as

$$
\mathrm{R}=\frac{\mathrm{p} \mathrm{v}}{\mathrm{T}}=\frac{\mathrm{p}_{0} \mathrm{v}_{0}}{273.09}
$$


where $\mathrm{p}$ is the pressure of an ideal gas, $\mathrm{v}$ is the volume which contains one mol of this gas at this pressure and at the absolute temperature $\mathrm{T} ; \mathrm{p}_{\circ}$ and $\mathrm{v}_{\mathrm{o}}$ are the corresponding values for $0^{\circ} \mathrm{C}$., and 273.09 is the absolute temperature value of $0^{\circ} \mathrm{C}$. Taking Berthelot's figure, at $0^{\circ} \mathrm{C}$. at 1 atmosphere of pressure, $v_{\circ}=22,412$ cc.; $p_{\circ}=1$ atmosphere $=76 \mathrm{~cm}$. mercury, which, at $0^{\circ} \mathrm{C}=980,665 \times 76 \times$ $13,595=1,013,280$ dynes per sq. cm. Therefore $\mathrm{R}=83,157,720$ ergs.

$10^{7}$ ergs $=1$ absolute joule; 1 absolute joule $=0.99,966$ international joules. Hence $\mathrm{R}=8.313$ international joules or volt coulombs. 1 faraday, $\mathrm{F}=96,450$ coulombs. Now by substituting these numerical values in our equation we obtain for the E.M.F. of a concentration chain:

$$
\mathrm{E}=\frac{8.313 \cdot(273+\mathrm{t})}{96540} \ln \frac{\mathbf{c}_{1}}{\mathbf{c}_{2}} \text { volts }
$$

Dividing the natural logarithm by 0.4343 we change to the Briggsian logarithms (base 10) and obtain

$$
\begin{aligned}
E & =0.000,1985 \cdot(273+\mathrm{t}) \log \frac{\mathbf{c}_{1}}{\mathbf{c}_{2}} \\
& =\theta \cdot \log \frac{\mathbf{c}_{1}}{\mathbf{c}_{2}}
\end{aligned}
$$

TABLE 22

\begin{tabular}{r|c||c|c||c|c||c|c||c|c||c|c}
\hline $\mathrm{t}$ & $\theta$ & $\mathrm{t}$ & $\theta$ & $\mathrm{t}$ & $\theta$ & $\mathrm{t}$ & $\theta$ & $\mathrm{t}$ & $\theta$ & $\mathrm{t}$ & $\theta$ \\
\hline${ }^{\circ} \mathrm{C}$. & & ${ }^{\circ} \mathrm{C}$. & & ${ }^{\circ} \mathrm{C}$. & & & ${ }^{\circ} \mathrm{C}$. & & & ${ }^{\circ} \mathrm{C}$. & \\
0 & 0.0542 & 16 & 0.0573 & 22 & 0.0585 & 28 & 0.0597 & 34 & 0.0609 & ${ }^{\circ} \mathrm{C}$. & \\
5 & 0.0552 & 17 & 0.0575 & 23 & 0.0587 & 29 & 0.0599 & 35 & 0.0611 & 45 & 0.0621 \\
10 & 0.0561 & 18 & 0.0577 & 24 & 0.0589 & 30 & 0.0601 & 36 & 0.0613 & 50 & 0.0641 \\
12 & 0.0566 & 19 & 0.0579 & 25 & 0.0591 & 31 & 0.0603 & 37 & 0.0615 & 60 & 0.0661 \\
14 & 0.0569 & 20 & 0.0581 & 26 & 0.0593 & 32 & 0.0605 & 38 & 0.0617 & 70 & 0.0680 \\
15 & 0.0571 & 21 & 0.0583 & 27 & 0.0595 & 33 & 0.0607 & 39 & 0.0619 & 80 & 0.0700 \\
\hline
\end{tabular}

Table 22 gives the values of $\theta$ (RT) for various temperatures. By applying these values and the proper experimental conditions, Nernst's equation for concentration chains can be verified with great accuracy. The experimental data of former years frequently did not exceed in accuracy the difference of 2 to 3 millivolts between the 
observed and calculated values. This was because of the imperfect understanding of the dissociation of strong electrolytes and because of the incomplete abolition of the diffusion potential at the contact of the two solutions. It seems that the most appropriate arrangement for the demonstration of the absolute exactness of Nernst's equation for concentration chains is the following:

$$
\begin{array}{c||c|c||c|c}
\mathrm{PCl}\left(\mathrm{c}_{1}\right) & \text { saturated } & \mathrm{HCl}\left(\mathrm{c}_{2}\right) & \mathrm{Pt} \\
\mathrm{H}_{2} & +1.0 \mathrm{~N} \mathrm{KCl} & \mathrm{KCl} \text { solution } & +1.0 \mathrm{~N} \mathrm{KCl} & \mathrm{H}_{2}
\end{array}
$$

Here the concentrations $c_{1}$ and $c_{2}$ of the $\mathrm{HCl}$ must be relatively small in respect to the $\mathrm{KCl}$ concentration $(1.0 \mathrm{~N})$, i.e., they should be between 0.0001 and $0.05 \mathrm{~N}$. The excess of $\mathrm{KCl}$ accomplishes the following purposes: (1) The degree of dissociation of the $\mathrm{HCl}$ (or better, the activity factor of the $\mathrm{H}^{+}$-concentration) is sufficiently near equality in both solutions so that the ratio of the $\mathrm{HCl}$ concentrations is actually equal to that of their $\mathrm{H}^{+}$-activities, and (2) the diffusion potential remains infinitely small. Such chains give E.M.F. values which rarely show a maximum deviation from the calculated figures of 0.0005 volt, and the average of repeated measurements usually agrees perfectly with the theory. Thus this verification of the theory constitutes the convenient method of checking the correctness of the experimental technic for any worker in this field.

\section{Reversible electrodes for concentration chains}

The condition sine qua non, for Nernst's equation is the reversibility of the electrodes. This means that, if the circuit of the chain is closed for a definite period, and then if the exact amount of current produced is sent through the chain in the reversed direction, the original state of the chain must be reëstablished exactly. Each of the two electrodes must be capable of functioning reversibly. This is always the case when a metal is immersed into a solution of its salt and (the circuit being open) no chemical reaction can occur. Thus $\mathrm{Zn}$ in dilute $\mathrm{H}_{2} \mathrm{SO}_{4}$ is not a reversible electrode, since it goes into solution spontaneously, but $\mathrm{Zn}$ in $\mathrm{ZnSO}_{4}$ is such an electrode. $\mathrm{Zn}$ in $\mathrm{CuSO}_{4}$ is not reversible, for the spontaneous reaction occurs, $\mathrm{Zn}+\mathrm{CuSO}_{4} \rightarrow \mathrm{ZnSO}_{4}+\mathrm{Cu}$. $\mathrm{Hg}$ in water is not a reversible elec- 
trode, since, depending upon the amounts of impurities in the water, especially of $\mathrm{O}_{2}$ and $\mathrm{CO}_{2}$, varying traces of $\mathrm{Hg}$ will go into solution. On the other hand, $\mathrm{Hg}$ in a saturated $\mathrm{HgCl}$ solution is a reversible electrode, in spite of the fact that the solubility of calomel depends on the total $\mathrm{Cl}^{-}$content of the solution. For its dissociation occurs in the following manner:

hence

$$
\mathrm{Hg} \mathrm{Cl} \rightleftharpoons \mathrm{Hg}^{+}+\mathrm{Cl}^{-}
$$

$$
\frac{[\mathrm{Hg} \mathrm{Cl}]}{\left[\mathrm{Hg}^{+}\right]\left[\mathrm{Cl}^{-}\right]}=\mathrm{k}
$$

In a saturated calomel solution the concentration of $\mathrm{HgCl}$ molecules is constant, hence,

$$
\left[\mathrm{Hg}^{+}\right] \times\left[\mathrm{Cl}^{-}\right]=\text {a constant }
$$

Therefore the concentration of the $\mathrm{Hg}$-ions in solution is inversely proportional to that of the Cl-ions. Therefore, two Hg-electrodes immersed in two solutions of different concentrations of $\mathrm{KCl}$, both saturated with calomel, form a reversible concentration chain for $\mathrm{Hg}$-ions. Since the ratio of the $\mathrm{Hg}$-ions is reciprocally equal to that of the Cl-ions, such a chain may also be used as a reversible chain for Cl-ions. These are designated as electrodes of the sccond order, or, reversible electrodes for anions. These electrodes may therefore be utilized for the determination of Cl-ion concentrations.

An alloy of a noble with a common metal behaves, in the electromotive sense, as if it consisted of the common metal only. The same is true of gas-electrodes. Platinum (as well as gold, palladium, iridium) absorbs $\mathrm{H}_{2}$-gas from an atmosphere of hydrogen, the absorption equilibrium being determined by the partial pressure of the hydrogen. Such an electrode behaves as if it consisted only of a metal-like, conductive hydrogen, hence it is a reversible electrode for $\mathrm{H}^{+}$-ions.

\section{The theory of gas chains}

We saw above that when a concentration chain is made up of $\mathrm{Pt}-\mathrm{H}_{2}$ electrodes and of two solutions of a different $\left[\mathrm{H}^{+}\right],\left(\mathrm{h}_{1}\right.$ and $h_{2}$ ) then its E.M.F. is:

$$
\mathrm{E}=\frac{\mathrm{RT}}{\mathrm{F}} \ln \frac{\mathrm{h}_{1}}{\mathrm{~h}_{2}}
$$


$h_{1}$ being known, then $h_{2}$ can be found by determining $E$. The application of this principle in practice will be discussed in detail when the methods of determination of $\left[\mathrm{H}^{+}\right]$are described later.

But at present it is always assumed that the $\mathrm{H}_{2}$-pressure at both electrodes is the same. When such is not the case, then the theory of gas chain requires certain amplifications. In such a case an E.M.F. is developed, even when the $\left[\mathrm{H}^{+}\right]$on both sides is the same. The original equation for concentration chains

$$
\mathrm{E}=\frac{\mathrm{RT}}{\mathrm{F}} \ln \frac{\mathrm{c}_{1}}{\mathrm{C}}-\frac{\mathrm{RT}}{\mathrm{F}} \ln \frac{\mathrm{c}_{2}}{\mathrm{C}}
$$

may assume the simplified form of

$$
\mathrm{E}=\frac{\mathrm{RT}}{\mathrm{F}} \ln \frac{\mathrm{c}_{1}}{\mathrm{c}_{2}}
$$

only when the constant $\mathrm{C}$ has the same value in both terms on the right side of the equation. But this is not true when the gas pressure varies, i.e., the potential of a gas electrode also depends upon the gas pressure about it. Our problem now is to measure the E.M.F. of a gas chain in which the $\left[\mathrm{H}^{+}\right]$is the same throughout, but in which the pressure of hydrogen gas at the two electrodes is different. The platinum electrodes are in equilibrium with two hydrogen atmospheres whose respective partial pressures are $p_{1}$ and $p_{2}$ : the solution in which they are immersed is the same on both sides and of the same $\left[\mathrm{H}^{+}\right]$designated by $h$. On closing the circuit we observe that on the side of the higher partial pressure $\mathrm{H}_{2}$-gas goes into solution as $\mathrm{H}^{+}$-ions and on the other side gaseous hydrogen is formed. If we permit the current to pass long enough until $1 \mathrm{~mol}$ of $\mathrm{H}_{2}$-gas disappears on the left and as much is formed on the right side, then the change of the state of the system is the same as would occur when $1 \mathrm{~mol}$ of $\mathrm{H}_{2}$-gas passes from a vessel in which it was under a pressure of $p_{1}$ into another whose pressure is $p_{2}$. And the work performed is hence equal to the work yielded by a gas, when $1 \mathrm{~mol}$ of it expands from a pressure $p_{1}$ to a lesser pressure $p_{2}$, or to the work $W=R T \ln \frac{p_{1}}{p_{2}}$. The electric work resulting in the same final state of the system is equal to the product of the electromotive force $\mathrm{E}$ by the number of transported coulombs. Since $1 \mathrm{~mol}$ of hydrogen 
amounts to 2 gram-ions of $\mathrm{H}^{+}$, the number of coulombs transported in this case will amount to $2 \mathrm{~F}$. Therefore,

$$
2 \mathrm{~F} \cdot \mathrm{E}=\mathrm{RT} \ln \frac{\mathrm{p}_{1}}{\mathrm{p}_{2}}
$$

and

$$
\mathrm{E}=\frac{\mathrm{RT}}{2 \mathrm{~F}} \ln \frac{\mathrm{p}_{1}}{\mathrm{p}_{2}}
$$

or

$$
\mathrm{E}=\frac{0.0001983}{2 \mathrm{~F}} \log \frac{\mathrm{p}_{1}}{\mathrm{p}_{2}} \text { volts }
$$

Table 23 gives the values of $\mathrm{E}$ of a chain in which the ratio of the two partial pressures is $1: n$.

TABLE 23

\begin{tabular}{l|c|c}
\hline \multirow{2}{*}{$1: n$} & \multicolumn{2}{|c}{ E in millivolts } \\
\cline { 2 - 3 } & At $18^{\circ}$ & At $37^{\circ}$ \\
\hline $1: 1$ & 0 & 0 \\
$1: 1.05$ & 0.6 & 0.6 \\
$1: 1.1$ & 1.2 & 1.2 \\
$1: 1.2$ & 2.3 & 2.4 \\
$1: 1.5$ & 5.1 & 5.4 \\
$1: 2$ & 8.7 & 9.2 \\
$1: 10$ & 28.9 & 30.8 \\
$1: 100$ & 57.7 & 61.5 \\
\hline
\end{tabular}

By "pressure of $\mathrm{H}_{2}$-gas" we always mean the partial pressure. Thus, for instance, in an atmosphere of hydrogen saturated with water vapor by $\mathrm{H}_{2}$-pressure we mean only the partial pressure of bydrogen and not that of the water vapor.

These "pressure-chains" are of interest in the practice of $\mathrm{pH}$ determinations, for the pressure of the atmosphere of hydrogen in the electrode vessels depends in the first place upon the barometric pressure, and, secondly, because of its continuous saturation with water vapor, also upon the temperature.

Gases which form reversible electrodes on platinum surfaces for their own ionic species are primarily hydrogen and chlorine. Oxygen 
is also electromotively active, and at high temperatures (over $300^{\circ}$ ) and by employing a solid electrolyte (glass) instead of an electrolyte solution, such $\mathrm{O}_{2}$-electrode behaves, as Haber ${ }^{1}$ found, exactly as a $\mathrm{OH}^{-}$-ion yielding electrode would lead us to expect. In other words, a chain consisiting of a $\mathrm{H}_{2^{-}}$and of an $\mathrm{O}_{2}$-electrodes, both immersed into one and the same liquid of any composition, the so-called oxyhydrogen gas chain, has that E.M.F. which can be calculated from the chemical affinity of the reaction of the explosive mixture of oxygen and hydrogen. At lower temperatures, however, the usual oxy-hydrogen gas electrode shows a poorly adjusted potential which is about 0.3 volt too low. Otherwise one could determine $\mathrm{OH}^{-}$concentrations with an $\mathrm{O}_{2}$ electrode, just as we determine the $\mathrm{H}^{+}$concentrations with an $\mathrm{H}_{2}$-electrode. But in reality this is not possible. Even though Barendrecht ${ }^{2}$ claims the opposite, he disagrees with all other investigators. As a cause of this irregular behavior of the $\mathrm{O}_{2}$-electrode it is assumed that in the presence of oxygen the platinum is covered by a thin layer of an oxide; hydrogen, on the other hand, reduces this layer and, therefore, the $\mathrm{H}_{2}$-electrode behaves correctly from the standpoint of theory.

\section{The electromotive series}

The potential of a metal against a solution is determined by the nature of the electrode metal as well as by the concentration of the corresponding ions in the solution. Up to this point we have assumed the nature of the electrode metal as being constant and we have discussed only the effect of the concentration. Now we shall briefly deal with the effect of the nature of the metal. The various electrolytic solution tensions of the metals could be characterized by giving that concentration of ions against which the metal shows zero potential. But since the absolute value of single potentials cannot be very accurately determined, it is better to select another way. That potential difference which exists between a metal and a normal solution of its ions is arbitrarily assumed to be standard. Among such standards that of any one metal may be arbitrarily set equal to 0 . At the suggestion made by Nernst we designate the potential

${ }^{1}$ F. Haber, Ann. d. Physik [4]. 26, 927 (1908); Idem., Thermodynamics of Technical Gas Reactions. London 1908.

${ }^{2}$ H. P. Barendrecht, Akad. Wetensch. Amsterdam 27, 1113, 1236, 1406; 28,23 (1919). 
difference between a hydrogen electrode $\left(\mathrm{Pt}-\mathrm{H}_{2}\right)$ under one atmosphere pressure of hydrogen-gas and a solution normal with respect to the $\mathrm{H}$-ion as equal to zero. In this way it was possible to arrive at the electromotive series of the metals and some non-metals shown in table 24, which could be used just as hydrogen in the form of a platinum electrode covered with gas. ${ }^{3}$

With the help of Nernst's equation and these values one can calculate the potential between a metal and a solution containing ions of this metal in any concentration. Conversely, the E.M.F. of the concentration chain being known, the unknown concentration

TABLE 24

Elcctrolytic solution tension at $25^{\circ} \mathrm{C}$.

\begin{tabular}{|c|c|c|c|c|c|}
\hline $\mathrm{Li}^{+}$ & -3.305 & $\mathrm{Fe}^{++}$ & -0.43 & $\mathrm{Pt}^{++}$ & +0.863 \\
\hline $\mathrm{Rb}^{+}$ & -3.205 & $\mathrm{Tl}^{+}$ & -0.32 & $\mathrm{Au}^{+}$ & +1.5 \\
\hline $\mathrm{K}^{+}$ & -3.203 & $\mathrm{Co}^{++}$ & -0.29 & & \\
\hline $\mathrm{Na}^{+}$ & -2.993 & $\mathrm{Ni}^{++}$ & -0.22 & $\mathrm{HSO}_{4}^{-}$ & +2.6 \\
\hline $\mathrm{Ba}^{++}$ & -2.7 & $\mathrm{~Pb}^{++}$ & -0.12 & $\mathrm{SO}_{4}=$ & +1.9 \\
\hline $\mathrm{Sr}^{++}$ & -2.767 & $\mathrm{H}^{+}$ & \pm 0.00 & $\mathrm{O}=$ & $+0.43^{*}$ \\
\hline $\mathrm{Ca}^{++}$ & -2.55 & $\mathrm{As}^{+++}$ & +0.292 & $\mathrm{OH}^{-}$ & $+0.88 \dagger$ \\
\hline $\mathrm{Mg}^{++}$ & -1.55 & $\mathrm{Cu}^{++}$ & +0.34 & $\mathrm{Cl}^{-}$ & +1.35 \\
\hline $\mathrm{Al}^{+++}$ & -1.276 & $\mathrm{Bi}^{+++}$ & +0.391 & $\mathrm{Br}^{-}$ & +1.08 \\
\hline $\mathrm{Mn}^{++}$ & -1.075 & $\mathrm{Hg}^{+}$ & +0.86 & $\mathrm{I}^{-}$ & +0.54 \\
\hline $\mathrm{Zn}^{++}$ & -0.766 & $\mathrm{Ag}^{+}$ & +0.8 & $\mathrm{~F}^{-}$ & +1.9 \\
\hline $\mathrm{Cd}^{++}$ & -0.40 & $\mathrm{Pd}^{++}$ & +0.789 & $\mathrm{~S}=$ & -0.55 \\
\hline $\mathrm{Sn}^{++}$ & -0.1 & & & $\mathrm{CH}_{3} \mathrm{CO}$ & +2.5 \\
\hline
\end{tabular}

* $\dagger$ These two values are in reference to a solution $1 \mathrm{~N}$ with respect to $\mathrm{OH}^{-}-$ ions. They are frequently given in tables in reference to a $1 \mathrm{NH}^{+}$solution, in which case they are +1.23 and +1.68 respectively.

of the metal ions in the solution can be calculated. This is particularly important for the cases where complex ions are formed and of the relatively insoluble metal salts. Thus, for example, the solubility of $\mathrm{AgCl}$ can be estimated by determining the concentration of the $\mathrm{Ag}^{+}$-ions in the solution in the chain

$$
\mathrm{Ag}\left|\begin{array}{c}
\mathrm{AgNo}_{3} \\
1 \text { molar }
\end{array}\right| \begin{gathered}
\mathrm{AgCl}, \text { sat. } \\
\text { solution }
\end{gathered} \mid \mathrm{Ag}
$$

${ }^{3}$ Some of these values were not determined in the direct experimental way, but were indirectly calculated. 
When $\mathrm{KCN}$ is added to a solution of a silver salt, a complex AgCN ion is formed and the $\mathrm{Ag}^{+}$-ions disappear from the solution except for an extremely minute residue. This residue can also be estinated by means of the concentration chain.

50a. Oxidation-Reduction Potentials and the Quinhydrone Electrode.

A. Theory. In recent years, since the appearance of the last German edition of this book oxidation-reduction potentials have assumed considerable importance, a brief outline of the underlying theory will be given here as an addendum only.

By the term oxidation we understand either:

1. The addition of oxygen (or of an OHi group), or the loss of hydrogen, or,

2. The gain of a positive charge, or the loss of a negative charge.

These four processes are obviously quite different in nature, but their consequences may be identical, so that frequently it cannot be discriminated by which one of these four possible ways the end result of oxidation has been achieved. Thus, for example, in the reaction between metallic sodium and water,

$$
\mathrm{Na}+\mathrm{H}_{2} \mathrm{O} \rightarrow \mathrm{NaOH}+\frac{1}{2} \mathrm{H}_{2} \rightarrow \mathrm{Na}^{+}+\mathrm{OH}^{-}+\frac{1}{2} \mathrm{H}_{2}
$$

the oxidation of the sodium may be interpreted either in terms of an addition of oxygen ( $\mathrm{OH}$ group) to the sodium, or, as an addition of a positive charge to the metallic sodium at the expense of the $\mathrm{H}^{+}$ ions of the dissociated $\mathrm{H}_{2} \mathrm{O}$ molecules.

Reduction is exactly the reverse process to that of oxidation and needs no further elucidation.

There are some types of oxidation and reduction which in practice can be made to occur with any reasonable velocity only by means of an exchange of electric charges.

If we have in solution simultaneously a substance and its oxidation product, but no other substances which could act either as oxidizing of reducing agents for the original pair of substances, nothing will happen. For example, in a solution containing $\mathrm{Fe}^{++}$and $\mathrm{Fe}^{+++}$ salts the following reaction is conceivable:

$$
\mathrm{Fe}^{++}+\mathrm{Fe}^{+++} \rightleftharpoons \mathrm{Fe}^{+++}+\mathrm{Fe}^{++}
$$

This reaction, however, will have no discernable consequences, since as much of $\mathrm{Fe}^{++}$disappears as is formed, and the same applies to the $\mathrm{Fe}^{+++}$. 
But if into the same solution we immerse such a chemically indifferent electrode as one of platinum or of gold and make it a part of a closed circuit, the $\mathrm{Fe}^{++}$ions may receive an additional charge (be oxidized) from the current, while the $\mathrm{Fe}^{+++}$may give up one of its charges (be reduced) to the current. According as to whether the reaction

$$
\mathrm{Fe}^{++}+\oplus \rightleftharpoons \mathrm{Fe}^{+++}
$$

depending chiefly upon the concentration ratio, tends to occur predominatingly in the one or in the other direction, an increase of the oxidation or of the reduction product will result.

The chemical force with which the above oxidation-reduction reaction tends to occur in the one and in the other direction depends upon the specific chemical nature of the process involved and, secondly, upon the concentrations. There is a definite concentration ratio at which the forces tending towards oxidation and towards reduction are mutually compensating. If we designate this particular concentration ratio by $c_{0}$, then it can be stated that oxidation will predominate when $\frac{\left[\mathrm{Fe}^{+++}\right]}{\left[\mathrm{Fe}^{++}\right]}>c_{0}$ while the reduction process will prevail when $\frac{\left[\mathrm{Fe}^{+1+}\right]}{\left[\mathrm{Fe}^{++}\right]}<\mathrm{c}_{0}$

- Let us now take a galvanic chain:

$$
\mathrm{Pt}\left|\begin{array}{l}
{\left[\mathrm{Fe}^{+++}\right]=\mathrm{c}_{1}} \\
{\left[\mathrm{Fe}^{++}\right]=\mathrm{c}_{1}{ }_{1}}
\end{array}\right| \begin{aligned}
& {\left[\mathrm{Fe}^{+++}\right]=\mathrm{c}_{2}} \\
& {\left[\mathrm{Fe}^{++}\right]=\mathrm{c}_{2}^{\prime}}
\end{aligned} \mid \mathrm{Pt}
$$

Upon closing the chain with a metallic conductor between the two electrodes, an electric current will generally be produced in this circuit, let us say frem left to right within the chain, hence from right to left in the external circuit. We shall also assume that the volumes of the two solutions are sufficiently great, so that no appreciable change in their concentrations will occur on the passage of a small current. When 1 faraday (96500 coulombs) had flown through any cross section of this circuit, the electric work $A=F \pi$ will result, where $\pi$ is the E.M.F. The same effect which had been produced by the current, could result from an osmotic transport in which one mol of $\mathrm{Fe}^{+++}$in concentration $\mathrm{c}_{1}$ is isothermally and reversibly compressed to the concentration $c_{2}$, which would entail the work, $\mathrm{A}=$ $\operatorname{RTln} \frac{\mathrm{c}_{2}}{\mathrm{c}_{1}}$, and at the same time $1 \mathrm{~mol}$ of $\mathrm{Fe}^{++}$is correspondingly diluted 
from $\mathrm{c}_{2}^{\prime}$ to $\mathrm{c}_{1}^{\prime}$ the work amounting to $\mathrm{RT} \ln \frac{\mathrm{c}_{1}^{\prime}}{\mathrm{c}^{\prime}{ }_{2}}$. But since the electric work of the current and the osmotic work are maximal, they must be equal to each other, hence

$$
\mathrm{F} \pi=\mathrm{RT} \ln \frac{\mathrm{c}_{2}}{\mathrm{c}_{1}}+\mathrm{RT} \ln \frac{\mathrm{c}_{1}^{\prime}}{\mathrm{c}_{1}^{\prime}}
$$

or,

$$
\pi=\mathrm{E} \cdot \mathrm{M} \cdot \mathrm{F} \cdot=\frac{\mathrm{RT}}{\mathrm{F}} \ln \frac{\mathrm{c}_{2} \mathrm{c}_{1}^{\prime}}{\mathrm{c}_{1} \mathrm{c}^{\prime}{ }_{2}}
$$

when $\frac{\mathrm{c}_{1}^{\prime}}{\mathrm{c}_{2}^{\prime}}=\mathrm{C}_{0}$, then

$$
\text { E.M.F. }=\frac{R T}{F} \ln \frac{c_{2}}{c_{1}}+\frac{R T}{F} \ln C_{0}=\frac{R T}{F} \ln \frac{c_{2}}{c_{1}}+K
$$

where $\mathrm{K}$ is a constant characterizing the given chemical reaction. Therefore equation (1) represents also the potential of a single $\mathrm{Fe}^{+++}$ $-\mathrm{Fe}^{++}$electrode, in which equation an additive constant is left undetermined.

B. The Quinhydrone Electrode-An Application.

When an incompletely dissociated electrolyte participates in such a reaction as outlined above, some special aspects arise which may be developed into a very useful practical application, as in the case of the quinonehydroquinone electrode.

Hydroquinone, $\mathrm{C}_{6} \mathrm{O}_{2} \mathrm{H}_{6}$, is a very weak dibasic acid. Quinone, $\mathrm{C}_{6} \mathrm{O}_{2} \mathrm{H}_{4}$, on the other hand, is practically a non-electrolyte. Whenever a mixture of both of those substances is placed in contact with a platinum electrode in a closed circuit the following process takes place:

$$
\underset{\text { Secondary hydroquinone ion }}{\mathrm{C}_{6} \mathrm{O}_{2} \mathrm{H}_{4}^{-}} \rightleftharpoons \underset{\text { Quinone }}{\mathrm{C}_{6} \mathrm{O}_{2} \mathrm{H}_{4}+2} \oplus
$$

In analogy with equation (1) above, the potential of such a single electrode is

$$
\mathbf{E}=\frac{\mathrm{RT}}{2 \mathrm{~F}} \ln \frac{\mathrm{c}^{\prime}}{\mathrm{c}}+\mathrm{K}
$$

where $c^{\prime}$ is the concentration of the quinone and $c$ is the concentration of the secondary hydroquinone ions. The value 2 appears in 
the divisor of the right hand term because the secondary hydroquinone ion is bivalent, and, therefore, the transport of $\mathrm{F}$ coulombs is equivalent to the osmotic transport of $\frac{1}{2} \mathrm{~mol}$.

The ratio of the concentrations of the secondary hydroquinone ions and of the total hydroquinone is regulated by the mass law in the following manner:

$$
\frac{\left[\mathrm{C}_{6} \mathrm{H}_{2} \mathrm{O}_{4}{ }^{-}\right]\left[\mathrm{H}^{+}\right]^{2}}{\left[\mathrm{C}_{6} \mathrm{O}_{2} \mathrm{H}_{6}\right]}=\mathrm{k}
$$

where $\left[\mathrm{C}_{6} \mathrm{H}_{2} \mathrm{O}_{6}\right]$ means the concentration of the undissociated hydroquinone molecules.

When the reaction is not too alkaline, $\mathrm{pH}<$ about 8.5 , so that the primary as well as the secondary ions of the hydroquinone form but an insignificant portion of the total hydroquinone, the concentration of the undissociated hydroquinone is practically equal to that of the total hydroquinone. Hence the last equation may be written

$$
\left[\mathrm{C}_{6} \mathrm{O}_{2} \mathrm{H}_{4}=\right]=\frac{\mathrm{k}\left[\mathrm{C}_{6} \mathrm{O}_{2} \mathrm{H}_{6}\right]}{\left[\mathrm{H}^{+}\right]^{2}}
$$

where $\left[\mathrm{C}_{6} \mathrm{H}_{2} \mathrm{O}_{6}\right]$ means the total concentration.

By substituting (3) into equation (2), we obtain

$$
\mathrm{E}=\frac{\mathrm{RT}}{2 \mathrm{~F}} \ln \frac{\left[\mathrm{C}_{6} \mathrm{O}_{2} \mathrm{H}_{4}\right]\left[\mathrm{H}^{+}\right]^{2}}{\mathrm{k}\left[\mathrm{C}_{6} \mathrm{O}_{2} \mathrm{H}_{6}\right]}+\mathrm{K}
$$

which may be further simplified by substituting $K_{1}$ for $K$, so as to include $k$, thus

$$
\mathrm{E}=\frac{\mathrm{RT}^{\prime} \mathrm{T}}{\mathrm{F}} \ln \frac{\left[\mathrm{C}_{6} \mathrm{O}_{2} \mathrm{H}_{4}\right]\left[\mathrm{H}^{+}\right]}{\left[\mathrm{C}_{6} \mathrm{O}_{2} \mathrm{H}_{6}\right]}+\mathrm{K}_{1}
$$

When in a series of such different electrodes the ratio

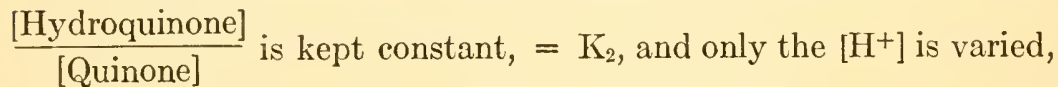
then

$$
\mathrm{E}_{1}=\frac{\mathrm{RT}}{\mathrm{F}} \ln \frac{\left[\mathrm{H}^{+}\right]}{\mathrm{K}_{2}}+\mathrm{K}_{1}=\frac{\mathrm{RT}}{\mathrm{F}} \ln \left[\mathrm{H}^{+}\right]+\mathrm{K}_{3}
$$

This means that the potential depends upon the $\mathrm{pH}$, exactly as it does with hydrogen-gas electrodes. By setting up a chain consisting of two such electrodes in which the ratio $\frac{\text { [Quinone] }}{[\text { Hydroquinone] }}$ is the same, 
but the $\mathrm{pH}$ values are different, one of these being known and the other unknown, the latter may be determined as in the ordinary $\mathrm{H}_{2}$ gas chain. For $\mathrm{pH}$ values $>8.5$ the above deseribed limitation renders the method unusable.

In practice the mixture of quinone-hydroquinone in a constant proportion is replaced by the addition to the solution whose $\mathrm{pH}$ is to be measured of a small amount of quinhydrone. This is a molecular addition compound consisting of $1 \mathrm{~mol}$ hydroquinone $+1 \mathrm{~mol}$ quinone, and which in solution splits into its components.

For a more detailed account of the theory of oxidation-reduction potentials and of the use of the quinhydronc electrode the reader is referred to the original literature. ${ }^{4}$

${ }^{4}$ E. Biilman and collaborators, Ann. de Chim. 15, 109 (1921); Ibid. 16, 321 (1921). Trans. Faraday Soc. 19, Part 3, (1924). Jour. Chem. Soc. 125, 1954 (1924).

S. P. L. Sørensen, M. Sørensen and K. Linderstrøm-Lang. Compt. rend. trav. Iab. Carlsberg, 14, No. 14 (1921); K. Linderstrøm-Lang. Ibicl. 16, No. 3 (1925).

W. M. Clark and collaborators. U. S. Public Health Reports, 38-39, (1923-4).

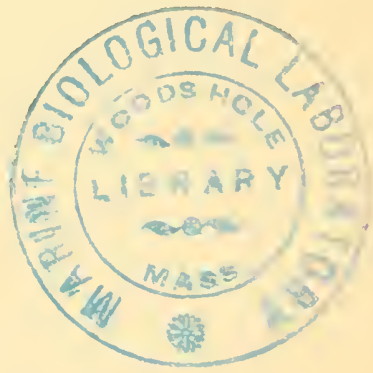




\section{CHAPTER VII}

\section{Diffusion Potentials (Potential Differences at Liquid JunCTIONS)}

\section{SUMMARY OF CONTENTS}

Potential differences arise at the junction of two aqueous solutions which differ in kind or in the concentration of the dissolved electrolytes. These can be explained and calculated on the basis of Nernst's diffusion theory. The calculation is simple in a few special cases, but in general it is difficult. Since these potentials are of little signiflcance in the realm of physiology, and since they can, in experimental technic, be readily avoided, their calculation may well be omitted in this work. The most suitable method of preventing diffusion potentials is through the use of a salt bridge consisting of a saturated $\mathrm{KCl}$ solution.

51. When a solution has in different places a varying content of electrolytes a potential difference arises between the places of different composition. The difference in composition may either be one of concentration of the same electrolyte or one of the kind of electrolyte involved; or it may consist of both of these variations at once. But the solvent is necessarily the same throughout the solution, and, as a rule, it is water. Cases in which this last condition is not fulfilled will be dealt with in another chapter. Nernst furnished an explanation of these potentials on the basis of the ionic theory in the following manner. Let us assume that we have in the left part of a horizontal tube an $\mathrm{HCl}$ solution of the concentration $c_{1}$ and in the right hand part an $\mathrm{HCl}$ solution whose concentration is $c_{2}$. At the junction of these two solutions diffusion occurs, and then a concentration gradient is formed. The relative incline of such a gradient depends upon the difference of the concentrations and also upon the rate of diffusion (or diffusion coefficient) of the diffusing substance. The more rapidly the substance diffuses the smaller the gradient. If two different substances are present in solution, then each develops its own gradient independent of the other. Since $\mathrm{HCl}$ in solution is really a mixture of $\mathrm{H}$ - and $\mathrm{Cl}$-ions, the same would apply in this 
case, if it were not for the hindering effect of the electrostatic attraction operating between these oppositely charged ions. The more rapidly diffusing $\mathrm{H}$-ions would establish a flatter gradient, the $\mathrm{Cl}$-ions a steeper gradient. But the electrostatic attractions prevent this. The $\mathrm{H}$-ions are retarded by the $\mathrm{Cl}$-ions, the $\mathrm{Cl}$-ions are accelerated by the $\mathrm{H}$-ions, and an average diffusion gradient results. It is true that the $\mathrm{H}$-ions are slightly in advance, but only insofar as the potential difference developed here permits it. Consequently the more dilute solution becomes positively charged with respect to the more concentrated one. The amount of such predominating $\mathrm{H}$-ions is extremely minute and is beyond chemical measurement, and it is only recognized through the potential difference which develops under these conditions.

The values of this potential difference can be, according to Nernst, ${ }^{1}$ calculated in the following way. Let us recall the two ends of the tube the left end of which contains $\mathrm{HCl}$ in concentration $\mathrm{c}_{1}$, and the right in concentration $c_{2}$. Now these two ends are connected by an external metal conductor. The immersed electrodes in this case are assumed to have, for the sake of simplicity, equal and opposite potential differences with respect to the solutions and thus counterbalance each other. Then the E.M.F. of this chain is simply equal to the diffusion potential $\pi$. The circuit is now kept closed until $\mathrm{F}$ coulombs have passed through the transverse section of the tube. Then the electric work $\pi \times \mathrm{F}$ has been accomplished in a reversible manner. ${ }^{2}$ The effect of this work is that a certain amount of $\mathrm{H}$-ions migrated from the stronger into the weaker solution and a certain amount of $\mathrm{Cl}$-ions migrated in the opposite direction; in fact a total of 1 gram-ions had been transported. If the migration-velocity of the $\mathrm{H}$-ions $=\mathrm{u}$, and that of the Cl-ions $=\mathrm{v}$, then of $1 \mathrm{~mol}$ of ions the fractions $\frac{u}{u+v}$ of $\mathrm{H}$-ions and $\frac{v}{u+v}$ of $\mathrm{Cl}$-ions have migrated. The osmotic work to be obtained when $1 \mathrm{~mol}$ of $\mathrm{H}$-ions passes from a solution of concentration $c_{1}$ into one of concentration $c_{2}$ is $=$ RT $\ln \frac{c_{1}}{c_{2}}$; hence of $\frac{u}{u+v}$ moles H-ions the work is $\frac{u}{u+v} \operatorname{RT} \ln \frac{c_{1}}{c_{2}}$, and

${ }^{1}$ W. Nernst, Zeitschr. f. physikal. Chem. 2, 611 (1888); and especially in Ibid. 4, 129 (1889).

${ }^{2}$ On the assumption that the period of time consumed in this experiment is so short that the displacement of substances in the solution through mere diffusion can be neglected. 
correspondingly for the Cl-ions it is $=\frac{v}{u+v} R T \ln \frac{c_{1}}{c_{2}}$. The total osmotic work derived would then be $\frac{u-v}{u+v} R T \ln \frac{c_{1}}{c_{2}}$. Since the amount of the maximal work to be derived depends only upon the initial and final states of the system, regardless of the way or method of procedure, as long as it functions reversibly, it follows that $\pi \mathrm{F}=$ $\frac{\mathrm{u}-\mathrm{v}}{\mathrm{u}+\mathrm{v}} \mathrm{RT} \ln \frac{\mathrm{c}_{1}}{\mathrm{c}_{2}}$.

By transposing $\mathrm{F}$ to the right side and by making the numerical transformations shown on page 162, we obtain:

$$
\pi=\frac{\mathrm{u}-\mathrm{v}}{\mathrm{u}+\mathrm{v}} \times 0.00019 S 3 \mathrm{~T} \log \frac{\mathrm{c}_{1}}{\mathrm{c}_{2}} \text { volts }
$$

This formula agrees quite well with experimental findings. Thus the difference between the migration velocities of the cation and anion is very important in determining the value of $\pi$. Table 25 , taken from Kohlrausch, gives the migration velocities of ions at $18^{\circ} \mathrm{C}$.

TABLE 25

\begin{tabular}{|c|c|c|c|}
\hline \multicolumn{2}{|l|}{$\mathbf{u}=$} & \multicolumn{2}{|l|}{$\mathrm{v}=$} \\
\hline 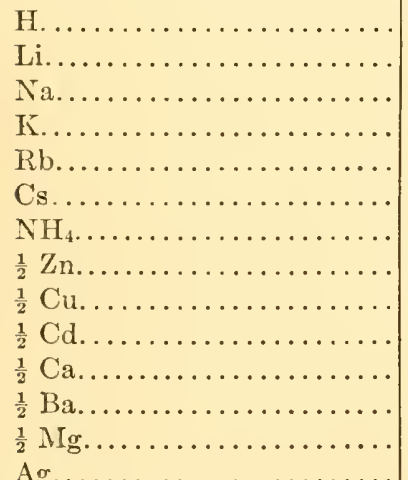 & $\begin{array}{l}318 \\
33.44 \\
43.55 \\
64.67 \\
67.5 \\
68 . \\
64 . \\
46 . \\
46 . \\
46 . \\
51 . \\
55 . \\
45 . \\
540\end{array}$ & 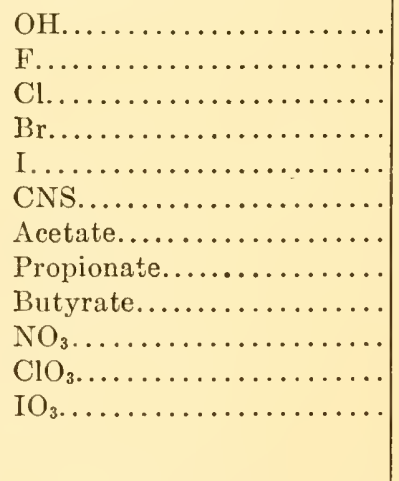 & $\begin{array}{l}174 . \\
46.6 \\
65.44 \\
67.0 \\
66.5 \\
56.6 \\
35 \\
35 . \\
31 . \\
61.78 \\
64 . \\
49 .\end{array}$ \\
\hline
\end{tabular}

TABLE 26

$$
\begin{aligned}
& \mathrm{HCl}+0.0378 \text { volts } \\
& \mathrm{NaOH}-0.0346 \text { volts } \\
& \mathrm{HOH}-0.0265 \text { volts }
\end{aligned}
$$

$$
\begin{array}{ll}
\mathrm{LiCl} & -0.0187 \text { volts } \\
\mathrm{NaCl} & -0.0116 \text { volts } \\
\mathrm{KCl} & -0.0004 \text { volts } \\
\mathrm{NH} & \mathrm{Cl}-0.0007 \text { volts } \\
\mathrm{NH}_{4} \mathrm{NO}_{3}+0.0011 \text { volts }
\end{array}
$$


It can readily be seen from this table that large values for $\mathrm{u}-\mathrm{v}$ can only result, when one of the ions is either a $\mathrm{H}$-ion or an OH-ion. Hence, the diffusion potential of two solutions of a binary electrolyte whose concentrations are in the ratio of $1: 10$ have been calculated for $18^{\circ} \mathrm{C}$. to be as shown in table 26 .

This method of calculation is applicable only when two solutions of differing concentrations of one and the same electrolyte are in contact. For all other cases, the calculation of the diffusion potential is much more complicated.

The presentation of equations generally applicable to any solutions containing any mixtures of electrolytes is a difficult and a still unsolved problem. For the case where two solutions are in contact, one of which contains one electrolyte in concentration $c_{1}$ (and the velocities of its cations and anions are $u_{1}$ and $v_{1}$ ), and the other contains a different electrolyte in concentration $c_{2}$ (whose ions have the velocities of $u_{2}$ and $v_{2}$ ), the following general equation for $\pi$ has been derived by $\mathrm{Planck}^{3}$ :

$$
\pi=0.00019 \$ 3 \mathrm{~T} \log \zeta
$$

where $\zeta$ is defined by the following theoretical equation:

$$
\frac{\zeta \mathrm{c}_{2} \mathrm{u}_{2}-\mathrm{c}_{1} \mathrm{u}_{1}}{\mathrm{c}_{2} \mathrm{v}_{2}-\zeta \mathrm{v}_{1} \mathrm{c}_{1}}=\frac{\log \frac{\mathrm{c}_{2}}{\mathrm{c}_{1}}-\log \zeta}{\log \frac{\mathrm{c}_{2}}{\mathrm{c}_{1}}+\log \zeta} \cdot \frac{\zeta \mathrm{c}_{2}-\mathrm{c}_{1}}{\mathrm{c}_{2}-\zeta \mathrm{c}_{1}}
$$

When the concentrations $c_{1}$ and $c_{2}$ are equal to each other, then the calculation is simplified considerably. Then, as can be readily demonstrated, the value $\zeta$ is easily calculated, and, for two solutions of two different binary univalent electrolytes of the same concentration Planck's formula becomes

$$
\pi=0.0001983 \mathrm{~T} \log \frac{\mathrm{u}_{1}+\mathrm{v}_{2}}{\mathrm{u}_{2}+\mathrm{v}_{1}}
$$

where $\mathrm{u}_{1}$ and $\mathrm{v}_{1}$ are the velocities of the cation and anion, respectively of the one electrolyte, and, correspondingly, $u_{2}$ and $v_{2}$ for the other electrolyte. Thus from this formula we obtain, for example, the

${ }^{3}$ Planck, Wiedemanns Annalen 40, 561 (1890). 
figures in table 27 (for $\pi$ ) for solutions of equal concentrations at $18^{\circ} \mathrm{C}$.

When more than one electrolyte are present in each of the two solutions the following substitutions must be made in the above formulas:

$$
\begin{aligned}
& \mathrm{u}_{1} \mathrm{c}_{1} \text { substituted by } \mathrm{u}_{1}{ }^{\prime} \mathrm{c}_{1}{ }^{\prime}+\mathrm{u}_{1}{ }^{\prime \prime} \mathrm{c}_{1}{ }^{\prime \prime}+\ldots \\
& \mathrm{u}_{2} \mathrm{c}_{2} \text { substituted by } \mathrm{u}_{2}{ }^{\prime} \mathrm{c}_{2}{ }^{\prime}+\mathrm{u}_{2}{ }^{\prime \prime} \mathrm{c}_{2}{ }^{\prime \prime}+\ldots \\
& \mathrm{v}_{1} \mathrm{c}_{1} \text { substituted by } \mathrm{v}_{1}{ }^{\prime} \mathrm{c}_{1}{ }^{\prime}+\mathrm{v}^{\prime \prime} \mathrm{c}_{2}{ }^{\prime \prime}+\ldots \\
& \mathrm{v}_{2} \mathrm{c}_{2} \text { substituted by } \mathrm{v}_{2}{ }^{\prime} \mathrm{c}_{2}{ }^{\prime}+\mathrm{v}_{2}{ }^{\prime \prime} \mathrm{c}_{2}{ }^{\prime \prime}+\ldots
\end{aligned}
$$

where the subscripts 1 and 2 denote, as before, the two solutions in contact, while the superscripts $\left({ }^{\prime},{ }^{\prime}\right.$, etc.) refer to the different kinds of ions.

Planck's equations (the first, general one, as well as the second, special one) apply to the case where all electrolytes consist of two

TABLE 27

$\begin{array}{lllr}\mathrm{HCl}, \mathrm{NaCl} & 0.0315 \text { volts } & \mathrm{NaCl}, \mathrm{KCl} & -0.0044 \text { volts } \\ \mathrm{HCl}, \mathrm{KCl} & 0.0268 \text { volts } & \mathrm{NH}_{4} \mathrm{NO}_{3}, \mathrm{KCl} & 0.0006 \text { volts }\end{array}$

$\mathrm{NaOH}, \mathrm{NaCl}-0.0173$ volts

$\mathrm{KOH}, \mathrm{NaCl}-0.0127$ volts

univalent ions. When all the ions present are n-valent then the potential amounts to $\frac{1}{n}$ th of its value as shown by the equation. But in cases where ions of different valences occur the calculation assumes great difficulties which have not yet been solved.

The condition on which Planck based his calculations is that the two solutions which are in contact should not be mechanically mixed and that the above mentioned temporarily stationary concentration gradient had been established.

P. Henderson ${ }^{4}$ making a somewhat different assumption concerning the connecting boundary between the two solutions, deduced for certain conditions an equation which has the advantage over Planck's of not being theoretically transcendental. Henderson assumed that the connecting boundary is not the result of spontaneous diffusion of the two solutions in contact, but that it ensues through a

${ }^{4}$ P. Henderson, Zeitschr. f. physikal. Chem. 59, 118 (1907); 63, 325 (1908). 
continuous mechanical process of mixing between the two solutions in such a way that the composition of solution I gradually passes through the intermediate mixture zone into the composition of the solution II. The assumption for the temporary constancy of such a potential is that the two solutions have at least one ion species in common. The Henderson equation is

$$
\pi=0.0001983 \cdot \mathrm{T} \cdot \frac{\left(\mathrm{U}_{\mathrm{I}}-\mathrm{V}_{\mathrm{I}}\right)-\left(\mathrm{U}_{\mathrm{II}}-\mathrm{V}_{\mathrm{II}}\right)}{\left(\mathrm{U}_{1}^{\prime}+\mathrm{V}_{\mathrm{I}}{ }^{\prime}\right)-\left(\mathrm{U}_{\mathrm{II}}{ }^{\prime}+\mathrm{V}_{\mathrm{II}}{ }^{\prime}\right)} \log \frac{\mathrm{U}_{\mathrm{I}}{ }^{\prime}+\mathrm{V}_{\mathrm{I}}{ }^{\prime}}{\mathrm{U}_{\mathrm{II}}{ }^{\prime}+\mathrm{V}_{\mathrm{II}}{ }^{\prime}}
$$

in which

$$
\begin{aligned}
& \mathrm{U}_{\mathrm{I}}=\mathrm{u}_{1} \mathrm{c}_{1}+\mathrm{u}_{2} \mathrm{c}_{2}+\mathrm{u}_{3} \mathrm{c}_{3} \ldots \\
& \mathrm{V}_{\mathrm{I}}=\mathrm{v}_{1} \overline{\mathrm{c}}_{1}+\mathrm{v}_{2} \overline{\mathrm{c}}_{2}+\mathrm{v}_{3} \mathrm{c}_{3} \\
& \mathrm{U}_{\mathrm{I}}^{\prime}=\mathrm{u}_{1} \mathrm{w}_{1} \mathrm{c}_{1}+\mathrm{u}_{2} \mathrm{w}_{2} \mathrm{c}_{2}+\mathrm{u}_{3} \mathrm{w}_{3} \mathrm{c}_{3} \ldots \\
& \mathrm{V}_{\mathrm{I}}^{\prime}=\mathrm{v}_{1} \overline{\mathrm{W}}_{1} \overline{\mathrm{c}}_{1}+\mathrm{v}_{2} \overline{\mathrm{W}}_{2} \overline{\mathrm{c}}_{2}+\mathrm{v}_{3} \overline{\mathrm{W}}_{3} \overline{\mathrm{c}}_{3}
\end{aligned}
$$

where $\mathrm{c}=$ the concentration (for a negative ion, $\overline{\mathrm{c}}$ ).

$\mathrm{u}=$ the mobility of a cation and $\mathrm{v}$ of an anion.

$\mathrm{w}=$ the valence ( $\overline{\mathrm{w}}$ for an anion).

The subscripts I, II refer to the two solutions, the subscripts $1,2,3$, etc., to the different ion species.

This equation was further modified by Cumming. ${ }^{5}$

Planck's and Henderson's equations do not yield the same values, except under certain conditions, such as, for instance, when each of the two solutions contains but one salt, in the same concentration, and both having one ion species in common. It is to be expected, therefore, that the diffusion potential should in general represent a somewhat fluctuating value depending on whether Planck's or Henderson's conception of the contact zone is assumed. Indeed, $\mathrm{Chanoz}^{6}$ has shown that the diffusion potential changes with time. Thus when a chain is arranged of the type

Electrode || Solution I | Solution II | Solution I || Electrode A

$\mathrm{B}$

we should expect that its E.M.F. would be equal to zero, for here not only the electrode potentials but also the diffusion potentials

${ }^{5}$ Cumming, Transact. Faraday Soc. 8, 86 (1912).

${ }^{6}$ Chanoz, Ann. de l'univ. Lyon, Nouv. Sér. 1, 18 (1906), eited from P. Henderson. 
at $\mathrm{A}$ and $\mathrm{B}$ disappear. But if the zone of contact at $\mathrm{A}$ were old and the one at $B$ freshly renewed, then a potential difference of up to six millivolts will develop, which will gradually diminish on standing. This fluctuation of the potential with time has also been found by a number of investigators. ${ }^{7}$ Recently an ingenious apparatus has been devised by Lamb and Larson ${ }^{8}$ by means of which they succeeded in maintaining a constantly self renewing fresh junction of the solutions, and they obtained potential values of remarkable reproducibility. Without some such arrangement it can scarcely be even expected to attain the sharply defined boundary surfaces which were assumed by Planck. And since the time effect must be such that the potential gradually diminishes, in practice one frequently finds values for potentials which are smaller than those calculated according to Planck's equation. Recently Fales and Vosburgh (1. c.) have even stated that between a saturated $(4.1 \mathrm{~N}) \mathrm{KCl}$ solution and 0.1 to 1.0 $N \mathrm{HCl}$-solution there arises no measurable diffusion potential at all.

Under these circumstances, it must be said that the value of a diffusion potential is not as sharply defined as it should unconditionally be required in concentration chain measurements for analytical purposes, for which it is frequently employed. For this reason it is less important in the practical use of concentration chains to calculate exactly the diffusion potential than it is to arrange the chain in such a way that the diffusion potential should become extremely small and negligible. The method of achieving this purpose will be described as follows:

All these diffusion potentials originate in the differences among ion mobilities. Between electrolytes whose individual ions have equal mobilities no diffusion potential develops. The electrolytes in which the difference between the values of $\mathrm{u}$ and $\mathrm{v}$ is the smallest are $\mathrm{KCl}(64.6$ and 65.5$)$ and a few others such as $\mathrm{NH}_{4} \mathrm{NO}_{3}(64$ and 61.7). Solutions of different concentrations of $\mathrm{KCl}$ or those of $\mathrm{NH}_{4}$ $\mathrm{NO}_{3}$ have therefore extremely small diffusion potentials. Thus

${ }^{7}$ A. Weyl, Messung von Diffusionspotentialen konzentrierter Chloridlösungen. Dissert. 1905. Bjerrum, Zeitschr. f. Elektrochem. 17, 58, 389 (1911). G. N. Lewis and F. F. Rupert, Journ. of the Amer. Chem. Soc. 33, 299 (1911); A. C. Cumming and E. Gilchrist, Transact. Faraday Soc. 9, 174 (1913); H. A. Fales and W. C. Vosburgh, Journ. of the Amer. Chem. Soc. 40, 1291 (1918).

8 A. B. Lamb and A. T. Larson, Journ. of the Amer. Chem. Soc. 42, 229 (1920). 
between any two solutions of $\mathrm{KCl}$ there arises no potential of any practical importance, as these calculated figures show:

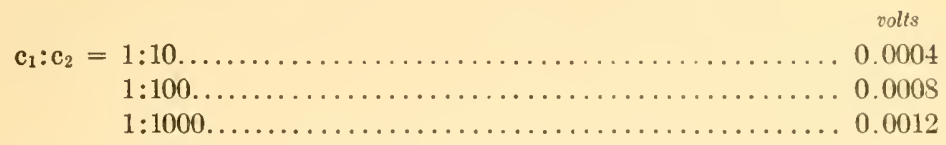

And even these values must surely be much too high for contact boundaries that are not very sharply defined. Hence the diffusion potential arising between any electrolyte solution and a $\mathrm{KCl}$ solution is always smaller than the one with a solution of any other salt in which the mobilities of the cations and anions differ very markedly. Consequently it should be possible to diminish greatly the diffusion potential between any two solutions by interposing between them a $\mathrm{KCl}$ solution. This effect of $\mathrm{KCl}$ increases with the increasing concentration of its solution, since then the conduction is due more and more to the $\mathrm{K}$ - and $\mathrm{Cl}$-ions.

In most cases then it suffices to interpose a saturated $(4.1 \mathrm{~N}) \mathrm{KCl}$ solution in order to practically abolish the diffusion potential.

A wide use is made of this principle in practical concentration chain measurements.

In most cases then the diffusion potential may be neglected when a $\mathrm{KCl}$ solution is used as a liquid bridge. And yet in very acid $(\mathrm{pH}$ $<3$ ) and in very alkaline solutions the abolition of this potential is not quite complete. In such cases Bjerrum's ${ }^{9}$ empirically derived extrapolation method is applied. The E.M.F. of the chain is measured once with the interposition of a $1.75 \mathrm{M} \mathrm{KCl}$ solution and then it is measured again with a $3.5 \mathrm{M} \mathrm{KCl}$ solution. If a difference between these measurements is found, this difference is to be taken into account in order to obtain the approximately true value of the potential difference of the electrodes. For example

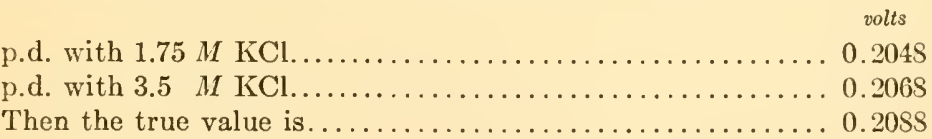

This extrapolation must amount to but a few millivolts, if its claim to certainty is to be assumed. Of all physiological fluids such an extrapolation is taken into consideration only in the gastric juice

${ }^{9}$ N. Bjerrum, Zeitschr. f. physikal. Chem. 53, 428 (1905). 
because of its high acidity. It must scarcely ever exceed 1 to 2 millivolts.

The question is of greater importance in dealing with concentration chains in which strong acids and bases are involved, as for example in the determination of the dissociation constant of water or in the standardization of the normal hydrogen electrode. Here the required extrapolation values are frequently so great that pure acid solutions are never used, but instead we employ acid solutions containing $\mathrm{KCl}$, in order to decrease the diffusion potential. But since this affects the activity of the H-ions, which can still be only approximately calculated, therefore, a practical limitation is hereby imposed upon the attainable accuracy of all $\mathrm{pH}$ determinations. It may be said that such measurements are inaccurate to the extent of at least 0.5 millivolts, which corresponds to a probable error in $\mathrm{pH}$ of about 0.01 .

\section{The physiological significance of diffusion potentials}

It is evident that electrode potentials do not occur in the living organisms, for there are no free metals present. But the problem arises as to whether or not the potential differences observed in the organism could be explained as being diffusion potentials. But it is out of question that diffusion potentials have any considerable share in these tissue potentials. For the potentials that have been observed between injured and uninjured parts are of the order of magnitude of 0.02 to 0.05 volts and even above. Such high diffusion potentials can only develop under extreme conditions such as never occur in the living organism. In the almost always approximately neutral and $\mathrm{NaCl}$-containing tissue fluids not even a diffusion potential of 0.01 volt can ever arise as will be readily seen from the discussion given above. 


\section{CHAPTER VIII}

\section{Potentials at Phase Boundaries}

\section{SUMMARY OF CONTENTS}

At the boundary of two phases there generally arises a potential difference which depends upon the specific distribution of a dissolved electrolyte between the phases. If we assume that every ionic species has a characteristic distribution coefficient, then the coefficients of the cations and of the anions of an electrolyte will not be equal. Hence it follows, in the first place, that the distribution law does not hold for the whole electrolyte when it does hold for its individual ion components, and secondly, that a potential difference arises at the phase boundary and that this potential bears a thermodynamically calculable relation to the electrolytic solution pressures of the various ionic species in each phase. The potential difference can be calculated from the concentrations of any ionic species common to the two phases, and the E.M.F. of a chain involving two such boundaries may vary, according to the conditions, from zero to a certain maximal value. The latter is comparable to that of an ordinary concentration chain with metal electrodes. With respect to their electromotive behavior the different ions as well as the solvents are arranged in a serial sequence. These phase boundary potentials appear to be of great physiological importance, for it is probable that many of the potential differences found in living organisms are of this nature.

\section{The origin and calculation of phase-boundary potentials}

Properly speaking, electrode potentials are phase boundary potentials as well. But here we shall consider as such only those potentials which arise without the participation of metals at phase boundaries. Both phases in the case to be considered shall be solutions-not, as in diffusion potentials, miscible liquids which can become homogeneous through diffusion, but rather immiscible fluids separated by a sharply defined boundary at the place of contact. These phases can exist side by side in complete equilibrium, whereas the diffusion potential is characterized by a lack of equilibrium. 
It will be useful to recall clearly the following points.

A current producing chain can only be represented by a system which, when considered as a whole, is not in a state of chemical equilibrium.

The E.M.F. of such a chain is the algebraic sum of all the single potential differences present between the poles of the chain. Such a single potential is always found between two contiguous layers. These two contiguous layers may coexist in chemical equilibrium (metal electrode potentials, phase boundary potentials, and see below for Donnan's membrane potentials), i.e., the electric force is compensated by an opposing force. Or they may not coexist in a state of equilibrium (diffusion potentials). Two contiguous layers, considered as an isolated chemical system, retain their potential difference as long as they remain in chemical equilibrium (e.g., a single silver electrode immersed into a $\mathrm{AgNO}_{3}$ solution); and they gradually lose their potential difference when the latter depends only upon the lack of equilibrium (e.g., the boundary layers between a concentrated and a dilute $\mathrm{HCl}$ solution which are in contact; when these two solutions become homogeneous through diffusion, the potential difference disappears, without any electric current having been generated).

Two true aqueous solutions never form distinct phases in relation to each other. For this purpose there are required two solutions having immiscible or poorly miscible solvents, e.g., water and oil. As a rule in the following discussion we shall assume an aqueous phase in contact with an "oil" phase as it was designated by Beutner. A better way of stating this would be: two phases of different dielectric constants.

The first fundamental principles for a rational examination of boundary potentials are to be found in a paper by Nernst ${ }^{1}$ who also in this field must be regarded as the founder of the theory; and then also in the equally important investigations of Haber. ${ }^{2}$ The significance of these investigations of Nernst and of Haber for physiological processes had not been recognized for a long time by physiologists, although these authors were fully aware of the far reaching physio-

1 W. Nernst, Zeitschr. f. physikal. Chem. 9, 140 (1892); Riesenfeld, Über elektrolytische Erscheinungen und elektromotorische Kräfte an der Grenze zweier Lösungsmittel. Diss. Göttingen 1901; Nernst and Riesenfeld, Ann. d. Physik. [4] 8, 600 (1902).

${ }^{2}$ F. Haber, Ann. d. Physik. [4] 26, 927 (1908); Haber and Klemensiewicz, Zeitschr. f. physikal. Chem. 67, 385 (1909). 
logical import of their ideas and pointed them out. ${ }^{3} \quad$ R. Beutner ${ }^{4}{ }^{4}$ a former student of Haber's and under the encouragement of Jacques Loeb, has recently given us a substantial development of this problem, which we shall discuss next. But we must also give full credit to the theories of Nernst and Haber. While there are no fundamental differences between them, they are both indispensable.

We shall now show thermodynamically that, in general, between two solutions consisting of immiscible solvents a potential difference must arise, even though they be in a state of chemical equilibrium with respect to each other. Let us, for example, shake up portions of water and of guaiacol or of amyl alcohol with some $\mathrm{HCl}$, so that the electrolyte is distributed in the two solvents and is consequently in equilibrium. The concentration of $\mathrm{HCl}$ in the solutions is different.

Now we shall transfer the mixture of these two solutions into a U-tube, in which they will separate with a sharply defined boundary between them. If we now dip some metal electrodes into the upper ends of the solutions and connect them by a metal conductor, we shall obtain an arrangement of a galvanic chain (fig. 24). We shall choose for our electrodes hydrogen-platinum electrodes which, in contact with a solution containing $\mathrm{H}$-ion, have a well defined potential cal-

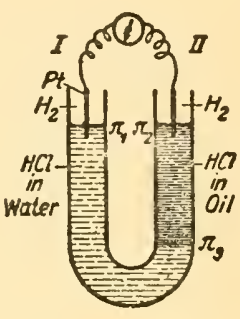

FIG. 24 culable from Nernst's equation. No electric current can originate in this chain, since it is a system in a state of complete equilibrium. Any current should bring about a change in the concentration of the electrolyte, but since we have here a state of equilibrium, no spontaneously occurring process can be produced which would result in a disturbance of the equilibrium. This is an important part of the second law of thermodynamics. Consequently the E.M.F. of this chain must be equal to zero.

On the other hand, we can also estimate the potentials at the electrodes. At electrode I the potential is

$$
\pi_{1}=\mathrm{RT} \ln \frac{\mathrm{c}_{1}}{\mathrm{k}_{1}}
$$

${ }^{3}$ Cf. L. Michaelis, Dynamik der Oberflächen. Dresden 1909.

${ }^{4}$ R. Beutner, Die Entstehung elektrischer Ströme in lebenden Geweben. Stuttgart 1920 . 
where $c_{1}$ is the concentration of hydrogen ions in the water, and $k_{1}$ is that concentration of $\mathrm{H}$-ions in the same solvent against which the potential would be zero. At electrode II the potential is

$$
\pi_{2}=\mathrm{RT} \ln \frac{\mathrm{c}_{2}}{\mathrm{k}_{2}}
$$

with the corresponding meaning for $\mathrm{c}_{2}$ and $\mathrm{k}_{2}$.

The difference between these two electrode potentials is, therefore,

$$
\pi_{1}-\pi_{2}=\operatorname{RT}\left(\ln \frac{\mathrm{c}_{1}}{\mathrm{c}_{2}}+\ln \frac{\mathrm{k}_{2}}{\mathrm{k}_{1}}\right)
$$

This potential difference can become equal to zero when

$$
\frac{c_{1}}{c_{2}}=\frac{k_{1}}{k_{2}}
$$

But since $\frac{\mathrm{k}_{1}}{\mathrm{k}_{2}}$ represents a constant value, therefore, the ratio $\frac{\mathrm{c}_{1}}{\mathrm{c}_{2}}$ must also be constant, i.e., when any acid is distributed in any concentration between an aqueous and an oil phase with the establishment of an equilibrium, the ratio of the $\mathrm{H}$-ion concentrations in the two phases will remain constant. It can be equally well shown that when chlorine electrodes are taken instead of hydrogen electrodes the ratio of $\mathrm{Cl}$-ion concentrations in the two phases will remain the same whatever chloride in whatever concentration be involved. Briefly stated: it can be shown that any given ionic species must be distributed between an aqueous and an oil phase in accord with its unalterable distribution coefficient. But this is not possible, as can be demonstrated both practically and theoretically. The theoretic proof of this impossibility can be given in the sense of Nernst's theory as follows: If we first assume that every ion-species, as well as any other molecular species, has a definite distribution coefficient, then it should certainly be different for the various ion-species. Therefore, in the case of $\mathrm{HCl}$, the $\mathrm{H}$-ions will have a different distribution coefficient from that of the Cl-ions, and the two phases must have different distributions with respect to $\mathrm{H}$ - and Cl-ions. But for electrostatic reasons this is impossible. Neither can the true distribution correspond to the specific coefficients of the $\mathrm{Cl}$ - or $\mathrm{H}$-ions, but it must lie somewhere in between these. And, likewise, this true distribution must be different in an $\mathrm{HCl}$ solution from 
that in an aniline chloride solution. Hence it is not possible that the distribution of $\mathrm{Cl}$-ions should always be the same regardless of the kind or of the amount of chloride present, and consequently the ratio $\frac{c_{1}}{c_{2}}$ cannot be constant under all conditions.

Practically, this point was demonstrated by R. Beutner as follows: when water and an oil phase, such as guaiacol, were shaken on the one hand with $\mathrm{NaCl}$, and on the other hand with aniline chloride, it was found that the aqueous phase took up more $\mathrm{Cl}$ when $\mathrm{NaCl}$ was used than when aniline chloride was used. Evidently that which applies to $\mathrm{Cl}$ as such need not by all means apply to $\mathrm{Cl}^{-}$-ions, and the same was found when the conductivity of the guaiacol solutions in the two cases was determined. In the case of the aniline chloride the Cl-ion content of the guaiacol was much higher than in the case of $\mathrm{NaCl}$. Hence the distribution of $\mathrm{Cl}$-ions, or, in general of any ion species, between water and guaiacol is not in all cases the same.

Since the ratio $c_{1} / c_{2}$ in equation (I) represents a constant value, and $k_{1} / k_{2}$ being a constant, then $\pi_{1}-\pi_{2}$ must always be other than zero. But since the E.M.F. of the whole chain must be zero, it follows that a third potential difference $\pi_{3}$ must be present, and it must amount to $\pi_{1}-\pi_{2}$. The algebraic sum of these three values must be equal to zero. This potential $\pi_{3}$ resides in the boundary surface of the water and oil phases.

The potential difference at the boundary of two phases which contain an ion in common in the concentrations $c_{1}$ and $c_{2}$ is

$$
\pi=\operatorname{RT}\left(\ln \frac{c_{1}}{c_{2}}+\ln \frac{k_{2}}{k_{1}}\right)
$$

Here $k_{2}$ is that concentration of this ion in phase II against which a metal electrode of the same kind would produce zero potential, and $k_{1}$ is the concentration of the same ion in phase $I$ against which a metal electrode of the same kind would likewise yield zero potential. Or, stated otherwise, $k_{1}$ and $k_{2}$ are the electrolytic solution tensions of the ionic species concerned in phases I and II respectively.

Thus these two values, $k_{2}$ and $k_{1}$, are quite problematical quantities which frequently have no physical meaning. For, if we should wish to use, for example ammonium acetate as the electrolyte, we find that we have available neither an "ammonium-metal" nor" an 
acetate-ion yielding gas to use for our electrodes. And so $k_{2}$ and $\mathrm{k}_{1}$, are in reality nothing but integration constants for purposes of calculation. In fact, $k_{2} / k_{1}$ is but a single constant to which we attribute the same significance. Hence we can state that

$$
\pi=\mathrm{RT} \ln \frac{\mathbf{c}_{1}}{\mathbf{c}_{2}}+\mathrm{K}
$$

And yet this constant $\mathrm{K}$ does possess important meaning, its value relates specifically to the ion-species, being a definite characteristic value of every ion-species.

If a current-yielding chain be arranged to contain two such phase boundaries with a potential at each, then the total potential $\mathrm{E}$ of the system will be

$$
\mathrm{E}=\pi-\pi_{1}=\mathrm{RT} \ln \frac{\mathrm{c}_{1}}{\mathrm{c}_{2}}+\mathrm{K}-\left(\mathrm{RT} \ln \frac{\mathrm{c}^{\prime}{ }_{1}}{\mathrm{c}^{\prime}{ }_{2}}+\mathrm{K}^{\prime}\right)
$$

Only in that case, where all the phases have only one ion-species in common will the individual values of $K$ be identical and disappear and $c_{1}, c_{2}, c_{1}{ }^{\prime}, c_{2}{ }^{\prime}$ will then signify only the concentrations of the common ion.

But if the various phases involved contain more than one ionspecies in common the equation can just as well be applied to each of these ions. But since in all combinations of phases in which water is included H-ions are present, it would follow for all such chains

$$
\pi=\mathrm{RT} \ln \frac{\left[\mathrm{H}^{+}\right]_{1}}{\left[\mathrm{H}^{+}\right]_{2}}+\text { Constant }
$$

where the constant depends upon the nature of the solvent only. This result may be easily deduced from Beutner's general equation (even though Beutner himself did not come to this conclusion). Nevertheless it is still very difficult to determine experimentally the hydrion concentration in non-aqueous phases.

So much for the thermodynamic explanation of phase boundary potentials. It will be worth while to add another method of representation, that of Nernst, which is the older one in point of time. It follows from Nernst's conception of the distribution of the salt in the two solvents. The final state attained corresponds, as was shown above, neither to the true distribution equilibrium of the 
cations nor to that of the anions. If $\mathrm{KCl}$ is used, the oil phase will contain either too much $\mathrm{K}^{+}$and too little $\mathrm{Cl}^{-}$, or perhaps the reverse. There will be a tendency in the system to approach the true equilibrium for each of the ionic species. Since because of this tendency a separation of the ions is required, a difference of potential will develop. And, furthermore, the separation will be accompanied by the formation of an electric double layer of ions whose potential will be such that the separating force will be counterbalanced by the attraction between the two layers of ions.

The calculation of this effect can be carried out in some such manner as the following. ${ }^{5}$ Suppose that equilibrium having been reached the concentrations of the electrolyte in the two solutions are $c_{1}$ and $c_{2}$. Now the cations tend to distribute themselves in the ratio of $1: \alpha$ where $\alpha$ is the true distribution coefficient of the cation in question, and the anions likewise tend to distribute themselves in the ratio of $1: \beta$. This tendency is opposed by the electrostatic forces which develop because of this unequal distribution of ions. When one mol of an ionic species migrates from solution 1 to solution 2 (in order to attain true equilibrium), then an electric current is produced yielding work amounting to $\pi \mathrm{F}$. If the same replacement or transposition of ions occurred mechanically (osmotically) then the amount of work RT $\ln \frac{c_{1} \alpha}{c_{2}}$ would be produced. We can think of this transition as occurring in two stages. First one mol of cations is transferred from one medium in which its concentration is $c_{1}$ into a medium of another kind where the concentration is $c_{1} \alpha$. This process does not require any work. Now we dilute the latter solution from concentration $c_{1} \alpha$ to $c_{2}$ which requires the amount of work RT $\ln \frac{c_{1} \alpha}{c_{2}}$. The actual stable distribution which ensues must be so defined that

$$
\pi=\frac{\mathrm{RT}}{\mathrm{F}} \ln \frac{\mathrm{c}_{1} \alpha}{\mathrm{c}_{2}}
$$

By the same reasoning we arrive at the second condition, namely,

$$
\pi=-\frac{\mathrm{RT}}{\mathrm{F}} \ln \frac{\mathrm{c}_{1} \beta}{\mathrm{c}_{2}}
$$

- As given by Michaelis; cf. footnote 3. 
and hence

$$
\pi=\frac{\mathrm{RT}}{\mathrm{F}} \ln \frac{\mathrm{c}_{1} \cdot \alpha}{\mathrm{c}_{2}}=-\frac{\mathrm{RT}}{\mathrm{F}} \ln \frac{\mathrm{c}_{1} \cdot \beta}{\mathrm{c}_{2}}
$$

Therefore, whenever $\alpha=\beta$, then $\pi=0$.

By determining analytically (by conductivity measurements or similar methods) $c_{1}$ and $c_{2}$, we derive the following two equations defining the three quantities, $\pi, \alpha$ and $\beta$ :

$$
\begin{gathered}
\pi=\frac{\mathrm{RT}}{\mathrm{F}} \ln \frac{\mathrm{c}_{1} \cdot \alpha}{\mathrm{c}_{2}} \\
\frac{\mathrm{c}_{1} \cdot \alpha}{\mathrm{c}_{2}}=\frac{\mathrm{c}_{2}}{\mathrm{c}_{1} \beta} \text { or } \frac{\mathrm{c}_{2}}{\mathrm{c}_{1}}=\sqrt{\alpha \cdot \beta}
\end{gathered}
$$

If we compare the former equation

$$
\pi=\frac{\mathrm{RT}}{\mathrm{F}} \ln \frac{\mathrm{c}_{1}}{\mathrm{c}_{2}}+\frac{\mathrm{RT}}{\mathrm{F}} \ln \frac{\mathrm{k}_{2}}{\mathrm{k}_{1}}
$$

with the present equation which we will now write in the form of

$$
\pi=\frac{\mathrm{RT}}{\mathrm{F}} \ln \frac{\mathrm{c}_{1}}{\mathrm{c}_{2}}+\frac{\mathrm{RT}}{\mathrm{F}} \ln \alpha
$$

we see at once that

$$
\frac{\mathrm{k}_{2}}{\mathrm{k}_{1}}=\alpha
$$

and that likewise for the second ion-species it is

$$
\frac{\mathrm{k}_{2}}{\mathrm{k}_{1}}=\beta
$$

Thus we see that the distribution coefficient of an ion-species bears a definite, and a thermodynamically calculable, relationship to the above defined values $k_{2}$ and $k_{1}$.

This principle can be restated as follows:

The oil-solubility (in terms relative to water-solubility) of an ionspecies bears a thermodynamically deducible relationship to its electrolytic solution tension in the oil. A truly amazing conclusion!

The relation of solubility to electrolytic solution tension can also be derived in the following way: 
Let us take a special case in which the two ion species are distributed between the phases I and II according to their true distribution coefficients. (This is only possible when both the anion and cation of the electrolyte used have the same distribution coefficient $\alpha)$. Then each of the ions is in concentration $\alpha \mathrm{c}$ in phase $\mathrm{I}$ and in concentration $\mathrm{c}$ in phase II. Now, if we dip reversible metal electrodes into the two solutions, we obtain as the electrode potential on the left, $\pi_{1}=\mathrm{RT} \ln \frac{\mathrm{c} \alpha}{\mathrm{A}}$, and on the right $\pi_{2}=\mathrm{RT} \ln \frac{\mathrm{c}}{\mathrm{B}}$, where $A$ and $B$ denote the electrolytic solution tensions on the left and on the right side respectively. Since in this case a boundary potential cannot develop, and hence the chain cannot yield a current, it follows that $\pi_{1}-\pi_{2}=0$, i.e.:

$$
R T \ln \frac{\mathrm{c} \alpha}{\mathrm{A}}=\mathrm{RT} \ln \frac{\mathrm{c}}{\mathrm{B}}
$$

or

$$
\begin{aligned}
\frac{\alpha}{\mathrm{A}} & =\frac{1}{\mathrm{~B}} \\
\alpha & =\frac{\mathrm{A}}{\mathrm{B}}
\end{aligned}
$$

Stated in words this means: Every ion-species tends to so distribute itself between two phases that the ratio of its concentrations (or better, its active masses) in these phases should be the same as that of the electrolytic solution tensions of similar electrodes in the same phases. The attainment of such an equilibrium, as was said above, is only then possible, when the distribution coefficients of the anion and cation are equal. In general, however, this kind of distribution is prevented by the resulting potential difference. Then from the joint action of the two partial forces, the tendency towards distribution and the electrostatic force, a kind of equilibrium results, which is different from the one to be expected on the basis of the true distribution coefficients.

\section{Phase boundary chains}

The same happens with a single phase potential as with a single electrode potential: it does not generate any electric current. Only a combination of two different metal electrode potentials or of two 
different boundary potentials produces under certain conditions (not always) a current-yielding chain.

One such arrangement would be:

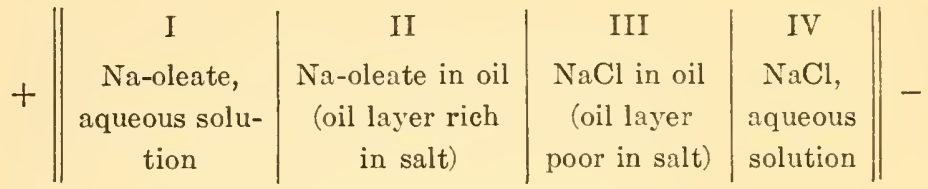

$$
\begin{aligned}
& 12 \\
& \pi_{1} \pi_{2}
\end{aligned}
$$

The lack of equilibrium between two contiguous layers will be found in this system at 2. But this is not a phase boundary and it can only be the seat of an ordinary diffusion potential. As was stated above (page 176), such a potential for the common salts may amount at most to a few millivolts, while this chain produces several centivolts. We have here two phase boundaries, both behaving as metal electrodes; and the difference between their potentials constitutes the E.M.F. of this metal-free chain, which could be closed by means of a saturated $\mathrm{KCl}$ solution and thus rendered free of diffusion potentials as well as metal-free.

In order to obtain large E.M.F. values it is important to choose an inorganic salt and an organic salt both of which are easily soluble in the oil phase. This is comparable to a metal-containing chain in which metals as far apart as possible in the electromotive series are chosen as electrodes.

The calculation of the E.M.F. of a chain of this type is begun by finding that ion-species which is common to all the phases. In this case it is the Na-ion. This chain can be designated as "a reversible chain with respect to $\mathrm{Na}^{+} . "$ If now we designate the concentrations of $\mathrm{Na}^{+}$in the four vessels I, II, III and IV of the chain as $\mathrm{c}_{\mathrm{I}}, \mathrm{c}_{\mathrm{II}}, \mathrm{c}_{\mathrm{III}}$ and $\mathrm{c}_{\mathrm{IV}}$ respectively, we shall have at boundary surface 1 :

$$
\pi_{1}=\mathrm{RT} \ln \frac{\mathrm{c}_{\mathrm{I}}}{\mathrm{c}_{\mathrm{II}}}+\mathrm{K}
$$

and at the boundary 3 :

$$
\pi_{2}=\mathrm{RT} \ln \frac{\mathrm{c}_{\mathrm{IV}}}{\mathrm{c}_{\mathrm{IlI}}}+\mathrm{K}
$$


hence the total E.M.F. of the chain is

$$
\mathrm{E}=\pi_{1}-\pi_{2}=\mathrm{RT} \cdot \ln \frac{\mathrm{c}_{\mathrm{I}}}{\mathrm{c}_{\mathrm{II}}} \cdot \frac{\mathrm{c}_{\mathrm{III}}}{\mathrm{c}_{\mathrm{IV}}}=\mathrm{RT} \ln \frac{\mathrm{c}_{\mathrm{I}}}{\mathrm{c}_{\mathrm{IV}}} \cdot \frac{\mathrm{c}_{\mathrm{III}}}{\mathrm{c}_{\mathrm{II}}}
$$

The potential at the zone of contact 2 is of the character of a diffusion potential, and in most cases may be disregarded as being negligible, especially if it is remembered that the great mobility of the $\mathrm{H}$ - and $\mathrm{OH}$-ions, which in aqueous solutions is the important source of all diffusion potentials, is non-existent in oily solvents.

R. Beutner (l. c.) verified this equation experimentally in the following way:

Guaiacol, whose specific conductance is 0.1 reciprocal megohms (r.m.), is shaken up with $0.1 M$ aqueous solution of $\mathrm{NaCl}$. After the distribution equilibrium is established the conductivity rises to $1.2 \mathrm{r.m}$., an increase of $1.1 \mathrm{r} . \mathrm{m}$. Then another portion of the guaiacol is shaken up with a $0.1 M$ aqueous solution of dimethylaniline hydrochloride. The conductivity rises to 59.0 r.m., an increase of 58.9 r.m. Since the conductances of the various ionspecies are not very different, we may take the ionic concentration as being proportional to the conductance. The conductance of the pure guaiacol must be, of course, subtracted.

Then the E.M.F. of this chain was determined:

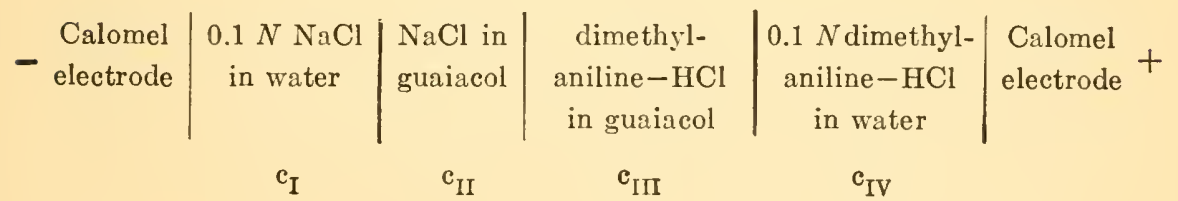

The common ion is $\mathrm{Cl}^{-}$, and we obtain

$$
\frac{\mathrm{c}_{I}}{\mathrm{c}_{\mathrm{IV}}}=1 ; \frac{\mathrm{c}_{\mathrm{III}}}{\mathrm{c}_{\mathrm{II}}}=\frac{58.9}{1.1}
$$

hence

$$
\mathrm{E}=0.0001983 \cdot \mathrm{T} \cdot \log \frac{58.9}{1.1}=0.100 \mathrm{volt}
$$

The observed value was 0.091 volts.

The figures in table 28 were obtained in a similar way for guaiacol 
chains, in which other electrolytes instead of dimethylaniline$\mathrm{HCl}$ were employed (the $\mathrm{NaCl}$ side being left unchanged).

If it is kept in mind that the conductance is but an approximate measure of the ionic concentration, it becomes evident that the calculated and observed values are in very significant agreement.

Table 29 shows the results of a few more of Beutner's experiments with $p$-cresol. These relationships represent perhaps some of the most important recent advances in physiological physics, for here we have succeeded for the first time in obtaining electromotive

TABLE 28

\begin{tabular}{|c|c|c|c|}
\hline & \multirow{2}{*}{$\begin{array}{l}\text { Conductance } \\
\text { of guaiacol } \\
\text { after shaking } \\
\text { r.m. }\end{array}$} & \multicolumn{2}{|c|}{ E.M.F. in volte } \\
\hline & & Calculated & Found \\
\hline $0.1 M$ Dimethylaniline $\mathrm{HCl} \ldots . . \ldots \ldots$ & 59.0 & -0.100 & -0.091 \\
\hline $0.1 M$ Aniline $\mathrm{HCl} . \ldots \ldots \ldots \ldots \ldots$ & 10.3 & -0.056 & -0.059 \\
\hline $0.1 M \mathrm{KCl} . \ldots \ldots \ldots \ldots \ldots$ & 1.9 & -0.012 & -0.011 \\
\hline $0.1 M \mathrm{MgCl}_{2} \ldots \ldots \ldots \ldots \ldots$ & 0.3 & +0.043 & +0.045 \\
\hline
\end{tabular}

TABLE 29

\begin{tabular}{|c|c|c|c|c|}
\hline \multirow{2}{*}{$\begin{array}{l}\text { After shaking with } \\
\mathrm{KCl} \ldots \ldots \ldots \ldots \ldots \ldots\end{array}$} & \multirow{2}{*}{$\begin{array}{c}\begin{array}{c}\text { Conduct- } \\
\text { ance of the } \\
\text { oil phase }\end{array} \\
8 \text { r.m. }\end{array}$} & \multicolumn{2}{|c|}{$\begin{array}{l}\text { Calculated E.M.F. (in rolts) } \\
\text { for the chain: }\end{array}$} & \multirow{2}{*}{$\frac{\begin{array}{c}\text { Observed } \\
\text { E.Mi.F. }\end{array}}{0.009}$} \\
\hline & & $-\mathrm{KCl} / \mathrm{KNO}_{3}+$ & 0.013 & \\
\hline $\mathrm{KNO}_{3} \ldots \ldots \ldots \ldots \ldots$ & 13.2 & $-\mathrm{KCl} / \mathrm{KSCN}+$ & 0.022 & 0.025 \\
\hline KSCN... & 19.3 & $-\mathrm{KCl} / \mathrm{NaCl}+$ & 0.005 & 0.009 \\
\hline $\mathrm{NaCl} . . . \ldots \ldots$ & 6.7 & - NaCl/Na-Salic. + & 0.063 & 0.081 \\
\hline Na-salicylate..... & 80 & $-\mathrm{NaCl} / \mathrm{Na}$-Oleate + & 0.117 & 0.120 \\
\hline 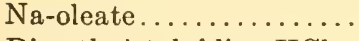 & 630 & & & \\
\hline Dimethyl toluidine- $\mathrm{HCl}$. & 1300 & $+\mathrm{KCl} / \mathrm{Dim}-\mathrm{Tol} \cdot \mathrm{ClH}$ & -0.128 & -0.119 \\
\hline
\end{tabular}

forces of the order of magnitude of those observed physiologically, without the participation of free metals.

It is also possible to arrange chains with phases which have no ion in common, as, for example, the following:

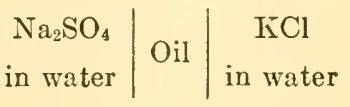

The oil contains $\mathrm{Na}_{2} \mathrm{SO}_{4}$ in its left boundary layer in the corresponding distribution equilibrium, and $\mathrm{KCl}$, in a similar way, in 
its right boundary layer. The E.M.F. of such a chain can be calculated more easily by imagining an aqueous $\mathrm{NaCl}$ solution interposed in the following manner:

$$
\begin{array}{c|c|c|c}
\mathrm{Na}_{2} \mathrm{SO}_{4} \\
\text { in water }
\end{array} \mid \text { Oil }\left|\begin{array}{c}
\mathrm{NaCl} \\
\text { in water }
\end{array}\right| \text { Oil } \mid \begin{gathered}
\mathrm{KCl} \\
\text { in water }
\end{gathered}
$$

By the method outlined above we can calculate the values $\left(\pi_{1}-\pi_{2}\right)$ and $\left(\pi_{3}-\pi_{4}\right)$. Since the total E.M.F. is not affected by the interposition of the $\mathrm{NaCl}$ solution, it follows that $\pi_{2}=\pi_{3}$. Hence the total E.M.F. is

$$
\mathrm{E}=\left(\pi_{1}-\pi_{2}\right)+\left(\pi_{3}-\pi_{4}\right)
$$

where each of the terms is calculable.

\section{The direction of the current in phase boundary chains}

In Beutner's chains, in which an oil phase is the middle member, the direction of the current may vary. It depends upon whether cation or anion is considered the common ion species. Thus in the arrangement:

$$
\begin{array}{c|c}
\text { Water } \\
+\mathrm{NaCl}
\end{array} \mid+ \text { salicylic acid } \mid \begin{gathered}
\text { Water } \\
+\mathrm{NaCl}
\end{gathered} \quad\left(\mathrm{c}_{1}>\mathrm{c}_{2}\right)
$$

the electrolytes distribute themselves in the vicinity of the boundary surfaces, as soon as the phases are brought into contact. A trace of $\mathrm{NaCl}$ passes from the water into the oil, and a small amount of salicylic acid passes from the oil into the aqueous phase. In the oil phase, the mixture $\mathrm{NaCl}+$ salicylic acid is partly changed into Na-salicylate $+\mathrm{HCl}$. In the aqueous phase this rearrangement does not occur, for here the weaker salicylic acid does not displace the much stronger hydrochloric acid from its salts. In the oil, on the other hand, as it was pointed out on page 151, the difference between weak and strong acids is not nearly so pronounced, and the above change may and does occur. Hence, as a result of electrolytic dissociation, we find in the oil phase the following ions: Salicylate- $\mathrm{Na}^{+}$, and traces of $\mathrm{H}^{+}$. The composition of the oil phase is not homogeneous, for each of its boundary surfaces attains a 
state of equilibrium with respect to its adjacent aqueous phase. Actually, the ionic distribution may be represented as:

\begin{tabular}{|c|c|c|}
\hline Water & OiI & Water \\
\hline $\mathrm{Na}^{+}\left(\mathrm{c}_{1}\right)$ & Salicylate $^{-}$ & $\mathrm{Na}^{+}\left(\mathrm{c}_{2}\right)$ \\
\hline $\mathrm{Cl}^{-}\left(\mathrm{c}_{1}\right)$ & $\mathrm{Na}^{+} \mathrm{Na}^{+}$ & $\mathrm{Cl}^{-}\left(\mathrm{c}_{2}\right)$ \\
\hline $\left.\begin{array}{l}\text { Salicylate } \\
\mathrm{H}^{+}\end{array}\right\}$in traces & $\left(\begin{array}{ll}c_{1}^{\prime} & \left(c^{\prime}{ }_{2}\right)\end{array}\right.$ & $\left.\begin{array}{l}\text { Salicylate } \\
\mathrm{H}^{+}\end{array}\right\}$in traces \\
\hline
\end{tabular}

If we neglect the ions present in traces only the arrangement becomes:

\begin{tabular}{|c|c|c|}
\hline Water & Oil & Water \\
\hline $\mathrm{Na}^{+}\left(\mathrm{c}_{1}\right)$ & Salicylate $^{-}$ & $\mathrm{Na}^{+}\left(\mathrm{c}_{2}\right)$ \\
\hline $\mathrm{Cl}^{-}\left(\mathrm{c}_{1}\right)$ & $\begin{array}{ll}\mathrm{Na}^{+} & \mathrm{Na}^{+} \\
\left(\mathbf{c}_{1}^{\prime}\right) & \left(\mathrm{c}^{\prime}{ }_{2}\right)\end{array}$ & $\mathrm{Cl}^{-} \quad\left(\mathbf{c}_{2}\right)$ \\
\hline
\end{tabular}

Hence, the ion common to all three phases is the Na-ion only.

We can, therefore, trace the formation of the potential differences at the phase boundaries to the tendency of Na-ions present in the oil, in a state of electrostatically evoked equilibrium, to approach the true equilibrium, such as would ensue in the absence of electrostatic opposition, by repassage into the aqueous phase. The free Na-ions upon reaching the water phase render it positive with respect to the oil, and that side of the water phase is the more positive which is adjacent to the part of the oil phase containing the higher concentration of Na-ions. If, however, the common ion is a negative ion, as in the chain:

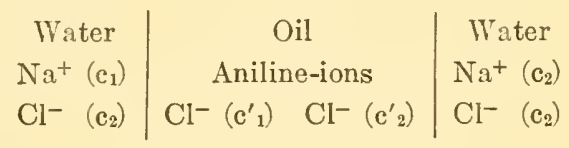

then the same considerations apply to the Cl-ion, and the side which was the more positive in the formel chain becomes in this chain the more negative. This is the manner in which the direction of the current is determined in these chains.

\section{The concentration effect}

When two aqueous solutions containing an electrolyte in different concentrations, $c_{1}$ and $c_{2}$, are separated by an oil phase, then, according to our experience, the E.M.F., of such a chain may lie 
within two definite limiting values. The one limiting value is that E.M.F. which would be yielded by an ordinary concentration chain containing a metal for the common ion species involved, or $=\mathrm{RT} \ln \frac{\mathrm{c}_{1}}{\mathrm{c}_{2}}$, with the sign depending on the positive or negative charge of the ion. The other limiting value of the E.M.F. is zero. Between these two fall all the observed values.

The necessary condition for the maximal concentration effect is that the concentration of the common ion-species in the entire mass of the oil phase should be constant, although the concentration of this common ion in the two adjacent aqueous phases is different. For when in the equation given on page 193

$$
\mathrm{E}=\mathrm{RT} \ln \frac{\mathrm{c}_{\mathrm{I}}}{\mathrm{c}_{\mathrm{IV}}} \times \frac{\mathrm{c}_{\mathrm{III}}}{\mathrm{c}_{\mathrm{II}}}
$$

$c_{\mathrm{Ir}}$ becomes $=\mathrm{c}_{\mathrm{IV}}$, and we actually obtain

$$
\mathrm{E}=\mathrm{RT} \ln \frac{\mathrm{c}_{1}}{\mathrm{c}_{2}}
$$

If this condition is incompletely or not at all fulfilled, as when the concentration of the common ion is weaker on the boundary of the oil phase with the weaker aqueous solution than it is on the boundary of the oil with the stronger solution, or when, in other words, $\mathrm{c}_{\mathrm{I}}<\mathrm{c}_{\mathrm{IV}}$ and $\mathrm{c}_{\mathrm{II}}<\mathrm{c}_{\mathrm{III}}$, then $\mathrm{E}<\mathrm{RT}$ 1n $\frac{\mathrm{c}_{1}}{\mathrm{c}_{2}}$.

Let us see just when this condition for the maximal concentration effect is fulfilled.

1. The first example of the maximal concentration effect is the so-called glass-chain, which has been first used by Cremer. ${ }^{6}$ This author was the first to conceive the idea of imitating a physiological membrane by means of an extremely thin walled glass sheath, which can be easily blown out at the end of a test tube. In this way glass bulbs are obtained whose walls are a few hundredths of a millimeter in thickness, and which show at room temperatures, or better at $40-50^{\circ}$, a distinctly demonstrable electric conductivity. In accord with already established ideas concerning the conductivity of glass, Cremer had to assume that he was dealing with true elec-

${ }^{6}$ MI. Cremer, Zeitschr. f. Biol. 47, 1 (1906). 
trolytic conductivity in which ionic transport was involved. For E. Warburg ${ }^{7}$ had already shown that at $200^{\circ} \mathrm{C}$ the conductivity of glass was of electrolytic nature, and undoubtedly Cremer was justified in interpreting the conductivity of much thinner glass walls as being of electrolytic nature. Cremer made the striking observation, which was not appreciated until much later, that an aqueous acid solution inside of his thin glass bulb showed a potential difference of about one volt towards an alkaline solution outside of the bulb (the value observed was 0.55 volts, to which a correction for the diffusion potential had to be added). Cremer recognized the glass wall as the source of this electromotive force, and he also quite correctly recognized the great significance of the acidity of the solution in determining the value

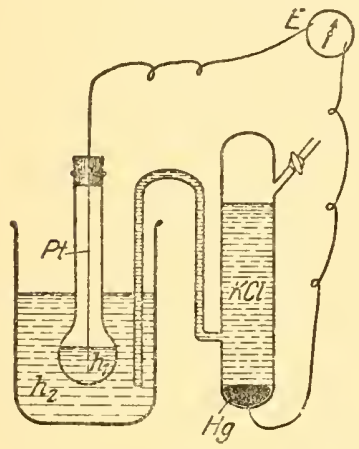

FIG. 25. Glass-chain of this E.M.F. But his interpretation is somewhat different from that given later by Haber.

Haber and Klemensiewicz ${ }^{8}$ were the first to advance the theory which is still held today, and they made exact measurements and extensive application of the glass chain. When two solutions of unequal hydrion concentration are separated by an extremely thin glass wall, a potential difference arises between the two solutions which is of the same magnitude as that developed in a hydrogen gas chain as described on page 161 . The arrangement of the glass chain is as shown in figure 25.

The solution of hydrion concentration $h_{2}$ is placed in a large beaker the solution of hydrion concentration $h_{1}$ is in a very thin walled glass bulb which is immersed into the first solution. The current is led away on one side by means of a calomel electrode and on the other by a platinum wire (instead of the latter it would be better to use a calomel electrode also, in order that the two electrode potentials compensate each other). $\mathrm{E}$ is an electrometer, best of all a binant electrometer. If in a series of experiments the inner solution be kept constant and the outer solution varied, we find for a

${ }^{7}$ Cited by Georg Meyer, Wiedemanns Ann. 40, 244 (1890).

${ }^{8}$ F. Haber and Klemensiewicz, Ann. d. Physik [4] 26, 927 (1908) 
tenfold change in $h_{2}$ a difference of 58 millivolts. All other ionic species which may be present in the solutions are without effect, as was recently stated by Freundlich and Rona. ${ }^{9}$

The glass chain may be used as well as the gas chain for $\mathrm{pH}$ measurements, and especially for electrometric titration for which Haber gives beautiful examples. The glass chain represents a chain reversible for $\mathrm{H}$-ions only, in distinction from Beutner's oil-chains which, as we saw above, can be arranged to be reversible for any given anion or cation. The only possible explanation, in Haber's sense, is that the $\mathrm{H}$-ion constitutes the common ion for the two aqueous phases and for the glass phase, and that the concentration of $\mathrm{H}$-ions in the glass is constant and is independent of the adjacent

TABLE 30

\begin{tabular}{|c|c|c|c|}
\hline \multirow{2}{*}{$c_{l}$} & \multirow{2}{*}{$\mathbf{c}_{2}$} & \multicolumn{2}{|c|}{ E.M.F. } \\
\hline & & Observed & $\begin{array}{l}\text { Calculated maximal } \\
\text { effect* }\end{array}$ \\
\hline$M$ & $M$ & volts & volts \\
\hline$\frac{1}{2}$ & $\frac{1}{10}$ & 0.021 & 0.040 \\
\hline$\frac{3}{10}$ & $\frac{1}{50}$ & 0.025 & 0.040 \\
\hline $5^{1} 0$ & 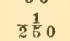 & 0.034 & 0.040 \\
\hline $2 \frac{1}{5} 0$ & $\frac{1}{1250}$ & 0.041 & 0.040 \\
\hline
\end{tabular}

${ }^{*} c_{1}: c_{2}$ ratio in all of these cases is $1: 5$. Hence the calculated maximal effect is $\pi=0.058 \times \log 5=0.0406$ volts.

media. Haber assumes that the glass contains traces of water of imbibition (swelling), and that this factor determines the $\mathrm{H}$-ions in the glass.

2. As we saw above, Beutner succeeded in obtaining more or less complete concentration effects by employing oily liquids as the middle phase. The first used by him were chains with salicylic aldehyde as the oil phase:

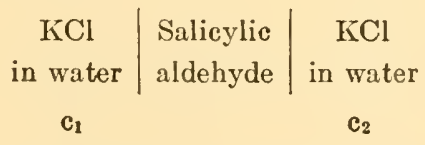

with these he found the E.M.F. values given in table 30 .

${ }^{9} \mathrm{H}$. Freundlich and P. Rona, Sitzungsber. d. Preuss. Akad. d. Wissensch. (1920), 397. 
It is a striking result that at the lower salt concentrations the effect obtained approaches the calculated maximal effect, while at the higher concentrations the observed values are increasingly low. The obtained values were temporarily completely reversible; and in a series of experiments when $c_{1}$ was kept constant while $c_{2}$ was varied in both directions, Beutner always obtained the same E.M.F. values for the same values of $c_{2}$.

As the necessary condition for the maximal effect we specified above that the concentration of the common ion should be the same at the two sicles of the oil phase, while it is unequal in the two aqueous phases. Let us now see how this condition is fulfilled in this particular chain. Salicylic aldehyde is decidedly acid in character, partly because of its phenolic $\mathrm{OH}$-group and partly because it always contains traces of salicylic acid. In contact with water almost none of these acid groups pass into the aqueous phase, since the distribution coefficient with respect to water is here extremely small. But a small amount of $\mathrm{KCl}$ passes from the contiguous aqueous phase into the oil phase. But, as we saw in the chapter on dissociation in non-aqueous solvents, hydrochloric acid in oil does not behave as a strong but as a weak acid, and hence we have in the oil phase the double decomposition:

$\mathrm{KCl}+$ salicylic acid $\rightleftarrows \mathrm{K}$-salicylate $+\mathrm{HCl}$

a reaction which could not have occurred in aqueous solution. In this way the $\mathrm{KCl}$ equilibrium is disturbed, the salicylic acid attracting, to a certain extent, more K-ions into its solution. If in the oil phase salicylic acid should be a stronger acid than $\mathrm{HCl}$, then the former will not remain in the free state, but it will attract enough potassium to transform all of the acid in the oil into the $\mathrm{K}$-salt, independently of the $\mathrm{KCl}$ concentration in the aqueous phase. In this case, the $\mathrm{K}^{+}$-concentration will be the same throughout the oil phase. But should the salicylic acid in the oil solution not behave as a stronger acid than $\mathrm{HCl}$, but rather dissociate to the same extent as $\mathrm{HCl}$, even then the same result will ensue, with greater or lesser completeness, depending upon the conditions.

When the $\mathrm{KCl}$ concentration in the water phase is very small, then, after the establishment of equilibrium, the $\mathrm{K}^{+}$will be present in the oil almost entirely as $\mathrm{K}$-salicylate. But when the $\mathrm{KCl}$ concentration in the water is very great, then it will also be greater in 
the oil, and the potassium will be present in the oil not only as the salicylate but also, to a relatively marked degree, as the chloride. In the latter case the total amount of $\mathrm{K}^{+}$in the oil phase can no longer remain independent of the $\mathrm{HCl}$ concentration in the water phase. This is why it happens that the concentration effect approaches its maximal value the more closely the more dilute the aqueous $\mathrm{KCl}$ solutions, which was experimentally confirmed, as shown above.

When $\mathrm{NaCl}$ was used instead of $\mathrm{KCl}$ the E.M.F. values shown in table 31 were found.

The concentration effect is smaller in this case, but the effect of the absolute salt concentration is still quite obvious.

TABLE 31

\begin{tabular}{c|c|c|c}
\hline & & \multicolumn{2}{|c}{ E.M.F. in volts } \\
\cline { 2 - 3 }$c_{1}$ & $c_{2}$ & Observed & Calculated \\
\hline$\frac{1}{10}$ & $5^{1}$ & 0.024 & 0.040 \\
$5^{1} 0$ & $\frac{1}{2}$ & 0.026 & 0.040 \\
$\frac{1}{2} \frac{1}{5} 0$ & $1 \frac{1}{25} 0$ & 0.030 & 0.040 \\
\hline
\end{tabular}

Another oil-chain in which the E.M.F. values closely approached the maximal effect was the following:

\begin{tabular}{c|c|c} 
Water & Salicylic & Water \\
dimethylaniline $-\mathrm{HCl}$ & aldehyde & dimethylaniline $-\mathrm{HCl}$
\end{tabular}

$\mathrm{c}_{1}$

$\mathrm{c}_{2}$

$\left(c_{1}>c_{2}\right)$

\begin{tabular}{c|c|c|c}
\hline & & \multicolumn{2}{|c}{ E.M.F. } \\
\hline molar & $c_{2}$ & Observed & Calculated \\
\hline 0.1 & molar & volts & volts \\
0.02 & 0.02 & 0.042 & 0.040 \\
& 0.004 & 0.037 & 0.040 \\
\hline
\end{tabular}

In this case also the acidic nature of the oily solvent is responsible for the constant concentration of cations (i.e., dimethylanilineions) within it, in spite of the differences between $c_{1}$ and $c_{2}$. 
On the other hand in the chain

\begin{tabular}{c|c|c} 
Water & $\begin{array}{c}\text { Salicylic } \\
\text { Na-salicylate }\end{array}$ & Water \\
$c_{1}=0.1$ molar & & Na-salicylate \\
$c_{2}=0.5$ molar
\end{tabular}

the observed E.M.F. value was only 0.005 volts, instead of the calculated maximal value of 0.040 volts; thus we have here an approach to the other limiting value of R.M.F. $=0$. This is due to the fact that, in this case, the anion, the salicylate-ion, is so much more soluble in the oil phase than the cation, that it predominates in the distribution. But being an anion it cannot be fixed, as a cation would be, by the acidic oil. Hence the necessary conditions for the maximal effect are lacking; on the side where the aqueous phase contains more salicylate-ions, the oil also contains more of them; or, the concentration of salicylate-ions in the oil phase is not invariable.

The theoretical considerations which determine whether and how far the E.M.F. approaches this or that limit may be stated as follows: The salt tends to distribute itself between the phases. If this distribution is affected by the simultaneously occurring separation of oppositely charged ions, then the difference of potential arises. But if no or equal electrostatic forces interfere on both sides of the oil phase, then no E.M.F. is developed.

The last example, with Na-salicylate, presents the simplest case. If the above theory is correct, then it should be experimentally demonstrable that in this case the common ion can distribute itself according to a definite distribution coefficient and unhindered by other forces. In other words, it must be shown that in those cases where the E.M.F. = 0 the common distribution law for electrolyte is valid and that the ratio of its concentrations in the two phases is invariably independent of the absolute amount present.

This demonstration was given by Beutner for the chain arranged with salicylic aldehyde and sodium salicylate: $12.5 \mathrm{cc}$. of salicylic aldehyde were shaken with $50 \mathrm{cc}$. of a 0.1 molar aqueous solution of sodium salicylate until the distribution equilibrium was established. The conductance of the oil phase which was originally 0.7 r.m. is now 2.56 r.m. On using a 0.5 molar solution of sodium salicylate instead of the 0.1 molar, the conductance is $14.46 \mathrm{r.m}$. These two observed conductances are in the ratio of $1: 5.6$, while the ratio of the concentrations of the two aqueous solutions is $1: 5$. 
Since the conductances are here closely proportional to the concentrations, it is hence demonstrated that distribution law is applicable to the partition of sodium salicylate between an oil and an aqueous phase. Consequently no concentration effect can arise.

On the other hand, when dimethylaniline- $\mathrm{HCl}$ was used in such a chain the relations were as follows:

Salicylic aldehyde shaken with 1 molar aqueous solution of dimethylaniline-HCl................. 7.8 r.m.

Salicylic aldehyde shaken with $1 / 50$ molar aqueous solution of dimethylaniline-HCl.............. 19.9 r.m.

In this case, while the aqueous concentrations were in the ratio of $1: 50$, the ratio of the ionic concentrations in the oil phase was $1: 2.5$, i.e., the distribution law did not apply here. The ionic content of the oil phase remained constant and unaffected by the concentration in the external aqueous phase.

The same conditions are true for Haber's glass chain. Here the common ion is the H-ion, whose presence in the glass is probably due to the dissociation of the traces of contained water. But since only the pure water, and not the dissolved electrolytes, penetrates into the glass, the $\mathrm{H}^{+}$-concentration of the glass remains constant and unaffected by that of the aqueous phase.

The case of the usual concentration chain may also be interpreted in the same sense. Being given two phases, viz., metallic silver and an aqueous solution of $\mathrm{AgNO}_{3}$, it may be said that the silver contains freely motile electrons (since it possesses the metallic property of conductivity), and hence it may be said to contain "Ag-ions." The metal represents, as it were, a phase consisting of a silver salt, whose cation is $\mathrm{Ag}^{+}$and whose anion is its free electron. The aqueous solution, on the other hand, contains only Ag- and $\mathrm{NO}_{3}$-ions, the electrons do not penetrate into the water phase, they are "not water-soluble." Therefore the concentration of "Agions" in the metal is independent of the $\mathrm{Ag}^{+}$-concentration in the solution and is invariable. Hence a metal must yield a maximal concentration effect, when it is arranged in the order of an oil chain. The E.M.F. of the chain

$$
\begin{array}{c|c|c}
\mathrm{Ag} \mathrm{NO}_{3} & \text { Metallic } & \mathrm{AgNO}_{3} \\
\mathrm{c}_{1} & \mathrm{Ag} & \mathrm{c}_{2}
\end{array}
$$


when its ends are led off by means of two similar metal electrodes (such as mercury-calomel-electrodes in $0.1 \mathrm{~N} \mathrm{KCl}$ ) may be easily calculated. All electrode potentials are removed, with the exception of those on the two sides of the silver plate. These two sides, according to Nernst, have in fact the potential difference of RT $\ln \frac{c_{1}}{c_{2}}$, which means that this chain is in reality one form of arrangement of the usual (Nernst's) concentration chain. It can be imagined that the metal silver strip is split into two plates which are connected by a wire, and one of which is in contact only with the left and the other only with the right solution. The whole of this arrangement represents the usual concentration chain into whose circuit are introduced two calomel electrodes, the potentials at which are mutually abolished. It is hence permissible to neglect these two calomel electrodes, and then the above arrangement becomes a typical concentration chain for Ag-ions. This demonstrates that Nernst's concentration chain may be conceived of as a special case of the phase boundary chain.

The following is the simplest case in which the concentration effect $=0$. Two aqueous solutions of different concentrations of the same electrolyte are separated by an oil. The oil is chemically completely inert, is neither acid nor basic in character and does not enter into any chemical reaction with the electrolyte. In such a chain the E.M.F. is zero, in spite of the concentration differences, as in the chain.

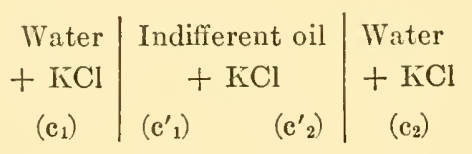

or, in terms of ions,

$$
\begin{array}{l|ll|l}
{\left[\mathrm{K}^{+}\right]=\mathrm{c}_{1}} & {\left[\mathrm{~K}^{+}\right]=\mathrm{c}^{\prime}{ }_{1}} & {\left[\mathrm{~K}^{+}\right]=\mathrm{c}^{\prime}{ }_{2}} & {\left[\mathrm{~K}^{+}\right]=\mathrm{c}_{2}} \\
{\left[\mathrm{Cl} \mathrm{l}^{-}\right]=\mathrm{c}_{1}} & {[\mathrm{Cl}-]=\mathrm{c}^{\prime}{ }_{1}} & {\left[\mathrm{Cl}^{-}\right]=\mathrm{c}^{\prime}{ }_{2}} & {[\mathrm{Cl}-]=\mathrm{c}_{2}}
\end{array}
$$

The essential point involved in this case is that $\mathrm{K}^{+}$concentration is everywhere in the chain equal to the $\mathrm{Cl}^{-}$concentration. In this chain neither $\mathrm{K}^{+}$nor $\mathrm{Cl}^{-}$may be regarded as the common ion. In the case of the $K^{+}$we should have as was shown on page 193 .

$$
\mathrm{E}=\mathrm{RT} \ln \frac{\mathrm{c}_{1}}{\mathrm{c}_{2}} \times \frac{\mathrm{c}_{2}^{\prime}}{\mathrm{c}_{1}^{\prime}{ }_{1}}
$$


In the case of the $\mathrm{Cl}^{-}$- ions we should have, because of the negative charge of the ion:

$$
\mathrm{E}=-\mathrm{RT} \ln \frac{\mathrm{c}_{1}}{\mathrm{c}_{2}} \times \frac{\mathrm{c}_{2}^{\prime}}{\mathrm{e}_{1}^{\prime}}
$$

Both equations must be correct, and this can only be possible when $\mathrm{E}=0$ (or, what amounts to the same thing, when the fraction following $\ln$ is equal to 1 ).

Only whenever it happens that the concentration of the K-ions in any one of the four parts of the chain is different from that of the Cl-ions, can the E.M.F. be other than zero. Such is the case when a second electrolyte is present, or when the oil phase itself is of an acid or basic character.

\section{The ionic series}

The chains considered above contained at both ends solutions of the same electrolyte in different concentrations, and insofar they are analogous to metal concentration chains. But if we employ different electrolytes at the two ends, then we obtain an analogy to chains containing different metals. As the simplest possible case we shall choose that in which two different electrolytes, but possessing an ion in common, are used on the two sides in the same concentration, as, for example,

$$
\begin{array}{l|l|l}
\text { Water } & \mathrm{NaCl}
\end{array} \mid \text { Oil } \mid \begin{aligned}
& \text { Water } \\
& \mathrm{KCl}
\end{aligned}
$$

or,

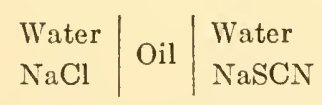

In the first case the common ion is the anion, in the second the cation. Here the E.M.F. of the chains depends upon the difference in the solubility of the two electrolytes in the oil phase. If we assume that the $\mathrm{Cl}^{-}$is more soluble than the $\mathrm{Na}^{+}$ion, then the oil should become negatively charged with respect to the $\mathrm{NaCl}$ solution. But if it should happen that on the other side of the oil the difference between the oil-solubility of $\mathrm{Cl}^{-}$and that of $\mathrm{K}^{+}$is smaller, then the $\mathrm{KCl}$ solution will become less positively charged with respect to the oil. Hence the $\mathrm{KCl}$ solution becomes the negative pole of this chain. Hence, if in a chain of this order, we should al- 
ways use $\mathrm{NaCl}$ at the left end varying the salt at the right end, the latter will be negative whenever the difference between the oilsolubilities of its two ions is less than that between the oil-solubilities of the two ions of the $\mathrm{NaCl}$ solution; and it will be positive when this difference is greater. If we should vary only the cation on the right side solution, the right end will become the more negative the more oil-soluble is the cation in comparison with the $\mathrm{Na}^{+}$ion. If only the anion is varied, then the right end will become the more positive with the increasing oil-solubility of this anion in comparison with the $\mathrm{Cl}^{-}$ion. In Beutner's experiments shown in table $320.1 \mathrm{~N}$ $\mathrm{NaCl}$ solution was used uniformly on the left side (of the oil) and on the right $0.1 \mathrm{~N}$ solutions of the salts indicated in the first column of

TABLE 32

\begin{tabular}{|c|c|c|c|c|}
\hline \multirow[b]{2}{*}{ Salt } & \multicolumn{2}{|c|}{ Acidic oil phase } & \multicolumn{2}{|c|}{ Basic oil phase } \\
\hline & $\begin{array}{c}\text { Salicylalde- } \\
\text { byde } \\
\text { containing } \\
\text { salicylie acid } \\
\text { I }\end{array}$ & $\mid \begin{array}{c}\text { Nitrobenzene } \\
\text { t } 1 \text { MIol } \\
\text { o-Nitroben- } \\
\text { zoic acid } \\
\text { II }\end{array}$ & $\mid \begin{array}{c}\text { Nitrobenzene } \\
+\frac{1}{5} \text {.Iol } \\
\text { Dimethyl- } \\
\text { aniline } \\
\text { III }\end{array}$ & $\begin{array}{c}\text { o-Toluidine } \\
\text { IV }\end{array}$ \\
\hline & millivolts & millivolts & millivolts & millivolts \\
\hline (a) $\mathrm{NaCl}$. & 0 & 0 & 0 & 0 \\
\hline (b) $\mathrm{NaI} . . . .$. & 0 & +3 & +68 & +133 \\
\hline (c) $\mathrm{NaSCN} . . .$. & +15 & +16 & +92 & +140 \\
\hline (d) Dimethylaniline-HCl........ & -110 & -210 & -6 & -25 \\
\hline (e) $\mathrm{KCl} \ldots \ldots \ldots \ldots \ldots \ldots$ & -24 & -44 & 0 & 0 \\
\hline (f) $\mathrm{CaCl}_{2} \ldots \ldots \ldots \ldots \ldots$ & +40 & +2 & -3 & +10 \\
\hline
\end{tabular}

the table. In experiments $\mathrm{a}, \mathrm{b}, \mathrm{c}$ the anion was varied. It will be seen that the positive effect increases in the series $\mathrm{Cl}^{-}, \mathrm{I}^{-}, \mathrm{SCN}^{-}$. We must therefore conclude that this also represents the sequence of the increasing oil-solubility of the ions. In fact, the ionic series remains unaltered regardless of the oil used for the middle phase of the chain. It is true that the quantitative differences between the ions may not remain the same, but the sequence in the series is unchanged These ionic series, which we encounter here for the first time and to which we shall return later more extensively, are as follows:

Decreasing negative effect,-decreasing oil solubility

Cations $\mathrm{K} \quad \mathrm{Na} \quad \mathrm{Ba} \quad \mathrm{Ca} \quad \mathrm{Mg}$

Increasing positive effect,--increasing oil-solubility

$\begin{array}{llllll}\text { Anions } & \mathrm{SO}_{4} & \mathrm{Cl} & \mathrm{Br} & \mathrm{I} & \mathrm{SCN}\end{array}$ 
The nature of the oil in the middle phase is not quite as immaterial as might appear from this tabular summary, for the differences between the individual ions within the series are accentuated to various degrees depending upon the oil. Thus, if the oil is of an acid character (either by itself or in virtue of an added oil-soluble organic acid), then the differences between the cations are strongly marked, while those between the anions are slight. In a basic oil (or with an added organic base) this effect is reversed, as would be expected from the stated theory.

But apart from this general rule specific effects of the nature of the oil manifest themselves. Thus in a chain with $\mathrm{NaCl}$ at one end and $\mathrm{Na}_{2} \mathrm{SO}_{4}$ at the other we should expect from the above rule the $\mathrm{Na}_{2} \mathrm{SO}_{4}$ side to be negative. Such is the case with o-, m-, pcresol, phenol, guaiacol, acetophenone, acetoacetic ester (all of which yield an E.M.F. of 20-30 millivolts); aniline, toluidine (which yield even higher E.M.F. values). On the other hand, benzaldehyde and salicylaldehyde give E.M.F. values of nearly zero, while with cinnamic aldehyde, anisaldehyde and benzophenone even a positive charge of the $\mathrm{Na}_{2} \mathrm{SO}_{4}$ side was found. Evidently the above rules are obeyed by all of those oils which are definitely acid or basic in character in virtue of $\mathrm{NH}_{4}$ or $\mathrm{OH}$-groups; while with oils of a chemically indifferent character the relationships are not so unequivocal, which is quite comprehensible in view of our earlier explanations.

\section{The electromotive series of the oils}

We have hitherto considered chains in which the asymmetry of arrangement lay in the difference between the two aqueous phases. But chains can be had whose aqueous phases are of the same composition and whose oil phase is asymmetrical, the latter consisting of two oils, as, for instance,

$$
\begin{gathered}
\text { I II III } \\
\text { Aqueous phase } \mid \text { Oil I } \mid \text { Oil II | Aqueous phase }
\end{gathered}
$$

Such chains, with the two aqueous phases being identical, yield an E.M.F. obviously caused by the potential difference between the

\begin{tabular}{|c|c|c|c|}
\hline $\begin{array}{c}\text { Calomel electrode } \\
\text { in } 0.1 \mathrm{~N} \mathrm{KCl} \text { in } \\
\text { water }\end{array}$ & Cresol & Benzaldehyde & $\begin{array}{c}\text { Calomel electrode } \\
\text { in } 0.1 \mathrm{~N} \mathrm{ICl} \text { in } \\
\text { water }\end{array}$ \\
\hline
\end{tabular}
single potentials I and III. As for example, in the chain 
Some of Beutner's measurements are shown in table 33.

Thus we have an "electromotive series" for oily liquids as well as for the metals.

But also the chain

$$
\begin{array}{c|c|c|c}
\text { Aqueous } & \text { Nitrobenzene } & \text { Aqueous } \\
\text { salt solution }
\end{array} \mid \text { Nitrobenzene } \mid \begin{gathered}
\text { Nitric acid } \\
+ \text { picric solution }
\end{gathered}
$$

studied long ago by Cremer ${ }^{10}$ may also be included in this group of chains. For in this case the salt distribution on the two sides is unequal; the dissolved picric acid effecting a higher concentration of cations, which, in addition, is but little dependent upon the concentration of the salt in the adjacent aqueous phase.

\section{TABLE 33}

\begin{tabular}{|c|c|}
\hline & VoLTs \\
\hline Benzaldehyde with Cresol............. & -0.13 \\
\hline Benzaldehyde with Phenol............... & +0.13 \\
\hline 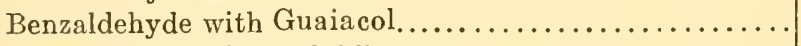 & +0.08 \\
\hline 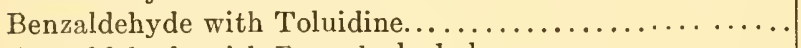 & +0.05 \\
\hline Benzaldehyde with Benzyl alcohol............ & +0.04 \\
\hline 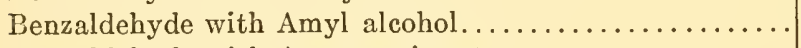 & +0.02 \\
\hline 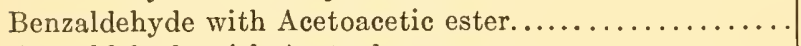 & -0.05 \\
\hline 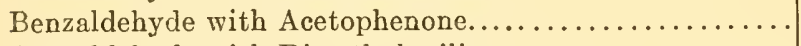 & -0.07 \\
\hline 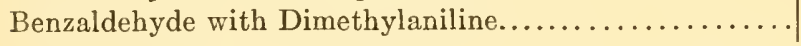 & -0.09 to -0.13 \\
\hline
\end{tabular}

The nitrobenzene dissolved on the right side in the picric acid imparts to the latter a new character. The addition of an organic acid to an oil renders the oil "more positive" in the electromotive series. In pure oils, however, it has not been found as a rule that they are more positive according to the strength of their acidity. Furthermore, it is not merely a question of the amount of acid in the oil, but also of the degree of dissociation of this acid. The dielectric constant of the oil phase is also of great importance. Of two oils containing the same amount of acid the one with the smaller dielectric constant is the more negative, Thus:

Nitrobenzene saturated with nitrobenzoic acid

\begin{tabular}{c|c} 
Without added & $\begin{array}{c}+ \text { an equal } \\
\text { volume of } \\
\text { benzene }\end{array}$
\end{tabular}

${ }_{10}$ M. Cremer, Zeitschr. f. Biol. 47, 1 (1906). 
The benzene, diminishing the diclectric constant of the oil phase decreases the dissociation of the dissolved acid and thus renders the oil phase more negative. ${ }^{11}$ The correlation of the chemical constitution of an oil with its position in the electromotive series still needs further investigation.

\section{Physiological applications of the theory of boundary potentials}

It appears appropriate at this juncture to examine the relation of the above observations to the electromotive forces found in living tissues. In fact, these currents produced without metals, observed by the physiologists several decades ago, served as a stimulus for the above described studies which were undertaken to elucidate biological processes. The fundamental physiological observations date back to DuBois-Reymond ${ }^{12}$ which stated briefly were as follows: Whenever a living tissue (muscle, nerve, leaf or fruit) was injured (cut, crushed, etc.) at any place, and an injured and an uninjured place were connected by means of unpolarizable electrodes an electric current of $0.02-0.1$ volts was produced, the injured spot being negative with respect to the uninjured spot. Also whenever the uninjured surfaces of any two related organs (tendon and muscle, root and leaf or stem) were connected in the same manner, a current was obtained (the tendon being negative towards the muscle, the root negative towards the leaf). These observations provoked the hope of explaining the nature of vital processes on the basis of electric phenomena. Physical science of that day had not yet reached an adequate interpretation of those observations; for it was then still impossible to reproduce so great potential differences without the use of metals. A "molecular theory" attempted by DuBois-Reymond was of a purely descriptive character. Soon afterwards L. Hermann ${ }^{13}$ discovered that injured parts of the same tissue showed no potential differences towards each other, and hence Rule,

11 This finding will be recalled later in the section dealing with Coehn's

${ }^{12}$ E. Du Bois-Reymond, Untersuchungen über tierische Elektrizität. Berlin 1848, 1849, 1860, C. Reimer; Gesammelte Abhandlungen zur Muskel und Nervenphysik. Leipzig 1875; see also Helmholtz, Allgemeine Monatsschr. f. Literatur u. Kunst. Kiel, April 1852.

${ }^{13}$ L. Hermann, Weitere Untersuchungen zur Physiologie der Muskeln und Nerven. Berlin 1867. 
he ascribed the observed current to the destructive (death) processes at the place of injury, a theory which subsequently was expanded into the so-called "alteration theory." When, later, diffusion potentials became better known, it was attempted to utilize them for the explanation of these phenomena. ${ }^{14}$ But diffusion potentials of corresponding order of magnitude can arise only as an exception in the diffusion of free mineral acids toward neutral solutions, and besides, they demand conditions never met with in the living organism. Even when the presence of such acids as lactic acid is taken into account, the maximal diffusion potentials could amount to no more than a few millivolts, whereas currents of injury usually amount to several centivolts. Wilhelm Ostwald ${ }^{15}$ gave a material stimulus to the reinvestigation of this problem. In the course of an investigation of the electrical properties of precipitation membranes he expressed the conjecture that the currents observed in muscles and nerves, and even in the electric fishes, have their origin in the properties of similar membranes. He ascribed to these membranes the peculiarity of being permeable either only to anions or only to cations. The diffusion of an electrolyte through such a membrane would therefore result in a separation of the ions and hence in a potential difference. This explanation has the great distinction of being the first to introduce the phase boundary as the place of orjgin of an electromotive force. And yet the conception of ionic permeability did not prove to be capable of adequate and quantitative investigation. To be sure, the physiologists attempted to extend this theory by trying to find the ions to which living membranes are impermeable. Bernstein ${ }^{16}$ ascribed this property to the K-ions. Höber ${ }^{17}$ could not confirm this. $\mathrm{He}$ thought to have shown that the permeability of the cell membrane to the different ions varied with the effect of different ions upon the membrane. He assumed that the colloidal state of the membrane changed under the influence of the various ions, and that along with it the permeability varied. This assumption appeared all the more plausible, as the ionic series, so frequently encountered

${ }^{14}$ Oker-Blom, Pflügers Arch. f. d. ges. Physiol. 84, 191 (1901).

15 Wi. Ostwald, Zeitschr. f. physikal. Chem. 6, 71 (1890).

${ }^{16}$ Bernstein, Pflügers Arch. f. d. ges. Physiol. 92, 521 (1902).

${ }^{17}$ Höber, Physikalische Chemie der Zelle und Gewebe. Leipzig 1911, p. 487. 
in colloidal chemistry, were shown also to exercise their influence upon the electric properties of membranes. Höber's studies furnish valuable experimental data, and the important question at present is whether these phenomena can be best explained in terms of Höber's theory of permeability or by the theory of forces at the phase boundary. Cremer ${ }^{18}$ correlated the variable permeability to ions in Ostwald's sense with the ionic mobilities, and regarded these potentials as being analogous to diffusion potentials. He identified the more diffusible ion as that ion which has a great mobility in the medium into which it penetrates. It was also Cremer who first succeeded in arranging experimentally a two-phase chain without the use of metals, such as:

$$
\begin{array}{c|c|c|c|}
\begin{array}{c}
\text { Aqueous } \\
\text { solution }
\end{array} & \text { Nitrobenzene } & \begin{array}{c}
\text { Nitrobenzene } \\
+ \text { picric acid }
\end{array} & \begin{array}{l}
\text { Aqueous } \\
\text { solution }
\end{array}
\end{array}
$$

In order to explain the observed potential differences in terms of Cremer's theory it. would be necessary to assume incredibly great differences in the mobilities of the several ions involved.

The phase boundary theories discussed in the preceding sections represent a development the way to which had been paved by Nernst's ${ }^{19}$ electromotive theory of metal electrodes. The experimental and theoretical investigations along this path were carried out in a most thorough manner by Haber, ${ }^{20}$ as well as previously by Cremer, although with not quite the same interpretation. These investigations were followed by those of Beutner referred to above and which represent a distinct step in advance, inasmuch as they can be directly applied to the interpretation of bioelectric phenomena. The place of origin of the electromotive forces is, according to the latter author's conclusions, at the boundary surface lying between aqueous phases and the lipoidal membranes of living cells and tissues. And, indeed, all such electric phenomena become easily explicable by merely assuming that such membranes repre-

${ }^{18}$ M. Cremer, Zeitschr. f. Biol. 47, 1 (1906); Nagels Handb. d. Physiol. 4, 868 (1909).

19 W. Nernst, Zeitschr. f. physikal. Chem. 9, 140 (1392); Nernst and Riesenfeld, Ann. d. Physik. 8, 600 (1902).

${ }^{20}$ F. Haber, Ann. d. Physik. 25, 917 (1905); Haber and Klemensiewicz, Zeitschr. f. physik. Chem. 67, 355 (1909). 
sent non-aqueous oil-phases containing oil-soluble organic acids. In order to prove Beutner's theory it is necessary to show that on varying the kind and the concentration of the electrolytes in the aqueous phase the resulting changes in the E.M.F. are qualitatively and quantitatively the same as those obtained in artificially reproduced oil-chains containing an acid (or acidified) oil-phase.

The electromotive forces observed in physiological systems must possess the following properties in order to fulfill the conditions of Beutner's theory:

1. A potential difference also must be observed between two uninjured places when they are connected by means of solutions of an electrolyte of different concentrations. This is shown in the following experiments:

(a) With differing concentrations of the same electrolyte.

The E.M.F. between two uninjured parts of an apple were measured, with the following solutions being used as connecting liquids:

\begin{tabular}{|c|c|c|c|}
\hline & & E.M.F. obserced & $\begin{array}{l}\text { Calculated maximal } \\
\text { concentration effect }\end{array}$ \\
\hline$/ / 10$ & $\mathrm{NaCl}$ and $M / 10$ & $\mathrm{NaCl} \cdot 0$ & \\
\hline$/ / 10$ & $\mathrm{NaCl}$ and $M / 50$ & $\mathrm{NaCl} \ldots 0.029-0.024$ volts & \\
\hline $1 / 50$ & $\mathrm{NaCl}$ and $M / 250$ & $\mathrm{NaCl} \ldots 0.042-0.036$ volts & 0.040 volts \\
\hline$/ 250$ & $\mathrm{NaCl}$ and $M / 1250$ & $\mathrm{NaCl} \ldots$ 0.041-0.038 volts & \\
\hline
\end{tabular}

showing that the more dilute solution is always positive.

The agreement with the results obtained from artificial oil-chains is so complete that even with the higher salt concentrations, the concentration effect is well below the theoretical maximal effect obtainable. With the more dilute solutions, the theoretical maximal effect is reached.

With $\mathrm{NaBr}$ the concentration effect was more pronounced.

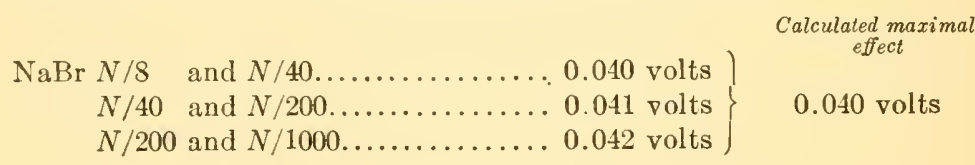

The concentration effect proved to be reversible whenever no permanent injury was inflicted upon the apple peel by the stronger salt solutions. 
With other materials the effect produced was less and not so regular, for example.

Turtle carapace:

KCl $N / 10$ and $N / 50 \ldots \ldots \ldots \ldots \ldots \ldots, 0.010$ volts

$N / 50$ and $N / 250 \ldots \ldots \ldots \ldots \ldots \ldots .0 .015$ volts

$N / 250$ and $N / 1250 \ldots \ldots \ldots \ldots \ldots 0.021$ volts

Calculated maximal effect

0.040 volts

Human finger nails:

$\mathrm{NaCl} 10 \mathrm{~N} / \mathrm{s}$ and $N / \mathrm{S} \ldots \ldots \ldots \ldots .0 .017$ volts

$N / 8$ and $N / 80 \ldots \ldots \ldots \ldots .0 .023$ volts

$N / 80$ and $N / 800 \ldots \ldots \ldots \ldots 0.028$ volts

0.0577 volts

$N / 800$ and $N / 8000 \ldots \ldots \ldots . .0 .030$ volts

In muscles a much smaller effect was obtained:

$\mathrm{KCl} N / S$ and $N / 40 \ldots \ldots \ldots \ldots \ldots . .002$ volts

$N / 40$ and $N / 200 \ldots \ldots \ldots \ldots \ldots \ldots, 0.006$ volts

$N / 200$ and $N / 1000 \ldots \ldots \ldots \ldots \ldots .000 .0 . \ldots$ volts

0.040 volts

(b) An E.M.F. must also be produced when two uninjured places are connected by means of solutions of the same concentration but of different electrolytes. The influence of the ions in the negative direction must follow in the same series sequence as in the "oilchains." This point had already been demonstrated in Höber's studies, which, however, could not at the time be interpreted in the sense of the later experiments. It was shown that when the connecting fluid was varied at an uninjured place, the negative effect of the anions was expressed in the series:

$$
\mathrm{SO}_{4}^{-} \quad \mathrm{Cl}^{-} \quad \mathrm{Br}^{-} \quad \mathrm{I}^{-} \quad \mathrm{NO}_{3}^{-} \quad \mathrm{SCN}^{-}
$$

and of the cations:

$$
\mathrm{K}^{+} \mathrm{Na}^{+} \mathrm{Ba}{ }^{++} \mathrm{Cu}^{++} \mathrm{Mg}^{++}
$$

Furthermore, for a closer conception of the phenomenon it was necessary to find out whether the oil-layer represented by the apple peel is a single oil phase, such as

$$
\text { salt solution | oil | salt solution }
$$

which would correspond to

$$
\text { salt solution } \mid \text { apple peel } \mid \begin{gathered}
\text { Free fluid of } \\
\text { apple pulp }
\end{gathered}
$$


or, whether different oil phases were present in layers, as

$$
\text { salt solution | oil I | oil II | salt solution }
$$

such as, for example,

Salt solution $\left|\begin{array}{c|c}\text { Nitrobenzene } \\ + \text { picric acid }\end{array}\right| \begin{gathered}\text { Nitrobenzene } \\ \text { without or with } \\ \text { less picric acid }\end{gathered} \mid$ Salt solution

corresponding to, in the case of the apple,

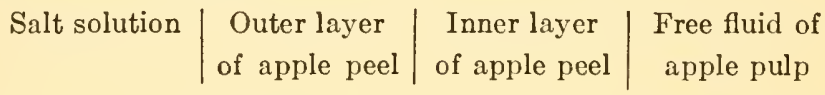

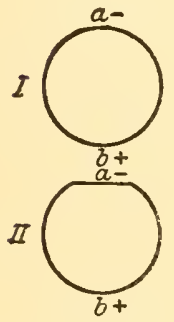

The following of Beutner's experiments answered this question. Two places on the apple were connected by means of $N / 50 \mathrm{KCl}$ solution (fig. 26). In the case of the uninjured apple (I) the E.M.F. was zero, of course. With a slight injury (II), a current of 0.041 volt was produced. With further injury (III) the effect remained unchanged. When the injury or hollowing out was carried on until but $0.25 \mathrm{~cm}$. of pulp remained (IV), the E.M.F. sank to 0.020 volt. And, finally, with the peel alone, (V) an E.M.F. of but 0.01 volt remained.

On the other hand, when the concentration of the

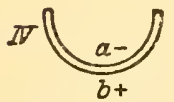
connecting fluid at the uninjured places was varied, the concentration effect appeared exactly the same as in the entirely uninjured apple.

The interpretation of this observation is only possible when it is assumed that the apple peel does not Fia. 26. (Taken represent a single oil-phase. Only the outermost from Beutner) layer of the peel may represent such a single oil phase, and hence, when placed between two salt solutions of the same concentration, it produced no current. Experiments II and IV above can only be explained by assuming a varying composition of the peel in its various layers, and that the outer layers contain in their oil phases an organic acid, which is absent or is present in a lesser concentration in the inner layers. 


\section{Potentials at precipitation membranes}

The theory of eell membrane potentials is based upon the analogy existing between an "oil"-phase and a eell membrane. But there is still an older model for eell membranes, which reproduced very well indeed its properties of permeability, namely M. Traube's precipitation membranes. Traube's membranes were adopted by Pfeffer in his well known eells for the qualitative and quantitative study of osmotic pressure and semipermeability. The fact that these copper ferrocyanide membranes showed the same impermeability for many dissolved substances as was assumed in the explanation of shrinking and swelling phenomena in cells placed into nonisotonic solutions, gave a great stimulus to the entire theory of semipermeability. And without this we could scarcely ever have arrived at the idea of a membrane which is permeable for eations but not for anions. The questions arise naturally whether such precipitation membranes can also be the seat of potential differences, whether these are analogous to the cell membrane potentials, and whether Beutner's theory is applicable to them, or, whether in this case the older idea of a specific permeability for definite ions is effeetive instead.

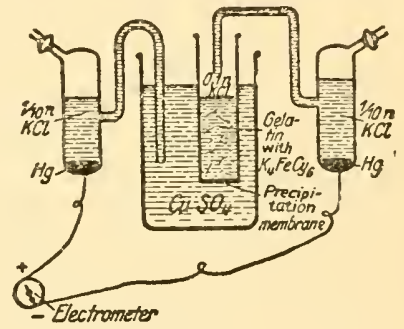

FIG. 27

Disregarding the older investigations of Brüning, ${ }^{21}$ Beutner studied such chains. His arrangement was the following (fig. 27):

A $M / 40$ warm solution of potassium ferrocyanide in 10 per eent gelatin solution was poured into a glass tube open at both ends, permitted to gel, and immersed in a beaker containing $M / 20 \mathrm{CuSO}_{4}$ solution. At the zone of contact a brown precipitation membrane of eopper ferrocyanide was immediately formed, barely pereeptible at first, but increasing in thickness with time. The upper end of the gelatin layer and the outer eopper solution were led off by means of calomel electrodes. An E.M.F. of $0.10-0.12$ volts was observed, the $\mathrm{CuSO}_{4}$ solution being positive. Then the composition of the outer solution was varied, keeping its osmotic pressure constant throughout by the addition of sugar in order not to destroy the

${ }^{21}$ Brüning, Pflügers Arch. f. d. ges. Physiol. 117, 409 (1907). 
membrane mechanically through the osmotic differences between the inner and the outer fluids. When the concentration of the $\mathrm{CuSO}_{4}$ only was varied, no definite constant change in the E.M.F. was observed. But when some $\mathrm{KCl}$ was added to a constant concentration of the $\mathrm{CuSO}_{4}$ the resulting E.M.F. values were observed to be definitely and irreversibly dependent upon the concentration of the added $\mathrm{KCl}$, as shown by the average figures given in table 34 .

But these are the exact values for a $\mathrm{KCl}$ concentration chain (0.040 volts).

The same results were obtained with $\mathrm{NaCl}, \mathrm{HCl}, \mathrm{NH}_{4} \mathrm{Cl}$, when the concentrations were varied, and on comparing chains containing different chlorides of the same concentration differences were again

TABLE 34

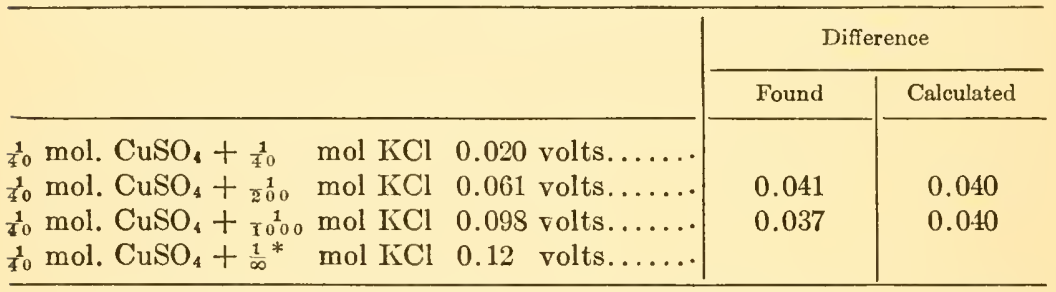

* An E.M.F. of $\infty$ was theoretically to be expected in this case. But small amounts of $\mathrm{K}$-salts $\left(\mathrm{K}_{2} \mathrm{SO}_{4}\right)$ diffuse through the membrane into the solution (see text below), hence the finite, but uncertain and irregular values for the E.M.F. observed.

found. In brief,-this form of chain is in every respect analogous to the chain.

$$
\begin{array}{c|c|c}
\text { Salt solution } & \text { Salicylic aldehyde } & \text { Salt solution } \\
\text { I } & \text { + salicylic acid } & \text { II }
\end{array}
$$

and it represents, for any cation, a reversible chain. The anions were shown to be without any effect, just as a chain whose middle phase consists of an acid oil. The analogy with such oil chains is so complete that it is permissible to apply the same theory to both. This theory may be stated as follows:

The formation of the cupric ferrocyanide occurs according to the equation:

$$
2 \mathrm{CuSO}_{4}+\mathrm{K}_{4} \mathrm{Fe}(\mathrm{CN})_{6}=2 \mathrm{~K}_{2} \mathrm{SO}_{4}+\mathrm{Cu}_{2} \mathrm{Fe}(\mathrm{CN})_{6}
$$


Thus there is also formed $\mathrm{K}_{2} \mathrm{SO}_{4}$, and it is present on both sides of the precipitation membrane. Although the membrane is considered impermeable for salts, yet it is certainly not so in the absolute sense. The fact that the membrane becomes thicker in the course of time shows that the salts do penetrate to a certain extent. As far as the potassium ferrocyanide solution is concerned the diffusion of the $\mathrm{K}_{2} \mathrm{SO}_{4}$ is immaterial. In the $\mathrm{CuSO}_{4}$ solution, on the other hand, it is only then unimportant, when it already contains a previously added large amount of a potassium salt, otherwise it is just the trace of $\mathrm{K}_{2} \mathrm{SO}_{4}$ which, on diffusing through, affects the E.M.F. of the chain appreciably. Thus our chain nay be schematized as:

\begin{tabular}{c|c|c}
$\begin{array}{c}\text { Solution } \\
\text { of } \mathrm{K}^{+} \text {ions }\end{array}$ & Membrane & $\begin{array}{c}\text { Solution } \\
\text { of } \mathrm{K}^{+} \text {ions } \\
\mathrm{c}_{1}\end{array}$
\end{tabular}

In order to explain the concentration effect it must be assumed that the membrane is a phase which also contains K-ions, but in an almost constant concentration, just as in the case of the acidcontaining oil phase. We may assume for this purpose that the membrane does not consist of entirely pure $\mathrm{Cu}_{2} \mathrm{Fe}(\mathrm{CN})_{6}$. These amorphous substances "carry along with them on precipitation a small portion of the soluble salts," or, there is formed some sort of combination of $\mathrm{Cu}_{2} \mathrm{Fe}(\mathrm{CN})_{6}$ with $\mathrm{K}_{2} \mathrm{SO}_{4}$. It can also be shown analytically that the $\mathrm{Cu}_{2} \mathrm{Fe}(\mathrm{CN})_{\epsilon}$ precipitate always contains alkali metal salts. It is uncertain whether, in this case, adsorption, solid solution or chemical combination is involved. But the very fact that the membrane holds $K$-salts in a firm way is sufficient in itself for our purposes. In the case of the acid-containing oil it was the affinity of the acid for the cations (or bases) which produced the almost constant concentration of cations in the portions of the oil phase in contact with the aqueous electrolyte solutions. In this case of precipitation membranes it is an affinity of the amorphous membrane substance for the salt which produces a similar effect. It is therefore possible to explain membrane potentials on the same basis as "oil-chain-potentials." Whether this explanation is the only correct one the future will show, but it does possess the advantage in that it can be easily correlated with other conceptions in a readily demonstrable way. We shall see in the next chapter that 
membranes may give rise to electric phenomena by an entirely different mechanism.

\section{Polarization phenomena at phase boundaries}

All of the above considerations of phase boundary potentials relate to the conditions where chemical equilibrium prevails, at least at the contiguous boundary layers. These potential differences are comparable to the E.M.F. of an open galvanic chain without a current. When such a galvanic chain is closed, the resulting current produces a greater or lesser change, according to the conditions, in the concentrations of the solutions, and along with it a change in the E.M.F. which is designated as polarization. This polarization may result either from the current produced by the E.M.F. of the chain itself or from a current sent out by an external E.M.F. through the chain. In the same way polarization, and hence a change in the phase boundary potential, may oceur in phase boundary chains either when they are shor't circuited for a longer period of time, or when an external current is sent through them. Nernst and Riesenfeld ${ }^{22}$ furnished the theory and the discussion of these polarization phenomena:

The arrangement, from left to right is as follows: (1) water saturated with phenol, (2) phenol saturated with water, (3) water saturated with phenol again; an electrolyte is distributed among these phases in a state of equilibrium, and, furthermore, for the sake of simplicity it is assumed that the phase boundary potential is zero. An electric current is sent through this system from left to right for a period of time, until one faraday (96,500 coulombs) has flowed through every cross section. The dissolved electrolyte consists of two univalent ions whose relative speeds are $u$ for the cation and $\mathrm{v}$ for the anion. In general the value of $\mathrm{u}$ in water will not be the same as in phenol, hence let us designate it as $u_{1}$ in water and $u_{2}$ in phenol; and likewise we shall differentiate $v_{1}$ from $v_{2}$. Let us consider any given cross section of the path of the current in the water. We find that the fraction of cations concerned in the transport of one faraday is $\frac{u_{1}}{u_{1}+v_{1}}=n_{1}$. This fraction is known as the transference number of the cation. Likewise the fraction $\frac{\mathrm{v}_{1}}{\mathrm{u}_{1}+\mathrm{v}_{\mathbf{1}}}$

${ }^{22}$ Nernst and Riesenfeld, Ann. d. Physik [4] 8, 600 (1902). 
$=\mathrm{m}_{1}$ of the anions is involved. Hence $\mathrm{n}_{1}+\mathrm{m}_{1}=1$. On the other hand, in the phenol the distribution is $\frac{u_{2}}{u_{2}+v_{2}}=n_{2}$ for the cations, and for the anions $\frac{\mathrm{v}_{2}}{\mathrm{u}_{2}+\mathrm{v}_{2}}=\mathrm{m}_{2}$, and again, $\mathrm{n}_{2}+\mathrm{m}_{2}=1$.

Let us now consider that cross section of the left hand water phase which is in direct contact with the phenol phase. Through this cross section the cations are moving from left to right, and the anions from right to left. In this cross section $n_{1}$ cations are moving inward from the left and $n_{2}$ cations outward to the right. If $n_{1}>n_{2}$ then $n_{1}-n_{2}$ cation will accumulate in this cross section. On the other hand, in the same cross section $m_{2}$ anions are moving from the right and $m_{1}$ anions to the left. When $n_{1}>n_{2}$, then $m_{1}$ must be $<m_{2}$, and hence $m_{2}-m_{1}$ anions accumulate in this cross section. Altogether $n_{1}-n_{2}$ cations and $m_{2}-m_{1}$ anions accumulate. But since $m_{2}=1-n_{2}$ and $m_{1}=1-n_{1}$, therefore, $m_{2}-m_{1}=n_{1}-n_{2}$, i.e., the total electrolyte accumulated in this cross section amounts to $2\left(n_{1}-n_{2}\right)$. The same consideration may be applied to the boundary layer of the phenol phase, in which we shall find a corresponding diminution of the electrolyte in the cross section. The same process occurs in the two boundary layers on the right hand side. Thus we find that the current produces an increase in electrolyte concentration in the water and a decrease in the phenol phase in both boundaries. Because of the spontaneous diffusion immediately arising between portions of solutions of unequal concentration, the changes of concentration at the boundary surfaces actually observed do not quite reach the expected values. When, reversely, $\mathrm{n}_{1}<\mathrm{n}_{2}$, we obtain an increase of concentration in the phenol and a decrease in the water layer. If the phase boundary potential was equal to zero at the beginning, it can no longer remain 0 after the passage of the current, because of the above changes in concentration at the boundaries. Thus a potential difference arises as a polarization phenomenon at the boundary surfaces. The two oppositely directed potentials of the two boundaries constitute the electromotive force of the polarization, which, in turn, must diminish the electric current, just as in the polarization of an electrolyte solution between two platinum electrodes. 


\section{CHAPTER IX}

\section{Membrane Potentials}

\section{SUMMARY OF CONTENTS}

When two aqueous electrolyte solutions are separated by a membrane which is impermeable for one of the ionic species present, a potential difference arises between the two solutions, and also an unequal distribution of the ions, including even the freely diffusible ions, ensues. This effect diminishes with increasing concentrations of the electrolytes. The relation of these potentials to the phase boundary potentials described in the preceding chapter is discussed.

\section{The membrane as the cause of a potential}

The phase boundary potentials which we have just considered are closely related to certain potential differences which we shall designate as membrane potentials.

As was stated in a preceding chapter (see page 210), Wilhelm Ostwald suggested the idea that the impermeability of membranes for any ionic species may give rise to potential differences. This theory was first fully developed by Donnan ${ }^{1}$ whose investigations have proved to be exceedingly fruitful. Donnan's derivation will be first shown for the simplest possible case. Let us take a solution containing only a Na-salt of an acid, the anions of this acid as well as its undissociated molecules being unable to penetrate the membrane, and let $\mathrm{R}^{-}$represent this anion. Let us take a second solution containing only $\mathrm{NaCl}$, and interpose between the solutions a membrane shown by a vertical line in the diagram:

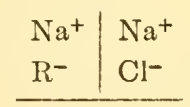

(1) (2)

${ }^{1}$ F. G. Donnan, Zeitschr. f. Elektrochemie, 17, 572 (1911). For the most recent account in English see F. G. Donnan. The Theory of Membrane Equilibria. Chemical Reviews, 1, 73 (1924). 
This initial state is soon altered through diffusion, and, considered qualitatively, results in the following redistribution of ions:

\begin{tabular}{l|l}
$\mathrm{Na}^{+}$ & $\mathrm{Na}^{+}$ \\
$\mathrm{R}^{-}$ & \\
$\mathrm{Cl}^{-}$ & $\mathrm{Cl}^{-}$ \\
\hline
\end{tabular}

(1) (2)

Since electroneutrality must be maintained in the solution on both sides of the membrane, it follows that, after the diffusion equilibrium had been established, $\left[\mathrm{Na}^{+}\right]_{1}=\left[\mathrm{R}^{-}\right]+\left[\mathrm{Cl}^{-}\right]_{1}$ and $\left[\mathrm{Na}^{+}\right]_{2}=\left[\mathrm{Cl}^{-}\right]_{2}$, and that neither $\left[\mathrm{Na}^{+}\right]_{1}=\left[\mathrm{Na}^{+}\right]_{2}$ nor $\left[\mathrm{Cl}^{-}\right]_{1}=\left[\mathrm{Cl}^{-}\right]_{2}$. This equilibrium is characterized by the fact that the maximal work which could be gained from the reversible and isothermic transport of a very small amount of Na-ions in one direction must be equal to the work expended in the reversible transport of the Cl-ions in the same direction. In other words, the algebraic sum of the work, $\delta \mathrm{A}$, which can be obtained from the simultaneous transport of a very small amount, $\delta \mathrm{n}$, of $\mathrm{Na}$-ions and of the same amount of Cl-ions must be equal to zero. The work which can be gained from the transport of $\delta \mathrm{n}$ moles of $\mathrm{Na}^{+}$, involving the change of concentration from $\left[\mathrm{Na}^{+}\right]_{1}$ to $\left[\mathrm{Na}^{+}\right]_{2}$ is $=\delta \mathrm{n} \times \mathrm{RT} \ln \frac{\left[\mathrm{Na}^{+}\right]_{2}}{\left[\mathrm{Na}_{1}\right]_{1}}$. The same condition being true for the Cl-ions, after the establishment of equilibrium we should have

$$
\delta \mathrm{n} \times \mathrm{RT} \ln \frac{\left[\mathrm{Na}^{+}\right]_{2}}{\left[\mathrm{Na}^{+}\right]_{1}}+\delta \mathrm{n} \times \mathrm{RT} \ln \frac{\left[\mathrm{Cl}^{-}\right]_{2}}{\left[\mathrm{Cl}^{-}\right]_{1}}=\mathrm{O}
$$

Hence it follows that

$$
\left[\mathrm{Na}^{+}\right]_{2} \times\left[\mathrm{Cl}^{-}\right]_{2}=\left[\mathrm{Na}^{+}\right]_{1} \times\left[\mathrm{Cl}^{-}\right]_{1}
$$

Certain difficulties which Donnan encountered in regard to the possible presence of undissociated molecules may be considered today as having been overcome. These difficulties were due to the inexact figures concerning the degree of dissociation of strong electrolytes obtained from calculations based on conductivity data. Therefore, we need not at present dwell at any greater length upon these difficulties.

Since in solution (2) we must have $\left[\mathrm{Na}^{+}\right]_{2}=\left[\mathrm{Cl}^{-}\right]_{2}$, but in solution (1) $\left[\mathrm{Na}^{+}\right]_{1}$ cannot be $=\left[\mathrm{Cl}^{-}\right]_{1}$, it can be stated that

$$
\left[\mathrm{Na}^{+}\right]_{1} \times\left[\mathrm{Cl}^{-}\right]_{1}=\left[\mathrm{Na}^{+}\right]_{2}^{2}=\left[\mathrm{Cl}^{-}\right]_{2}^{2}
$$


For the sake of preserving the continuity of argument developed in the preceding chapter let us choose an interpretation of this phenomenon somewhat different from Donnan's.

Let us imagine two electrolyte solutions separated by a membrane which is impermeable to one of the ionic species present. Let, for instance, the membrane be one of collodion and let one of the solutions be a $\mathrm{KCl}$ solution and the other of the chloricle of a cation which cation cannot diffuse through the membrane. A process of diffusion ensues leading to a certain state of equilibrium. But the above non-penetrating cation cannot take part in this diffusion. If the system is initially

\begin{tabular}{|c|c|c|}
\hline Colloid-chloride & & $\mathrm{KCl}$ \\
\hline $\begin{array}{c}\text { (dissociated into } \\
\text { colloid-cation }{ }^{+}+\mathrm{Cl}^{-} \text {) }\end{array}$ & Membrane & $\begin{array}{c}\text { (dissociated into } \\
\left.\mathrm{K}^{+}+\mathrm{Cl}^{-}\right)\end{array}$ \\
\hline
\end{tabular}

diffusion will at once occur through the membrane. ${ }^{2}$ K-ions will diffuse from right to left, Cl-ions will diffuse, according to the conditions, from right to left, or reversely. But there is no possibility that the concentrations of $\mathrm{K}^{+}$become the same in both solutions, nor could that happen for the Cl-ions. For, if this were the case, then the solution on the left would contain, because of the presence of the colloid-cations, more cations than anions, which state would violate the law of electroneutrality. It is more likely that the colloidcations will hold back a part of the Cl-ions. For in the state of equilibrium the total sum of cations in the solution on the left (colloid cations $\left.+\mathrm{K}^{+}\right)$must be equal to its content of anions $\left(\mathrm{Cl}^{-}\right)$, and, similarly, in the solution on the right side, the concentration of the K-ions must equal that of its Cl-ions. The inequality of the concentrations of the oppositely charged ions can be but immeasurably small, and it effects a potential difference on both sides of the membrane. In the first place then, we find an analytically demonstrable difference between the Cl-ion concentrations in the solutions, and, secondly, we also find a potential difference. These two phenomena are definitely related to each other, and this relation can be calculated.

Thus, if after the establishment of equilibrium, we should immerse in each of the two solutions, a reversible $\mathrm{Cl}^{-}$electrode (i.e., a gas

2 The term "colloid" in the above scheme does not in this case signify that the substance is not in molecularly dispersed solution, but it only signifies that it does not diffuse through the membrane. 
$\mathrm{Pt}-\mathrm{Cl}_{2}$ electrode, or a $\mathrm{Hg}-\mathrm{HgCl}$ eiectrode which is reversible for $\left.\mathrm{Cl}^{-}\right)$and unite the electrodes by a metal conductor, we shall find that no current can flow through this system, since it is in equilibrium. But the electrode potentials are not equal to each other. Thus, if in the final state of equilibrium the $\mathrm{Cl}^{-}$concentration on the left is $c_{1}$ and on the right is $c_{2}$, then the potential difference between the two electrodes is equal to $\mathrm{RT} \ln \frac{\mathrm{c}_{1}}{\mathrm{c}_{2}}$. In order that the entire system (or this chain) be entirely devoid of an electric current flowing through it, we must assume that necessarily a potential amounting to - RT $\ln \frac{c_{1}}{c_{2}}$ be present at the membrane.

If we should now assume that in addition to $\mathrm{KCl}$ also $\mathrm{HCl}$ is present, then a different state of equilibrium will ensue. This equilibrium can also be evaluated. If we designate again the concentrations of $\mathrm{Cl}^{-}$in the left and right solutions $\mathrm{c}_{1}$ and $\mathrm{c}_{2}$ respectively (which are now different, of course, from the $c_{1}$ and $c_{2}$ used in the preceding example), the potential difference will be $-R T \ln \frac{c_{1}}{c_{2}}$. But we can use hydrogen electrodes now, and even then the chain will not yield any electric current. But this may only be true if the membrane potential is equal to $R T \ln \frac{h_{1}}{h_{2}}$, where $h_{1}$ and $h_{2}$ represent the $\left[\mathrm{H}^{+}\right]$of the two solutions. The same conditions will prevail in the presence of any of the common ions along with our non diffusible ion. And thus by determining, after equilibrium has been attained in the system, the concentrations of any of its component ions, for instance that of the H-ions, which are always present, and which are easily determined, we can calculate from it the following:

1. The membrane potential.

2. The distribution of any of the ionic species between the two solutions.

When all the ions present are univalent, and letting the ratio of the $\mathrm{H}^{+}$-concentrations, of that in the left to that in the right solution be $\gamma: 1$, then

1. The potential $=\mathrm{RT} \ln \gamma$.

2. The ratio of the concentrations of any of the positive diffusible ions, that in the left to that in the right solution, is likewise = $\gamma$, and for the negative ions it is $=\frac{1}{\gamma}$. 
The simplest case to be had is that in which but one diffusible electrolyte is present, such as

\begin{tabular}{c|c} 
Colloid base \\
$\mathrm{HCl}$
\end{tabular} \mid $\mathrm{HCl}$

According to the principle developed above the right to left ratio of the $\mathrm{H}^{+}$-concentrations, must be equal to the left to right $\mathrm{Cl}^{-}$-concentration ratio, thus:

$$
\frac{\left[\mathrm{H}^{+}\right]_{2}}{\left[\mathrm{H}^{+}\right]_{1}}=\frac{\left[\mathrm{Cl}^{-}\right]_{1}}{\left[\mathrm{Cl}^{-}\right]_{2}}
$$

But since on the right side $\left[\mathrm{H}^{+}\right]_{2}=\left[\mathrm{Cl}^{-}\right]_{2}$, it follows that

$$
\left[\mathrm { H } ^ { + } \left[_{2}^{2}=\left[\mathrm{Cl}^{-}\right]_{2}^{2}=\left[\mathrm{H}^{+}\right]_{1} \times\left[\mathrm{Cl}^{-}\right]_{1}\right.\right.
$$

Let us now see how we can define more closely the distribution of ions in the simplest possible case. Let us take this system: the solution inside of a collodion thimble is that of a chloride of a nondiffusible, but molecularly dispersed, base in concentration a. Also, the $\mathrm{H}^{+}$and $\mathrm{Cl}^{-}$ions are present in this inner solution in the concentrations $\mathrm{h}_{1}$ and $\mathrm{cl}_{1}$. In the outer solution we have $\mathrm{HCl}$ in the concentration $\mathrm{h}_{2}=\mathrm{cl}_{2}$, but no "colloid" is present. The electrolytes are assumed to be completely dissociated. The values $h_{2}$ $=\mathrm{cl}_{2}$ are known while $\mathrm{h}_{1}$ and $\mathrm{cl}_{1}$ are taken as the unknown values. The following relations exist:

$$
\begin{aligned}
\mathrm{a}+\mathrm{h}_{1} & =\mathrm{cl}_{1} \\
\mathrm{~h}_{2} & =\mathrm{cl}_{2} \\
\frac{\mathrm{h}_{1}}{\mathrm{~h}_{2}} & =\frac{\mathrm{cl}_{2}}{\mathrm{cl}_{1}}
\end{aligned}
$$

and also

$$
\begin{aligned}
& \mathrm{a}+\mathrm{h}_{1}=\mathrm{cl}_{1} \\
& \mathrm{~h}_{1} \cdot \mathrm{cl}_{1}=\mathrm{cl}_{2}{ }^{2}
\end{aligned}
$$

From (1) and (2) the following quadratic equation for $\mathrm{cl}_{1}$ is obtained:

$$
\begin{aligned}
& \mathrm{cl}_{1}{ }^{2}-\mathrm{a} \mathrm{cl}_{1}-\mathrm{cl}_{2}^{2}=0 \\
& \mathrm{cl}_{1}=\frac{\mathrm{a}}{2}+\sqrt{\frac{\mathrm{a}^{2}}{4}+\mathrm{cl}_{2}^{2}}
\end{aligned}
$$


That only the positive root is significant can be seen by setting $\mathrm{a}=0$, when $\mathrm{cl}_{1}=\mathrm{cl}_{2}$, and not $\mathrm{cl}_{1}=-\mathrm{cl}_{2}$.

By substitution in (1) we obtain

$$
\mathrm{h}_{1}=-\frac{\mathrm{a}}{2}+\sqrt{\frac{\mathrm{a}^{2}}{4}+\mathrm{cl}_{2}^{2}}
$$

Thus we see that $\mathrm{cl}_{1}>\mathrm{h}_{1}$, i.e., the positively charged colloid ion simultaneously attracts the negative $\mathrm{Cl}$-ions and repels the positive $\mathrm{H}$-ions outward through the membrane.

If we assume that in addition to $\mathrm{HCl}, \mathrm{NaCl}$ is also present in the solution, then in the inner solution the ions are in the concentrations a, $\mathrm{h}_{1}, \mathrm{cl}_{1}, \mathrm{n}_{1}$ (where $\mathrm{n}_{1}$ is the $\mathrm{Na}^{+}$-concentration); and in the outer solution $\mathrm{h}_{2}, \mathrm{el}_{2}, \mathrm{n}_{2}$. And now we have the following relationships.

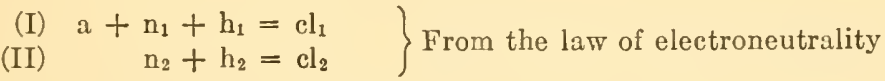

$$
\left.\begin{array}{l}
h_{1}: h_{2}=c_{2}: c_{1} \\
h_{1}: h_{2}=n_{1}: n_{2}
\end{array}\right\} \text { From Donnan's law of ionic distribution }
$$

We shall now solve these equations for $h_{1}$, so that $h_{1}$ appears as a function only of a and other values carrying the subscript 2 . This is done by solving (III) for $h_{1}$, eliminating $\mathrm{cl}_{1}$ by means of (I) and $n_{1}$ by means of (IV) which results in

$$
\mathrm{h}_{1}=\frac{\mathrm{el}_{2} \cdot \mathrm{h}_{2}}{\mathrm{a}+\frac{\mathrm{h}_{1}}{\mathrm{~h}_{2}} \cdot \mathrm{n}_{2}+\mathrm{h}_{1}}
$$

This in turn yields a quadratic equation for $\mathrm{h}_{1}$

$$
h_{1}=-\frac{a \cdot h_{2}}{2 \mathrm{cl}_{2}}+\sqrt{\left(\frac{\mathrm{a} \cdot \mathrm{h}_{2}}{2 \mathrm{cl}_{2}}\right)^{2}+\mathrm{h}_{2}{ }^{2}}
$$

And hence,

$$
\frac{\mathrm{h}_{1}}{\mathrm{~h}_{2}}=-\frac{\mathrm{a}}{2 \mathrm{cl}_{2}}+\sqrt{1+\left(\frac{\mathrm{a}}{2 \mathrm{cl}_{2}}\right)^{2}}
$$

From the last equation and (III) we can at once derive the ratio $\mathrm{cl}_{1} / \mathrm{cl}_{2}$, and, similarly by using (IV), also $\mathrm{n}_{1} / \mathrm{n}_{2}$. Furthermore, we can now express the total ionic distribution and the membrane poten- 
tial as a function of two variables, a "colloid" ion concentration), and $\mathrm{Cl}_{2}$ (which is in general the concentration in the outer solution of any ion having a charge opposite to that of the "colloid"-ion).

Let us now consider the relation of the ratio $h_{1} / h_{2}$ to a. From equation (5) we see that when $a=0$ (i.e., in the absence of a "colloid" ion), $\mathrm{h}_{1} / \mathrm{h}_{2}=1$, and that therefore the potential $=\mathrm{RT} \ln 1$ $=0$; and that the greater the value of a the greater the difference between $h_{1} / h_{2}$ and 1 , and thus the greater the resulting potential.

Now for the relation of $h_{1} / h_{2}$ to the value of $\mathrm{cl}_{2}$. It is such that the ratio $h_{1} / h_{2}$ increases, without forming a maximum or minimum, with increasing values of $c_{2}$. In fact, the greatest value for $h_{1} / h_{2}=1$ is when $\mathrm{cl}_{2}=\infty$, and its lowest value is 0 when $\mathrm{cl}_{2}=0$. For, if we. write (5) in the form

$$
y=-x+\sqrt{x^{2}+1}
$$

and assume that $\mathrm{x}$ is very large (i.e. that $\mathrm{cl}_{2}$ is very small) in respect to 1 , then we can express the term $\sqrt{x^{2}+1}$, by developing it in a binomial series, as

$$
x+\frac{1}{2 x}-\frac{1}{8 x^{3}} \ldots \ldots, \text { and hence, } y=\frac{1}{2 x}+\frac{1}{8 x^{3}} \ldots \ldots .
$$

For $\mathrm{x}=\infty$ (i.e., for $\mathrm{cl}_{2}=0$ ), $\mathrm{y}$ (i.e., $\mathrm{h}_{1} / \mathrm{h}_{2}$ ) becomes $=0$.

It is quite easy, therefore, in a serial experiment, keeping the value of a constant, to increase the outer concentration of the Cl-ion, $\mathrm{cl}_{2}$, by simply adding to the whole system $\mathrm{HCl}$ or any chloride. Hence it follows that:

1. The value of the ratio $h_{1} / h_{2}$ approaches 1 (the potential approaches 0 ) with the addition of $\mathrm{HCl}$.

2. The same effect is obtained on the addition of any chloride, e.g., $\mathrm{NaCl}$. This leads to the following physiologically important. conclusion:

All membrane potentials are decreased by the addition of neutral salts; and in colloidal solutions rich in electrolytes only very small membrane. potentials are developed.

It will be interesting to see just what potentials are to be expected under certain assumptions. If the concentration of the molecularly dispersed non-diffusible cation is taken as a $=0.01 N$, then by applying equation (5) (page 225) we shall find in the outer solution the 
different values for $\mathrm{cl}_{2}$ shown in table 35 . When it is assumed that a $=0.1 \mathrm{~N}$, the values shown in table 36 will be obtained.

When the "colloid" is of the nature of an anion, then all of the above derivations may be directly applied by simply substituting oh for $h$ and $n$ for cl. Since $o h_{1} / \mathrm{oh}_{2}=h_{2} / h_{1}$ equation (5) above becomes

$$
\frac{\mathrm{h}_{2}}{\mathrm{~h}_{1}}=-\frac{\mathrm{a}}{2 \mathrm{n}_{2}}+\sqrt{1+\left(\frac{\mathrm{a}}{2 \mathrm{n}_{2}}\right)^{2}}
$$

TABLE 35

\begin{tabular}{l|c|c}
\hline $\mathrm{cl}_{2}$ (normal) & $\mathrm{h}_{1} / \mathrm{h}_{2}$ & $\pi$ millivolts \\
\hline $\mathbf{1}$ & 0.995 & 0.1 \\
$\mathbf{0 . 1}$ & 0.95 & 1.3 \\
$\mathbf{0 . 0 1}$ & 0.62 & 12.2 \\
$\mathbf{0 . 0 0 1}$ & 0.10 & 58 \\
$\mathbf{0 . 0 0 0 1}$ & 0.01 & 116 \\
$\mathbf{0 . 0 0 0 0 1}$ & $\mathrm{ca} 0.001$ & $\mathrm{cr.174}$ \\
\hline
\end{tabular}

TABLE 36

\begin{tabular}{l|c|c}
\hline \multicolumn{1}{c|}{$\mathrm{cl}_{2}$} & $-\mathrm{h}_{1} / \mathrm{h}_{2}$ & $\pi$ millivolts \\
\hline 1 & 0.93 & 1.3 \\
0.1 & 0.62 & 12.2 \\
0.01 & 0.10 & 58 \\
0.001 & 0.01 & 116 \\
0.0001 & 0.001 & 174 \\
0.00001 & 0.0001 & 232 \\
\hline
\end{tabular}

and the two tables shown above retain their validity in this case by merely changing $h_{1} / h_{2}$ to $h_{2} / h_{1}$ and $\mathrm{cl}_{2}$ to $n_{2}$.

Let us now attempt to derive from the above consideration such useful physiological applications as may be possible at present. We shall take the case of a cell whose content represents a $0.01-0.1$ molar solution of protein, which, insofar as it is ionized, we shall consider as being moleculary dispersed. The protein, at the $\mathrm{pH}$ of the tissue fluids, must be assumed to be in the form of anions, insofar as it is ionized in general. The cell is surrounded by a membrane impermeable to protein and is bathed in a protein-free $\mathrm{NaCl}$ solution, approximately $0.1 \mathrm{~N}$. In such a case we should expect a membrane 
potential of between 1.3 and 12.2 millivolts. The latter value would represent the highest imaginable. However, such ideal conditions as: a 0.1 molar protein concentration; a complete molecular dispersion and ionization of the protein solution; absence of protein in the surrounding fluid, can barely be found in the living organism. Therefore it is quite questionable whether Donnan's theory of ionic distribution and of formation of potentials can, in this form, play an important rêle in the living organism. On the other hand, for certain experimental conditions in colloidal chemistry the theory is of the greatest importance, especially in the following respects.

It is not unconditionally requisite that the colloidal and the colloid-free solutions be separated by an actual membrane. Procter ${ }^{3}$ was the first to demonstrate that a piece of solid gelatin, immersed in an aqueous solution and permitted to assume a definite state of swelling, behaves in exactly the same manner. A solution is enclosed within the meshes of the gel, and the gel itself represents, according to the $\mathrm{pH}$ and because of its amphoteric character, a non-diffusible acid or base. Consequently, when a piece of gelatin swells in a dilute $\mathrm{HCl}$ solution, at equilibrium the $\mathrm{H}^{+}$-concentration must be greater in the outer solution than within the gel, and this difference becomes less upon the addition of $\mathrm{NaCl}$. This observation was verified experimentally by Jacques Loeb, ${ }^{4}$ and we shall return to this topic in our chapter on colloids.

\section{The relation of membrane potentials to phase boundary potentials}

If it appear's that the biological significance of these potentials is not very great for the living cell, it is yet possible that membrane potentials originating from another source may be of importance for the living organism. The investigations of many physiologists have led to the irlea that the membranes of various cells show a specifically selective permeability for different ions. R. Höber, especially, advanced the theory that cell membranes possess a general difference in permeability for cations and anions. Ever since the fundamental

${ }^{3}$ H. R. Procter, Journ. of the Chem. Soc. 105, 313 (1914); H. R. Procter and J. A. Wilson, Jnurn. of the Chem. Soc. 103, 307 (1916).

${ }^{4}$ Jacques Loeb, Journ. of Gen. Physinl. 3, 667; 3, 691; 3, 827; 4, 33; 4, 97 (1921): also J. Loeb. Proteins and the Theory of Colloidal Behavior. 2nd ed. New York, 1924. 
researches of Pfeffer and of de Tries on plant cells and of Hamburger on animal cells it has been assumed that most of the water soluble substances, such as particularly sugar and $\mathrm{NaCl}$, cannot penetrate the cell membrane. Those substances which do penetrate (urea, narcotics) are, according to Overton and Hans Meyer, lipoid-soluble. In later years this hypothesis has not been sustained. Many investigations have since demonstrated that anions diffuse freely through the membrane of blood corpuscles, and that only the cations are nondiffusible. This question cannot be very well discussed in greater detail at this juncture. But, if this theory is at all correct, then all conditions and prerequisites are present for the formation of membrane potentials. Only the non-diffusible ion is in this case not the protein-ion, but the common alkali metal cation. Since such cations are present in the tissue fluids in much greater molar concentrations than the proteins, it becomes quite possible that they give rise to considerable membrane potentials. The only difficulty lies in the fact that thus far it has not been found possible to construct a satisfactory model for the study of such processes, or to reproduce a membrane which would be impermeable to cations alone, in the same sense in which phase boundary potentials have been so successfully reproduced artificially. This leads us to the following conjecture:

If we recall Haber's glass chains or Beutner's "oil chains," it will be remembered that they are considered as chains "reversible only for cations or only for anions." We could restate this by saying that the oil-membrane is "permeable" only for cations (or only for anions). This impermeability is not by any means of the same lind as that of the collodion membrane for protein. In the latter case the impermeability is explained on the basis of a spatial disparity between the pores of the membrane and the diffusing molecules, while in the former case it is based upon the limited solubility of the ions of the aqueous solution in the medium of the "oil". In the former" case the membrane is represented as a system of very fine canals through which all the (non-colloidal) molecules, of the solvent as well as of the solutes, circulate freely, the solvent in the pores being the same (water) as in the two solutions. In the latter case the membrane is conceived of as a foreign phase interposed between the two solutions and representing a solvent totally different from those of the solutions on either side of it.

This brings us back to the first statement of this section in which 
the close relationship of phase boundary potentials and membrane potentials was suggested. A layer of an oil interposed between two aqueous phases behaves, under certain conditions, like a membrane impermeable to certain ions, and hence it should be possible to regard the ionic distribution at equilibrium in both ways. It seems, however, that the interpretation in terms of phase boundary potentials is the more comprehensive of the two. Donnan's interpretation should be limited to those cases in which the impermeability is actually determined by a lack of diffusibility (of a molecule); where the ultimate cause of the potential lies in the presence of a colloid in the aqueous solution; where the membrane may be imagined as a system of fine canals through which all substances (except the colloid) circulate freely: and where, especially, the solvent circulating in these canals is the same as in the adjacent solutions. In such a case Donnan's interpretation is quite irreplaceable, and as such it has assumed an important place in the theory of colloidal systems. 


\section{CHAPTER $\mathrm{X}$}

\section{Adsorption Potentials and Electrokinetic Phenomena}

\section{SUMMARY OF CONTENTS}

Adsorption is defined as that phenomenon in which a substance dissolved in one phase becomes concentrated at the boundary surface of an adjacent phase, irrespective of the forces causing this concentration. If such adsorption involves dissolved electrolytes, then the unequal adsorption of positive and negative ions may give rise to a potential difference at the boundary surface. By studying the adsorption of electrolytes on charcoal one may obtain an idea of the adsorbability of the different ions. That of the $H$-and $O H$-ions appears to be especially great. The adsorbing surfaces may be grouped, according to their capacity to bind $H$ or OH-ions, as acidoids, basoids or ampholytoids. This classification corresponds to that derived on the basis of chemical constitution for the water-soluble acids, bases and ampholytes. In the process of adsorption all cations compete with the $H$-ions, while all anions compete with the $O H$-ions. Because of an opposite exchange of ionogenic radicals between the adsorbent and the dissolved substances exchange-adsorptions arise, which may be considered almost entirely in terms of purely chemical phenomena. The adsorption of ions is the cause of the electric charge on the boundary surface. These potential differences become manifest in the phenomena of endosmosis or cataphoresis, whose history and theory will be reviewed. The tangential displacements of the double layers against each other are chiefly involved. The electrically charged or ionic layer moving in water carries along with it water particles. According to whether the water or the solid particles are fixed by mechanical means, cataphoresis or endosmosis ensues. The effect of the dissolved electrolytes upon the magnitude and direction of the endosinosis or the cataphoresis permits one to predict the magnitude and direction of the adsorption potentials, and the results correspond to those to be expected from the determination of the actual adsorption of ions. In this connection we find again that the adsorbents may be grouped into: always negative (acidoids), always positive (basoids) and those with a change- 
able sign of charge (ampholytoids). Among the acidoids there are some whose sign of charge can be reversed, if not by $H$-ions, then by trivalent cations. In the electrocndosmosis of water, the changes in the concentrations of dissolved electrolytes on both sides of the diaphragm are interrelated, especially the changes in $\mathrm{H}$-ion concentrations.

Another manifestation of adsorption potentials are the hydrodynamic potentials.

The adsorption potentials are clectromotively inactive. When they are led off to electric measuring instruments, in the same way as the previously described phase boundary potentials, then only such phase boundary potentials are manifested. The adsorption potential is only a stage in that potential difference whose totality represents the phase boundary potential. It is best, therefore, to represent the phase boundary potential not as an unstable potential difference, but rather as a gradual change of potential within a very thin but measurable layer.

Finally, it will be attempted to correlate Coehn's law with these phenomena, and the relation of boundary potentials to colloid chemistry will be discussed.

\section{Definition of adsorption}

The concept of adsorption potentials as developed below includes a whole series of electric phenomena which are of great importance in biology and are of no less interest to the pure physicist. The problems of this field are as yet even further removed from their ultimate solution than those presented above. It is possible that the method of presentation chosen will contribute towards further elucidation, insofar as it is unified in character and at the same time points out the existing gaps in our knowledge. Therefore, the whole method of presentation employed may be regarded as an attempt to fuse together facts and theories of varied origin into a single coherent structure. The future is to show of what developments such a structure is capable. The point of departure will be the phenomonon of adsorption, especially the adsorption of ions.

For this purpose it will be necessary to define adsorption in the sense in which we shall use this term. Different authors have given it different interpretations. For our purposes we shall define it as that process by which a substance accumulates at a boundary surface of two contiguous phases in a concentration higher than it exists in the interior of these two phases. The question of the forces 
causing this accumulation is deliberately left open, i.e., it is immaterial for the present whether it is caused by physical or by the so-called chemical forces. At the time when Rutherford's atomic model is constantly furnishing greater support for the common basis of the physical and chemical properties of inatter, it appears quite futile to attempt to differentiate between mechanical forces of adhesion and chemical attractions. We shall therefore use the term adsorption not in any sense of distinction from chemical union, and we shall take it to include cases which theoretical chemists would recognize as chemical reactions, as well as such cases which, to an investigator of the older school, would not convey the least suggestion of chemical affinity, such as, for example, the adsorption by charcoal of an alcohol dissolved in water. The important feature of our definition is that this binding process occurs at the boundary surface of two phases, and not in the interiors of these phases.

This definition of adsorption leads to a peculiar consequence. According to Gibbs' principle, surface-active substances dissolved in water must concentrate in the surface layer of the water, even though no special "adsorbent" is present, and even when the water is bound only by a gaseous space. In this case, according to our definition, the gaseous space would be the "adsorbent." While it is true that this designation is a more or less borrowed one, it cannot, nevertheless, be neglected. Thus let us take for an example two phases, $A$ and $B$, and dissolved in phase $\mathrm{A}$ is substance $\mathrm{C}$, which is either entirely insoluble in $\mathrm{B}$, or has reacher in $\mathrm{B}$ its equilibrium of distribution. Then the composition of the boundary layer will be determined by the effects of the following molecular interrelationships: 1 . those of the molecules $\mathrm{A}-\mathrm{A}, 2$. A - B;3. A - C; 4. B - B; 5. B - C; and 6. $\mathrm{C}-\mathrm{C}$. But when phase $\mathrm{B}$ (a gas) is practically without mass in comparison with $\mathrm{A}$, then the forces $\mathrm{A}-\mathrm{B}, \mathrm{B}-\mathrm{B}$, and $\mathrm{B}-\mathrm{C}$ need not be considered. In spite of this, and because of the interference of the remaining forces, there occurs a concentration of substance C in the surface layer of phase A. One could offer the definition:-in this case the adsorbent is lacking; hence an adsorbent is a substance which changes such a state of distribution. But since the amount of $\mathrm{C}$ accumulated in the free surface layer cannot be experimentally determined, practically it is preferable to choose for our point of departure that state in which the dissolved substance $\mathrm{C}$ is uniformly distributed throughout in phase $\mathrm{A}$, and to designate every accumu- 
lation in the surface layer as adsorption. If it is desired to differentiate such an adsorption as described above from the usual mode, it could be designated as "apparent adsorption." In these terms the empty space becomes also an adsorbent. And thus this definition facilitates the formal explanation of the phenomena of adsorption.

\section{The adsorption of electrolytes by charcoal}

The problem reduced to its simplest form relates to the adsorption of the individual ionic species. But since it is impossible to obtain a solution of an individual ionic species, and since we can only have a

TABLE 37

\begin{tabular}{l|l|l|l|l|l|l|l}
\hline & $\mathrm{Cl}^{\frac{1}{3}} \mathrm{SO}_{4}^{-}$ & $\mathrm{CNS}^{-}$ & $\mathrm{CH}^{-}$ \\
\hline
\end{tabular}

The adsorption of ions from $0.1 \mathrm{~N}$ solutions by charcoal

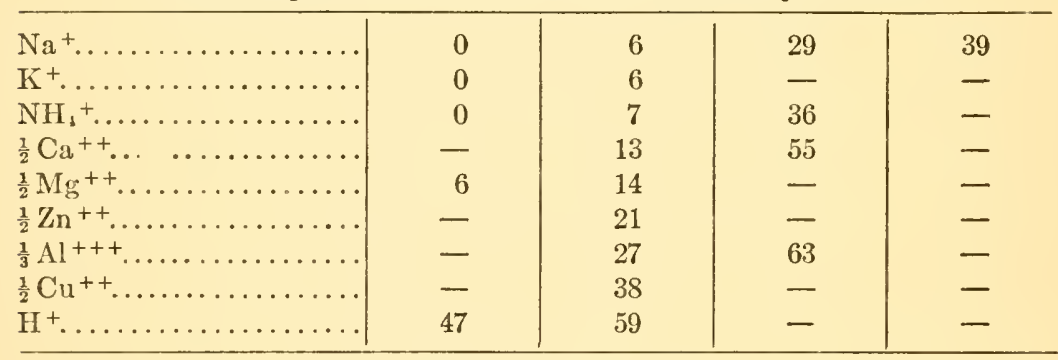

With $0.05 \mathrm{~N}$ solutions

\begin{tabular}{|c|c|c|c|c|}
\hline 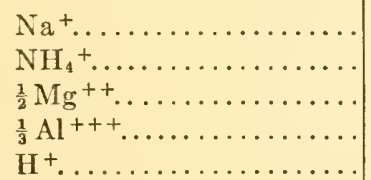 & $\begin{array}{r}0 \\
0 \\
16 \\
47 \\
70\end{array}$ & $\begin{array}{l}11 \\
11 \\
- \\
-\end{array}$ & $\begin{array}{l}35 \\
- \\
73 \\
-\end{array}$ & $\begin{array}{l}68 \\
- \\
-\end{array}$ \\
\hline
\end{tabular}

solution of one electrolyte, i.e., a mixture of a negatively charged and a positively charged species of ions, so that actually we can only investigate experimentally the adsorption of the entire electrolyte molecule, and any statement concerning the adsorption of a single ionic species, which completely escapes chemical analysis, can be made only by inference, and with greater or lesser certainty. Thus we find, for instance, in studying the adsorption of different sodium salts ( $\mathrm{NaCl}, \mathrm{NaBr}, \mathrm{NaI}$, etc.) certain quantitative differences. Now if we study the calcium salts of the same anions, we find other absolute values, but the relative differences in the series of anions are 
found to be the same as before. Hence we must conclude that the adsorption properties of an electrolyte are additive functions of those of its anions and cations. But also the nature of the adsorbent as well as of the solvent, has an influence upon the process of adsorption. Therefore, in an experimental study, we must vary the adsorbent, the cation and the anion. For the adsorbent we shall choose for the present charcoal, ${ }^{1}$ which is the prototype of the so-called chemically indifferent adsorbing agents, and we shall vary the anions and the cations.

After some preliminary experiments with Lachs, the author in collaboration with Rona ${ }^{2}$ has carried out a systematic series of investigations and has obtained the following results.

Of the neutral salts all, with the exception of the sulfates of the alkali metals, were adsorbed to an analytically demonstrable extent. In all cases of true neutral salts equal amounts of cation and anion were adsorbed, no measurable amount of hydrolytic splitting having occurred in the adsorption of true neutral salts. The adsorption of a salt appeared as an additive property of its ions, thus, for instance, all sulfocyanates were more strongly adsorbed than the corresponding chlorides.

Consequently the anions and cations can be arranged in series according to their adsorbabilities. In such series we can also include the $\mathrm{H}$ - and $\mathrm{OH}$-ions, by simply comparing the adsorption of $\mathrm{HCl}$ with that of other chlorides and by comparing the adsorption of $\mathrm{NaOH}$ with that of other Na-salts of equivalent concentrations. These series are

Cations:

\begin{tabular}{|c|c|c|c|}
\hline $\mathrm{Na}^{+}$ & & & Organic dye bases \\
\hline $\left.\mathrm{K}^{+}\right\}$ & $\mathrm{Ca}^{++} \mathrm{Mg}^{++}$ & $\mathrm{Al}^{+++} \mathrm{Cu}^{++}$ & $\mathrm{H}^{+}$ \\
\hline $\mathrm{NH}_{4}^{+}$ & & & $\mathrm{Ag}^{+}$ \\
\hline
\end{tabular}

Anions:

$\mathrm{SO}_{4}^{-} \quad \mathrm{Cl}^{-} \mathrm{Br}^{-} \mathrm{I}^{-} \mathrm{SCN}^{-} \mathrm{OH}^{-}$

Organic dye acids

${ }^{1}$ Blood charcoal will always be understood. Retort-, sugar- and other charcoals do not show the same uniform behavior.

${ }^{2}$ Lachs and Michaelis, Koll.-Z. 9, 275 (1911); Zeitschr. f. Elektrochemie 17, 1 and 917 (1911); Rona and Michaelis, Bioch. Zeitschr. 94, 240;97, 85; 103, 19 (1920); Michaelis and Rona, Bioch. Zeitschr. 94, 225; 97, 57; 102, 268 (1920): Kolloid-Zeitschr. 25, 225 (1919). 
The most striking aspect of these series is the relation to the electromotive tension series, the nobler the metal the more easily are its ions adsorbed. Also the valence has a certain infiuence. The higher oxidation forms of the ions $\left(\mathrm{Fe}^{+++}, \mathrm{Hg}^{++}\right.$) are exceptionally strongly adsorbable, as well as the salts of certain organic bases and acids, such as the dyes, quinine, etc. Among the halogen anions the discharge tension increases with increasing atomic weight. No clemonstrable difference in the adsorbabilities of the different alkali metal cations was observed. This fact was also confirmed by Hartieben. ${ }^{3}$

The relative adsorption of the salts, as it was also shown for nonelectrolytes, increases with the decreasing concentration of their solutions. Table 37, taken from the cited article by Rona and Michaelis, shows the results obtained by using 0.1 equivalent nolmal solutions of the different salts and by employing $15 \mathrm{~g}$. of the same kind of charcoal (Merck's blood charcoal) per 100 ec. of solution. The figures represent the per cent of the original salt content removed from solution by adsorption. ${ }^{4}$

\section{The exceptional position of the $\mathrm{H}$ - and $\mathrm{OH}$-ions}

At this juncture also our attention is especially attracted to the $\mathrm{H}$ - and OH-ions; they assume an exceptional position and belong to the most strongly adsorbable ions. Only the ions of the "noblest" metals, a few polyvalent $\left(\mathrm{Fe}^{+++}\right)$and some organic ions rival the $\mathrm{H}$-ions, and only the most easily adsorbed acid dye ions compare with the $\mathrm{OH}$-ions. In this connection an analogy to the chemical combinations among ions suggests itself. In aqueous solutions most ions combine among themselves but to a very slight extent, i.e., most salts are "strong" highly dissociated electrolytes. Only among the acids and bases are there countless weak electrolytes. This means that the H- and $\mathrm{OH}-$ ions bind other ions more firmly than all other ions. In an exactly similar way, the $\mathrm{H}$-ion is more firmly bound by charcoal than the other cations and $\mathrm{OH}$-ion more firmly bound than the other anions. To be sure, it is impossible to effect the

${ }^{3}$ Hans Hartleben, Bioch. Zeitschr. 115, 46 (1921).

4 The figures in the original article were recalculated in the following manner. From the given data of the rough analyses a correction for the water content of the charcoal ( 30 per cent) was applied. Furthermore, all concentrations were recalculated into terms of normal equivalents, for some of the original data were given in terms of molarity. 
adsorption of $\mathrm{H}$-ions alone by charcoal, the entire acid molecule $\left(\mathrm{HCl}, \mathrm{HNO}_{3}\right.$, ctc. $)$, being adsorbed. The adsorption of the H-ions cannot proceed to their specific adsorption equilibrium, for they are held back in solution by the much less adsorbable Cl-ions (or the corresponding anions of the acid). On the other hand, more Cl-ions are adsorbed than would correspond to their specific adsorption equilibrium since some are carried along by the better adsorbable H-ions. The actual adsorption equilibrium represents an average somewhere between the specific equilibria of the two ions (i.e., the $\mathrm{H}$-ion and the anion). It is of obvious interest to determine the adsolption of the $\mathrm{H}$ - and $\mathrm{OH}$-ions alone. In order to achieve this end the experiment must be so arranged that the establishment of equiliblium be hindered as little as possible by opposing forces. This can be approximately accomplished by studying the adsorption of $\mathrm{HCl}$ in presence of a large excess of $\mathrm{KCl}$. The reason for this is the following:

Let there be given a chemical system consisting of a dilute aqueous solution of a non-electrolyte (e.g., acetone) and of a solid adsorbent, such as charcoal. The adsorption equilibrium in this system is attained. Further, let the extension of the phases be so great, that the addition to or the removal from the solution of one mol of acetone would cause no appreciable change in concentration.

Under these conditions the equilibrium attained is characterized by the fact that no work is required to transfer a relatively very small amount, e.g., $1 \mathrm{~mol}$, of acetone from the charcoal surface into the solution. But if we assume that no state of equilibrium has been attained, and that the concentration of acetone is smaller in the solution than it is on the charcoal surface, then there should be present a tendency to reach equilibrium. ' If we could render this process reversible, we should be able to gain a certain amount of work. In order to calculate the amount of this work let us imagine the process of attaining equilibrium as occurring in two stages. In the first we shall compress the solution, by means of an osmotic piston, until it has that concentration which wou'd be in equilibrium with the charcoal surface, thus changing the concentration from $\mathrm{c}$ to $\mathrm{c}_{0}$. To obtain this result we must expend an amount of work $n \mathrm{RT} \ln \frac{\mathrm{c}_{0}}{\mathrm{c}}$, where $\mathrm{n}$ is the number of mols of acetone in the solution. Now let $1 \mathrm{~mol}$ of acetone pass from the charcoal surface into the solution. 
Since this will occur in the state of equilibrium, no work will be performed. Finally, we shall dilute our solution, again by means of the osmotic piston, to its original concentration, whereby the amount of work, $(\mathrm{n}+1) \mathrm{RT} \ln \mathrm{c}_{0}$, will be gained, since there are now $\mathbf{n}+\mathbf{1}$ mols of acetone in the solution. The algebraic sum of the work expended and gained gives the amount of work gained, RT $\ln \frac{\mathrm{c}_{0}}{\mathrm{c}}$. Thus the amount of work depends upon the ratio of the given concentration to the concentration at equilibrium.

When an electrolyte, such as $\mathrm{HCl}$, is adsorbed, a similar process takes place. The easily adsorbable $\mathrm{H}$-ion must carry along with it the less easily adsorbable Cl-ion, so that more of $\mathrm{Cl}^{-}$is adsorbed than would correspond to the true $\mathrm{Cl}^{-}$adsorption equilibrium. To accomplish this a certain amount of work must be applied, which depends upon the ratio of the given $\mathrm{Cl}^{-}$concentration of the solution to that $\mathrm{Cl}^{-}$concentration which would correspond to the specific $\mathrm{Cl}^{-}$adsorption equilibrium. When a definite amount of $\mathrm{Cl}^{-}$had been carried along upon the charcoal surface, then the value of the ratio of the original $\mathrm{Cl}^{-}$concentration to the final concentration approaches the closer to 1 the greater the $\mathrm{Cl}^{-}$concentration. Therefore, the H-ions can carry along the necessary Cl-ions out of a solution rich in $\mathrm{Cl}^{-}$almost without an expenditure of energy, and the true adsorption equilibrium of $\mathrm{H}$-ions is thus established. If this theory is correct, the adsorption of $\mathrm{HCl}$ should be increased through the addition of $\mathrm{KCl}$. This has been experimentally confirmed. ${ }^{5}$ This result was not one to be expected as a matter of course. For hitherto it had always been observed that the presence of another substance decreased the adsorption of the first substance ("adsorptiondisplacement" ${ }^{6}$ while in this instance the very opposite was the case. By constantly increasing the concentration of the added $\mathrm{KCl}$ in a $0.01 N \mathrm{HCl}$ solution a limiting value for the adsorption of the $\mathrm{HCl}$ is reached which represents the true adsorption equilibrium of the $\mathrm{H}$-ions. The theory was confirmed by finding that the adsorption of $\mathrm{HNO}_{3}$ when accompanied by an increasing concentration of $\mathrm{KNO}_{3}$ led to the same limiting value for $\mathrm{H}^{+}$-adsorption. In the same

- Rona and Michaelis, Bioch. Zeitschr. 97, 85 (1919).

${ }^{6}$ Michaelis and Rona, Bioch. Zeitschr. 15, 196 (1908); 16, 489 (1909); Masius, Über die Adsorption in Gemischen. Diss. Leipzig 1908; Freundlich and Masius, Gedenkboek van Bemmelen 1910. 
way it was possible to estimate the true adsorption equilibrium of $\mathrm{OH}$-ions by the addition of $\mathrm{NaCl}$ to a $\mathrm{NaOH}$ solution. The striking result was in the finding that the adsorbability of $H$-ions and the $O H$-ions is practically the same.

The full value of the experiment in demonstrating quantitatively the above relations is obtained only, when it is possible to assume that the $\mathrm{K}$-ions are so poorly adsorbed in comparison with the $\mathrm{H}$-ions, that they do not increase the adsorption of the H-ions, in spite of their greater concentration. This assumption is practically quite justifiable, for the very slight degree of adsorption of K-ions in comparison with $\mathrm{H}$-ions is easily demonstrable by separate adsorption tests with pure $\mathrm{HCl}$ and $\mathrm{KCl}$ solutions. The above does not hold true for acids with strongly adsorbable organic anions. Thus, for instance, salicylic acid is adsorbed to a much greater extent than $\mathrm{HCl},{ }^{7}$ apparently because its organic anion is more strongly adsorbed than its $\mathrm{H}$-ion.

In this way the following figures were obtained. The limiting value of the adsorption of $\mathrm{HCl}, \mathrm{HNO}_{3}, \mathrm{H}_{2} \mathrm{SO}_{4}, \mathrm{FOH}$ and $\mathrm{NaOH}$, in the presence of an excess of the corresponding salts, from a $0.01 \mathrm{~N}$ solution by one per cent by weight of the same kind of charcoal (Merck's blood charcoal) was 40-45 per cent. (From a $0.01 \mathrm{~N} \mathrm{NaCl}$ solution, under the same conditions, the limiting values were 2 to 5 per cent).

\section{Charcoal as an insoluble ampholyte : acidoid, basoid, ampholy- toid, saloid}

An acid has been defined above (see p. 18) as a molecular species which, while maintaining its negative electric charge, binds $\mathrm{OH}-\mathrm{ions}$, and a base as one binding H-ions. This definition could also be extended to substances which are not molecularly dispersed. Thus we could say that charcoal is an absolutely insoluble ampholyte, not molecularly dispersed, which binds $H$ - and $O H$-ions to the same extent. Its isoelectric point is, therefore, in the vicinity of the true neutral reaction. In the section on electroendosmosis it will be shown how closely this theory agrees with the observations made upon the electric charge on charcoal.

An attempt to apply the concepts of acid, base and ampholyte to an absolutely insoluble substance may, however, lead to a certain

7 Unpublished results. 
confusion. For this concept of acid, base, etc., is bound up with the property of electric conductance in aqueous solutions. This property, to be sure, does not entirely disappear in our non-molecularly dispersed substances, and we shall shortly recognize its share in conductance phenomena under the form of electrophoresis. But in order to avoid any possible confusion it would be better to restrict the use of the terms "acid, base, ampholyte, salt" for the water-soluble, true electrolytes, and the non-molecularly dispersed substances which, because of their capacity to adsorb OH-ions, are analogous to acids will be designated as acidoids. The following definitions are given:

An acidoid is a non-molecularly dispersed substance which adsorbs $\mathrm{OH}$-ions (or dissociates off $\mathrm{H}$-ions) while maintaining a negative charge (or adsorbs $\mathrm{H}$-ions on neutralization of its negative charge). A basoid is analogously defined, and charcoal is a good example of an ampholytoid. Between a true acid (or base, or ampholyte) and a coarsely dispersed acidoid (or basoid, or ampholytoid) the colloidal substances form a multitude of intermediate stages.

In adsorbing a dissolved base an acidoid forms a salt-like surface compound which we shall designate as a saloid. Charcoal, being an ampholytoid, forms saloicls, with acids as well as with bases. In addition, behaving like a true ampholyte, charcoal forms with $\mathrm{KCl}$ a double saloid, just as an amino acid, at least in its solid state, forms a double salt compound with $\mathrm{KCl}$. Also, quite analogously, the tendency of charcoal to combine with $\mathrm{HCl}$ or $\mathrm{NaOH}$ is much greater than that of combining with $\mathrm{KCl}$, just as in the case of an amino acid.

The author is aware of the fact that the consideration of the socalled "chemically unreactive" substances and of ampholytes under a common heading will not meet with universal acceptance, and that the phenomenon of adsorption will be regarded for a long time to come in the light of a process quite distinct from those of ordinary chemical reactions. In the age of the atomic model and of the reduction of chemical affinity and of the forces of cohesion to a common basis of electrostatic forces, it would appear quite appropriate to do away with such limitations. Henceforth, then, in speaking of adsorption processes, it should be clearly understood that they do not represent a fundamental contrast to chemical union. Since we can only speak of adsorption when the reaction is limited to the boundary sur- 
face of two adjacent phases, the isolation of the resulting compound in a pure state is not possible, and, therefore, its stoichometric relations cannot as yet be established.

At this juncture we must return to the extended definition of adsorption given on page 23t. When the substance of the nonaqueous phase does not display a marked molecular attraction either for, $\mathrm{H}$ - or $\mathrm{OH}$-ions, then it behaves towards a solution containing $\mathrm{H}^{+}$- or $\mathrm{OH}^{-}$-ions exactly as a mass-free space, and in such a case the accumulation of the $\mathrm{H}-$ or $\mathrm{OH}-i o n s$ at the surface must follow simply in accord with Gibbs' principle. It is not possible to predict whether the $\mathrm{H}$ - or $\mathrm{OH}$-ions will show more surface-activity. But experience tells us that numerous non-aqueous phases which are chemically neither acids nor bases assume a negative charge when in contact with pure water. This is a partial aspect of Coehn's law (see below, page 28S). The common basis of all these phenomena renders it probable that the non-aqueous phase does not participate in the $\mathrm{H}^{+}-\mathrm{OH}^{-}$-distribution, and that this distribution occurs as if the aqueous phase were in contact with an empty space. The negative charge on the non-aqueous phase in respect to the aqueous phase leads one to postulate that the $\mathrm{OH}$-ions are more strongly sulface-active than the $\mathrm{H}$-ions, that, therefore, the $\mathrm{OH}$-ions accumulate at the surface of the water phase, and that consequently the interior of the water phase becomes positive in respect to its own surface layer and hence also in respect to the adjacent phase. If we are to apply here our extended definition of adsorption, we must suppose that such chemically indifferent substances, as ethyl ester, benzonitrile, oils, hydrocarbons, etc., have a greater tendency to adsorb OH-ions than $\mathrm{H}$-ions, and are, therefore, to be considered acidoids. But even substances not chemically indifferent are more frequently negatively charged towards water rather than positively. Thus Freundlich and Gyemant $^{8}$ have found that aniline is negatively charged towards water or towards water saturated with aniline. It could perhaps be expected that aniline, being a base, must adsorb $\mathrm{H}$-ions $\left(\mathrm{C}_{6} \mathrm{H}_{5} \mathrm{NH}_{2}\right.$ $+\mathrm{H}^{+}=\mathrm{C}_{6} \mathrm{H}_{5} \mathrm{NH}_{3}{ }^{+}$), and that at all events it should adsorb H-ions much better than $\mathrm{OH}$-ions. But this is quite erroneous. 'Those few aniline molecules which have combined with H-ions have become water-soluble aniline-ions, and we could scarcely expect any signifi-

${ }^{8}$ II. Freundlich and A. Gyemant, Zeitschr. f. physikal. Chem. 100, 182 (1922). 
cant amount of such would remain in the boundary layer. Otherwise aniline behaves as a chemically indifferent substance, and there is nothing in the way of $\mathrm{OH}$-ions accumulating in the surface layer in accord with Gibbs' theory. It could then be stated that aniline not dissolved in water is of the character of a very weak acidoid. No matter what nomenclature is adopted, instances will be met with which are not adequately provided for. Such cases are disposed of by our "extended" definitions. The most exact of the sciences, mathematics, furnishes ample illustrations of this. Thus the definition: " $a-b$ is the sum of the terms $+a$ and $-b$ " is an instructive example. For, according to the original definition of a sum, $a-b$ is no sum at all, but its exact opposite, a difference. Likewise, we shall have to accept the idea, that aniline may either be a base or an acidoid, according to the conditions and to the point of view.

Whether or not the acidoicl- or basoid-nature of a substancevaries as its true chemical acidic or basic character, depends upon the relative intensity of the acidic or basic character. The very weak base aniline is thus an acidoid, while the much stronger base $\mathrm{Al}(\mathrm{OH})_{3}$ is a basoid in respect to pure water. Hence only substances with a very pronounced basic character can behave as basoids, and that is why basoids occur relatively much more rarely than acidoids. And most of these are basoids only towards a neutral or acid solution, for most of them chemically are ampholytoids possessing an isoelectric point, as, for example, $\mathrm{Al}_{2} \mathrm{O}_{3}, \mathrm{Fe}_{2} \mathrm{O}_{3}, \mathrm{ZnO}$, and other similar substances.

\section{The equivalent adsorption of ions of a salt by charcoal; the neutralization effect of charcoal}

The property of the H-ions of being more strongly adsorbed by charcoal than the other cations leads to the following consequence. A solution containing any number of ionic species and whose reaction is acid never becomes more acid in reaction on being shaken with charcoal. On the contrary it becomes less acid. An alkaline solution behaves in a reverse manner under these conditions, becoming less alkaline. ${ }^{9}$ Thus, as a matter of rule, any solution on being treated with charcoal approaches neutrality, and the reaction of a neutral solution never becomes markedly acid or alkaline on treatment with charcoal.

${ }^{9}$ W. Löffler and K. Spiro, Helvet. chim. Acta 2, 417 (1918) and 2, 533 (1919). 
Exactly in the same way the reaction of any aqueous solution may be made to approach neutrality by the addition of a true ampholyte whose isoelectric point lies in the vicinity of neutrality. Hence it follows that in an aqueous solution of a neutral salt, or even of a salt of an organic dye, equivalent amounts of anions and cations are adsorbed, i.e., the salt molecule is adsorbed as a whole. Michaelis and Rona ${ }^{10}$ designated this phenomenon as the equivalent ionic adsorption of salts, and established the fact that no hydrolytic cleavage of salts takes place in the process of adsorption on charcoal. The second observation stated above, to the effect that an acid or alkaline solution approaches neutrality on treatment with charcoal could have been deduced from these observations of Michaelis and Rona, but was not recognized by them at the time. It was found experimentally by Löffler and Spiro, ${ }^{9}$ and this phenomenon ean be designated as the neutralization effect of charcoal. ${ }^{10 a}$

\section{Other adsorbents}

Charcoal possesses the peculiarity of not itself yielding any ions to a solution. As soon as an adsorbent contains ionogenic radicals, it must be taken into account that the adsorbent itself may show a

${ }^{10}$ Michaelis and Rona, Bioch. Zeitschr. 94, 225; 97, 57; 102, 268 (1920).

10s It developed later that the above described amphylotoid nature of charcoal is not a general property but is rather peculiar to certain varieties or preparations of charcoal. It seems to have little to do with the mineral impurities, but to depend much more upon the organic material from which the charcoal is prepared, and above all upon the temperature at which the carbonization is carried out. It now appears that with rising temperature of charring all charcoals tend to reach a single definite limiting state. This fact was first brought out by Miller and Bartell (Jour. Amer. Chem. Soc., 44, 1866 (1922)) with highly heated (activated) ash free charcoal from sugar. This charcoal is not of ampholytoid character. For it adsorbs $\mathrm{HCl}$ but to a slight extent, does not adsorb $\mathrm{NaOH}$ at all, while neutral salts are adsorbed by it in such a manner that a trace of acid is adsorbed, leaving a slightly alkaline solution, the adsorption being thus a hydrolytic one.

On the other hand blood charcoal which had not been activated by thorough heating will yield all the adsorption phenomena described in this section (I. Ogawa, Biochem. Zeitschr., 172, 249 (1926)). No recognizable differences were observed in the elementary analyses of the different charcoal preparations before and after activation, making it probal,le that the changes occur only in the ultimate crystal structure of the substance, in spite of the fact that the x-ray diagrams obtained by the method of Debye and Scherrer show no differences between the two states. 
tendency to form ions or to exchange ions with the solution about it. The simplest case is that of an adsorbent of the type of an insoluble acid (silicic acid, mastic-resin acid), or of an insoluble base (metal oxides). It was found that in such cases the adsorption proceeds along the lines of ordinary chemical laws, and here it would be quite futile to attempt to demonstrate any contrast between chemical reactions and adsorptions. Thus, for example, ferric oxide (colloidal ferric hydroxide) adsorbs picric acid and eosin, because ferric picrate and ferric eosinate are insoluble. Likewise, silicic acid adsorbs methylene blue, because methylene blue-silicate is insoluble. To be sure, the experimental study of this state is beset with unexpected difficulties. For it is almost impossible to obtain such "insoluble acids or bases" free of ions adsorbed on their surfaces with which they form insoluble salts. Thus there is no silicic acid preparation free of $\mathrm{Ca}^{++}, 11$ and there is no colloidal iron preparation free of $\mathrm{Cl}^{-}$(or of some other anion, depending upon the method of preparation) with which it forms an insoluble basic salt. For this reason those ionogenic adsorbents which may be conceived of as insoluble "salts" are more convenient for experimental work. These are represented by such substances as kaolin (silicates of alkaline earths) and the socalled ferric oxide (basic ferric chloride). Such adsorbents behave entirely according to the well known laws of chemical reactions and react with the electrolytes in aqueous solution by means of an exchange of ions. A solution of methylene blue chloride reacts with kaolin according to the following scheme:

Alkaline earth silicate + methylene blue chloride $\rightleftharpoons$ Methylene blue silicate + alkaline earth chloride

Since methylene blue silicate is insoluble, and the alkaline earth chloride is soluble, the kaolin exchanges its alkaline earth cation (especially the ever present $\mathrm{Ca}^{++}$) for the methylene blue cation, while the $\mathrm{Cl}$-ion of the methylene blue remains in solution, not as $\mathrm{HCl}$, however, but as $\mathrm{CaCl}_{2}$. An exchange of ions between kaolin and $\mathrm{NaCl}$ cannot be demonstrated by analytical methods, although doubtlessly according to general chemical laws such an exchange can take place. Not a trace of eosin or picric acid is adsorbed by kaolin, for an exchange of ions in these instances would not result in the formation of insoluble salts.

${ }^{11}$ Michaelis and Rona, Bioch. Zeitschr. 97, 57 (1919), cf. p. 71. 
It would be of great interest to study the adsorption capacity of an indifferent substance, such as cellulose (filter paper, cotton wool), but unfortunately great experimental difficulties arise. It is quite impossible to obtain a cellulose entirely free of ash. Even after the most careful extraction with hydrochloric and hydrofluoric acids it always contains some silica and lime. In studying the adsorption of dyes on cellulose, Michaelis and Rona ${ }^{12}$ found invariably adsorption by exchange of ions. Thus after the adsorption of methylene blue chloride the cation was found to have been adsorbed while the anion $\left(\mathrm{Cl}^{-}\right)$was found in solution as a neutral salt $\left(\mathrm{CaCl}_{2}\right)$. Similarly, in the adsorption of $\mathrm{NH}_{4}$ - eosin, the eosin anion was adsorbed, while at least 70 per cent of the $\mathrm{NH}_{4}$ - ion remained in solution as a neutral salt. Since "adsorption by exchange" exercises so great an effect it has been practically impossible to ascertain the extent to which the cellulose itself participates in the adsorption process. It must be, in any case to an extremely minute extent. The ability of the technical forms of cellulose (cotton, paper etc.) to take dyes must depend largely upon an exchange of ions with its ash constituents, and the latter form quite possibly an integral part of the cellulose molecule. The properties of cellulose are very different from those of charcoal, in which, to be sure, there is also no lack of these impurities, but in which the much greater adsorbing capacity of the charcoal itself renders any effect due to the impurities negligible.

It is not the purpose of this book to exhaust the problem of ionic adsorption. The discussion offered above should only serve as an introduction to the subject of adsorption potentials, and it should suffice for this purpose.

\section{The formation of a charge upon phase boundary surfaces due to adsorption of ions}

In the light of the explanations given in the preceding section we can ascribe to each kind of ion its own specific adsorbability. But since the electrostatic forces prevent the adsorption of the two ions of an electrolyte to measurably different extents, what really happens is the establishment of an adsorption equilibrium which lies somewhere between the hypothetic equilibria of the positively and

12 Michaelis and Rona, Bioch. Zeitschr. 103, 19 (1920). 
of the negatively charged ions. But at the same time a potential difference is formed at the phase boundary surface. Still the more adsorbable of the two ions is adsorbed to a greater extent than the other ion, this difference being demonstrable, if not by analysis, then by some other means. Charcoal in contact with dilute $\mathrm{HCl}$ solution adsorbs somewhat more $\mathrm{H}$-ions than $\mathrm{Cl}$-ions. While the difference cannot be demonstrated by analysis, it becomes evident from the fact that the charcoal surface has become positively charged towards the solution. The adsorption of the H-ions in excess proceeds to such an extent that the potential due to the separation of the ions just compensates the repulsion tendency of the ions themselves. In this way can we explain the long known fact that a potential difference arises at the boundary surface of any two different phases in general. As the means of studying and recognizing this formation of electric charges we have at our disposal two apparently very different, and intrinsically quite correlated, methods: electric endosmosis (electroendosmosis) and electric cataphoresis (electrophoresis).

In entering now upon the discussion of the so-called electrokinetic (Helmholtz) or electroosmotic (von Smoluchowski) phenomena, we shall for the time being apparently lose our connecting thread with the preceding chapter, but eventually we shall reëstablish the connection.

\section{The earlier history of electroendosmosis}

In 1808 Reuss $^{13}$ made the following observation: If two tubes filled with water are partly immersed in wet clay, and a galvanic current is then sent through them by means of metal electrodes, the water rises to a higher level in the tube on the cathode side and falls to a lower level on the anode side. This was the discovery of electroendosmosis. At the same time particles of the clay become loose and travel towards the anode, as shown by the clouding of the water in the tube. This was the discovery of electrophoresis. Both phenomena are, as it were, the expression of the positive charge of the water with respect to the clay. If the clay is thought of as a solid filter or diaphragm for the water, then only the movement of

${ }^{13}$ F. Reuss, Mém. de la Soc. imp. des Naturalistes de Moscou. 2, 327 (1809). 
the positively charged water towards the cathode would be possible. But the clay particles which are freely movable in the water also exhibit their negative charge by their motion towards the anode. The meaning of the endosmosis was not clear, and for a time little attention was paid to it. It was again described in 1816 for sand filters by Porret, ${ }^{14}$ and then also by Becquerel, Armstrong, and Daniell. The phenomenon of electrophoresis was further described in tissue fibers by Faraday, ${ }^{15}$ in starch grains by DuBois Reymond, ${ }^{16}$ and in carmin and starch by Heidenhain and Jürgensen. ${ }^{17}$ The first more exact investigations on electroendosmosis were carried out by Wiedemann, ${ }^{18}$ and the first theoretical treatment of this subject approaching the views held today, was furnished by Quincke. ${ }^{19}$ This modern theoretical conception resulted from the observation that water does not always move in the direction of the current but at times moves against the current. In this way Quincke showed that the original idea to the effect that water "was carried along by the current" was not correct. He then proposed the theory of the electric double layer which has since proved to be so fruitful. Quincke assumed that at the boundary of fluids with solids two layers of free electricity, one positive and one negative are found at a very small but finite distance from each other. The one forms a coating adhering fast to the solid, while the other is in the fluid medium. The latter layer can be parallelly displaced along the former by means of a tangentially directed current of electric forces. If the solid be a particle suspended in the fluid, then this displacement is manifested in the motion of the particle under the influence of an electric field. If the solid is a porous solid in the nature of a diaphragm blocking the path of the water, then, in an electric field, the water will migrate through the pores of the diaphragm in the opposite direction.

The quantitiative relationships of this theory of these phenomena were developed by Helmholtz. ${ }^{20}$

${ }_{14}$ R. Porret, Gilb. Ann. 66, 272 (1816).

15 M. Faraday, Exp. Res. Nr. 1562, 1838.

${ }^{16}$ E. Du Bois-Reymond, Berl. Ber. 1860, p. 895.

${ }^{17}$ E. Heidenhain u. Jürgensen, Arch. f. Anat. u. Physiol. 1860, p. 573.

18 Wiedemann, Ann. d. Phys. u. Chem. 87, 321 (1852).

${ }_{19}$ Quincke, Ann. d. Phys. u. Chem. 113, 513 (1861).

${ }^{20}$ H. v. Helmholtz, Ann. d. Phys. u. Chem. 7, 337 (1879); Ges. Abhandlungen I, p. 855 (1882). 


\section{Helmholtz's theory of electroendosmosis ${ }^{21}$}

Let us imagine that a solid diaphragm with capillary pores is inserted into a tube filled with aqueous fluid. Now, on sending an electric current through this tube, we observe that the water is set into motion, and that after a short time a flow of water with a constant velocity will ensue, provided care is taken to prevent hydrostatic difference of pressure due to the migration of the water. Let us now consider this stationary condition of our system. To begin with, it is characterized by the fact that the motion produced by the electric current is rendered uniform by the friction. Now the electric force is proportional to the electric density of the double layer $\sigma$, and to the electric field intensity, $\mathrm{H}$, to which the double layer is exposed. In terms of absolute units the electric force is simply equal to $\sigma \mathrm{H}$. This value remains constant throughout the entire process. If no friction were present it would produce a uniformly accelerated motion of the water. But the friction, checking the motion, changes its rate from a uniformly accelerated to a uniform one. The force of friction is proportional to the specific friction coefficient of water, $\eta$ to the velocity $\mathrm{v}$, and inversely proportional to the distance between

${ }^{21}$ The theory is not expounded here in Helmholtz's original terms and scope, for that would require of the reader an extensive knowledge of theoretical physics. It is presented in its simplified form, as given by Perrin. Helmholtz's own presentation has the advantage of greater strength, especially in regard to the absolute value of the proportionality factors, which is not so convincing in Perrin's interpretation. But since the assumption on which Helmholtz's calculations are based have perhaps not been strictly justified (cf. section 78), and since the experimental data available thus far is still insufficient to confirm the theory unqualifiedly, the mode of interpretation given here will have to suffice, in a preliminary way, for physiologists.

The difficulty in the way of experimental quantitative confirmation of the theory is the following: Adsorption potentials cannot yet be recognized in any other way except through electrokinetic phenomena (electrophoresis, electroendosmosis, hydrodynamic currents and currents of falling particles). Thus, for example, in Helmholtz's theory the wall potential is calculated from the figures given by electroendosmosis. The theory could only be confirmed by comparing the value of the potential obtained from electrokinetic data with that obtained by using some other method which is entirely independent of electrokinetic technic. Such a method is still unavailable. And thus the quantitative aspect of the theory is still uncertain, while in its qualitative aspect is of great service. 
the layer set in motion and the wall. Hence, in our stationary condition we have

$$
\sigma \mathrm{H}=\eta \times \frac{\mathrm{v}}{\mathrm{d}}
$$

where $d$ is the distance between the moving electric layer and the resting layer, hence it is the thickness of the double layer.

If, on the other hand, the volume $\varphi$ of the fluid displaced per unit of time is measured, then the velocity $\mathrm{v}$ can be expressed in the following terms, on the assumption that the diaphragm is represented by a single capillary whose average cross section has the radius $\mathrm{r}$ :

$$
\pi \mathrm{r}^{2} \mathrm{v}=\varphi
$$

from which it follows that

$$
\sigma \mathrm{d}=\frac{\eta}{\pi \mathrm{r}^{2}} \times \frac{\varphi}{\mathrm{H}}
$$

This equation permits us to calculate the potential $\zeta$ at the phase boundary surface from the velocity of the water transport. The potential difference between the moving and the resting layer is, according to the laws of theoretical physics,

$$
\zeta=\frac{1}{\mathrm{~K}} \times 4 \pi \sigma \mathrm{d}
$$

K represents here the dielectric constant of water (Helmholtz left this value out of consideration, and it was first applied by Pellat and Perrin), therefore,

$$
\zeta=\frac{4 \pi}{\mathrm{K}} \times \frac{\eta}{\pi \mathrm{r}^{2}} \times \frac{\varphi}{\mathrm{H}}
$$

If the diaphragm consists of a large number, $\mathrm{n}$, of similar capillaries of radius $r$, the total cross section of all of them being $s$, and the amount of water transferred in a unit of time being $\Phi$, then correspondingly:

$$
\zeta=\frac{4 \pi}{\mathrm{K}} \times \frac{\eta}{\mathrm{s}} \times \frac{\Phi}{\mathrm{H}}
$$

It follows, therefore, that the transfer of the water is proportional to the intensity of the electric field and to the cross section of the diaphragm, but that it is independent of the length of the capil- 
laries, i.e., of the thickness of the diaphragm, as long as the intensity of the field (or the fall of potential per sq. cm.) in the diaphragm remains constant. In other words: The amount of water transported through a diaphragm is proportional to the total current intensity and is independent of the length and of the width of the pores of the diaphragm. It is dependent, however, upon the nature of the substance of the diaphragm as well as of the liquid used. These are exactly the laws which Wiedemann (l. c.) had previously established experimentally.

In the above discussion the assumption was made that the adhering layer is completely motionless. If this were not exactly true, then the value of $\zeta$ as calculated from the above equation becomes somewhat too great. Further, it is also assumed that the dielectric constant $\mathrm{K}$ is the same in the boundary layer of the water as the usual dielectric constant of water. Furthermore, the friction coefficient, $\eta$, must only be applied when it is assumed that the distance between the double layers is indeed small, and yet quite great in relation to the size of a molecule. Lamb ${ }^{22}$ developed a theory in which he neglected these assumptions. But since formally a quite similar equation is derived and only the absolute value of $\zeta$ is affected by it, we shall limit ourselves merely to this mention.

Helmholtz's theory also demands that the diaphragm should consist of only similar and lengthwise parallel capillaries. Smoluchowski extended the theory to diaphragms with capillaries running in any desired direction and found no demonstrable deviations from Helmholtz's laws.

The entire phenomenon of electroendosmosis can be pictured as follows: A thin layer of water lying adjacent to the walls of the pores of the diaphragm is displaced tangentially along these walls by the field of force of the electric current; and this layer carries along with it, because of cohesion, water layers further removed from the walls of the pores. The water is to a certain extent pulled tangentially at its boundary layer.

\section{The theory of electrophoresis}

In a wide U-tube filled with water are suspended fine insoluble (either solid or fluid) particles, and an electric current is sent through the suspension. The current affects, conversely to the preceding case,

${ }^{22}$ H. Lamb, Phil. Mag. 25, 52 (1888). 
the surface layers of the suspended particles, and the particles move in the reverse direction, in relation to the water in the preceding case. This is the phenomenon of electric cataphoresis or electrophoresis. As long as a wide $\mathrm{U}$-tube is used, this phenomenon is merely the reverse of endosmosis. But if this phenomenon is to be studied in narrow tubes, suitable for microscopic observation, certain peculiar complications arise. For, in such narrow tubes, not only do the suspended particles show motion in relation to the water, but the water itself shows relative motion along the walls of the tubes. This is explained by the formation also along these quartz or glass walls of a double layer towards the water, and the water is pulled by its surface layer adjacent to the walls of the tube, under the influence of the external electric tension of the double layer. Therefore, the motion of the suspended particles, as observed on the microscope stage, represents in reality the algebraic sum of cataphoretic motion of the particles in relation to the water and of the motion of the particles induced by the streaming of the water itself as explained above. The theory of this phenomenon was theoretically developed by $\mathrm{R}$. Ellis $^{23}$ and was further perfected in a very beautiful manner by Smoluchowski. ${ }^{24}$ Only the results of these investigations will be discussed here.

The theory in its simplest terms applies to a narrow chamber or vessel which is closed on all sides, and from which the water cannot flow out when in motion. This may be represented by such a vessel as a microscopic counting chamber into which the electrodes are sealed in hermetically through two sides. The current of water arising at the outer layers adjacent to the chamber walls must result in a backflow in the interior layers of the water. Therefore the rate of motion of the water is not a constant value, but its value as well as its direction depend upon the distance of the water-particles involved from the walls of the chamber. Let us further assume that in so narrow a chamber only lateral (lamellar) streaming of water is possible, i.e., that every water-particle can move only from left to right or reversely, but not forward and backward, nor up and down. Then the backflow of the water manifests itself only in left-and-right streaming free from whirls. If the water has a certain velocity in the immediate

${ }^{23}$ Risdale Ellis, Zeitschr. f. physikal. Chem. 78, 321 (1911).

${ }^{24}$ M. v. Smoluchowski, Elektrische Endosmose und Strömungsströme, in Graetz' Handbuch der Elektrizität, Bd. II, Leipzig 1921. 
vicinity of the upper or lower chamber wall, then the velocity of the interior layers must rapidly decrease, become zero, and then reverse itself. The velocity $\mathrm{v}$ is hence a function of the distance $\mathrm{x}$ of the water lamella or layer from the chamber wall, or simpler, it is a function of the "depth." If the total depth of the chamber is d, than this function must become symmetrically arranged at one half of the depth of the chamber, where $\mathrm{x}=\mathrm{d} / 2$.

Therefore, the observed motion of a particle depends upon the value of $x$, and it is the algebraic sum of its own cataphoretic motion and of the streaming of the water occurring at the distance $x$. Hence the true velocity of cataphoresis is observed only at that depth $\mathrm{x}$ at which the water streaming $=0$, and this, according to Smoluchowski, is the case for both layers at

$$
\mathrm{x}=\mathrm{d}\left(\frac{1}{2} \pm \frac{1}{\sqrt{12}}\right)
$$

or

$$
\mathrm{x}=\text { approximately } 0.2 \mathrm{~d} \text { and } 0,8 \mathrm{~d}
$$

It is to be noted that the depth $d$ of the chamber may have any value, provided it remains quite small, so that only lamellar currents without whirls are possible. ${ }^{25}$ In macroscopic transference apparatus this condition does not prevail, and in these the streaming of the water plays no important rôle.

If the true velocity of electrophoresis is ascertained in this way, then, on the basis of Helmholtz's assumptions and Smoluchowski's development of Helmholtz's theory, we can calculate the wall potential $\zeta$, and hence also the potential difference between the solution and the surface of the moving particle, from the following equation:

$$
\zeta=\frac{4 \pi \cdot \mathrm{v} \cdot \eta}{\mathrm{K} \cdot \mathrm{H}} \text { or } \mathrm{v}=\frac{\zeta \cdot \mathrm{K} \cdot \mathrm{H}}{4 \pi \cdot \eta}
$$

where $\mathrm{v}$ is the electrophoresis velocity in cm. per second, and $\eta, \mathrm{K}$ and $\mathrm{H}$ have the designations given above. The most important aspect of this equation is that (with constant viscosity) the potential is proportional to the electrophoresis velocity. (If $\zeta$ is expressed in volts, $\mathrm{v}$ in $\mathrm{cm} . / \mathrm{sec}$., $\mathrm{H}$ in volts/cm., then in order to reduce the ab-

${ }^{25}$ The conditions under which this occurs were worked out by O. Reynolds. Phil. Transact. 174, 935 (1883). 
solute units of the potential into volts it is necessary to multiply by the factor $\left.(1 / 200)^{2}\right)$.

It follows from the above that the velocity of the migration is independent of the size of the particles, which agrees with the findings of Burton ${ }^{26}$ in colloidal silver solution containing particles of various dimensions.

We must be warned of an erroneous interpretation of the phenomenon of electrophoresis. Since it is observed that on the application of an external potential the particles suspended in a liquid move towards one of the poles, it might appear as if every particle behaved as a body carrying a free electric charge and was attracted to the oppositely charged pole, just as a charged hair is attracted towards a charged piece of sealing wax. Such an interpretation would be quite incorrect. The suspended particles, considered as a whole, are

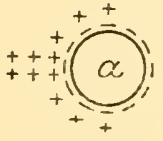

B

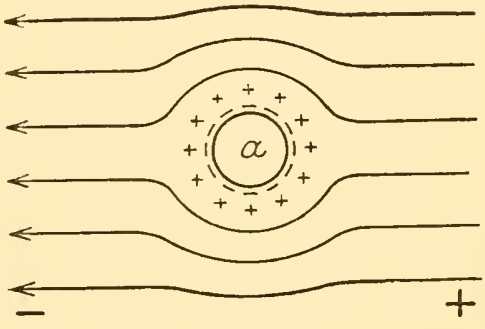

A

Fig. 28

electrically neutral, for the charges on the positive and negative double layer are equal to each other. In a certain respect this attraction might be compared rather to that existing between an electrically charged piece of sealing wax and an uncharged small metal ball. In the latter case it is said that the metal ball becomes charged or polarized under the influence of the electrical field of the sealing wax. But this comparison is still unsuitable, for our metal ball would be attracted equally well by a positive or a negative pole, while the electrophoretically moving particle always migrates in a definite direction away from one pole and towards the other pole. It has no "free" charge which could be detected by bringing the liquid containing the particles near an electroscope. The external field of force of such a suspension or colloidal solution is naturally equal to

${ }^{26}$ E. F. Burton, Phil. Mag. 11, 439 (1906). 
zero. The effect of an externally applied electric field upon such a system is but a tangential, gliding displacement of both of the layers constituting the double layer.

The mechanism of electrophoresis can be depicted as follows. In fig. $28 \mathrm{~A}$ let a be a small ball which is surrounded by an electric double layer consisting of a solidly adhering negative layer and of a displaceable positive layer. The direction of the lines of force of the external electric field is shown by the arrows. Thus the ball with its fastened negative layer must be displaced to the right and the outer positive layer to the left, so that, for a moment, the state shown in B results. The positive layer which has become defective on the right side regenerates at once, even before the state shown in B is reached, with the help of the positive ions meanwhile brought up by the external electric current. And, likewise, the positive ions which were set free at the left end of the outer layer find their electric equivalents in the negative ions brought up by the current, or, they serve to replace the positive ions lost on the way. As it can be readily seen, the entire ball participates in the conduction of the electric current.

\section{The relation of electroendosmosis and electrophoresis to the ionic theory}

So much for the purely physical investigations on this subject. In these, the chemical nature of the solution and diaphragm being assumed as given, the chief problems were to correlate the extent or value of endosmosis or cataphoresis with such physical data as the intensity of the current and electric field, the width of the pores etc., and from the numerical values of endosmosis and cataphoresis, under the given conditions of current, electric field etc., to calculate the potential difference between the solid wall and the liquid. We shall now consider the question of the effect of the chemical composition of the solid wall and of the solution upon the phenomena of endosmosis and cataphoresis as well as upon the above potential. At the same time the question arises as to chemical structure of the electric double layer, a question which was left open by Helmholtz. In harmony with our present day theory, we shall at once conceive the double layer as being made up of ionic layers, just as we assumed the double layers of electrode and interphase boundary potentials to be composed of layers of free ions. At this point we take up again our connecting thread with the theory of ionic adsorption which we temporarily abandoned in section 70 . 
That the magnitude and even the sign of the charge represented by the double layer depend upon the chemical nature of the phases was indeed not completely overlooked by the earlier investigators, but the question could not have been satisfactorily answered before the advent of the ionic theory. With the development of modern physical chemistry this problem attracted much attention, and we are indebted chiefly to the work of Perrin for the description and interpretation of the fundamental laws underlying these relationships.

The basic idea of correlating all of these phenomena with the ionic theory originated with Nernst, and the conception is really the same as that which we applied for the explanation of phase boundary potentials. Let us imagine that both of our adjacent phases contain ions. Distribution equilibrium is not reached, for the tendency towards equilibrium, because of opposing electrostatic forces, results in the appearance of a potential difference instead of equilibrium. Thus the two strata of the double layer consist of free ions; Helmholtz did not leave us a material conception of the nature of the electric particles of the double layer. At present we can say that they consist either of electrons or of ions. The possibility that electrons alone constitute at least one of the two layers must be admitted in the case where one of the phases is a metal. For the time being we shall deal only with double layers consisting of free ions, and thus the phenomena of endosmosis and cataphoresis become a part of our present discussion of "Ions as Sources of Electric Potential Differences."

This renders the phenomenon of motion in electrophoresis entirely comprehensible. But in addition the following assumption must be made. The point of attack of the forces of the electric field is the motile ionic layer, which in its motion carries along the water. Earlier in our discussion of the conductivity of dissolved electrolytes we have seen that the ions cannot move in water without carrying along with them large numbers of water molecules. It is this strong attraction between ions and water molecules which is responsible also in this case for the fact that the displacement of an ionic layer becomes externally apparent through the displacement of a water layer.

It is pertinent to draw an analogy between the carrying along of water molecules by ions in the simple conductance of an electric current by an electrolyte in solution and that occurring in the process of electroendosmosis, or, to conceive of the common electrolytic 
conductance as of an electrophoresis of suspended particles of molecular dimensions. Let us take again a U-tube, open at both ends, filled with a solution of an electrolyte, and with an electric current passing through it. The anions in moving towards one end of the tube will carry along with them a certain amount of water, while the cations moving to the other end will carry along with them a certain, but different, amount of water. The water should, therefore, rise at one end. But since hydrostatic differences of pressure cannot be maintained, the water pulled upward at every moment by electric forces must immediately sink downward again because of gravity. Therefore the only recognizable manifestation of the varying capacity of ions to bind water is the varying motility of the ions and the changes of concentration resulting from it in the electrolyte solution, which is numerically expressed as the transport number of the electrolyte. In electroendosmosis experiments the presence of the diaphragm permits, under favorable conditions, of the formation of hydrostatic pressure differences, and the displacement of the water can thus be directly observed. Hence, from the purely qualitative standpoint the analogy between both phenomena is quite complete, and Smoluchowski drew our attention to it. Whether this theory is also acceptable on a quantitative basis is not yet entirely clear.

In fact, it will be shown in section 78 that under appropriate conditions, namely, through the introduction of a diaphragm with narrow pores, and whose walls have nopotential towards the solution, it is possible to demonstrate experimentally the displacement of water in an electrolyte solution by the passage of an electric current.

The first purposeful investigations which rendered possible the correlation of ionic adsorption and of endosmosis were made by Perrin.

\section{Perrin's experiments}

Perrin $^{27}$ carried out his experiments shown in figure 29. The diaphragm is represented by the glass tube $M$ which is filled with the powdered substance. A and B are the electrodes, and the motion of the water is observed by the change of level in the almost horizontally ascending tube $\mathrm{G}$. The electric field maintained is, as a rule, such that between the electrodes a potential gradient of 10 volts per

${ }^{27}$ Jean Perrin. Jour. chim. phys. 2, 601 (1904), and 3, 50 (1905). 
$\mathrm{cm}$. is obtained. Perrin first of all found that only liquids of pronounced ionizing capacity showed strong electroendosmosis. Liquids with small dielectric constants which are poor conductors and which permit but little dissociation of electrolytes dissolved in them, displayed very weak or no endosmosis. Thus chloroform, petroleum ether, benzene, oil of turpentine, carbon bisulfide showed no endosmosis at all, in contrast to nitrobenzene, acetone, ethyl and methyl alcohols, and above all to water which endosmotically are very active.

The electrolytes dissolved in the water have a great influence upon the extent and direction of the endosmosis. Of particularly great effect are the acids and alkali bases, hence the $\mathrm{H}$ - and the $\mathrm{OH}$-ions. Perrin found a whole series of powdered substances with which the direction of the endosmosis in faintly acidified water was the reverse of that in weakly alkaline water. In the acid solution the water always migrated towards the anode, and towards the cathode in the alkaline solution, which means that the powdered substance was positively charged towards the acid and negatively charged towards the alkaline solution. Such substances were charcoal, sulfur, carborundum, naphthalene, salol, $\mathrm{CrCl}_{3}$, $\mathrm{AgCl}, \mathrm{BaSO}_{4}, \mathrm{ZnO}, \mathrm{CuO}$, gelatin and others. Table 38 shows the results for a few of the substances in a diaphragm of $1.4 \mathrm{~cm}$. diameter, in cubic millimeters of water per minute.

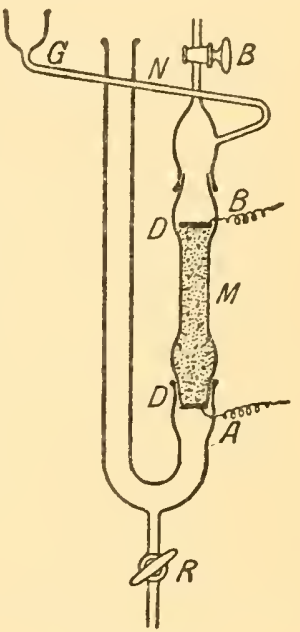

FIG. 29

It is seen from this table that, as a rule, the substances studied hare a positive charge in an acid medium and a negative charge in a basic medium. But the reversal of the sign of the charge is certainly not at true neutrality. Indeed Perrin believed at the time that the cause for this lay in secondary disturbances in the experimental conditions, such as the gradual solution of the powdered substance and the change of reaction resulting therefrom, or the presence of traces of polyvalent ions which, as we shall soon see, have a profound effect upon this process. Perrin deduced the following law:

Every non-metallic substance (in the absence of polyvalent ions) is positively charged in an acid solution and negatively in an alkaline solution. 
TABLE 38

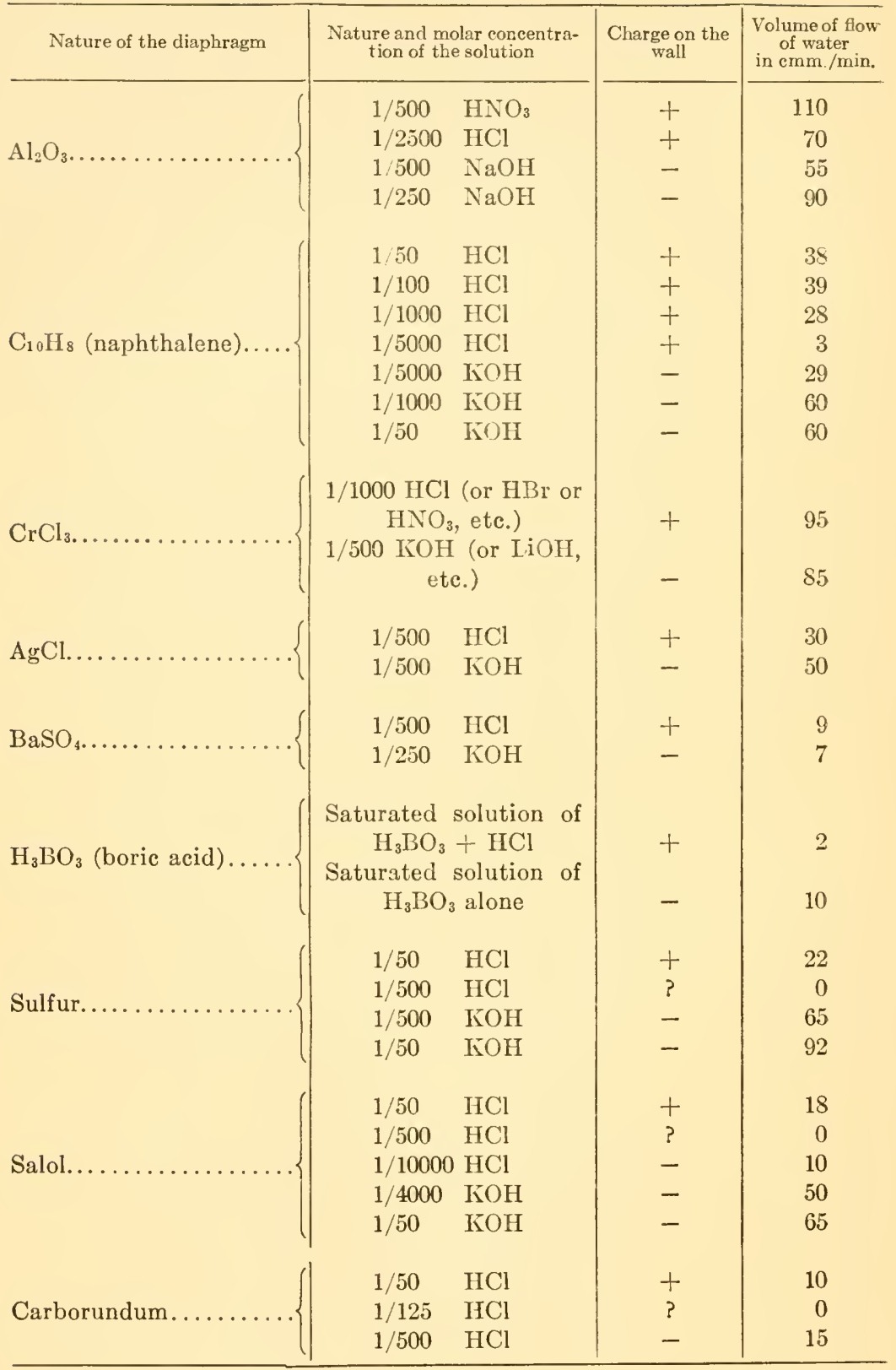


TABLE 38-Continued

\begin{tabular}{|c|c|c|c|}
\hline Nature of the diaphragm & $\begin{array}{l}\text { Nature and molar concentra- } \\
\text { tion of the solution }\end{array}$ & $\begin{array}{l}\text { Charge on the } \\
\text { wall }\end{array}$ & $\begin{array}{l}\text { Volume of flow } \\
\text { of water } \\
\text { in cc./min. }\end{array}$ \\
\hline Carborundum....... & $\begin{array}{ll}\text { Distilled water } \\
1 / 5000 & \mathrm{KOH} \\
1: 500 & \mathrm{KOH}\end{array}$ & $\begin{array}{l}- \\
- \\
-\end{array}$ & $\begin{array}{r}50 \\
60 \\
105\end{array}$ \\
\hline Gelatin... & $\begin{array}{ll}1 / 50 & \mathrm{HCl} \\
1 / 100 & \mathrm{KOH}\end{array}$ & $\begin{array}{l}+ \\
-\end{array}$ & $\begin{array}{l}22 \\
35\end{array}$ \\
\hline Cellulose... . & $\begin{array}{ll}1 / 50 & \mathrm{HCl} \\
1 / 500 & \mathrm{HCl} \\
1 / 500 & \mathrm{KOH}\end{array}$ & $\begin{array}{l}? \\
- \\
-\end{array}$ & $\begin{array}{r}0 \\
20 \\
70\end{array}$ \\
\hline
\end{tabular}

It is to be noted that he had observed that the reversal of the electric charge did not always occur exactly at the neutral reaction. This is most clearly shown in the table in the case of cellulose, on which, as well as on iodoform, no positive charge could be produced even in a $1 / 30 \mathrm{~N} \mathrm{HCl}$ solution. An exact interpretation of these phenomena is evidently afforded by the same considerations as those applied to the theory of the isoelectric point for ampholytes in solution, which, at the time of Perrin's investigations, had not yet been developed. And yet Perrin was farsighted enough to regard the above given law as an approximation only, and as a strictly valid law he proposed the following:

The electric potential of any wall (diaphragm) toward a solution is always increased by the addition of an (univalent) acid and is always decreased by the addition of a (univalent) base.

\section{The isoelectric point of diaphragms; diaphragms with and without an isoelectric point}

Further investigations by Bethe and Toropoff, ${ }^{28}$ Glixelli29 and Gyemant, ${ }^{30}$ who employed somewhat different methods, permitting of the maintenance of an exact $\mathrm{pH}$ at the electrodes, showed that it was in principle quite incorrect to expect the reversal of the charge

${ }^{28} \mathrm{~A}$. Bethe and Th. Toropoff, Zeitschr. f. physikal. Chem. 88, $6 S 6$ (1914) and 89,597 (1915). 102.

${ }^{29}$ S. Glixelli, Bull. de l'Acad. des Sciences de Cracovie. Série A. 1917, p.

${ }^{30}$ A. Gyemant, Kolloid-Zeitschr. 28, 103 (1921). 
to occur in general at the neutral reaction. To be sure, for a few substances, such as charcoal and gelatin, the reversal at neutrality was to a certain extent confirmed. But a large series of substances was found which at ever so strong an acid reaction showed no positive charge, but which at most showed an abolition of their original negative charge, as for instance, cellulose, collodion, agar, kaolin, also retort-charcoal belongs to this group, according to Bethe and Toropoff. Even the point of reversal of the charge of gelatin (i.e.,

TABLE 39

\begin{tabular}{|c|c|c|c|}
\hline $\mathrm{BeO}$ & $\begin{array}{c}V \\
\text { Velocity of } \\
\text { endosmosis }\end{array}$ & Silicic acid $\left(\mathrm{SiO}_{2}, \mathrm{nH}_{2} \mathrm{O}\right)$ & $\begin{array}{c}V \\
\text { Velocity oi } \\
\text { endosmosis }\end{array}$ \\
\hline Distilled water.. & -1.59 & Distilled water. & +0.63 \\
\hline $0.0001 \mathrm{~N} \mathrm{NaOH..}$ & -0.87 & $0.03 \mathrm{NHNO}_{3}$. . & +0.33 \\
\hline $0.00016 \mathrm{~N} \mathrm{NaOH}$. & +0.21 & $0.05 \mathrm{~N} \mathrm{HCl} .$. & +0.30 \\
\hline $0.001 \mathrm{~N} \mathrm{NaOH} . .$. & +1.84 & $0.47 \mathrm{~N} \mathrm{HNO}_{3} \ldots$ & +0.12 \\
\hline $\mathrm{ZnO}$ & & $\mathrm{Sb}_{2} \mathrm{O}_{5}$ & \\
\hline Distilled water... & -0.31 & Distilled water.. & +1.27 \\
\hline $0.0002 \mathrm{~N} \mathrm{NaOH}$. & -0.22 & $0.076 \mathrm{~N} \mathrm{HCl.} \mathrm{.}$ & +1.27 \\
\hline $0.0003 \mathrm{~N}$ NaOH.. & +0.16 & $0.63 N \mathrm{HCl} \ldots . . .$. & +1.08 \\
\hline $0.0004 N \mathrm{NaOH} . .$. & +0.24 & & \\
\hline $\mathrm{Mg}(\mathrm{OH})_{2}$ & & Tungstic acid $\left(\mathrm{H}_{2} \mathrm{WO}_{4}\right)$ & \\
\hline $0.0006 \mathrm{~N} \mathrm{NaOH}$. & -0.64 & Distilled water.. & +2.66 \\
\hline $0.01 \mathrm{~N} \mathrm{NaOH} . .$. & -0.05 & $0.045 N \mathrm{HCl} . .$. & +1.12 \\
\hline $0.02 N \mathrm{NaOH} .$. & +0.90 & $0.207 N \mathrm{HCl} . .$. & +0.28 \\
\hline & & $0.8 N \mathrm{HCl} . .$. & 0 \\
\hline \multicolumn{2}{|c|}{$\begin{array}{l}\text { The isoelectric point is at a definite } \\
\mathrm{pH}-\text { (mostly at an alkaline reac- } \\
\text { tion). }\end{array}$} & \multicolumn{2}{|c|}{$\begin{array}{l}\text { No reversal of charge even in an } \\
\text { extremely acid reaction. }\end{array}$} \\
\hline
\end{tabular}

gelatinized collodion membranes as used by Jacques Loeb ${ }^{31}$ or membranes of congealed gelatin) proved to be not at exact neutrality but rather at that $\mathrm{pH}$ which the present author ${ }^{32}$ had previously found by electrophoresis experiments to be the isoelectric point of gelatin.

Table 39 shows some of the results obtained by Glixelli. ${ }^{29} \mathrm{He}$ found at first a few substances which, as most of Perrin's substances did, showed a reversal of their charge on passing from an acid to an

31 Jacques Loeb, Journ. of gen. physiol. 2, 225 (1920).

${ }^{32}$ L. Michaelis and W. Grineff, Bioch. Zeitschr. 41, 373 (1912). 
alkaline reaction, but it is quite clear that this reversal does not in general occur at exact neutrality.

The investigations by Gyemant ${ }^{30}$ in the author's laboratory yielded results given in table 40 (pp. 262-3).

77. Application of the acidoid theory to the sign of charge on diaphragms

The profound influence of the $\mathrm{H}$ - and $\mathrm{OH}-\mathrm{ions}$ which Perrin found in his investigations, led him to the assumption that the electric double layer is always formed only by the ions of the water, one layer consisting of $\mathrm{H}$-ions and the other of $\mathrm{OH}$-ions. He believed that other (univalent) ions do not participate in the formation of the double layer. Let us accept Perrin's assumption for the time being, just as in the development of the theory of dissolved electrolytes we started out with the simplifying assumption that no cations competed with $\mathrm{H}$-ions and no anions competed with the $\mathrm{OH}$-ions. Now, by combining Perrin's assumption with the theory of ionic adsorption, we may arrive at the following conception: The charge upon any solid wall depends upon the fact that it adsorbs $\mathrm{H}$ - and $\mathrm{OH}$-ions to different extents. A wall which adsorbs only $\mathrm{OH}$-ions can therefore acquire only a negative charge (cellulose, collodion, agar); a wall which is capable of adsorbing only $\mathrm{H}$-ions can have only a positive charge (such are hitherto unknown). A wall which can adsorb either of the two jons will be either positive or negative, depending on the reaction of the adjacent solution. The point of reversal of the charge need not lie at the neutral reaction. This can only happen when the wall has, at the neutral reaction, an equal capacity to adsorb $\mathrm{H}$ - and $\mathrm{OH}$-ions. We have shown this condition to obtain quite closely in the case of charcoal. In the case of gelatin the relationship is as follows. Its isoelectric point is at $\left[\mathrm{H}^{+}\right]=2.10^{-5}$. At greater values of $\left[\mathrm{H}^{+}\right]$it adsorbs H-ions. Hence it can be said that the gelatin wall consists of electroneutral molecules which acquire an electric charge by adsorbing $\mathrm{H}$-ions; or, that the wall consists of positive gelatin ions, for a molecule which had bound an $\mathrm{H}$-ion in acquiring its charge is according to our earlier definition (page 16) a positive ion. When the $\left[\mathrm{H}^{+}\right]$is less than that corresponding to the isoelectric point, hence the $\left[\mathrm{OH}^{-}\right]$is greater, the gelatin binds $\mathrm{OH}$-ions and acquires a negative charge; which is the equivalent of saying that the gelatin 


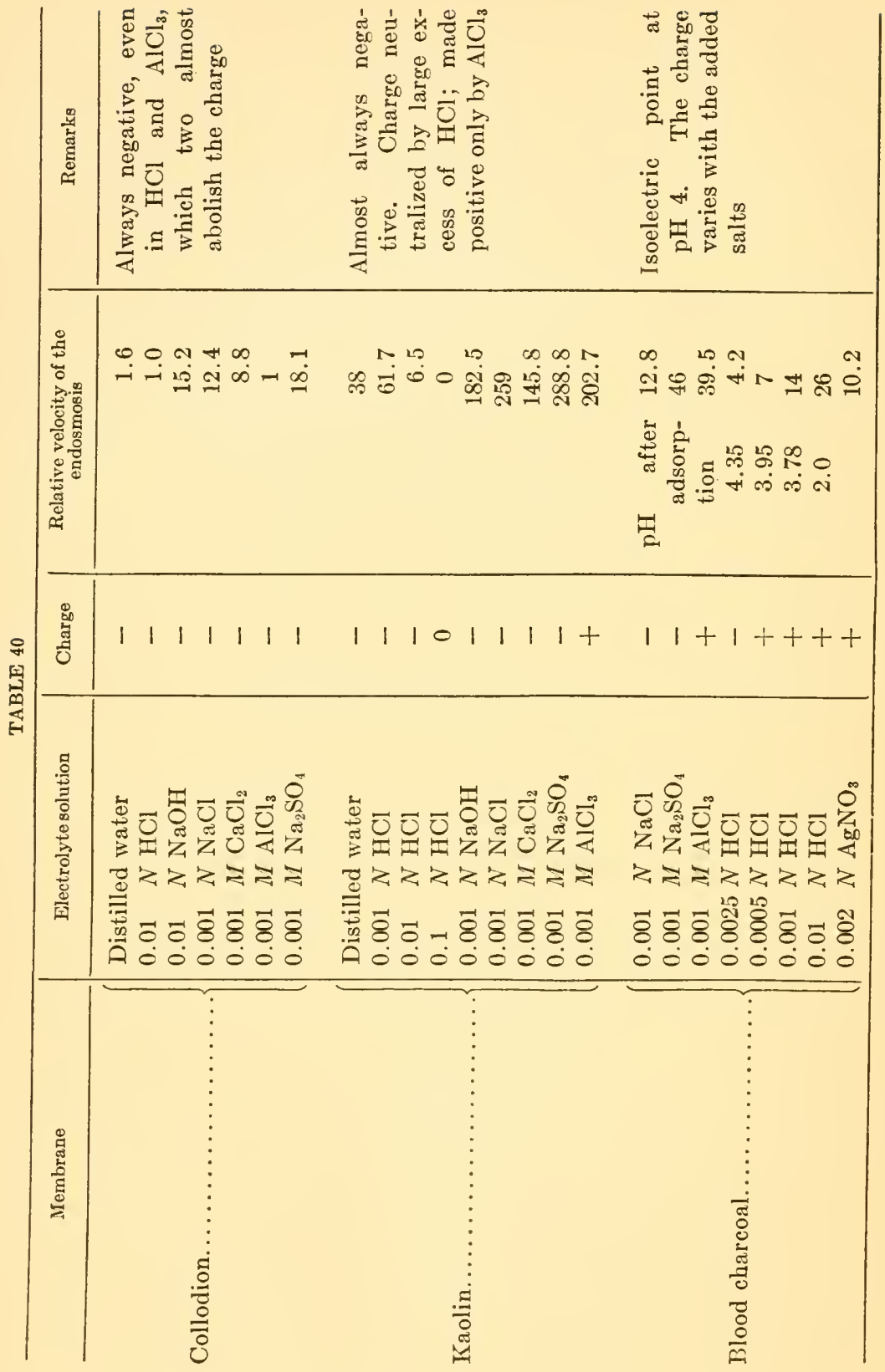


ADSORPTION POTENTIALS AND ELECTROKINETIC PHENOMENA 263

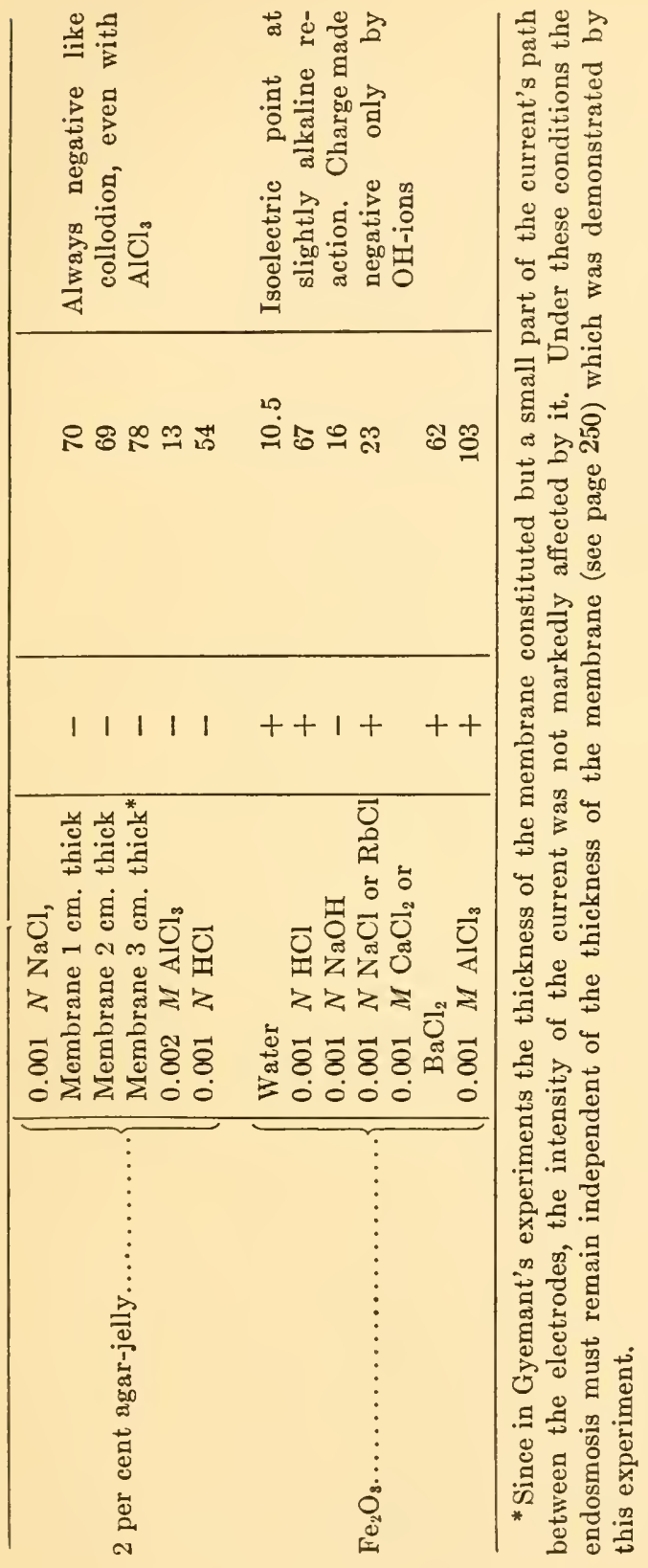


dissociates off $\mathrm{H}$-ions, which, in turn, go to form the external stratum of the double layer, while the negatively charged ions form the inner layer.

A gelatin ion which had combined with an $\mathrm{H}$-ion (or, starting from the electroneutral hydrated form, which had split off an $\mathrm{OH}$-ion) can no longer be designated as "a gelatin membrane with a covering layer of H-ions," just as an H-ion bound to an electroneutral molecule $\left(\mathrm{H}^{+}+\mathrm{NH}_{3}=\mathrm{NH}_{4}{ }^{+}\right)$is no longer designated as an $\mathrm{H}$-ion. Thus it may appear somewhat forced, in adhering to Perrin's conception, to state that the charge on a wall or membrane depends only upon a covering layer of $\mathrm{H}$ - or $\mathrm{OH}$-ions. But in reality there is no contradiction in this; it is merely a purely formal difference of expression. If we should decide to accept the $\mathrm{NH}_{4}{ }^{+}$-ion as an $\mathrm{NH}_{3}$ molecule overlaid by an $\mathrm{H}^{+}$-ion, then Perrin's interpretation becomes also acceptable for gelatin or any other charged wall. But this mode of expression is barely suitable. In order to state the basic idea underlying Perrin's rule in a form adequate for our present day theory, we may say that: A wall becomes electrically charged, either by binding (adsorbing) $\mathrm{H}$-ions while acquiring the charge; this is equivalent to saying that it dissociates off $\mathrm{OH}$-ions, which then form an ionic layer at a finite distance from the wall; or by binding (adsorbing) $\mathrm{OH}$-ions; which is equivalent to stating that it dissociates off $\mathrm{H}$-ions which aggregate into a layer at a finite distance from the wall.

By combining this conception with the definitions given above in section 67 , this law assumes the following form:

A solid wall is always positively charged with respect to an aqueous solution, if the substance of the wall is an acidoid. It is always negatively charged, if it is a basoid. A wall may be either positively or negatively charged, depending upon the hydrion concentration of the solution, if its substance is an ampholytoid. Just as in the case of a true ampholyte, zero charge on an ampholytoid is present oniy at its isoelectric point, the charge varying directly with the hydrion concentration. The isoelectric point of the wall, depending upon the chemical nature of its substance, may lie at an acid, neutral or alkaline reaction, and it is represented by the middle point of a more or less wide isoelectric zone.

In the case of the dissolved ampholyte, the width of this zone depends upon the value of the product $k_{\mathrm{a}} \times k_{\mathrm{b}}$. For the present no corresponding value for an ampholytoid can be defined. 
In general, the acidoid theory still shows a total lack of any values which would determine or define the relative strength of the acidoid or basoid properties in the sense in which the dissociation constant defines the strength of the various true acids and bases. In this latter case such a scale of measurement was made available by the possibility of applying the mass-law. This possibility is lacking in the case of acidoids and basoids. And yet it appears to be a quite soluble problem for the future, to find the means of extending the definition of the acidic and basic character of substances so as to include cases bordering upon the state of molecular dispersion.

All that is valid for solid walls is entirely applicable to any particles suspended in water and to colloidal solutions. And thus the electric charge phenomena of colloidal solutions are rendered completely analogous to the dissociation phenomena of electrolytes in true solution. The laws of dissociation hence appear as a limiting case for the condition where the dissolved electrolyte approaches molecular dispersion. On the other hand, the electric charge phenomena in a colloidal solution appear as a limiting case for an electrolyte solution in which the dissolved electrolyte become more coarsely dispersed and finally acquires the character of a solid "wall."

\section{A consideration of the other ions}

The above simple approximate method of explanation was made possible, as in the case of theory of electrolytic dissociation, by the auxiliary assumption which ascribed an exceptional position to the $\mathrm{H}$ - and $\mathrm{OH}$-ions. Just as in that case we assumed that the salts of acids and bases are always completely dissociated, or, in effect, nonexistent, so in this case we assume provisionally that a wall always adsorbs only $\mathrm{H}$ - and $\mathrm{OH}-$ ions, but no $\mathrm{Na}$ - or Cl-ions, for instance. The extremely slight adsorbability of $\mathrm{NaCl}$ on charcoal (see page 234) in contrast to $\mathrm{NaOH}$ or $\mathrm{HCl}$, indicates that such an assumption must be justifiable in reaching an approximated general law. In order to complete the scope of this law it is again necessary to drop these simplifying assumptions and to reconsider it in the light of the adsorability of the other ionic species. It is to be expected that the influence of any ionic species is the more pronounced, the greater its adsorbability, and hence the more effectively it can compete with the H- or OH-ions. Accordingly, the common univalent ions, which are poorly adsorbed, have also the least influence upon the electric 
charge on a solid wall. And yet in higher concentrations of univalent ions this effect is markedly present. This is shown in the following experiment by Perrin. The figures give the electroendosmosis velocity together with the sign of the charge.

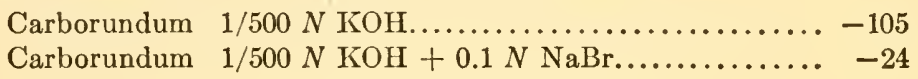

$\mathrm{CrCl}_{3} \quad 1 / 150 \mathrm{~N} \mathrm{HCl} \ldots \ldots \ldots \ldots \ldots \ldots \ldots \ldots \ldots \ldots \ldots \ldots+100$

$\mathrm{CrCl}_{3} 1 / 150 N \mathrm{HCl}+0.1 \mathrm{~N} \mathrm{KBr} \ldots \ldots \ldots \ldots \ldots \ldots \ldots \ldots+35$

In describing adsorption it was stated (page 237) that the adsorption of $\mathrm{HCl}$ or of $\mathrm{NaOH}$ is augmented by the addition of a neutral salt. This was explained in the sense that the forces opposing the isolated adsorption of the $\mathrm{H}$ - (or OH-) ions were thereby diminished. Another explanation of the same phenomenon is suggested, as can be seen from these experiments, in the diminution by the presence of the neutral salt of the potential which develops in the adsorption of $\mathrm{HCl}$ or $\mathrm{KOH}$. An analytic formulization of the regularity of this phenomenon has not yet been attempted.

According to the above the decrease of potential by univalent ions could be explained without regard for their adsorbability. But they doubtlessly do exhibit an adsorbability, be it ever so slight, and they do enter into competition to a small extent with the $\mathrm{H}$ - and $\mathrm{OH}$-ions for adsorption on boundary surfaces.

This competing effect is more striking in the case of the much more strongly adsorbable polyvalent ions, as shown again by the following of Perrin's figures:

(a) $\mathrm{Al}_{2} \mathrm{O}_{3} \quad 1 / 1000 \mathrm{~N} \mathrm{HNO}_{3} \ldots \ldots \ldots \ldots \ldots \ldots \ldots \ldots \ldots \ldots \ldots+100$

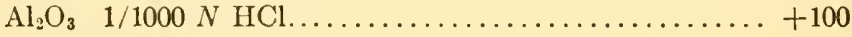

$\mathrm{Al}_{2} \mathrm{O}_{3} 1 / 1000 \quad N \mathrm{H}_{2} \mathrm{SO}_{4} \ldots \ldots \ldots \ldots \ldots \ldots \ldots \ldots \ldots \ldots+15$

(b) $\mathrm{CrCl}_{3} \quad 1 / 1000 N \mathrm{HNO}_{3} \ldots \ldots \ldots \ldots \ldots \ldots \ldots \ldots \ldots \ldots+88$

$\mathrm{CrCl}_{3} \quad 1 / 1000 \mathrm{~N} \mathrm{HNO}_{3}+1 / 1000 N \mathrm{MgSO}_{4} \ldots \ldots \ldots \ldots \ldots+23$

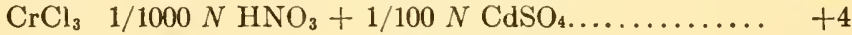

(c) $\mathrm{CrCl}_{3}$ Very dilute $\mathrm{HCl} . \ldots \ldots \ldots \ldots \ldots \ldots \ldots \ldots \ldots \ldots \ldots \ldots \ldots \ldots+75$

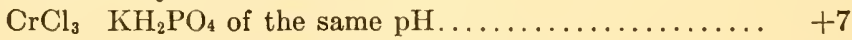

(d) $\mathrm{CrCl}_{3}$ in faintly alkaline water................. -46

$\mathrm{CrCl}_{3}$ Same $+1 / 1000 M \mathrm{~K}_{3} \mathrm{Fe}(\mathrm{CN})_{6} \ldots \ldots \ldots \ldots \ldots \ldots . .46$ 
(e) $\mathrm{CrCl}_{3}$ in faintly acidified water................. +59

$\mathrm{CrCl}_{3}$ Same $+1 / 1000 M \mathrm{~K}_{3} \mathrm{Fe}(\mathrm{CN})_{6} \ldots \ldots \ldots \ldots \ldots \ldots+2$

$\mathrm{CrCl}_{3}$ Same $+1 / 50 \quad M \mathrm{~K}_{3} \mathrm{Fe}(\mathrm{CN})_{6} \ldots \ldots \ldots \ldots \ldots \ldots-20$

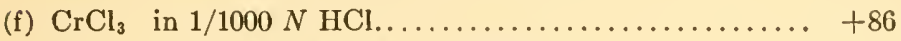

$\mathrm{CrCl}_{3}$ Same $+1 / 2000 M \mathrm{~K}_{4} \mathrm{Fe}(\mathrm{CN})_{6} \ldots \ldots \ldots \ldots \ldots \ldots+1.5$

These experiments show the effect of various polyvalent ions. As seen in (d), as long as a wall remains negatively charged, even polyvalent negative ions are without effect. But the same ions exercise, even in low concentrations, a strongly diminishing effect upon the potential of a positively charged wall, as in (e) and (f); while in greater concentration they can even reverse the sign of the charge, as in (e). This activity rises markedly with the valence. Bivalent ions are more effective than univalent, and trivalent ions have a greater effect than the bivalent. The effect of the valence of the anions is also noticeable in experiment (a), where the anion is added not in the form of a neutral salt, but as a constituent of the acid itself, for $\mathrm{HCl}$ and $\mathrm{HNO}_{3}$ show the same activity, while $\mathrm{H}_{2} \mathrm{SO}_{4}$ greatly reduces the potential.

The cations behave in an exactly corresponding manner: they exert an influence only upon a negatively charged wall. For example:

$\mathrm{CrCl}_{3}$ in faintly acidified water..................... +43

$\mathrm{CrCl}_{3}$ Same $+1 / 1000 M \mathrm{MgCl}_{2} \ldots \ldots \ldots \ldots \ldots \ldots \ldots \ldots+43$

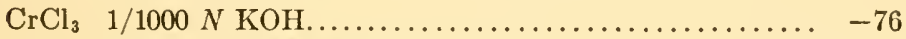

$\mathrm{CrCl}_{3}$ Same $+1 / 1000 M \mathrm{MgCl}_{2} \ldots \ldots \ldots \ldots \ldots \ldots \ldots \ldots,-10$

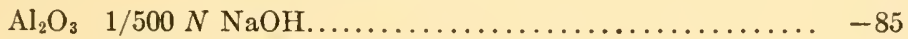

$\mathrm{Al}_{2} \mathrm{O}_{3}$ Same $+1 / 500 \mathrm{~N} \mathrm{Ca}\left(\mathrm{NO}_{3}\right)_{2} \ldots \ldots \ldots \ldots \ldots \ldots \ldots \ldots \ldots$

$\mathrm{Al}_{2} \mathrm{O}_{3}$ in faintly acidified water.................... +41

$\mathrm{Al}_{2} \mathrm{O}_{3}$ Same $+1 / 500 \mathrm{~N} \mathrm{Ca}\left(\mathrm{NO}_{3}\right)_{2} \ldots \ldots \ldots \ldots \ldots \ldots \ldots \ldots+41.5$

$\mathrm{Mn}_{2} \mathrm{O}_{3}$ in faintly alkaline water.................... -40

$\mathrm{Mn}_{2} \mathrm{O}_{3}$ Same $+1 / 500 \mathrm{~N} \mathrm{Ba}\left(\mathrm{NO}_{3}\right)_{2} \ldots \ldots \ldots \ldots \ldots \ldots \ldots \ldots+18$

Carborundum $1 / 500 \mathrm{~N}$ KOH...................... -60

Carborundum Same $+1 / 1000 \mathrm{~N} \mathrm{La}\left(\mathrm{NO}_{3}\right)_{3} \ldots \ldots \ldots \ldots \ldots \ldots, \quad-0.7$

The example with the $\mathrm{Mn}_{2} \mathrm{O}_{3}$ shows that the bivalent $\mathrm{Ba}$-ion can even reverse the charge. The same could be demonstrated to an even greater extent by $\mathrm{Al}^{+++}$or $\mathrm{La}^{+++}$, but these are so insoluble in 
alkaline solutions that it is impossible to obtain the concentrations of these ions necessary for the reversal of the charge. We shall later discuss in greater detail the profound influence of the trivalent ions upon the sign of the charge, under other experimental conditions.

It follows from the investigations of other authors that those diaphragms which do not become positive even in an extremely acid reaction fall into two groups: 1 . those which are positively charged by trivalent cations $\left(\mathrm{Al}^{+++}, \mathrm{La}^{+++}\right)$, and 2 . those the sign of whose charge cannot even be reversed by such trivalent cations. From the table given on pages $262-3$, taken from Gyemant's study, it is seen that while agar and collodion are not positively charged by $\mathrm{Al}^{+++}$, the charge of kaolin is reversed by this cation. In regard to collodion this is a confirmation of Jacques Loeb's findings. ${ }^{31}$ The reversal of the charge on kaolin appears to be easily explicable. Its surface adsorbs $\mathrm{Al}^{+++}$by exchange for an $\mathrm{H}$-atom of its silicic acid and forms, to a certain extent, a basic silicate, i.e., an H-atom of the silicic acid (of the kaolin) is replaced by an $\mathrm{Al}^{+++}$ion, which then still has two free valences left, or, because of its dissociated state, this amounts to two free positive charges. In the case of agar or collodion this is not possible, for these substances do not possess a replaceable $\mathrm{H}$-atom, and their negative charge can not be due to the splitting off of $\mathrm{H}$-ions, but rather to the adsorption of H-ions. (This is possibly a case of “apparent adsorption" cf. page 234). Heesch, ${ }^{33}$ working in Höber's laboratory, found similarly that agar membranes could not become positively charged, even by $\mathrm{La}^{+++}$, while he could demonstrate a reversal of the sign of the charge on parchment by $\mathrm{La}^{+++}$or by other strongly adsorbable organic cations (histone, clupein, Rhodamin S).

The otherwise irreversible sign of charge of such substances as collodion can still be reversed by $\mathrm{Al}^{+++}$when these diaphragms are covered by an adsorption layer of an amphoteric colloid. Thus J. Loeb $^{31}$ showed that collodion impregnated with a solution of gelatin or albumin can have the sign of its charge reversed by $\mathrm{H}^{+}$or $\mathrm{Al}^{+++}$, as would be the case for these two colloids taken alone.

Investigations on electrophoresis show it to be, as was to be expected, an exact reversal of electroendosmosis. The same material which, when used as a diaphragm, propels water towards the cathode, when it is suspended in water, migrates towards the anode. The phenomenon of cataphoresis leads so directly over into the theory of

${ }^{33}$ Heesch, Pflügers Arch. f. d. ges. Physiol. 190, 210 (1920). 
colloids that we shall discuss its contributions in greater detail in the following volume on colloids. For the present we shall only point out some instructive examples of the reversal of the sign of the charge. R. Höber ${ }^{34}$ established the fact that the ordinarily negatively charged blood corpuscles become easily positively charged under the influence of trivalent ions. But since their charge is also reversed by $\mathrm{H}$-ions, they are, therefore, of ampholytoid nature. ${ }^{35}$ Putter $^{30}$ found that bacteria in colloid-free aqueous suspension are always negatively charged, even in a most acid medium; and yet their charge is reversed by $\mathrm{Al}^{+++}$. But in the presence of peptone bacteria become also positively charged by means of high $\mathrm{H}^{+}$ concentrations.

\section{Summary of the theory of formation of the electric charge on walls or diaphragms}

The electric charging of a wall towards a solution depends upon the differences of adsorbability of the various ions. The charge of the adsorbed ion is imparted to the wall. The serial sequence of adsorbability of the ions is the same as can be found with charcoal by chemical analysis. Since the $\mathrm{H}-$ and $\mathrm{OH}-$ ions are particularly strongly adsorbable, the formation of the charge can be approximately explained, in the absence of polyvalent or organic ions, in terms of the difference between the adsorbabilities of the $\mathrm{H}$ - and $\mathrm{OH}$-ions alone. On closer consideration the other ions must also be taken into account. The serial sequence of the anions is probably the same for all adsorbents, as for example (arranged in order of increasing adsorbability):

$$
\mathrm{Cl}^{-} \mathrm{I}^{-} \mathrm{SCN}^{-} \mathrm{OH}^{-}
$$

and similarly it is also the same for the cations, namely in the order:

$$
\mathrm{Na}^{+} \mathrm{Ca}^{++} \mathrm{Al}^{+++} \mathrm{H}^{+}
$$

But the absolute extent to which anions on the one hand and cations on the other are adsorbed by any adsorbent is quite different

${ }^{34}$ R. Höber, Pflügers Arch. f. d. ges. Physiol. 101, 627 (1904); 102, 196 (1904).

${ }^{35}$ L. Michaelis and Takahashi, Bioch. Zeitschr. 29, 439 (1910); Calvin B. Coulter, Journ. of Gen. Physiol. 3, 309 (1921).

${ }^{36}$ E. Putter, Zeitschr. f. Immunitiitsforsch. u. exp. Therap., 32, 538 (1921). 
for the different substances. Thus charcoal adsorbs $\mathrm{H}$ - and $\mathrm{OH}$-ion approximately equally well, and if we write down the two ionic series in such a way that equally adsorbable ions are placed one directly under the other, we obtain the following schematic serial arrangement (for charcoal)

$$
\begin{array}{cccc}
\mathrm{Cl} & \mathrm{I} & \mathrm{SCN} & \mathrm{OH} \\
\mathrm{Na} & \mathrm{Ca} & \mathrm{Al} & \mathrm{H}
\end{array}
$$

For $\mathrm{Al}_{2} \mathrm{O}_{3}$, which adsorbs $\mathrm{H}$-ions ${ }^{37}$ more strongly than $\mathrm{OH}-\mathrm{Hons}^{88}$ the arrangement will probably be

$$
\begin{array}{cccc}
\mathrm{Cl} & \mathrm{I} & \mathrm{SCN} & \mathrm{OH} \\
\mathrm{Na} & \mathrm{Ca} & \mathrm{Al} & \mathrm{H}
\end{array}
$$

For gelatin which adsorbs $\mathrm{OH}-$ ions $^{39}$ more strongly than H-ions: ${ }^{40}$

$$
\begin{array}{ccccc} 
& \mathrm{Cl} & \mathrm{I} & \mathrm{SCN} & \mathrm{OH} \\
\mathrm{Na} & \mathrm{Ca} & \mathrm{Al} & \mathrm{H} &
\end{array}
$$

For an ampholytoid adsorbent with even stronger acidic properties:

$$
\begin{array}{llllll} 
& & \mathrm{Cl} & \mathrm{I} & \mathrm{SCN} & \mathrm{OH} \\
\mathrm{Na} & \mathrm{Ca} & \mathrm{Al} & \mathrm{H} & &
\end{array}
$$

For an adsorbent which remains negatively charged even in an extremely acid reaction (collodion):

\section{$\mathrm{Na} \quad \mathrm{Ca} \quad \mathrm{Al} \quad \mathrm{H}$}

\section{$\mathrm{Cl}$ I $\mathrm{SCN} \quad \mathrm{OH}$}

i.e., the cations are displaced so far to the left in the series that their adsorbability may well be regarded as being equal to zero. There is no fundamental difference between a solid wall, the disperse particle of a colloidal solution and a single molecule in true solution. The process of adsorption for all these cases is a completely equivalent one, after it had become established that an $\mathrm{NH}_{3}$-molecule, a glycocoll molecule, a single casein molecule, a molecule superficially located on a colloidal casein aggregate, a molecule on the surface of charcoal, each of these merely binds an $\mathrm{H}$-ion.

$$
\begin{aligned}
& { }^{87} \mathrm{Al}_{2} \mathrm{O}_{3}+6 \mathrm{H}^{+}=2 \mathrm{Al}^{+++}+3 \mathrm{H}_{2} \mathrm{O} . \\
& { }^{38} \mathrm{Al}_{2} \mathrm{O}_{3}+6 \mathrm{OH}^{-}=2\left(\mathrm{AlO}_{3}\right)=+3 \mathrm{H}_{2} \mathrm{O} . \\
& { }^{39} \mathrm{HRNH}_{2}+\mathrm{H}^{+}=\left(\mathrm{HRNH}_{3}\right)^{+} . \\
& { }^{\circ} \mathrm{HRNH}_{2}+\mathrm{OH}^{-}=\left(\mathrm{RNH}_{2}\right)^{-}+\mathrm{H}_{2} \mathrm{O} .
\end{aligned}
$$


The absolute amount of adsorbed $\mathrm{H}$ - or $\mathrm{OH}$-ions depends upon their concentration. That hydrion concentration at which $\mathrm{H}$ - and $\mathrm{OH}$-ions are equally well adsorbed, in the absence of other strongly adsorbable ions, is designated as the isoelectric point of the adsorbent. If it is at some finite $\left[\mathrm{H}^{+}\right]$then we are dealing with an ampholyte or ampholytoid. If it is at $\left[\mathrm{H}^{+}\right]=\infty$, then the substance is an acid or an acidoid, and, if it is at $\left[\mathrm{OH}^{-}\right]=\infty$, the substance is a base or a basoid.

As soon as, in addition to the $\mathrm{H}$ - and $\mathrm{OH}-$ ions, still other ions participate in the adsorption (i.e., poorly adsorbable ions in high concentrations, or strongly adsorbable ions in any concentration) the relations become more or less different. The presence of well adsorbable cations had the same effect as an increase in the $\left[\mathrm{H}^{+}\right]$, while the presence of well adsorbable anions, analogously, as an increase in the $\left[\mathrm{OH}^{+}\right]$.

The reduction of these laws to the limiting case, when $\mathrm{H}$ - and $\mathrm{OH}-i o n s$ only are present as actively participating ions, is surely but an arbitrary or optional method. Theoretically one could start with any other pair of ions. But this representation has the advantage that the ideal limiting case-absence of other active ions-can be readily realized, which would not be possible for any other single pair of ions. This arbitrariness is no greater than that involved in ascribing to the acids and bases alone of all the electrolytes an exceptional position.

\section{Changes in concentration, especially of $\mathrm{H}$-ions, in electro- endosmosis}

In comparing Helmholtz's theory of endosmosis with the modern conceptions developed later, certain incongruities become apparent. Helmholtz calculated from the potential difference between the porous diaphragm and the water, which he assumed as given, the amount of water transported by a given external electrical force. His internal friction factor enters into his equation only from the intrinsic properties of the water. Hence it appears as if any electrolytes dissolved in the water, which scarcely affect its viscosity, can influence the process of endosmosis only insofar as they may alter the potential of the diaphragm. But if we accept our above developed view that the current of the external forces acts primarily upon the movable ionic layer, and that the latter only carries the water along, 
then we must consider that the amount of water carried along by an ion depends upon the so-called hydration of the ionic species involved (see page 123). Hence we must also consider that the amount of water transported in endosmosis at a given wall potential depends at least as much upon the water binding capacity of the ionic species passing along the wall as upon the internal friction of the water. Furthermore, Helmholtz's theory regards the water as a whole, and it does not admit the possibility that the various constituents of the solution, the water and the individual ionic species in solution, may be differently displaced in the process of endosmosis. The superiority of the newer conception could be proven if we succeded in demonstrating local changes in concentration of the dissolved ions through endosmosis. Although these changes in concentration had been previously described, ${ }^{41}$ the more exact and consistent study of this aspect of the problem was furnished in the important contributions by Bethe. ${ }^{42}$ The fundamental observation made by Bethe was the following. During the process of electroendosmosis through a collodion, parchment or gelatin membrane, or through a diaphragm of powdered material, the originally neutral solution on the anode side of the diaphragm became acidified while that at the cathode side it became alkaline (or the reverse of what happens in the course of ordinary electrolysis of a neutral solution between two platinum electrodes). A change in the concentrations of the other ions was also observed, but by far the greatest and the most easily demonstrable was the change in the concentrations of the $\mathrm{H}$ - and $\mathrm{OH}$-ions. This is but a natural consequence of the present day theory of endosmosis. Bethe and Toropoff developed this theory in the following way:

When, for example, the diaphragm wall is negatively charged, the anions are held in the pores in an adhering layer, and to these is due the negative charge on the wall. Above all others are the $\mathrm{OH}$-ions so held, then the polyvalent or the strongly adsorbable organic anions, while the common univalent anions are bound but to a very slight degree. Now, if the capillary spaces be only sufficiently small, then, under certain conditions, almost all $\mathrm{OH}$-ions, and possibly almost all

${ }^{41}$ Hittorf, Zeitschr. f. physikal. Chem. 39, 9, 613 (1901).

${ }^{42}$ A. Bethe, Zentralbl. f. Physiol. 23, 1909, No. 9; Internat. Physiol. Kongr. Wien, 1910; Munch. med. Wochnschr. 1911, No. 23; A. Bethe and Th. Toropoff, Zeitschr. f. physikal. Chem. 88, 686 (1914) and 89, 597 (1915). 
This yields as a balance B for the boundary layer:

$B=\left(m-m_{1}\right) K^{+}+\left(a_{1}-a\right) A^{-}+\left(n-n_{1}\right) H^{+}+\left(b_{1}-b\right) \mathrm{OH}^{-}$

or totalled in terms of molecules:

$$
\left.\begin{array}{rl}
\mathrm{B}=\left(\mathrm{m}-\mathrm{m}_{1}\right) \mathrm{KA}+\left(\mathrm{b}_{1}-\mathrm{b}\right) \mathrm{H}_{2} \mathrm{O} & +\left[\left(\mathrm{a}_{1}-\mathrm{a}\right)-\left(\mathrm{m}-\mathrm{m}_{1}\right)\right] \mathrm{A}^{-} \\
+ & {\left[\left(\mathrm{n}-\mathrm{n}_{1}\right)-\left(\mathrm{b}_{1}-\mathrm{b}\right)\right] \mathrm{H}^{+}}
\end{array}\right\}
$$

Since according to the original definition $m+n+a+b=m_{1}+n_{1}$ $+a_{1}+b_{1}$, consequently,

$$
\left(a_{1}-a\right)-\left(m-m_{1}\right)=\left(n-n_{1}\right)-\left(b_{1}-b\right)
$$

Hence

\begin{tabular}{|c|c|c|c|c|}
\hline \multicolumn{2}{|c|}{ Free solution } & \multirow{2}{*}{ 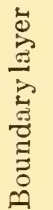 } & \multicolumn{2}{|c|}{ Diaphragm } \\
\hline pos. pole & $\begin{array}{l}+\mathrm{m} \mathrm{K}^{+} \rightarrow \\
+\mathrm{nH}^{+} \rightarrow \\
-\mathrm{a} \mathrm{A}^{-} \leftarrow \\
-\mathrm{b} \mathrm{OH}^{-} \leftarrow\end{array}$ & & $\begin{array}{l}\rightarrow \mathrm{m}_{1} \mathrm{~K}^{+}- \\
\rightarrow \mathrm{n}_{1} \mathrm{H}^{+}- \\
\leftarrow \mathrm{a} \mathrm{A}^{-}+ \\
\leftarrow \mathrm{b}_{1} \mathrm{OH}^{-}+\end{array}$ & neg. pole \\
\hline
\end{tabular}

$$
\mathrm{B}=\left(\mathrm{m}-\mathrm{m}_{1}\right) \mathrm{KA}+\left(\mathrm{b}_{1}-\mathrm{b}\right) \mathrm{H}_{2} \mathrm{O}+\left[\left(\mathrm{n}-\mathrm{n}_{1}\right)-\left(\mathrm{b}_{1}-\mathrm{b}\right)\right] \mathrm{HA} \text { (IV) }
$$

TABLE 41

HA is the free acid, IAA is the salt. Therefore, not only did a change in the concentration occur, but also free acid appears or disappears, according as to whether the sign of the factor before HA is positive or negative; this depends on the values of $n, n_{1}, b$ and $b_{1}$.

Equation II may also be stated in molecular terms as follows:

$$
\left.\begin{array}{rl}
\mathrm{B}=\left(\mathrm{a}_{1}-\mathrm{a}\right) \mathrm{KA}+\left(\mathrm{n}-\mathrm{n}_{1}\right) \mathrm{H}_{2} \mathrm{O}+ & {\left[\left(\mathrm{m}-\mathrm{m}_{1}\right)-\left(\mathrm{a}_{1}-\mathrm{a}\right)\right] \mathrm{K}} \\
+ & {\left[\left(\mathrm{b}_{1}-\mathrm{b}\right)-\left(\mathrm{n}-\mathrm{n}_{1}\right)\right] \mathrm{OH}}
\end{array}\right\}
$$

It follows from (1)

$$
\left(m-m_{1}\right)-\left(a_{1}-a\right)=\left(b_{1}-b\right)-\left(n-n_{1}\right)
$$

therefore

$$
\mathrm{B}=\left(\mathrm{a}_{1}-\mathrm{a}\right) \mathrm{KA}+\left(\mathrm{n}-\mathrm{n}_{1}\right) \mathrm{H}_{2} \mathrm{O}+\left[\left(\mathrm{b}_{1}-\mathrm{b}\right)-\left(\mathrm{n}-\mathrm{n}_{1}\right)\right] \mathrm{KOH}(\mathrm{VI})
$$

and from this we can obtain the increase or decrease in the amount of free base $\mathrm{KOH}$. 
In the case where all of the anions are fixed in the layer adhering to the wall, but where all of the cations are freely motile, we have

$$
\mathrm{m}_{1}+\mathrm{n}_{1}=1 ; \mathrm{a}_{1}=\mathrm{b}_{1}=0 ; \mathrm{m}_{1}>\mathrm{m} ; \mathrm{n}_{1}>\mathrm{n}
$$

and equation (VI) becomes

$$
\mathrm{B}=-\mathrm{aKA}-\left(\mathrm{n}_{1}-n\right) \mathrm{H}_{2} \mathrm{O}+\left(\mathrm{n}_{1}-\mathrm{n}-\mathrm{b}\right) \mathrm{KOH}
$$

If the solution is neutral to begin with then $\mathrm{n}$ and $\mathrm{b}$ must be small with respect to $\mathrm{n}_{1}$, because of the slight concentration of $\mathrm{H}$ - and $\mathrm{OH}-i o n s$, and the $\mathrm{KOH}$ term in (VI) will be positive, i.e., the solution will be alkaline on the anode side.

Similarly, under the same conditions, the diaphragm boundary on the cathode side must become acid. These predictions based on the theory correspond, for most of the neutral salts, with the experimental findings of Bethe and Toropoff.

The higher the concentration of the dissolved electrolytes, and, it may be added, the larger the capillary spaces, the less likely it is that the adsorbable ionic species will be completely bound on the walls of the pores; and, consequently, the smaller will be the changes in concentration produced by the passage of the current, and, as was pointed out above, the endosmotic transport of water resulting from concentration changes will be diminished.

If the sign of the charge on the diaphragm is irreversible, and the diaphragm is of an acidoid character (collodion), the walls of the pores adsorb only $\mathrm{OH}$-ions and other anions, but no cations. According to the adsorbability of the anions, other than $\mathrm{OH}^{-}$, which may be present, they are more or less bound by the wall and participate correspondingly more or less in the conductance of the current within the pores. The greater the adsorbability of the ions, other things being equal, the greater will be the change in hydrion concentration brought about on both sides of the diaphragm on the passage of the current. In fact, on comparing the effect of K- or Na-salts of the different anions in displacing the originally neutral reaction of a solution, Bethe and Toropoff found the following serial sequence, which was to be expected:

$$
\text { Citrate }{ }^{\equiv}>\mathrm{I}^{-}>\mathrm{Br}^{-}>\mathrm{SO}_{4}^{-}>\mathrm{Cl}^{-}>\mathrm{NO}_{3}^{-}
$$

When the anion was kept constant and the cations varied, no such sharply defined series could be obtained which would be independent 
of the anion used, at least not with collodion menbranes. Parchment membranes showed a distinct decreasing effect in the series:

$$
\mathrm{NH}_{4}>\mathrm{Li}>\mathrm{K}>\mathrm{Na}>\mathrm{Mg}>\mathrm{Ba}>\mathrm{Ca}
$$

This effect still needs further study.

Ampholytoid diaphragms showed, as they did also in respect to the endosmosis of water, a reversal of the effect when a certain well defined $\mathrm{H}^{+}$-concentration was exceeded. This $\left[\mathrm{H}^{+}\right]$represents in regard to changes in concentration a point of indifference. The experiments were carried out by Bethe and Toropoff especially on chromated gelatin, in which the disturbing swelling phenomena do not occur, as they do with ordinary gelatin. This point of indifference of concentration displacement was, as expected, different for different salts, and for $0.01 M$ solutions at the following $\mathrm{pH}$ values:

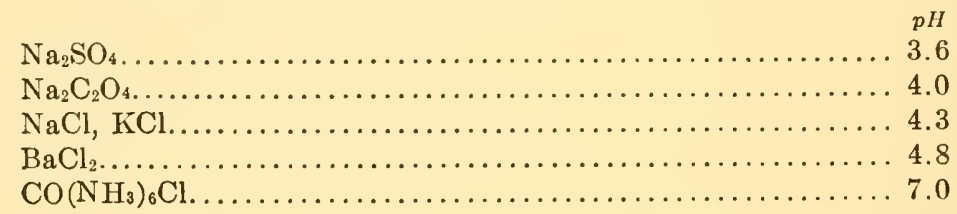

The important question arises as to the relation of this point of indifference to the isoelectric point. If we assume that the $\mathrm{pH}$ of the solution is equal to the isoelectric point of the diaphragm, then the wall of the diaphragm will not adsorb any ions, the potential of the wall is zero, and the current is conducted equally well within the pores as in the free solution. But when the water-combining power and also the mobility of the anions are equal to those of the cations in solution (or better, as in the case where the various cations are in the concentrations $k_{1}, k_{2}$. . . with the mobilities $u_{1}, u_{2}$... , and the anions are in the concentrations $a_{1}, a_{2}$... with the mobilities $v_{1}, v_{2}$. . . ; and when $u_{1} k_{1}+u_{2} k_{2}=v_{1} a_{1}+v_{2} a_{2}$. . .), no water will be transported. Otherwise, even at the isoelectric point, some, even if slight, displacement of water will occur. The point of indifference for the motility of the water is not quite equal to the isoelectric point, the latter corresponding more closely to the point of indifference for concentration change. In fact, Bethe and Toropoff demonstrated that the indifference points for water mobility and for concentration change do not always coincide. A total coincidence of these two points is possible only when the dissolved electrolyte com- 
bines these two properties: (1) neither of its ions must have a marked degree of adsorbability as compared to $\mathrm{H}$ - and $\mathrm{OH}$-ions, the mobilities of its two ions must be equal to each other. Of all the electrolytes $\mathrm{KCl}$ fulfills these conditions best, other binary univalent electrolytes, such as $\mathrm{NaCl}$, only to a certain extent, but $\mathrm{Na}_{2} \mathrm{SO}_{4}$, for example, not at all. All of the authors mentioned before considered the indifference point of water mobility as the isoelectric point of the diaphragm, but according to Bethe and Toropoff this is not quite correct.

\section{History of the theory of adsorption potentials}

The theory of adsorption potentials, as it is developed in this section, is not to be found in this form in the literature. The fact that ions may become adsorbed is quite obvious and was stated by a number of authors, without there being any evident historic sequence on this point. The present day theory dates back chiefly to two sources, and may be thought of as the result of the blending of the two, as it were. One is the earlier description by the present author ${ }^{43}$ and the other was that given by Freundlich. ${ }^{44}$ Beginning with the first, Michaelis, on the basis of a theoretical research of Nernst's, ${ }^{45}$ conceived a colloidal particle suspended in water, for example of a resin acid (mastic), as of a "binary electrode," which, in distinction to a simple electrode, tends to send into solution not one ion species only, but $\mathrm{H}$-ions and acid anions simultaneously. From the different solution tensions of these two kinds of ions taken in conjunction with the differing concentrations in which these two ions are present in a state of true solution in the aqueous phase, the author derived the potential of the double layer at the interface. Should there be present in the solution any species of ion which is capable of forming a salt of this acid, an ionic exchange occurs in the double layer which alters its potential. Already at that time the author explained this ionic exchange by stating that it is identical with ionic adsorption.

In a theoretical derivation of the ionic equilibrium the author ${ }^{43}$

${ }^{43}$ L. Michaelis, Physik. Chemie der Kolloide, in Richter-Koranyis Handbuch: Physikalische Chemie und Medizin. Leipzig 1908. L. Michaelis, Dynamik der Oberflächen. Dresden 1909. P. $49 \mathrm{ff}$.

${ }^{44}$ H. Freundlich, Kapillarchemie. Leipzig 1909. p. $213 \mathrm{ff}$.

${ }^{45}$ W. Nernst, Zeitschr. f. physikal. Chem. 9, 137 (1892).

${ }^{16}$ L. Michaelis, Zeitschr. f. Elektrochemie 14, 353 (1908). 
made the error of colrelating the solution tensions of the ions with their mobility, which error was pointed out by F. Haber. ${ }^{47}$ The solution tension bears no relation to the mobility of the ion.

Freundlich's conception is based on the idea that the ions, as well as the ordinary molecules, may be adsorbed on boundary surfaces, and that the adsorbabilities of the anion and of the cation of an electrolyte are unequal. He also ascribed a particularly high degree of adsorbability to the $\mathrm{H}$ - and $\mathrm{OH}$-ions. He derived the adsorption potential from the different adsorption tendencies of the anions and cations. To the adsorbents, according to their chemical (acidic or basic) character, he ascribed the tendency to dissociate off $\mathrm{H}$ - or $\mathrm{OH}$-ions respectively, which ions would then lead to the formation of the double layer. At this juncture Freundlich fell into the same error as Michaelis did, in correlating the splitting off of these ions with their high mobilities. But Freundlich showed at the same time that the adsorption potential need not be identical with the potential which the same adsorbent (when it is a metal, for example) would have towards the same solution, when it is used as a pole of a galvanic chain. This point must be recognized as being of very great importance, and this phenomenon will become more comprehensible in section 85 on "The Splitting of Potentials."

In general there does not appear to be any great difference between Freundlich's conception and that of the author. However, one difference between the two does appear in the following respect: Freundlich takes pain to maintain as far as possible a line of demarcation between chemical reaction and adsorption on boundary surfaces. He regards the adsorbent chiefly as a means of increasing the surface of the solution, and he does not attribute any particular value to the chemical nature of the adsorbent. This idea appears more distinctly in his chapters on the ordinary adsorption of non-electrolytes than it does in the above cited pages dealing with adsorption potentials. In this latter chapter Freundlich cannot help attributing a special importance to the acidic or basic nature of the adsorbent. And yet it does not appear from this chapter that he thereby relinquishes his sharp line of demarcation between adsorption and chemical reaction. The present author, on the contrary, took pains at the outset to remove the contrast between adsorption and chemical reaction, at least in the case of chemically reactive (acidic or basic) adsorbents.

${ }^{47}$ F. Haber, Ann. d. Physik. [4], 927 (1908); s. p. 948. 
The danger of delimiting chemical from physical attractions is obvious from the very nature of these processes, and a serviceable theory is quite evidently one which does not demand any such demarcation. It is under the influence of these ideas that this attempt is made to present a unified theory of adsorption potentials.

\section{Hydrodynamic potentials}

It has been previously stated (page 246) that in the study of adsorption potentials both methods of electroendosmosis and electrophoresis can be used. To these we must add two other methods, that of the hydrodynamic current and that of the current of falling particles.

When water is forced through a porous membrane or through a capillary tube, there arises a potential difference between the two sides of the membrane or between the two ends of the capillary tubes. This phenomenon is the reverse of electroendosmosis. For in the latter water is forced through a diaphragm by the application of a potential difference between its two ends, while in the former the potential difference is produced on forcing water through the diaphragm. If the two ends of the capillary are led off by means of metal electrodes, a current is obtained as long as the flow of water through the capillary continues. Hence such electric currents are designated as hydrodynamic currents, and the potentials which cause it are called hydrodynamic potentials. ${ }^{48}$ This phenomenon was discovered by Quincke. ${ }^{49}$ A porous clay plate was cemented between the bevelled ends of two glass tubes, or a diaphragm of some powdered substance was tamped into a glass tube. Through such a tube water was forced and platinum electrodes were inserted in the liquid on both sides of the diaphragm. As long as the water was being forced through the diaphragm an electric current manifested itself whose electromotive force was proportional to the hydrostatic pressure. The E.M.F. was independent of the structure and thickness of the diaphragm, which is in complete analogy with the phenomena involved in elec-

48 The German terms "Strömungsströme" for "hydrodynamic currents" and "Strömungspotentiale" for "hydrodynamic potentials" are perhaps more descriptive than the English equivalents. Transl.

${ }^{49}$ G. Quincke, Pogg. Ann. d. Physik. 107, 1 (1859); 110, 38 (1860). 
troendosmosis. The same results were obtained by Zöllner, ${ }^{50}$ Edlund, ${ }^{51}$ Haga, ${ }^{52}$ and Clark. ${ }^{53}$

The theory of hydrodynamic currents is similar to that of electroendosmosis, -it is the reverse of the latter. The movable ionic layer of the double layer is being sheared by the mechanical water current in the capillary. Hence the further end of the capillary becomes enriched in the ions of which this layer is made up. This does not occur, to be sure, to an analytically demonstrable extent, but sufficiently to give rise to a potential difference between the two ends of the tube. Figure 30 is an attempt to visualize this process. The liquid flows through the capillary $\mathrm{AA}^{\prime}$ into the larger vessel $\mathrm{BB}^{\prime}$. As long

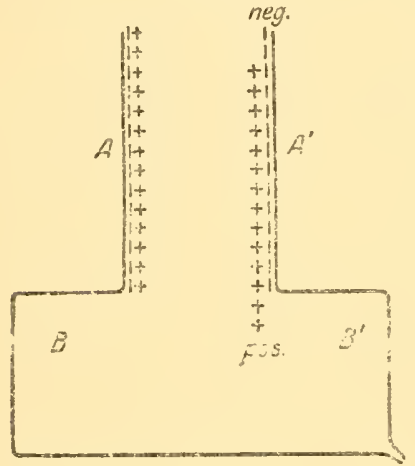

FIG. 30 as the liquid remained stationary a double layer was being formed in the manner indicated along the wall $\mathrm{A}$. When the liquid is set in motion, it tears along with it the movable layer adjacent to it, while the layer adhering to the wall remains so, as indicated along wall $\mathrm{A}^{\prime}$. In this way the upper end becomes negatively and the lower end positively charged.

The value $\mathrm{E}$ of the potential difference between the ends of the tube is according to Helmholtz, and including Pellat and Perrin correction for the dielectric constant (see page 249), as follows:

$$
\mathrm{E}=\frac{\zeta \cdot \mathrm{P} \cdot \mathrm{D}}{4 \pi \cdot \eta \lambda}
$$

(where $\eta$ is the viscosity of the fluid, $\lambda$ its specific electric conductance, $P$ the hydrostatic excess of pressure between the ends of the tube, $\mathrm{D}$ the dielectric constant, $\zeta$ the potential of the double layer on the capillary wall-or the "adsorption potential"). The E.M.F. of the current of flow is therefore directly proportional to the hydrostatic pressure and to the adsorption potential. It is inversely proportional to the viscosity of the liquid, for the more viscous the

${ }^{30}$ F. Züllner, Pogg. Ann. d. Physik. 148, 640 (1873).

${ }_{1}$ E. Edlund, Pogg. Ann. d. Physik. 1E6, 251 (1875); Wied. Ann. 1, 184 (1877).

62 H. Haga, Wied. Ann. 2, 326 (1877): E, 287 (1878).

63 J. W. Clark, Wied. Ann. 2, 335 (187\%). 
liquid the more slowly it moves at the same pressure and consequently the fewer ions it can tear along with it. The E.M.F. is also inversely proportional to the electric conductance, for the better conductor the liquid is the less of a potential difference is allowed by it to develop between the two ends of the tube. ${ }^{54}$ Furthermore, E is directly proportional to the dielectric constant, for the greater the value of the latter, the easier it is to separate the ions from each other. The proportionality fact or $\frac{1}{4 \pi}$ is introduced in order to reduce the value to electrostatic units. This equation can be used to calculate the potential $\zeta$ on the wall, after having measured the electromotive force $\mathrm{E}$ of the hydrodynamic current. The proof for the correctness of the absolute value of so calculated potentials suffers from the same difficulties as in the case of endosmosis. In this case, however, it depends only on the correctness of the factor $\frac{1}{4 \pi}$, otherwise the relative correctness of the equation may be accepted as having been demonstrated.

Thus the hydrodynamic current method represents a means for the study and calculation of adsorption potentials, and also for the study of the influence of the chemical character of the wall and of the liquid upon these potentials. These studies were made chiefly in glass capillaries, and glass should have behaved, in respect to its chemical properties, quite similarly to other silicates such as clay and kaolin. It is obviously of interest to find out whether the results obtained by the use of these methods agree with the results obtained by endosmosis and cataphoresis methods with clay and kaolin. Of the not very numerous studies in this field ${ }^{55}$ only two are particularly adequate in answering this question. These are the studies by Kruyt ${ }^{56}$ and by Freundlich and Rona. ${ }^{57}$ In general, it may be

${ }^{54}$ It is to be noted that in the equation for electroendosmosis (page 249) the conductivity did not enter directly. It entered only indirectly insofar as the conductance of the liquid in the pores of the diaphragm affected the fall of the potential per centimeter of the externally applied tension, or insofar as it affected the intensity of the electric field.

${ }_{55}$ A. T. Cameron and E. Oettinger, Phil. Mag. [6] 18, 586 (1909). A. Grumbacher, Ann. d. chim. et de phys. [S] 24, 433 (1911); L. Riéty, Ann. d. chim. et de phys. [8] 30, 1 (1913).

${ }^{56}$ H. R. Kruyt, Kolloid-Zeitschr. 22, 81 (1918).

${ }^{67}$ H. Freundlich and P. Rona, Sitzungsber. d. preuss. Akad. d. Wiss. 1920, p. 397 . 
stated that the theoretical expectations were to a great extent confirmed.

First it appeared that glass becomes in most cases negatively charged towards aqueous solution. By means of high hydrogen ion concentrations of the solution the current potential can be reduced to zero, but the sign of the charge could not be reversed in this way. Bivalent cations $\left(\mathrm{Ba}^{++}\right)$abolish the current potential in lower con-

TABLE 42

\begin{tabular}{|c|c|c|c|c|c|c|c|c|}
\hline \multirow{2}{*}{$\begin{array}{c}\mathrm{KCl} \\
\text { concentra- } \\
\text { tion }\end{array}$} & \multicolumn{3}{|c|}{ In millivolts } & \multirow{2}{*}{$\begin{array}{c}\mathrm{BaCl}_{2} \\
\text { concen- } \\
\text { tration }\end{array}$} & \multirow{2}{*}{$\mathrm{E}$} & \multirow{2}{*}{5} & \multirow{2}{*}{$\begin{array}{c}\mathrm{AlCl}_{3} \\
\text { concen- } \\
\text { tration }\end{array}$} & \multirow{2}{*}{$\mathbf{E}$} \\
\hline & & $\mathrm{E}$ & 5 & & & & & \\
\hline 0 & + & ca. 350 & - & 0 & + ca. 350 & - & 0 & t ca. 350 \\
\hline 50 & & 102 & 19.9 & 10 & 139 & 10 & 0.5 & $\begin{array}{l}+52 \\
+\quad 5\end{array}$ \\
\hline 100 & & 57 & 22.4 & 25 & 79 & 14.2 & 1 & $-\quad 42$ \\
\hline \multirow[t]{2}{*}{250} & & 23 & 23.0 & 50 & 44 & 16.0 & 2 & -122 \\
\hline & & & & 100 & 25 & 18.1 & 3 & 129 \\
\hline 500 & & 13 & 23.3 & 200 & 9 & 12.7 & 4 & 100 \\
\hline \multirow[t]{3}{*}{1000} & & 4.0 & 15.4 & 1000 & 1.1 & 7.6 & 10 & 52 \\
\hline & & & & 40000 & \multirow{2}{*}{\multicolumn{2}{|c|}{$\begin{array}{l}\text { No reversal of sign } \\
\text { of charge }\end{array}$}} & 100 & - \\
\hline & & & & & & & 500 & $-\quad 1.4$ \\
\hline \multicolumn{2}{|c|}{$\underset{\text { concentration }}{\mathrm{HCl}}$} & \multicolumn{2}{|l|}{$\mathrm{E}$} & 5 & $\begin{array}{l}\text { p-Chloraniline } \\
\text { chloride } \\
\text { concentration }\end{array}$ & \multicolumn{2}{|c|}{$\mathrm{E}$} & $\zeta$ \\
\hline \multicolumn{2}{|l|}{0} & \multicolumn{2}{|c|}{ + ca. 350} & - & 0 & \multicolumn{2}{|c|}{+ ca. 350} & - \\
\hline \multicolumn{2}{|l|}{25} & \multicolumn{2}{|c|}{77} & 22.1 & 31 & \multirow{2}{*}{\multicolumn{2}{|c|}{114}} & 13.9 \\
\hline 50 & & & 43 & 24.8 & & & & \multirow[b]{2}{*}{19.6} \\
\hline \multicolumn{2}{|l|}{$\begin{array}{r}50 \\
100\end{array}$} & & 22 & 25.4 & 62 & & 65 & \\
\hline \multicolumn{2}{|l|}{250} & & 7.7 & 22.1 & 124 & & 26 & 16.6 \\
\hline \multicolumn{2}{|l|}{500} & & 3.1 & 17.5 & 310 & & 12.2 & 14.5 \\
\hline \multirow{2}{*}{\multicolumn{2}{|c|}{$\begin{array}{r}1000 \\
50000\end{array}$}} & & 1.2 & 13.6 & 500 & & 4.9 & 11.8 \\
\hline & & \multicolumn{3}{|c|}{$\begin{array}{l}\text { No reversal of sign of } \\
\text { charge }\end{array}$} & 1000 & & 1.8 & 9.7 \\
\hline
\end{tabular}

centrations than univalent cations. Trivalent cations $\left(\mathrm{Al}^{+++}\right)$reverse the sign of the current potential even in the lowest concentrations, while at higher concentrations the potential is reduced to zero again. This was shown by the following experiment by Kruyt: Various liquids were forced through a glass capillary and yielded the values for hydrodynamic current potential shown in table 42 , at a water pressure of $1 \mathrm{~cm}$. of mercury. The concentrations are given in micromols $(1 \mu-\mathrm{mol}=0.000,001 \mathrm{~mol}) ; \mathrm{a}+$ sign before the value of 
$\mathrm{E}$ denotes that the lower end of the capillary is positive in respect to the upper end. The potential $\zeta$ of the wall of the capillary was calculated from the corrected Helmholtz equation.

It is of particular interest to observe that notable hydrodynamic potentials were obtained only in very dilute solutions of electrolyte. While in $10^{-5} \mathrm{~N} \mathrm{KCl}$ solutions of $20-40$ millivolts were obtained, no measurable potentials were observed in $10^{-3}$ to $10^{-2} \mathrm{~N}$ solutions.

We have already stated on various occasions that the presence of an excess of neutral salts diminishes potentials (diffusion-, interphase boundary-, membrane-potentials), and the same was the case in electroendosmosis experiments, where an excess of neutral salt caused a diminution in the amount of transported water. But in all of these cases an excess of salt of an entirely different order of magnitude was involved. Hydrodynamic potentials may, in general, be properly observed only in electrolyte solutions of concentrations of the order of magnitude of $10^{-6}$ to $10^{-4}$ normal. The cause for this is the fact that the current potential depends upon the conductivity of the solution. By increasing the concentration of the salt, in the first place the adsorption potential $\zeta$ is greatly diminished; secondly, the current potential $\mathrm{E}$ produced by a given adsorption potential and a given pressure is very profoundly depressed. It must be recalled that in the equation given above (page 280) the conductivity $\lambda$ is in the denominator of the fraction.

By calculating from the current potential $\mathrm{E}$ the potential of the wall $\zeta$ towards the solution, with the help of the corrected Helmholtz equation, the values given in the above table were obtained by Kruyt. It is notable that the values of the $\zeta$ potential do not vary as much with the electrolyte concentration as those of the $\mathrm{E}$ potential. This corresponds to the fact that the phenomena of endosmosis and cataphoresis, which show a greater parallelism with the $\zeta$ potential, do not depend to so great an extent upon the electrolyte concentration as hydrodynamic potentials do (since the specific conductivity does not appear in the equation for endosmosis).

It will be stated only in a corollary way that in the course of solid particles falling through a layer of water a potential difference develops between the upper and the lower ends of the water column. This is the potential of falling particles. The phenomenon is the reverse of the hydrodynamic potential, and the two are entirely analogous. 


\section{The influence of electrokinetic potentials upon the boundary surface tension}

For the sake of completeness we must mention here a mechanical manifestation of electrokinetic potentials, a more comprehensive discussion of which will be reserved for the chapter on colloids in a later volume of this work. According to the observations of Lippmann, in conjunction with the discovery of the capillary electrometer and with the Helmholtz theory relating to it, the double layer at a phase boundary exercises an influence upon the surface tension. Every individual stratum of the double layer, because it consists of similarly charged particles, tends to expand or to to extend itself, thus working in opposition to the ordinary surface tension. Consequently the surface tension at an interphase boundary must depend upon the potential difference. Now, when one of the phases is solid or' at least much more viscous than the other, this expansion can occur only in that ionic layer which is in the more fluid or less viscous phase. This tendency to expand on the part of the layer at the surface of the liquid is antagonistic to the ordinary surface tension. The total tension is therefore dependent upon the absolute value of the electric density of the double layer. It is at a maximum when this density $=0$; this is the case when the potential of the double layer is also $=0$, or hence, at the isoelectric point. The theory was extended by Bredig ${ }^{58}$ to colloidal solutions, and Michaelis ${ }^{59}$ showed, for colloidal solutions of protein substances, that, in fact, the isoelectric point (determined by cataphoresis experiments) is identical with the maximum of the surface tension (as determined by estimations of flocculation optima). This aspect will be further discussed in the following volume.

\section{A summary of electrokinetic phenomena}

In conclusion it may be useful to present a systematic summary of all electrokinetic phenomena. Let us imagine a solid wall and a fluid movable in reference to this wall. If an external potential is applied in a direction tangential to the wall, a displacement of the fluid along the wall will occur. When the wall is not mechanically displaceable (a capillary or a diaphragm), the fluid is displaced along

53 Bredig, Anorgan. Fermente. Leipzig 1901.

s9 Summarized by L. Michaelis, Nernst-Festschrift. Halle 1913. 
the wall: this is electroendodmosis. When the solid wall is displaceable (suspended particles) but the fluid is not displaceable (either because it is in an enclosed chamber, or, when in a U-tube, because hydrostatic differences in pressure can not be maintained), then the solid wall is moved along the fluid:- this is electrophoresis.

Conversely, when the solid wall is mechanically displaced along the fluid, a potential is formed in the direction of this displacement. If the wall is fixed in place (a capillary or a diaphragm), and the fluid flows along the wall, then a hydrodynamic current is formed. The fluid being stationary and the solid wall movable (falling particles), a potential of falling particles arises.

\section{The splitting of phase boundary potentials}

\section{The relation of phase boundary potentials to adsorption potentials}

In comparing the chapters on phase boundary potentials (pages $183 \mathrm{ff}$.) and adsorption potentials it appears rather remarkable that the potential difference occurring at an interface may be considered in two entirely distinct ways. Taking, for example, the phase boundary glass-water and considering the potential as a phase boundary potential, then the latter depends exclusively upon the $\left[\mathrm{H}^{+}\right]$in the solution. But if the potential is considered as an adsorption potential, then it depends upon all the ions, and to an especially great extent upon any trivalent cation present in the solution, which would be of no significance for the phase boundary potential. Thus the potential difference at the interface glass-aqueous solution, for example, does not appear to be rigidly defined, but may differ according to the nature of its manifestation which is taken into account. We regarded it as a phase boundary potential when it manifested itself in the form of an electromotive force in a Haber glasschain. We regard it as an adsorption potential when it manifests itself in the form of electrokinetic phenomena. This discord was for a long time a source of an ambiguity, which however is lately being slowly dispelled, through the explanations which were developed by Freundlich. For a better understanding of this problem we shall present the following considerations offered by Haber. ${ }^{60}$

Let us take the chain shown in figure 31. A silver electrode on the

60 F. Haber, Ann. d. Physik. [4], 927 (1918), cf. p. $949 . \quad$ R. Beutner, Dissert., Karlsruhe (1908). 
right is in contact with a solution of $\mathrm{AgNO}_{3}$ which is saturated with $\mathrm{AgCl}$. Into the same solution on the left side there is immersed another silver electrode, but not directly, being covered with a layer of fused and solidified $\mathrm{AgCl}$. This system is in a state of chemical equilibrium. When the two electrodes are connected by a metal wire there will be no flow of current. ${ }^{61}$ This system, therefore, contains three interfaces, a, b, and c. Each of these may be regarded as the source of a potential difference; $\mathrm{c}$ is the source of an ordinary metal electrode potential. In order to render this chain currentless the potential at $\mathrm{c}$ must be equal to that of $\mathrm{a}+\mathrm{b}$. Hence the introduction of the layer of solid $\mathrm{AgCl}$ has not altered the potential difference between the silver electrode and the solution. This can be summarized by the following generally applicable statement: When between two phases $A$ and $C$, which are in a state of chemical equilibrium,

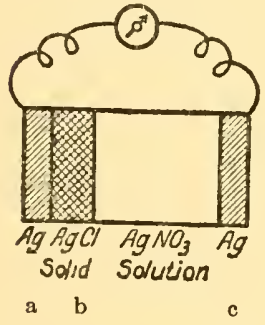

FIG. 31 a third phase $B$ is introduced, which is also in a state of equilibrium with the first two phases, the potential difference between $A$ and $B$ is not altered thereby. From this it follows that the total potential from $\mathrm{A}$ to $\mathrm{C}$ is decomposed into two stages through the introduction of the third phase.

Now if we employ a piece of silver covered with solid $\mathrm{AgCl}$ in one case as a pole of a galvanic chain, and in a second case in the form of a colloidal solution in a cataphoresis experiment, we shall find that in the first case the total potential of the silver is manifested towards the solution, while in the second case there is manifested only the potential difference between the adjacently moving surfaces, i.e., between the solution and the solid $\mathrm{AgCl}$. In this way it becomes clear why the superficially considered, identical interface (Ag-aqueous solution) will show a different electromotive potential $\epsilon$ depending upon other conditions than the electrokinetic potential $\zeta$. The cleanliness of the surface of the silver is immaterial for the electromotive potential, while for the electrokinetic potential it becomes, on the contrary, very noticeable when the silver becomes coated with the chloride or oxide depending

${ }^{61}$ Solid $\mathrm{AgCl}$ has sufficient conductance to permit of the measurement of the potential of this chain at least by means of electrostatic instruments. The conductance is an electrolytic and not a metallic one. 
upon the nature of the solution. Really pure metal surfaces do not last for any length of time in solutions, and most metals always become coated, at least with a layer of oxide.

It is furthermore clear that the layer interposed between $\mathrm{A}$ and $\mathrm{C}$ need not be a third phase. The interposition of a layer in which, because of interface effects, the ions would have a different distribution from that in the interior of the phase will suffice to resolve the potential between $\mathrm{A}$ and $\mathrm{C}$ into two stages. Since the composition of these interface layers changes but gradually in the direction of the interior of the fluid, it may be also assumed that the potential drop caused by such an adsorption layer will be altered in a gradual way represented by a smooth potential-gradient curve. ${ }^{62}$ It may even happen that this curve will show a maximum and minimum, and that a portion of this potential curve will have a sign different from that of the total potential. ${ }^{63}$ Thus Freundlich and Gyemant ${ }^{64}$ described cases in which they found on one and the same experimental material different signs before the values of $\epsilon$ and $\zeta$. In figure 32 is shown Smoluchowski's schematized graphic representation of the gradual fall of potential

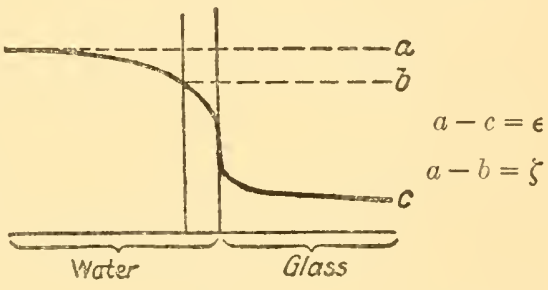

FIG. 32 at an interphase layer.

If this interpretation is correct, we should expect a discrepancy between the electromotive and the electrokinetic potentials in all those cases in which an adsorption layer is present at the interface, and this should always be the case with negligible exceptions.

In figure $32, \mathrm{~A}$ represents the interface of water-glass, and $\mathrm{AB}$ is the thickness of the adhering stationary water layer. The curve represents schematically the change of potential; in the interior of the water phase it is at the level c. The electromotive potential drop, which is manifested in the case of the glass chain, is $\epsilon=a-c$. The electrokinetic potential drop corresponds to $\zeta=\mathrm{a}-\mathrm{b}$.

${ }^{62}$ จ. Smoluchowski, in L. Graetz Handbuch der Elektrizität und des Magnetismus. 1912 and 1921. Bd. II.

${ }^{63}$ H. Freundlich, Kolloid-Zeitschr, 28, 240 (1921).

${ }^{64}$ Freundlich and Gyemant, Zeitschr. f. physikal. Chem. 100, 182 (1922). 
Although this problem is of great importance in colloidal chemistry and in biology, it does not appear desirable to dwell upon it at greater length, as long as the experimental material pertaining to it remains as scant as it is. The fundamentals involved are still uncertain, and yet the great importance of this problem, together with that promise of its early solution which has been given of late, especially by Freundlich's researches, induced the author to present the above provisional outline.

\section{Coehn's rule}

All of the considerations of electrokinetic phenomena in the preceding pages were made with the assumption that one of the two phases was water or an aqueous solution. We shall now briefly touch upon the available facts and theory concerning these phenomena, when a fluid other than water is the displaceable phase. The theoretic treatment of this case is all the more difficult, since too little is known about the dissociation of most solvents or of the electrolytes dissolved in them. An empirical rule has been worked out by Coehn. This investigator determined ${ }^{65}$ a large number of solid wall-potentials by means of electroosmotic experiments with glass or quartz capillaries, and also in electrophoresis experiments on the migration of liquid drops in an immiscible fluid. He first determined chiefly the sign of the charge, and later he also attempted to estimate quantitatively the potential by means of endosmotic measurements and from Helmholtz's equation. ${ }^{66}$ While Smoluchowski ${ }^{62}$ showed that these numerical values could not be utilized without further amplification, still the qualitiative results are at least partially acceptable. Coehn proposed the following rule: Every substance becomes negatively charged with respect to another substance of a higher dielectric constant, and it becomes positively charged with respect to substances of lower dielectric constants. When the solid wall is a glass capillary, then all substance whose dielectric constant (D.K.) $>5$ are positively charged, and those whose constant $<5$ negatively charged; see, for example, table 43 .

The same rule applies to the sign of the electric charge resulting from the friction of two solid substances. For it is generally ap-

Bs A. Coehn, Wiedem. Ann. 64, 217 (1898).

of A. Coehn and U. Raydt, Ann. d. Phys. 30, 777 (1909). 
parent that solid wall-potentials form the connecting link with the oldest known electric phenomenon, viz., friction electricity, which is still the most obscure of all electric phenomena.

It is still unknown whether in these cases the free electricity is represented by ions or by free electrons, although for substances in a non-metallic state we must consider the participation of free electrons as highly improbable. No contradiction can be found in Coehn rule to the laws governing the better known relationships. On the other hand to accept it as a universally applicable law would be far fetched. For it was shown, for instance, that the sign of the charge of water against glass is reversed by the presence of a trace of an aluminum

TABLE 43

\begin{tabular}{|c|c|c|}
\hline & Diel, constant & $\begin{array}{l}\text { Sign of charge } \\
\text { against glass }\end{array}$ \\
\hline 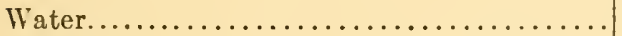 & 81 & + \\
\hline 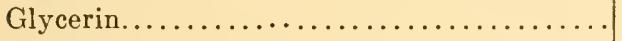 & 56 & + \\
\hline 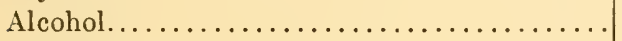 & 26 & + \\
\hline Acetic acid $\ldots \ldots \ldots \ldots \ldots \ldots \ldots \ldots \ldots$ & 9.7 & + \\
\hline Aniline.$\ldots \ldots \ldots \ldots \ldots \ldots \ldots \ldots \ldots$ & 7.2 & + \\
\hline Propionic acid...................... & 5.5 & $-(1)$ \\
\hline 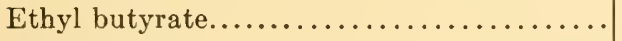 & 5.3 & + \\
\hline Amyl acetate...$\ldots \ldots \ldots \ldots \ldots \ldots \ldots$ & 5.2 & - \\
\hline 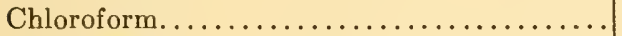 & 5.02 & - \\
\hline 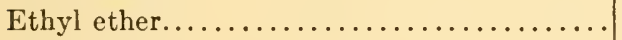 & 4.25 & - \\
\hline 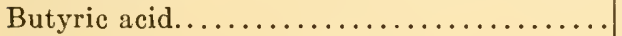 & 3.16 & - \\
\hline 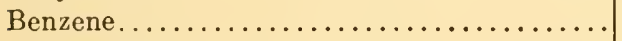 & 2.25 & - \\
\hline 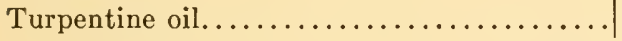 & 2.23 & - \\
\hline
\end{tabular}

salt, which could certainly have no detectable effect upon the dielectric constant of the water. There is also no occasion to speak of the superposition of the established ionic laws upon an universally valid Coehn's rule as of a fundamental principle. Undoubtedly all these phenomena may be explained in a coördinated way. Our present day theories extend only as far as our knowledge of the ionization of the phases permits. For all other cases Coehn's rule may be accepted as being empirically correct to a certain extent. Recently R. Keller ${ }^{67}$ made use of Coehn's rule for the explanation of biologically important processes, especially of histologic staining. It is possible

${ }^{67}$ R. Keller, Elektrohistologische Untersuchungen an Pflanzen und Tieren. Prag 1920. 
that he, in spite of considerable errors, has also brought forward some useful suggestions, which, however still await experimental verification.

The following interpretation of Coehn's rule appears to the author the most plausible. When one of the phases is of a distinctly ionogenic character (silicic acid, ferric oxide), then Coehn's rule does not apply at all; the laws covering this case were stated before. For here the dielectric constant plays a subordinate part, and, according to the nature and the concentration of the dissolved electrolytes, the water may be either positively or negatively charged with respect to the other phase. When the non-aqueous phase possesses a great adsorption capacity (charcoal), Coehn's rule does not hold again; for the charge on charcoal may be either positive or negative, again depending upon the electrolytes in the contiguous aqueous phase. On the other hand, the rule does seem to be valid in those cases where at least one of the substances involved is of a chemically indifferent nature, and where, at the same time, no great adsorption capacity, such as that of charcoal, is present, and where, therefore, the adsorption of ions at the interphase boundary is of the "apparent" type (see page 234). In these latter cases the following might be a general interpretation of Coehn's rule, which is offered with reservations only as a provisional working hypothesis. $T$ ? cause of the charge on the interface lies chiefly in the traces of watu posing water the OH-ion is the more si. active one. Since water ionizes to a greater extent in a medium possessing the higher dielectric constant, there occurs at the surface of such a medium an accumulation of $\mathrm{OH}$-ions, and hence this surface appears to be negatively charged with respect to its own medium.

\section{The significance of adsorption potentials in physiology and colloidal chemistry}

The first question which occurs to us is whether adsorption potentials bear any relation to the electric potentials and currents which we observe with the help of electrical measuring instruments in the living organism. This question had to be apparently answered in the negative. For it was shown above that the adsorption potentials manifest themselves only through electrokinetic phenomena (electroendosmosis, etc.) and that they can not be led off by means of metallic electrodes. As soon as we attempt to lead off any potential 
present on a wall to a measuring instrument we always obtain only the phase boundary potential, and not the adsorption potential, and these two, as was previously shown, are not identical. The only case in which an adsorption potential yields a direct electric manifestation which is detectable by electric measuring instruments is that of the hydrodynamic potential (or the potential of falling particles). But as we have shown before, the conditions prevailing in the living organism are most unfavorable for the generation of hydrodynanic potentials.

But, on the other hand, the adsorption potential manifestations that are undetectable by electric measuring instruments are quite clearly present in colloidal solutions. Only adsorption potentials can exercice an influence upon the state of a colloidal solution. For this state is determined by the tensions existing at the boundary surfaces of the phases. It is on these tensions that changes in the form and size of the particles, coagulation and peptization depend. The most important single agent affecting these tensions is the electric charge. But since all changes in the colloidal state depend upon the reciprocal action of adjacent boundary sulfaces, therefore only those double layers can be of significance which are located on the boundary surfaces, on the walls of the dispersed particles and in the adjacent to them layers of the $d^{*}$ rsion medium. This introduces us to another mode of ar $\eta$. of adsorption potentials other than their electrokinetic manisisiafions, which, moreover, prepares us for the theory of colloidal behavior.

But all of these processes are really of much greater importance for the interpretation of other significant physiological problems than would first appear from the above discussion. If we consider the capillary-electrical phenomena in diaphragms with very narrow pores, such as can be observed in collodion or gelatin membranes, especially the polarization phenomena described by Bethe, then the physiological applicability of these processes to such problems as currents of rest, action currents, and of the stimulation of muscles and nerves becomes so evidently manifold that we can hardly attempt to discuss them at greater length in this volume. Bethe ${ }^{i 8}$ had made an important beginning in this direction in which the exceptional position of the H-ions is again emphasized. There also the question narrows itself down to the alternative as to whether to base the

${ }^{88}$ A. Bethe, Pflügers Arch. f. d. ges. Physiol. 163, 147 (1916). 
interpretation of bioelectric currents, ionic permeability, stimulation, and other equally important biological processes upon the activity of forces at interfaces between an aqueous and a non-aqueous phase (page 191) or upon the capillary electric phenomena in narrow-pored gelatinous diaphragms.

The limits of the designated subject of this book were somewhat exceeded in the last chapters, though it was not attempted to present a complete description of the bordering problems. However, it is just in this impossibility of sharp delimitation of the subject and in its constantly growing sphere of influence in other fields that its fundamental importance is manifested. Nor does the future seem far distant when the small portion of physical chemistry, here presented, doubtlessly improved and changed in many respects, will become the ultimate basis of that rational physiology which we expect to see developed in the next few decades. 


\section{AUTHOR INDEX}

Abbott, 30

Adams, 67

Armstrong, 247

Arrhenius, 7, 25, 42, 113, 115

Barendrecht, 167

Bartell, 243

Bechhold, 80, 82

Becquerel, 247

Bernstein, 210

Bethe, 259, 272 ff., 291

Beutner, 149ff., 184ff., 211ff., 285

Biilmann, 173

Bjerrum, 67, 69, 87, 90, 101, 104, 114, 116, 120ff., 126, 180, 181

Boden, 80, 82

Bodländer, 31

Bolin, 54

Boltzmann, 119

Bray, 30

Bredig, 60, 284

Brighton, 27

Brinkman, 85

Brüning, 215

Burton, 253

Cameron, 281

Chanoz, 179

Clark, W. M., 102, 173

Clark, J. W., 280

Coehn, 288ff.

Cormack, 31

Coulter, 269

Cremer, 149, 197-8, 208, 211

Cumming, 179, 180

Daniell, 247

Davidsohn, 66, 70

Debye, 126

Dernby, 73
Donnan, $220 \mathrm{ff}$.

Drucker, 31, 111

DuBois Reymond, 209, 247

Eckweyller, 66

Edlund, 280

Ellis, 251

Euler, 31, 54, 72, 73

Fajans, 125

Fales, 180

Falk, 66

Faraday, 247

Fels, 43

Fischer, E., 35

Freundlich, 199, 238, 241, 277-8, 281, $285,287-8$

Garmendia, 30

Ghosh, 114, 117-8

Giblos, 233, 241

Gilchrist, 180

Gillespie, 104

Glixelli, 259

Grineff, 260

Grotthus, 149

Grumbacher, 281

Gudzent, 80, 82

Gyemant, 104, 241, 259-61, 268, 287

György, 137

Haber, 149, 167, 184-5, 198, 203, 211, 278,285

Haga, 280

Hamburger, 229

Hantzsch, 37, 97

Hardy, 64

Hartleben, 236

Heesch, 268

Heidenhain, E., 247 
Helmholtz, 209, 247, 248ff., 271

Henderson, L. J., 43

Henderson, P., 178-9

Hermann, 209

Hertz, 117

Heydweyller, 10, 25

His, 80, 81

Hittorf, 272

Höber, 210-11, 269

Hü.ckel, 126

Jahn, 114

Jürgensen, 247

Kallmann, 117

Kanitz, 60, 72, 80, 82

Keller, 20, 288

Klemensiewicz, 149, 184, 198, 211

Kohler, 80, 82

Kohlrausch, 10, 25, 177

Koppel, 104, 108

Krüger, R., 129

Krüger, A., 46

Kruyt, 281-2

Lachs, 235

Lamb, 180, 250

Larson, 180

Lewis, G. N., 27, 126, 180

Lichtwitz, 80

Linderstrøm-Lang, 173

Lippmann, 284

Loeb, J., 149, 185, 228, 260, 268

Löffler, 242-3

Löwenhertz, 19, 26

Lundén, 25, 31, 60, 72, 93

Luther, 31

MacInnes, 123

Madsen, 31

Masius, 238

Meyer, G., 198

Meyer, H., 229

Michaelis, 27, 30, 31, 36, 43, 46, 49, 56, $60,64,66,67,69,70,73,104,129$, $137,147,185,189,235,238,242,244-5$, $260,269,277,284$
Miller, 243

Milner, 114, 119-20

Mizutani, 67

Moers, 9

Nernst, 7, 9, 12, 14, 26, 103, 113, 125, 156ff., 175, 184-5, 211, 218, 255, 277

Noyes, A. A., 31, 123

Noyes, H. M., 66

Oettinger, 281

Ogawa, 243

Oker-Blom, 210

Ostwald, Wilh., 26, 30, 31, 91, 96, 210

Overton, 229

Paul, 80, 81

Pellat, 249

Perrin, 248-9, 255ff., 261, 264, 266

Pfeffer, 215, 229

Planck, $177-80$

Porret, 247

Procter, 228

Putter, 269

Quincke, 247, 279

Randall, 126

Raydt, 288

Reimer, 209

Reuss, 246

Reynolds, 252

Riesenfeld, 125, 184, 211, 218

Riéty, 281

Ringer, 80

Rona, 31, 36, 43, 73, 82, 85, 137, 199, $235,238,242,244-5,281$

Rothmund, 31

Rudolphi, 113

Rupert, 180

Sackur, 124

Salesski, 43

Schade, 80,82

Sebastian, 27

Smoluchowski, 250ff., $287 \mathrm{ff}$. 
Sørensen, 22, 27, 28, 43, 60, 74, 96, 103, 173

Spiro, 104, 108, 242-3

Strohecker, 34

Takahashi, 82, 85, 269

Thiel, 34

Thomson, J. J., 14

Tollens, 35

Toropoff, 259, 272

Traube, 215

van Dam, 85

Van Slyke, 109 van't Hoff, 7, 113, 114

Vosburgh, 180

Walden, 14, 31, 149-50

Walker, 31, 60, 72

Warburg, E., 131, 198

Weyl, 180

Wiedemann, 247

Wijs, 25

Wilson, J. A., 228

Winkelblech, 60, 72, 73

Wood, 72

Ziegler, 80, 82

Zöllner, 280 



\section{SUBJECT INDEX}

Acid, definition, 11, 16, 18

Acid-amides, 14

Acid-fuchsin, 37

Acidity, 94-109; determination, Chapter II; titratable, 95

Acidoid, 240, 261

Acids, apparent dissociation of, 32 ; degree of dissociation of, 48 ; dissociation of, 29-37; dissociation constants, tables, 31, 59; mixture of two - 46-48; polybasic, dissociation of, 55-60; pseudo-, 32, 118; pure, $\mathrm{pH}$ in solutions of, 37 ; solubility, 77,82 .

Active masses, 6, 160

Activity, 110ff., 132-3; coefficient, 121ff.; factor, 114, 160 ; of $\mathrm{H}$-ions, 128 ; theory, 87, 110ff., 126, 127, $134-5,160$

Adsorbents other than charcoal, 243

Adsorption, Chapter $\mathrm{X}$; apparent, 234, 268; definition, 231-2; equivalent -, of $\mathrm{H}$ - and $\mathrm{OH}$-ions, 242; by exchange of ions, 244; of electrolytes, 234, of $\mathrm{H}$ - and $\mathrm{OH}$-ions, 236,242 ; of other ions, 265 ; of ions and charge formation, 245

Adsorption potentials, Chapter $\mathrm{X}$; history of theory of, 277 ; relation to phase boundary potentials, 285 ; significance in physiology, 290

Affinity constants, 5, 30

Alcohol, 19

Alkalinity, determination of, Chapter II

Ammonia, 16

Ammonium bases, 16

Ampholytes, 17; definition, 18; dissociation of, 60ff., 141; isoelectric point, $65-7$; solubility, 86 ; table of dissociation constants and isoelectric points, 72-3

Ampholytoid, 240

Amphoteric electrolytes, see Ampholytes

Atomic model, 8, 9, 15

Autopotential, 117

Bases, definition, 11, 16, 18; dissociation of, 37-39; table of dissociation constants, 38

Basoid, 240

Blood (plasma), buffers in, 127; calcium ions in, 85; solubility of calcium carbonate in, 84 ; uric acid in, 80

Buffers, 43-49; 104-9; activity and $\mathrm{pH}$ of, $127-9$; in blood, 127 ; in seawater, 127 ; in urine, 128

Calcium ions in blood, 85

Calcium carbonate in blood, 84

Carbonic acid, dissociation constant of, 34-5

Cataphoresis, see Electrophoresis

Charcoal, adsorption of electrolytes, 234; adsorption of $\mathrm{H}$ - and $\mathrm{OH}$-ions, 236 ; as an ampholyte, 239, 243; neutralizing effect of, 242

Coehn's rule, 241, 288-90

Colloids, 265, 290

Concentration chains, 102, 160

Concentration effect, 196

Conductivity, coefficient, $121 \mathrm{ff}$; factor, 114; of H- and OH-ions, 152; in oil phases, 150; influence of ionic charge on, 117; molar, 112; of water, 24

Crystallization optimum, 70 
Current of falling particles, 279

Currents, hydrodynamic, 279

Degree of dissociation, 9, 14-15; of acids, 48-54; definition, 48

Diaphragms, as acidoids, 261; isoelectric point of, 259 ; potential on, 259 ; sign of charge of, 261,269

Dielectric constants, 13-15, 28s; of water, 14 ; tables of, 14,289

Diffusion potentials, Chapter VII; abolition of, 180

Dilution, effect of, on $\mathrm{pH}$ of buffers, 45

Dissociation, electrolytic, laws of, Chapter I; in non-aqueous solutions, Chapter V

Dissociation of: acids, 29-37; ampholytes, 60-69; bases, 37-9; polybasic acids, 55-60; strong electrolytes, Chapter III; water, 9-13, 19-22

Dissociation constants, 5 ; determination of, $91-3$; reduced, 129, 145; of water, 19-22, 24-28

Dissociation constants, tables of: acids, 31; ampholytes, 72-3; bases, 38; polybasic acids, 59; of water, effect of temperature on, 26

Dissociation residue, definition, 49; of acids, 48-54; 139 ; of ampholytes, 61, 141-3

Distribution coefficient, 186

Double layer, electric, 247,280

Electric double layer, 247,280

Electrode, gas, 164; quinhydrone, 171; reversible, 163

Electrode potentials, Chapter VI

Electroendosmose, 246, 254, 284; changes of $\left[\mathrm{H}^{+}\right]$in, 271; Helmholtz's theory, 248; Perrin's experiments, 258

Electrokinetic phenomena, Chapter X; summary of, 284

Electrolytes, strong, dissociation of, Chapter III; effect of $\mathrm{pH}, 131$

Electrolytic dissociation, Chapter I
Electromotive series of ions, 167-9; table, 168; of oils, 207

Electrons, 8

Electrophoresis, 250, 254, 284

Electrostriction, 113

Equivalent capacity of acids, 96

Exchange of ions in adsorption, 244

Falling particles, current and potentials of, 279,284

Formamide, 14

Freezing point depression, 119-123

Gas chains, theory of, 164-7

Gas electrode, 164

Gibbs principle, 241

Glass chain, 197

Glucose, 35-6

Glycerin, 14

Glycocoll, 17

Henderson's formula for diffusion potentials, 179

Hydrocyanic acid, 14

Hydrodynamic currents and potentials, 279, 285

Hydrogen atom, 9

Hydrogen ion, negative, 9

Hydrogen ion concentration, 21; determination of, 102-4; in acids, $39,46,77,82$; in ampholytes, 74,86

Hydrogen ion exponent, 22-4

Hydrogen number, 21

Hydrolysis of salts, $87-91$; table, 90

Indicators, 36-7; theory of, 96-102

Ions, migration velocity of, 174-6; table, 175; series of, $205,213,235$, 275

Isohydric solutions, 42

Isoelectric point of ampholytes, 65$73,76-7,91-3,143-5$; determination of, 69-73, 91-3; table of, 72-3

Isoelectric point of diaphragms, 259261 
Liquid junctions, see Diffusion potentials

Lithium hydride, 9

Mass action law, 3-7; deviations from, $110-7$

Membrane potentials, Chapter IX; relation of to phase boundary potentials, 228-31

Migration velocity of ions, 174-6; table, 175

Milner effect, 120

Moderators, 108

Neutral salts, definition, 87

Neutralizing capacity, 96

Nernst's formula for concentration potentials, 103, 161; evaluation of, 161

Nitriles, 14

Oil chains, 192

Oil phases, dissociation in, 150

Ostwald's law, 91

Osmotic coefficient, $121 \mathrm{ff}$.

Osmotic factor, 114

Osmotic pressure of ions, 159

Oxidation-reduction potentials, 169 73

Permeability, 210

$\mathrm{pH}$ scale, 22-4

Phase boundary chains, 191-5

Phase boundary potentials, Chapter VIII; and adsorption potentials, 285 ; calculation of, 183; origin of, 183; physiological applications of, 209; splitting of, 285

Phenolphthalein, 36-7

Phosphates, activity of, 130 ; buffers, 46

Planck's formula for diffusion potentials, 177

Polarization at phase boundaries, 218
Potentials, adsorption, Chapter X; diffusion, Chapter VII; electrode, Chapter VI; of falling particles, 279-83; hydrodynamic, 279-83; membrane, Chapter IX; oxidationreduction, 169-73; phase boundary, Chapter VIII; at precipitation membranes, 215

Pseudo-acids, 32, 118

Precipitation membranes, 215

Quinhydrone electrode, 177

Reaction, definition, 21

Reduced constants of acids, 129-31, 145-7

Regulators, see Buffers

Reversible electrodes, 163

Saloid, 240

Salts, definition, 11, 18

Salt-formation, Chapter IV

Saturation, definition, 78

Solubility, of acids, 77-86; of ampholytes, 86-7; of Ca-carbonate, 82; of uric acid, 80 ; partial and total, 78

Surface tension at interfaces, 284

Two acids, problem of, 46-8

Titratable acidity, 95

Titration capacity, 96

Titration curves, 99

Transport number, 218

Uric acid, 80

Water, dielectric constant, 14; dissociation of, 9-28; dissociation constant of, 12-3, 24-8; ions of, 13 ; properties of, 13-9

"Yielding power," 107

"Zwitter-Ion," 73 



\section{Sans Tache}

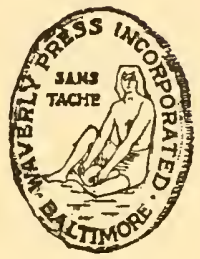




\section{Sans Tache}

T N THE "elder days of art" each artist or craftsman enjoyed the privilege of independent creation. He carried through a process of manufacture from beginning to end. The scribe of the days before the printing press was such a craftsman. So was the printer in the days before the machine process. He stood or fell, as a craftsman, by the merit or demerit of his finished product.

Modern machine production has added much to the worker's productivity and to his material welfare; but it has deprived him of the old creative distinctiveness. His work is merged in the work of the team, and lost sight of as something representing him and his personality.

Many hands and minds contribute to the manufacture of a book, in this day of specialization. There are seven distinct major processes in the making of a book: The type must first be set; by the monotype method, there are two processes, the "keyboarding" of the MS and the casting of the type from the perforated paper rolls thus produced. Formulas and other intricate work must be hand-set: then the whole brought together ("composed") in its true order, made into pages and forms. The results must be checked by proof reading at each stage. Then comes the "make-ready" and press-run and finally the binding into volumes.

All of these processes, except that of binding into eloth or leather covers, are carried on under our roof.

The motto of the Waverly Press is Sans Tache. Our ideal is to manufacture books "without blemish"-worthy books, worthily printed, with worthy typography - books to which we shall be proud to attach our imprint, made by craftsmen who are willing to accept open responsibility for their work, and who are entitled to credit for creditable performance. 
The printing craftsman of today is quite as much a craftsman as his predecessor. There is quite as much discrimination between poor work and good. We are of the opinion that the individuality of the worker should not be wholly lost. The members of our staff who have contributed their skill of hand and brain to this volume are:

Composing: John Crabill, Ray Kauffman, Herbert Leitch, Andrew Rassa, Nathan Miller, Arthur Baker, Charles Wyatt, Richard King, Edward Rice, George Moss, Walter Phillips, Henry Shea, Theodore Nilson.

Keyboard: Eleanor Luecke, Anna Rustic, Hannah Scott, Katharine Kocent, Minnie Foard, Vera Taylor, Helen Twardowicz.

Proof Room: Sarah Katzin, Mary Reed, Alice Reuter, Ruth Treischman, Ethel Strasinger, Lucille Bull, Angeline Eifert, Audrey Tanner, Dorothy Strasinger, Lillian Gilland, Geraldine Browne, George Southworth, Ida Zimmerman.

Casters: Kenneth Brown, Mahlon Robinson, Ernest Wann, Charles Aher, George Smith, Henry Loe, Frank Malanosky, Martin Griffen.

Press: Raymond Gallagher, Robert Gallagher, August Hildebrand.

Folder: Laurence Krug.

Culter: Howard Brown. 


\section{New and Useful Books in Related Fields}

The Effects of Ions in Colloidal Systems $\$ 2.50$

A study of an important problem by a world authority, Dr. Leonor Michaelis. Treats Adsorption of Ions; Electrical Double Layers; Properties of Charcoal; Charge, Adsorption and Flocculation; Donnan Equilibrium; Lyotropic Effect of Ions; Mixtures of Electrolytes.

\section{The Determination of Hydrogen Ions \$5.00}

A treatise on the hydrogen electrode, indicator, and supplementary methods of determining hydrogen ion concentrations, with an indexed bibliography of two thousand references on applications. An important contribution. Second edition. With an indicator chart in colors inserted. By W. Mansfield Clark.

\section{Hydrogen Ion Concentration of the Blood} in Health and Disease

A summary of the most important facts presented in concise form for the use of clinicians and workers in clinical laboratories. By J. Harold Austin and Glenn E. Cullen.

\section{THE WILLIAMS \& WILKINS COMPANY}

Publishers of Scientific Books and Periodicals

BALTIMORE, U.S. A. 





